\title{
5th EFLM Conference on Preanalytical Phase Zagreb (HR), 22-23 March 2019
}

\author{
Organizing Committee \\ Ana-Maria Simundic, Chair (HR) \\ Organizing Committee, members \\ Lora Dukic (HR) \\ Vanja Radisic Biljak (HR) \\ Silvia Cattaneo (IT) \\ Ana Dojder (HR) \\ Dora Vuljanic (HR)

\section{Scientific Committee} \\ Ana-Maria Simundic, Chair (HR) \\ Janne Cadamuro (AT) \\ Michael Cornes (UK) \\ Barbare De La Salle (UK) \\ Pinar Eker (TR) \\ Kjell Grankvist (SW) \\ João Tiago Guimarães (PT) \\ Mercedes Ibarz (SP) \\ Svetlana Kovalevskaya (RU) \\ Gunn B.B. Kristensen (NO) \\ Giuseppe Lippi (IT) \\ Mads Nybo (DK) \\ Zorica Sumarac (SR) \\ Edmée Van Dongen-Lases (NL) \\ Pieter Vermeersch (BE) \\ Alexander von Meyer (DE)
}




\title{
Preanalytical cases
}

\section{A PARADIGMATIC CASE OF SPURIOUS HYPERKALEMIA IN BLOOD GAS ANALYSIS}

\author{
G.L. Salvagno $^{1}$, D. Demonte ${ }^{1}$, G. Lippi ${ }^{1}$ \\ ${ }^{1}$ Section of Clinical Biochemistry, University Hospital of Verona, Verona, Italy
}

\begin{abstract}
BACKGROUND-AIM: We describe a paradigmatic case of spurious hyperkalemia in blood gas analysis due to inappropriate transport conditions of a heparinized syringe.

METHODS: A 51-year old male patient was admitted to the local hospital with dyspnea. Venous blood was drawn by a nurse into a $3.0 \mathrm{~mL}$ heparinized syringe (Smiths Medical), and sent to the central laboratory, for analysis of blood gases.

RESULTS: Blood gas analysis, performed with GEM Premier 4000 (Instrumentation Laboratory), revealed a substantially normal blood gas profile except a high value of potassium (i.e., $5.2 \mathrm{mmol} / \mathrm{L}$; local reference range on whole blood, 3.5-4.5 mmol/L). This result was unexpected by the requesting clinician, so that a second venous blood sample was immediately drawn by direct venipuncture into a $3.5 \mathrm{~mL}$ lithium-heparin blood tube (Vacutest, Kima), and sent to the laboratory for repeating potassium testing. The analysis, performed on Cobas 8000 (Roche Diagnostics) revealed normal values of plasma potassium (i.e., $4.6 \mathrm{mmol} / \mathrm{L}$; local reference range in plasma, 3.5-5.0 $\mathrm{mmol} / \mathrm{L}$ ) and hemolysis index (i.e., 5; $0.05 \mathrm{~g} / \mathrm{L}$ ). Due to suspect of spurious hemolysis, the heparinized blood was transferred from the syringe into a plastic tube and centrifuged. Both potassium and hemolysis index were then measured in this heparinized plasma, using Cobas 8000. The visual analysis of plasma after centrifugation was already consistent with the presence of hemolysis, which was then confirmed by high hemolysis index (i.e., 50; $0.5 \mathrm{~g} / \mathrm{L}$ ). The potassium value in centrifuged heparinized plasma was also consistent with hyperkalemia (i.e., $5.5 \mathrm{mmol} / \mathrm{L}$ ). Investigation of the case revealed that spurious hemolysis was attributable to syringe transportation to the laboratory in direct contract with ice for $\sim 15$ min. CONCLUSIONS: This case emphasizes the importance of fulfilling strict criteria for blood sample transportation and the urgent need of equipping point of care analyzers with interference indices.
\end{abstract}

Key Words: spurious hyperkalemia, blood gas analysis, preanalytical phase

\section{PREANALYTICAL CASE REPORT - UNEXPECTED CHANGE OF PATIENT IDENTIFICATION IN LIS}

\author{
$\underline{\text { E. Fliser }}^{1}$, B. Jevšnikar ${ }^{1}$, M. Gorenjak ${ }^{1}$ \\ ${ }^{1}$ Department for Laboratory Diagnostics, University Clinical Centre Maribor, Slovenia
}

BACKGROUND-AIM: We present a case report of unexpected change of patient identification in the laboratory information system (LIS) after activating two different sample requests for the same patient.

METHODS: Our laboratory received a sample request for a female patient (PATIENT X, with X presenting her family name) from the emergency department. The request is usually done with the help of patient's health insurance card with a unique number to prevent errors in identification. The request was done in the hospital information system (HIS) and transmited to LIS.

Later the laboratory received a request from an internal hospital department for additional tests for PATIENT Y, whereby the sample should be already in the laboratory. Again the request was created in HIS and activated in LIS, but the sample could not be found. According to both departments, PATIENT Y was moved from the emergency department and PATIENT X was the only person who was moved to the internal department in that time period. LIS had no longer the identification of PATIENT X. Instead of it, the identification of PATIENT Y was placed. In summary, the laboratory found the sample of PATIENT X with changed identification to PATIENT Y in LIS.

RESULTS: The emergency department did not provide the information how the first request was created. The request was probably made without the health insurance card, by asking the patient and indicating her girl's family name. After the patient was moved to the hospital department, the second request was made with the insurance card, whereby the system detected the right family name. When the request was activated in LIS, the first identification of the patient was overwritten.

CONCLUSIONS: The computer company was asked to prepare a new software version of LIS. In case of patient identification change, a warning is given now; the system asks whether to use the new identification from the given time on or to overwrite the patient's data.

Key Words: sample request, LIS, patient identification change

\section{PREANALYTICAL CASES IN CEREBROSPINAL FLUID ANALYSIS}

\author{
$\underline{\text { P. Broz }}^{1}$, J. Zenkova ${ }^{1}$, D. Rajdl ${ }^{1}$, J. Racek ${ }^{1}$, V. Petrikova ${ }^{1}$ \\ ${ }^{1}$ Department of Clinical Biochemistry and Hematology, University hospital in Pilsen, Charles University in Prague, Czech Republic
}

BACKGROUND-AIM: Poster presents two preanalytical cases involved in cerebrospinal fluid (csf) analysis.

METHODS: First case presents preanalytical error during collection of csf. Second case presents wrong indication of csf spectrophotometry. 
RESULTS: Case 1 - First patient was hospitalized at Hemato-oncology department. Lumbar puncture was repeatedly performed. In first and second sample only small changes were found. Third csf analysis revealed no mononuclear and no polynuclear cells. Glucose, lactate and total protein concentrations were bellow limit of detection. Additional analysis revealed low beta trace protein concentration $(0.42 \mathrm{mg} / \mathrm{L}) \mathrm{and}$ concentrations of natrium, potassium and chlorides with results of 144, $<0.4$ and $118 \mathrm{mmol} / \mathrm{L}, \mathrm{resp}$. During communication with department usage of local anesthesia was discussed. Analysis of solution with anesthetic revealed very similar concentration of natrium, potassium and chlorides. Case 2 - 38 years old female with subfebrilia and mild headache was admitted to the Department of Infectious diseases. Csf analysis was performed with results: total protein $1.2 \mathrm{~g} / \mathrm{L}$, glucose $3.4 \mathrm{mmol} / \mathrm{L}$, lactate $1.9 \mathrm{mmol} / \mathrm{L}$, polynuclear cells $2 / \mu \mathrm{L}$, mononuclear cells $50 / \mu \mathrm{L}$. There was no intention of presence of subarachnoid hemorrhage, computed tomography was not performed before csf analysis, but csf spectrophotometry was performed. Presence of oxyhaemoglobin was found. Magnetic resonance with angiography found internal carotid artery aneurysm. Patient was admitted to the Department of neurosurgery and coiling of aneurysm was performed.

CONCLUSIONS: First case presents the possibility of influence of local anesthetics usage on spinal tap. However, influence of solutions with local anesthetics does not have to be evident in all cases. Second patient presents interesting case, when csf spectrophotometry was not performed according to recommendations, however with no harm on patient's health, or maybe with positive influence on patient during diagnostic work up.

Key Words: cerebrospinal fluid, preanalytical phase, spectrophotometry

\title{
ROTATORIA WORM AS AN EXOGENOUS CONTAMINANT OF URINE SAMPLE
}

\author{
L. Gobec $^{2}$, E. Šoster Križnik ${ }^{3}$, E. Božnar Alič ${ }^{1}$, K. Pritržnik ${ }^{2}$ \\ ${ }^{1}$ Clinical Institute for Clinical Chemistry and Clinical Biochemistry, University Clinical Centre Ljubljana, Slovenia \\ ${ }^{2}$ Departement for Laboratory Medicine, General Hospital Celje, Slovenia \\ ${ }^{3}$ Departement for Pediatrics, General Hospital Celje, Slovenia
}

BACKGROUND-AIM: Several elements from environmental sources may contaminate the urine. Usually they come from the air, while urine is handled in the laboratory. These contaminants include pollen graules, plant cells and fungal spores. Other contaminats are very rare in our clinical practice and their identification is difficult.

METHODS: 4 years old girl was admitted to the alergological office because of recidival acute inhalatory infections and family anamnesis of asthma. Basic laboratory test were performed. Urinary sediment was prepared and evaluated using standard laboratory procedure with light microscopy.

RESULTS: During routine urine examination unknown worm was found in urinary sediment. Web search and video consultaltion with biologist revealed identification of Rotatoria organism. Rotatoria was present in urinary sample as an exogenous contaminant. Patient's mother told, that girl was playing a lot in the ponds around the family house. During scraping the worm was transfered from hands of the girl to the skin surrounding the genitalia, and from there it was washed out in the urine sample. Control urine sample, strikly adhering to the protocol for sample collection, was taken a week after. Result of urinary sediment didn’t reveal a presence of Rotatoria again.

CONCLUSIONS: Rotatoria is a class of worms of the subphylum Nemathelminthes. There are approximately 2000 known species which live wherever there is water. According to literature organism doesn't harm human health. Preanalytical phase is extremely important to avoid contamination of samples. Collaboration between different laboratory specialist is precious and valuable to solve such identification problems.

Key Words: preanalytical case, urine contamination, urinary sediment

\section{FLUSHING OF AN INTRAVENOUS CATHETER: A CAUSE FOR UNRELIABLE LABORATORY RESULTS}

\author{
$\underline{\text { R.C.C. Hengeveld }}^{1}$, M.C. Gerards ${ }^{3}$, B.E. Olofsen ${ }^{2}$, M.L. Ridderikhof ${ }^{2}$, V.F.H.A. Hakkenberg Van Gaasbeek ${ }^{2}$, P.A. Leenhouts ${ }^{2}$, E.C. Van Dongen- \\ Lases $^{1}$ \\ ${ }^{1}$ Amsterdam UMC, University of Amsterdam, Department of Clinical Chemistry, Amsterdam, Netherlands \\ ${ }^{2}$ Amsterdam UMC, University of Amsterdam, Department of Emergency Medicine, Amsterdam, Netherlands \\ ${ }^{3}$ Amsterdam UMC, University of Amsterdam, Department of Internal Medicine, Amsterdam, Netherlands
}

BACKGROUND-AIM: Phlebotomy is an error-prone process in which mistakes are difficult to reveal. This case report describes the effect on laboratory results originating from a blood sample collected in close proximity to an intravenous catheter.

METHODS: A 69-year-old male patient was referred to the Emergency Department where pneumonia was suspected. Phlebotomy was performed to collect blood samples to assess electrolytes, renal function, liver function, infection and hematological parameters.

RESULTS: The laboratory analysis showed reduced potassium and calcium concentrations. To prevent life-threatening cardiac failure the clinician decided to correct these electrolytes. Remarkably, the electrocardiogram showed no abnormalities, corresponding to hypokalemia and hypocalcemia. This observation, in combination with an overall increase in laboratory parameters with the exception of sodium and chloride, 
led to the suspicion of a pre-analytical error. Retrospectively, an intravenous catheter was inserted in close proximity of the puncture place but no continuous infusion was started prior to phlebotomy. However, the intravenous catheter was flushed with sodium chloride. Since potential other causes were excluded, the flushing of the intravenous catheter with sodium chloride prior to phlebotomy was the most probable cause for the deviating laboratory results and subsequently for the unnecessary potassium and calcium suppletion.

CONCLUSIONS: This case underlines the importance of caution in the interpretation of laboratory results obtained from specimens that are collected in the proximity of an intravenous catheter, even in the absence of continuous infusion.

Key Words: pre-analytical phase, phlebotomy, intravenous catheter, potassium, calcium

\title{
SECONDARY MGUS OR INTERFERENCE?
}

$\underline{\text { H. Bock }}^{2}$, F. Bühling ${ }^{1}$

${ }^{1}$ Zentrum für Laboratoriumsmedizin, Mikrobiologie und Krankenhaushygiene, Carl-Thiem-Klinikum Cottbus, Cottbus, Germany

${ }^{2}$ Zentrum für Laboratoriumsmedizin, Mikrobiologie und Krankenhaushygiene, Carl-Thiem-Klinikum Cottbus; School of Engineering and Physical Sciences, Heriot Watt University, Edinburgh, UK, Cottbus, Germany

BACKGROUND-AIM: As immunotherapies with monoclonal antibodies continue their success story, a fast growing number of therapeutic antibodies reach approval and advance to ever earlier stages of therapy. A good example is Daratumumab, which earlier this year gained approval by the FDA and the European Commission as first-line treatment for multiple myeloma patients. Naturally, the binding specificity that gives monoclonal antibodies their superior therapeutic value, makes them ideal for use in the medical laboratory.

METHODS: Blood samples tested via the indirect antiglobulin test, electrophoresis, immunofixation and flow cytometry.

RESULTS: Here we use the case of a 62-year-old patient with known multiple myeloma of class IgA and light chain lambda to discuss the diverse interferences of Daratumumab (IgG kappa) with various laboratory tests ranging from blood typing to flow cytometry. While workarounds have been developed for some tests, the key challenge for the laboratory is to know when to expect an interference. As antibody therapies are used far beyond the field of oncology and the realm of the hospital, therapeutic antibodies may be expected in any sample.

CONCLUSIONS: In our view effective communication between the physician and the laboratory, that should be simple but is so often challenging, is the key prerequisite to minimize the impact of antibody interferences.

Key Words. immunotherapies, antibody interferences

\section{SAMPLE PRE-WARMING BEFORE ANALYSIS REDUCES SPURIOUS IGM AND MONOCLONAL PROTEIN (MC) RESULTS}

\author{
P.C. Chan ${ }^{2}$, I.M. Blasutig ${ }^{1}$ \\ ${ }^{1}$ Children Hospital of Eastern Ontario \& University of Ottawa, Canada \\ ${ }^{2}$ Sunnybrook Health Sciences Centre \& University of Toronto, Canada
}

BACKGROUND-AIM: High immunoglobulin, particularly IgM, concentrations may increase sample viscosity and cause erroneous laboratory results through various mechanisms. We describe a case of Waldenstrom macroglobulinemia presented with spurious IgM and monoclonal protein (MC) results that could largely be averted by simple sample pre-warming at $37^{\circ} \mathrm{C}$ for $20 \mathrm{~min}$.

METHODS: A 58-year old Waldenstrom macroglobulinemic patient with no known cryoglobulin presented with spurious and conflicting IgM and M-protein results. Total protein and albumin were analyzed on the Abbott Architect while the IgM and MC were analyzed on the Siemens BNII and Sebia Hydrasys Gel Electrophoretic system respectively. Similar analyses were also performed on the Roche Modular and the Sebia Capillarys Electrophoretic systems in a separate facility.

RESULTS: Initial complaints from physician included jumpy IgM and MC concentrations that did not follow any pattern (varied from 10-32 g/L) and an estimated IgM MC concentration greater than total IgM e.g. 19 Vs $10 \mathrm{~g} / \mathrm{L}$ ). Sample aliquots sent to a referral lab (Lab1) using similar technology returned non-concordant results with the home lab e.g. IgM: 10 Vs 40 g/L and MC: 24 Vs 10. Subsequent repeat analyses of IgM and MC after sample pre-warming gave more consistent results (changed from IgM 7 to 19 and MC 4 to 25) which were in keeping with results (IgM 37 MC $22 \mathrm{~g} / \mathrm{L}$ ) obtained from a second referral lab (Lab2) that deployed different instrumentation but pre-warmed samples routinely.

CONCLUSIONS: Sample pre-warming at $37^{\circ} \mathrm{C}$ for $20 \mathrm{~min}$ appeared to be an effective means of avoiding spurious IgM and electrophoresis results, particularly from previously unknown and refrigerated samples.

Key Words: sample pre-treatment, fictitious results, immunoglobulin quantification, monoclonal gammopathy 


\title{
A SUDDEN RISE IN CLOT ERRORS FOR ROUTINE COAGULATION TESTING ON A SATURDAY MORNING
}

B. Calcoen $^{1}$, K. Desmet ${ }^{1}$, P. Vermeersch ${ }^{1}$

${ }^{1}$ Department of Laboratory Medicine, UZ Leuven, Leuven, Belgium

BACKGROUND-AIM: Several preanalytical problems can result in partially and/or fully clotted blood in citrate tubes including improper mixing of the tube, under- or overfilling or combining blood samples from different tubes into one citrate tube.

METHODS: A laboratory technician noted a sudden rise in the number of samples with clot errors on our track-coupled automated ACL TOP 700 analyzers (Instrumentation Laboratory Company, Bedford, MA). We describe how this preanalytical mystery was solved.

RESULTS: The sudden rise in clot errors prompted the technician to visually inspect the affected sample tubes. Several samples were completely solidified. In the tubes without visible clotting, several small clots were detected by inserting 2 wooden applicator sticks into the sample. Inspection also revealed that most of these citrate tubes originated from a few clinical departments. Moreover, after removing the barcode labels, it became clear that all affected tubes shared the same lot number. Further investigation revealed that, although 7 different lots of citrate tubes were in use in our hospital, 44\% of prolonged PT and aPTT results (52 of 118 results) were from tubes of the affected lot (including 19 results from tubes with a clot error). Instant action was taken by sending a warning message to the whole hospital to not use this lot of citrate tubes any longer. We controlled seven unopened boxes, each containing 100 citrate tubes of the affected lot. One box contained nine completely empty and two partially filled tubes and another box contained two partially filled tubes. No underfilled tubes were found in the other 5 boxes. The manufacturer undertook immediate action to recall the affected batch. The problem was most likely due to a problem at the start of the production of the batch. CONCLUSIONS: A sudden rise in clot errors should trigger a thorough investigation to identify the cause. A problem of additive volume in citrate tubes should be considered.

Key Words: preanalytical phase, blood coagulation test, diagnostic error, blood specimen collection

\section{WHEN CLOT DETECTION GOES WRONG: CONSEQUENCES AND COUNTERMEASURES FOR COMPLETE BLOOD COUNTS}

$\underline{\text { G. Frans }}^{2}$, P. Vermeersch ${ }^{2}$, I. Vandenbossche ${ }^{1}$

${ }^{1}$ Department of Laboratory Medicine, AZ Diest, Diest, Belgium

${ }^{2}$ Department of Laboratory Medicine, UZ Leuven, Leuven, Belgium

BACKGROUND-AIM: Clotted samples lead to spurious complete blood counts and should be rejected.

METHODS: We describe a patient in whom the automatic cell counter (Sysmex XE-5000) and laboratory algorithm failed to detect a large clot in a complete blood count sample.

RESULTS: A 75-year old women consulted the gastroenterologist for yearly follow-up of alcoholic liver cirrhosis and DM II. A routine blood investigation revealed anemia and thrombocytopenia (6,3 g/dL hemoglobin and 34x10 $/ \mathrm{L}$ PLT). No technical abnormalities or flags were noted on Sysmex XE-5000. Gastroscopy and CT thorax/abdomen revealed no underlying etiology. Iron substitution was initiated and 2 units of red blood cells were requested. The following day, before transfusion, a control sample revealed 9,1 g/dL hemoglobin and 72x10 $/ \mathrm{L}$ PLT. The transfusion was cancelled and an investigation of the discrepancy was initiated. Re-analysis of both samples gave similar results without flags. Visual inspection of the tubes revealed a large clot in the original sample, indicating falsely decreased values. The most likely cause of the failed clot detection on the automatic cell counter was inhomogeneous sample distribution (e.g. clot attached to top of tube). At the time, only PLT-morphology flagged samples (e.g. SIS rule 'PLT Clumps') triggered a clot detection algorithm. As a countermeasure, the post-analytical platelet SIS rules were reviewed and fine-tuned. All non-flagged thrombocytopenic samples (PLT $<140 \times 10^{9} / \mathrm{L}$ ) in patients with no prior history of thrombocytopenia or, in patients with known thrombocytopenia, with a positive PLT delta check (-34\% within 7 days) will now trigger a clot detection flag via the laboratory information system for visual inspection of the tube and a blood smear for aggregate detection.

CONCLUSIONS: Our case demonstrates the importance of detecting clots in complete blood count samples. Appropriate, laboratory-adapted rules should be able to counter this pre-analytical pitfall.

Key Words: clotted sample, automatic cell counter, complete blood count, laboratory algorithm

\section{HORMONES AFFECTED BY JET-LAG: A CASE REPORT}

\author{
M.S. Gungoren ${ }^{2}$, D.I. Topcu ${ }^{1}$, C. Zungun ${ }^{2}$ \\ ${ }^{1}$ Baskent University Ankara Hospital, Ankara, Turkey \\ ${ }^{2}$ Duzen Laboratories Group, Ankara, Turkey
}

BACKGROUND-AIM: As known today, all preanalytical conditions related with patient should be thoroughly interrogated. Sleeping status is one of the factors that can affect laboratory results. Intercontinental flights may lead to sleep disturbances (jet-lag) which can alter CNS 
hormones released from hypothalamus, pituitary and pineal gland. This case report is about the importance of timing and patient's metabolic status for hormone testing which arised from discordant results of a 34-year old male who was referred to sampling for serum TSH and PRL testing within 12-hour period after an intercontinental flight.

METHODS: A 34-year old male was admitted to our laboratory for serum TSH and PRL tests on a Monday morning.

RESULTS: His serum TSH level was $9.09 \mu \mathrm{IU} / \mathrm{mL}$ and his serum PRL level was $16.3 \mathrm{ng} / \mathrm{mL}$. On Saturday, he came back with a complaint on his previous results. He stated that he went to another hospital on Thursday and have discrepant results (TSH:3 $\mu$ IU/mL and PRL:9 ng/mL). Moreover, previous results were not compliant with his clinical condition. We explained that the samples were not the same and asked for any difference in his conditions. He stated no remarkable change in his lifestyle, etc. We offered him another analysis. His serum TSH was found as $2.8 \mu \mathrm{IU} / \mathrm{mL}$ and his serum PRL was found as $8.7 \mathrm{ng} / \mathrm{mL}$. We persistently tried to interrogate him about the differences between conditions prior to sampling. We asked about exercise, resting, any remarkable activity before sampling such as travelling. He confessed that the night before first sampling, he travelled back to Turkey from USA and came to testing within 12 hours after arrival. We explained him that intercontinental flight may have significant impact on CNS hormones.

CONCLUSIONS: Travel history and sleeping status are important parameters to be evaluated prior sampling for hormone analysis. Patients must be informed about importance of sampling timing.

Key Words: Preanalytical phase, case report, jet-lag, thyroid stimulating hormone, prolactin

\title{
HOW DOES THE IDENTIFICATION ERROR OCCUR DURING THE REQUESTING OF THE LABORATORY TESTS? A CASE REPORT FROM PEDIATRY CLINIC.
}

\author{
P. Eker ${ }^{1}$ \\ ${ }^{1}$ Istanbul Provincial Health Directorate, Chairman Of Public Hospital Services-2; Central Lab -2, Istanbul, Turkey
}

BACKGROUND-AIM: The preanalytical period is the most frequent error occurring part of all laboratory procedures. In various studies, the rate was reported as 56\%, 68.2 \%, and $61.9 \%$. The most critical sub-step in the preanalytical phase is the phlebotomy process and the riskiest part in phlebotomy is the identification of the patient. The aim of this case report is to understand the nature of an identification error and by this data to prevent the next ones.

METHODS: During our laboratory 'Key Performance Indicators' analysis, we noticed an identification error and then for making root cause analysis, we made a telephone call with the physician whose name was on the report and learned the details. First, he did not want to share the details and then we explained our aim was only preventing next ones. Then, he explained each step. We used the reports from LIS for the chronology of events and for obtaining the test results for both patients.

RESULTS: Case history: Baby A is a female and 3 months old and is being monitored for pancreatitis seconder to newborn sepsis in the newborn intensive care unit. Patient B is another 4 years old boy with a prediagnosis of lower respiratory tract infection and is being monitored in pediatry inpatient clinic. Identification error is made by the physician during requesting tests. Another clinician which wants to make his test requests for his patient noticed the error and then the clinician corrects the requesting error and informs the laboratory. The laboratory canceled all results and made a report.

CONCLUSIONS: In order to maintain patient safety, laboratory safety plays an important role and identification errors have the highest risk score in terms of patient safety. Patient and laboratory safety are both the basic objectives of quality management in healthcare. The most important outcome of every reported misidentification error case is each of them has a strong power to prevent next ones. The main responsibility of each healthcare facility is to manage identification errors.

Key Words: identification errors, patient safety, test requesting

\section{A CASE REPORT OF IDENTIFICATION ERRORS FOR TWO PATIENTS WITH SAME NAMES AND SAME AGES}

\author{
$\underline{\text { P. Eker }}^{1}$ \\ ${ }^{1}$ Istanbul Provincial Health Directorate, Chairman Of Public Hospital Services-2 ; Central Lab -2, Istanbul, Turkey
}

BACKGROUND-AIM: The preanalytical period is the most frequent error occurring part of all laboratory procedures. In various studies, the rate was reported as 56\%, 68.2 \%, and $61.9 \%$. The most critical sub-step in the preanalytical phase is the phlebotomy process and the riskiest part in phlebotomy is the identification of the patient. The aim of this case report is to understand the nature of an identification error and by this data to prevent the next ones.

METHODS: During our central laboratory 'Key Performance Indicators' analysis, we noticed an identification error which was made by the clinical nurse of the cardiology hospital which was sending samples to our central laboratory. We used the reports from LIS for details.

RESULTS: Case history: Patient A was a male, 58 years old and was being monitored with prediagnosis of peripheral vascular disease in heart and vascular surgery inpatient department of the hospital. Patient B was another male and 58 years old with a symptom of chest pain and was 
being monitored in the same clinic. Both of the patients were on the same ages and the first names of both were same. Identification errors were made by the nurse during phlebotomy. She had noticed the error and then called the laboratory. Laboratory canceled all the results and made a report.

CONCLUSIONS: In order to maintain patient safety, laboratory patient safety plays an important role. Patient identification errors have the highest risk score in terms of patient safety. Although at list two identifiers are suggested for confirming the procedure of phlebotomy some special situations two might be insufficient also.

Key Words: identification errors, patient safety, same names, same ages

\title{
PREANALYTICAL ERRORS IN CLINICAL LABORATORY: 3 CASE REPORTS
}

\author{
M. Pereira ${ }^{1}$, I. Cachapuz ${ }^{1}$ \\ ${ }^{1}$ Unidade Local de Saúde de Matosinhos, Matosinhos, Portugal
}

BACKGROUND-AIM: Preanalytical errors account for up to $70 \%$ of all mistakes made in laboratory diagnostics, most of which arise from problems in patient preparation, sample collection, transportation, and preparation for analysis and storage.

METHODS: We illustrate 3 cases studies to reinforce the importance of vigilance in preanalytical phase of testing.

RESULTS: Case report 1: A 47-year-old man, was hospitalized with a diagnosis of multiple myeloma. After the admission serum potassium was found to be $4.7 \mathrm{mmol} / \mathrm{L}$. The next day serum potassium measured from the sample was $6.6 \mathrm{mmol} / \mathrm{L}$. It was found that while blood collection in ward by nursing staff there was prolonged application of tourniquet. A tourniquet applied for prolonged periods of more than 1 minute causes hemoconcentration, altered water balance and hemolysis. Case report 2: A 72-year-old female patient showed an unexplained diurnal variation in hemoglobin value from $10.5 \mathrm{~g} / \mathrm{dl}$ to $13.1 \mathrm{~g} / \mathrm{dl}$. There was no clinical history of blood transfusion or hemoconcentration. A repeat sample was ordered and hemoglobin on repeat sample came out to be $10.2 \mathrm{~g} / \mathrm{dl}$. To find the cause of such variation we found that there was the difference in hemoglobin between two samples suggesting that the error was due to inappropriate or mislabeled vacutainer. Case report 3: A 8 months old male patient with fever was admitted with to the hospital, on day 1 there was an unexplained deviation in potassium $10 \mathrm{mmol} / \mathrm{L}$. The results were markedly deviated from previous day results with potassium $4.1 \mathrm{mmol} / \mathrm{L}$. On investigation, it was found that the plasma was obtained from blood collected in tripotassium EDTA tube.

CONCLUSIONS: Immediate, timely intervention and vigilance always prevent disastrous false results. As the errors in preanalytical phase are not inevitable and should be avoided with diligent application of quality control, education dedicated to patient care.

Key Words: laboratory errors, preanalytical phase, case reports

\section{TESTOSTERONE ABUSE AS A SOURCE OF PREANALYTICAL VARIATION OF PARAMETERS IN 24-HOUR URINE - A CASE REPORT}

\author{
$\underline{\text { D. Vuljanić }}^{1}$, V. Radišić Biljak ${ }^{1}$, E. Galić ${ }^{2,3}$, AM. Šimundić ${ }^{1}$ \\ ${ }^{1}$ Department of Medical Laboratory Diagnostics, University Hospital “Sveti Duh”, Zagreb, Croatia \\ ${ }^{2}$ Department of Internal Medicine, University Hospital “Sveti Duh”, Zagreb, Croatia \\ ${ }^{3}$ University of Zagreb, School of Medicine, Zagreb, Croatia
}

BACKGROUND-AIM: Various drugs and dietary supplements may temporarily affect nephrons and change urine constitution. We present a case of an outpatient with pathological $24 \mathrm{~h}$-urine findings incompatible with his clinical condition. Subsequent investigation revealed the reversible effect of testosterone abuse on parameters measured in $24 \mathrm{~h}$-urine sample, in this patient.

METHODS: A 41-year-old recreational male athlete was admitted to outpatient cardiology department due to hypertension and dizziness. Patient reported that he gained $30 \mathrm{~kg}$ in the past 5 months, regularly exercises and is on high-protein, low-carbohydrate and low-fat diet. Intake of other supplements was unknown at the time of the first $24 \mathrm{~h}$-urine collection. Routine biochemistry and quantitative urinalysis were performed on AU680 chemistry instrument (Beckman Coulter, USA).

RESULTS: Laboratory results indicated severe proteinuria (0,5 g), albuminuria (166 mg), highly elevated urea (2093 mmol), uric acid (20415 $\mu \mathrm{mol}$ ), creatinine $(71,6 \mathrm{mmol}$ ) and electrolytes (sodium $409 \mathrm{mmol}$, potassium $254 \mathrm{mmol}$, chloride $479 \mathrm{mmol}$, calcium $13 \mathrm{mmol}$, phosphorus $139,5 \mathrm{mmol}$ and magnesium $23,4 \mathrm{mmol}$ ) in $24 \mathrm{~h}$-urine sample. Patient also had extensive polyuria (5L/24h) and extremely high creatinine clearance $\left(412 \mathrm{ml} / \mathrm{min} / 1,73 \mathrm{~m}^{2}\right)$. Serum creatinine, urea, uric acid and electrolytes were within reference ranges. Findings in a repeated $24 \mathrm{~h}$-urine, 10 days after, were identical. Since $24 \mathrm{~h}$-urine findings were incompatible with patient clinical condition, possible sources of preanalytical errors were investigated. During thorough examination of personal patient history, patient has admitted the illegal use of testosterone, which explained his urine findings.

CONCLUSIONS: This case illustrates secondary changes in kidney function as a consequence of testosterone abuse. Understanding the effects of supplement intake is important for proper interpretation of laboratory results. 


\title{
IDENTIFICATION ERROR DETECTED BY THE SOFTWARE OF A FULLY-AUTOMATED HEMATOLOGIC ANALYZER
}

G.L. Salvagno ${ }^{1}$, F. Dima ${ }^{1}$, M. Montagnana ${ }^{1}$, G. Lippi ${ }^{1}$

${ }^{1}$ Section of Clinical Biochemistry, University Hospital of Verona, Verona, Italy

BACKGROUND-AIM: Identification errors may generate serious harms to the patients, even when involving laboratory testing. We describe here the case of an identification error detected by the software a fully-automated hematologic analyzer.

METHODS: During routine workout in the hematologic section of the local laboratory, a whole blood sample was flagged by the analyzer (Sysmex XN; Sysmex Co, Kobe, Japan) due to thrombocytopenia (i.e., 36x10\%/L).

RESULTS: According to internal rules set in the software of the analyzer, an automatic rerun was activated for confirming platelet data. The results of this second test were completely different from the former, i.e., platelet count $297 \mathrm{vs} 36 \times 10^{9} / \mathrm{L}$, leukocytes $13.37 \mathrm{vs} 4.28 \times 10^{9} / \mathrm{L}$, erythrocytes 3.51 vs 3.00×10 $12 / \mathrm{L}$ and hemoglobin $109 \mathrm{vs} 95 \mathrm{~g} / \mathrm{L}$. Upon second rerun, data confirmed those of the first rerun. Due to these major discrepancies, the laboratory staff visualized number and position of the sample on the middleware, realizing that initial testing was performed on a sample positioned in rack 35 position 4, whilst the two ensuing reruns were performed on a sample positioned in rack 23 position 9. Further inspection of samples revealed that two separate specimens, with identical labels, have been loaded into the analyzer. The results of the second sample were initially disregarded by the analyzer due to presence of first sample data, but rerun was automatically performed by the analyzer on the second sample. The second sample was finally attributed to another patient, whose blood was collected into a blood tube labeled with the ID of the first patient. A new rule was hence set in the software of the analyzer, according to which duplicate samples will now be flagged before being processed.

CONCLUSIONS: Identification errors continue to occur in healthcare and frequently involve laboratory diagnostics. Availability of efficient and foolproof IT tools appears crucial for their timely identification.

Key Words: identification errors, hematologic analyzer, preanalytical phase

\section{DEALING WITH INTERFERENCES CAUSED BY M-PROTEIN}

\author{
$\underline{\text { A. Dojder }}^{2}$, D. Vuljanić ${ }^{2}$, I. Kuktić ${ }^{2}$, D. Šegulja ${ }^{1}$, N. Nikolac Gabaj ${ }^{3}$, V. Radišić Biljak ${ }^{2}$, AM. Šimundić ${ }^{2}$ \\ ${ }^{1}$ Department of Laboratory Diagnostics, University Hospital Centre Zagreb, Zagreb, Croatia \\ ${ }^{2}$ Department of Medical Laboratory Diagnostics, University Hospital “Sveti Duh", Zagreb, Croatia \\ ${ }^{3}$ University Department of Chemistry, Medical School, University Hospital Sestre milosrdnice, Zagreb, Croatia
}

BACKGROUND-AIM: M-proteins are endogenous interferents. Although M-protein interference has long been recognized, detection and management of samples affected by M-protein interference remains a challenge for laboratories worldwide. Here we present a case of M-protein interference on direct bilirubin measurement and how to deal with it in order to report accurate and reliable results to the requesting physician.

METHODS: A 76 year old female patient with a previously known diagnosis of multiple myeloma has had her regular check-up. Her serum proteins were $95 \mathrm{~g} / \mathrm{L}$ at the time. Concentrations of direct and total bilirubin analyzed by AU860 (Beckman Coulter, USA) analyzer were -12.3 and $8.5 \mu \mathrm{mol} / \mathrm{L}$, respectively. Since M-protein interference was suspected, sample was diluted with distilled water (1:10 ratio) to gain the lower concentration of M-proteins, treated with PEG to precipitate them, and treated with HBT to exclude the heterophilic interference. Once interference was confirmed, sample was sent to two other laboratories and analyzed on two different analyzers: Cobas 6000 (Roche, Switzerland) and Architect c8000 (Abbott, USA).

RESULTS: The direct bilirubin concentration in diluted sample was $3.0 \mu \mathrm{mol} / \mathrm{L}$. It was $4.0 \mu \mathrm{mol} / \mathrm{L}$ after PEG precipitation and $-2.0 \mu \mathrm{mol} / \mathrm{L}$ after HBT treatment. Direct bilirubin concentration on different platforms were as follows: $1.6 \mu \mathrm{mol} / \mathrm{L}$ on Cobas 6000 and $2.8 \mu \mathrm{mol} / \mathrm{L}$ on Architect, revealing no interference.

CONCLUSIONS: In case when the interference of M-proteins is suspected, PEG precipitation and sample dilution with distilled water is sufficient to confirm interference. If accurate result is necessary, sample should be analyzed on a different instrument where interference is not present. HBT treatment is not effective in eliminating M-protein interference. Communication with a patient and a requesting physician is vital for proper management of this type of interference.

Key Words: preanalytical phase, bilirubin, interference, multiple myeloma, M-protein

\section{PATIENT IDENTIFICATION ERROR. A PARTICULAR CASE}

$\underline{\text { S. Pico }}{ }^{1}$, M. Duque ${ }^{1}$, M. Ibarz ${ }^{1}$, E.E. Moreno ${ }^{1}$, M. Caballero ${ }^{1}$, M. Llovet $^{1}$, J. Prieto ${ }^{1}$, M. Dolade ${ }^{1}$, E. Marquez ${ }^{1}$, L.M. Cruz ${ }^{1}$, N. Del Amo ${ }^{1}$, F.J. Merida ${ }^{1}$

${ }^{1}$ Committe for the Patient Safety of the Spanish Society of Laboratory Medicine (SEQC-ML), Spain 
BACKGROUND-AIM: Patient identification error is one of the biggest challenges in clinical laboratory linked to patient safety. The consequences of this error include potential misdiagnosis, inappropriate treatments, and could be associated with adverse events. We present a case of identification error involving two patients.

METHODS: A 78-year old male with dilated cardiomyopathy, auricular fibrillation, chronic kidney failure, type II diabetes and arterial hypertension went to his phlebotomy center with three different analytical orders. The laboratory received two samples from this patient (P1) with two different laboratory numbers (L1, L2). Results were reviewed using delta check tool. Analytical results showed important discrepancies. L1: Normal protein serum electrophoresis, NT- ProBNP 8,856 pg/mL and altered kidney function. L2: presence of serum monoclonal protein, NT-ProBNP $27.4 \mathrm{pg} / \mathrm{mL}$ and normal kidney function. Laboratory specialist thought in two different patients and a probably identification error. Analysis of Causes, Weaknesses, and Barriers was carried out.

RESULTS: Causes: only two of the three P1 orders were identified, the third one was missing. This order was identified as Patient 2 (P2). Weaknesses: The information system does not have an automatic mechanism for merging requests. Barriers: Error in the identification barrier, P2 was not properly identified. Delta check barrier allowed detecting the error. Clinical laboratory specialist cancelled both orders, so the two patients had to repeat the blood withdrawal.

CONCLUSIONS: The reactive analysis allows us to identify which is the error to establish different barriers in order to prevent future adverse events.

One of the most important clinical laboratory challenges should be create an information and training loop between the laboratory and the blood collection centers in order to stop or minimize this type of errors.

Key Words: identification error, pre-analytical phase, patient safety

\title{
SPURIOUSLY LOW SODIUM AND CALCIUM IN A 36 YEAR OLD MALE IN PRIMARY CARE
}

\author{
$\underline{\text { S. Costelloe }}{ }^{1}$, K. Mccarthy ${ }^{1}$, M. O'connell ${ }^{1}$, M. Butler ${ }^{1}$ \\ ${ }^{1}$ Department of Clinical Biochemistry, Cork University Hospital (CUH), Wilton, Cork, Republic of Ireland
}

BACKGROUND-AIM: Identifying serum specimens with delayed separation requires information on collection time. When these data are unavailable or incorrect, artefactual or misleading test results may be reported.

METHODS: An unseparated gel serum tube for a 36 year old male with unknown medical history was received at CUH from primary care. The request card indicated phlebotomy was the previous day. The sample was centrifuged and run on a Beckman Coulter AU5800 for tests including serum indices, sodium (Na), potassium (K), aspartate transaminase (AST), total bilirubin (TBIL), and total calcium (tCa).

RESULTS: A haemolysis index of 2 blocked results for K, AST, and TBIL. Na was $119 \mathrm{mmol} / \mathrm{L}$ and tCa was $1.15 \mathrm{mmol} / \mathrm{L}$ and both breached departmental critical phoning limits. Senior staff noted that results might have been consistent with delayed separation. The General Practitioner (GP) confirmed the request card had been completed incorrectly and that phlebotomy was actually 7 days prior to specimen receipt in the laboratory. CONCLUSIONS: Preanalytical errors can converge and lead to confusion in the generation and reporting of laboratory results. The authors show how inaccurate date and time of collection led to a confusing picture where it was not immediately apparent that centrifugation of a specimen had been significantly delayed. Elevations of K are often an indicator of potential delayed separation in serum specimens. However, in this case, the $\mathrm{K}$ was not reported due to the degree of haemolysis. Spuriously low tCa and Na can results where separation is delayed by $>2$ days, or $>4$ days, respectively. In this case, laboratory vigilance and discussion with the GP prevented inappropriate patient management. Had results been generated out-of-hours, they may have been phoned to the on-call clinical service provider in the region, rather than the requesting GP. In such as scenario, there is a risk that the patient would have been inappropriately referred to the Emergency Department.

Key Words: hyponatraemia, hypocalcaemia, delayed separation

\section{CASE REPORT OF A PHANTOM PHAEOCHROMOCYTOMA}

\author{
$\underline{\text { C. Joyce }}^{1}$, A. Melvin ${ }^{2}$, S. Costelloe ${ }^{1}$, D. O'halloran ${ }^{2}$ \\ ${ }^{1}$ Clinical Biochemistry Department, Cork University Hospital \\ ${ }^{2}$ Endocrinology Department, Cork University Hospital
}

BACKGROUND-AIM: Phaeochromocytoma is a rare neuroendocrine disorder associated with hypertension and hypersecretion of catecholamines. We present the case of a 53 year old lady with a history of hypertension and left adrenalectomy. Medical records were unavailable to confirm phaeochromocytoma but annual biochemical screening was commenced to check for recurrence.

METHODS: Biochemical tests included renin, aldosterone and chromogranin A levels. Three consecutive 24hour urine collections were analysed for fractionated metanephrines by HPLC/ECD and LC-MS/MS. Fasting plasma free metanephrines were also analysed by LC-MS/MS.

RESULTS: Biochemical results for renin, aldosterone and chromogranin A were all normal. Normetanephrine was grossly elevated in all three urine collections analysed by HPLC/ECD, at 35237, 19440 and $5813 \mathrm{nmol} / 24 \mathrm{hr}$ respectively (RR <2800). Plasma normetanephrine (1156 pmol/L) was within normal limits (RR 120-1180). Phaeochromocytoma was strongly suspected based on past history and elevated normetanephrines. CT imaging with contrast was performed on the right adrenal, thorax, abdomen and pelvis. A whole body octreotide scan was also performed 
but no abnormality was detected. Repeat normetanephrine results by LC-MS/MS were normal at 2924, 3978 and $3864 \mathrm{nmol} / 24 \mathrm{hr}$ respectively $(\mathrm{RR}<4900)$.

CONCLUSIONS: Concerns were raised about the initial normetanephrine results which were not confirmed by LC-MS/MS. Analytical drug interference was suspected and patient medications were reviewed. Sulfasalazine was identified as the most likely culprit as it is known to cause falsely elevated normetanephrine and interfere in electrochemical detection (ECD) (Lenders, 2014). An erroneous normetanephrine result led to numerous unnecessary expensive imaging procedures. This case highlights the importance of taking a good drug history and critically reviewing all test results before diagnosing phaeochromocytoma.

Key Words: phaeochromocytoma, catecholamines, metanephrines, sulfasalazine

\title{
THREE UNCOMMON CASES OF PREANALYTICAL PROBLEMS IN THE CLINICAL LABORATORY OF THE FACULTY HOSPITAL
}

\author{
M. Bunesova $^{2}$, B. Friedecky ${ }^{1}$ \\ ${ }^{1}$ Faculty Hospital,Hradec Králové, Czech Republic \\ ${ }^{2}$ Faculty Hospital Prague-Motol, Czech Republic
}

BACKGROUND-AIM: Case studies of wrongly identified samples, bad timing of sampling and wrongly performed sampling METHODS: description of cases, communication, precautions

RESULTS: Case 1: Wrong identification of samples-The analysis of a serum sample of a patient treated with lamotrignin gave unexpected results above the upper reference interval of urea and creatinine. A swap of barcodes on samples from two patients was found. The problem was solved by an identification correction and later issuance of the correct results. The mistake was caused by a member of the staff of the laboratory. There is a need to automate the preanalytical phase further to eliminate human error as much as possible. Case 2: Bad timing of sampling and lack of communication. A patient who had undergone a bone marrow transplant had in his first sampling an extremely high concentration of ciclosporin A that did not correspond with a steady state.When a second sampling was made, a decrease by 8-9 times was discovered. No significant analytical mistake was found. We believe the mistake was caused by wrong timing of the first sampling that is the sampling was not made in a steady state. The reaction of the ward was inadequate and needed solving by the heads of laboratories and wards. Case 3: Risk sampling for compensation check of diabetic patients HbA1c. A greater amount of capillary sampling when checking diabetic patients lead to a discussion with the ward. It was found, that the capillary sampling was done in a way that endangered the health of the staff. The ward wanted to solve the situation by the use of POCT even though the need is fully fulfilled by a well working and certified laboratory method. This problem is, due to its significance, being solved by the hospital top management.

CONCLUSIONS: The stated cases show that identification mistakes can appear at anytime and anywhere, that the cooperation with wards is not always ideal and that there are cases were the health of the staff is put in risk.

Key Words: preanalytical phase

\section{Sample collection}

\section{COMPARISON OF CLINICAL BIOCHEMISTRY TESTS WITH LI-HEPARIN (BARRICOR TM) AND GEL TUBES}

\author{
S. Kader ${ }^{1}$, M. Ercan ${ }^{2}$ \\ ${ }^{1}$ Karapinar Public Hospital Biochemistry Laboratory Konya, Turkey \\ ${ }^{2}$ Harran University, Faculty of Medicine, Medical Biochemistry, Sanliurfa, Turkey
}

BACKGROUND-AIM: Reliable and rapid test results requested by clinic departments are important for the patient to receive early treatment, as well as to shorten staying hospital.Since use of plasma needs no clothing time and shortness the centrifugation, we compared BD Vacutainer® Barricor ${ }^{\mathrm{TM}}$ lithum heparin tubes with mechanical separator for the chemistry parameters with our current tubes; BD Vacutainer ${ }^{\circledR}$ serum gel tubes

METHODS: Blood samples taken from randomly selected 36 patients collected to lithium heparin (Barricor ${ }^{\mathrm{TM}}$ ) and serum gel tubes. Barricor тм tubes were centrifuged for 3 minutes at $2360 x g$, and serum gel tubes were centrifuged for 10 minutes at $1500 x g$. Twenty two chemistry parameters were analysed. Dependent $t$ test was used for normal distribution tests and Wilcoxon sign test was used for non-normal distribution. Mean, Bias and \% bias were applied for clinical significancy level.

RESULTS: No statistically significant difference found for the any parameters results and no clinically significant difference was detected for any parameters. Accordingly, for all analyte results that we have worked on, there is an agreement between SST ${ }^{\mathrm{TM}} \mathrm{II}$ and Barricor ${ }^{\mathrm{TM}}$ within the CAP and CLIA values. 
CONCLUSIONS: As a result, there were no statistically significant differences in any parameters between the BD Vacutainer® Barricor ${ }^{\mathrm{TM}}$ plasma tube with mechanical seperator and the serum gel tubes. The BD Vacutainer ${ }^{\circledR}$ Barricor ${ }^{\mathrm{TM}}$ plasma tube can be an alternative to serum gel tubes to shorten the processing time (TAT) and improve sample quality.

Key Words: Barricor, Li-heparin, serum gel tube, mechanical separator, plasma tube

\title{
ASSESSING ERYTHOCYTESEDIMENTATION RATE IN WHOLE BLOOD ANTICOAGULATED WITH SODIUM CITRATE OR K2EDTA
}

\author{
G.L. Salvagno ${ }^{1}$, F. Dima ${ }^{1}$, L. Salandini ${ }^{1}$, G. Lippi ${ }^{1}$ \\ ${ }^{1}$ Section of Clinical Biochemistry, University Hospital of Verona, Verona, Italy
}

BACKGROUND-AIM: According to the International Council for Standardization in Haematology (ICSH), Westergren method is the reference technique for measuring erythocytesedimentation rate (ESR). Although this assay has been originally validated on whole blood anticoagulated with sodium citrate, the use of whole blood anticoagulated with $\mathrm{K}_{2}$ EDTA is now commonplace for many practical and technical reasons, and is also acknowledged by the ICSH. This study was hence planned to define how ESR values would compare between these two sample matrices.

METHODS: A total number of 148 consecutive paired blood samples collected either in $3.5 \mathrm{~mL}, 3.2 \%$ trisodium citrate (Vacutest Kima, Padova, Italy) and $3.0 \mathrm{~mL}, 5.4 \mathrm{mg} \mathrm{K}$ EDTA (Vacutest Kima, Padova, Italy) were randomly selected from all routine samples arrived on a working day in the local laboratory for routine hematologic and hemostasis testing. The ESR was measured on all uncentrifuged samples, using the same fully automated analyzer TEST 1 (Alifax, Padova, Italy).

RESULTS: The median (and interquartile range; IQR) ESR value was more than double in K,EDTA ( $36 \mathrm{~mm} / \mathrm{h}$; IQR, $56 \mathrm{~mm} / \mathrm{h}$ ) than in citrate $(17 \mathrm{~mm} / \mathrm{h}$; IQR, $41 \mathrm{~mm} / \mathrm{h}$ ) tubes (Mann-Whitney paired test, $\mathrm{p}<0.001)$. The Spearman's correlation and mean difference of $\mathrm{K}_{2}$ EDTA vs sodium citrate, calculated with Bland \& Altman plots, were $\mathrm{r}=0.87$ (95\% CI, 0.83-0.91; p<0.001) and 61\% (95\% CI, 52-70\%). In 111/148 samples (i.e., $75 \%$ ) the difference was $>5 \mathrm{~mm} / \mathrm{h}$, thus not fulfilling the ICSH criterion that $95 \%$ of the differences observed between alternative testing conditions should be $\leq 5 \mathrm{~mm} / \mathrm{h}$. Finally, the percentage of samples with ESR values $>20 \mathrm{~mm} / \mathrm{h}$ was significantly higher in $\mathrm{K}_{2}$ EDTA than in sodium citrate samples ( $66 \%$ vs 47\%; Chi-squared test, $\mathrm{p}=0.001)$.

CONCLUSIONS: Albeit both $\mathrm{K}_{2}$ EDTA and citrate tubes can be currently used for measuring ESR, results are not interchangeable and specific reference ranges should be adopted for these two sample matrices.

Key Words: erythrocytesedimentation rate, sodium citrate, K_EDTA, preanalytical phase

\section{PLASMA SAMPLE QUALITY: COMPARISON BETWEEN BARRICOR® AND BD LITHIUM HEPARIN}

J.A. Delgado RodrÍguez ${ }^{1}$, M.A. Ballesteros Vizoso ${ }^{1}$, P. Argente Del Castillo ${ }^{1}$, M.M. Parera RossellÓ ${ }^{1}$, A.R. Pons Mas ${ }^{1}$, I. Llompart Alabern ${ }^{1}$, J.M. BauÇa ${ }^{1}$

${ }^{1}$ Department of Laboratory Medicine, Hospital Universitari Son Espases, Palma de Mallorca, Spain

BACKGROUND-AIM: Towards higher patient safety, the ISO 15189:2012 accreditation requires a continuous improvement in the quality management of the clinical laboratory. In this context, tubes for blood collection represent a key factor in the preanalytical phase, although sometimes underestimated.A new blood collection device has been recently developed for plasma samples: the Vacutainer Barricor ${ }^{\circledR}$ (Becton Dickinson), which could improve sample quality, magnitude stability and centrifugation time. In order to reduce the percentage of hemolyzed samples (defined as hemoglobin $\geq 50 \mathrm{mg} / \mathrm{dL}$ ) in our Stat Laboratory, the new BD Barricor ${ }^{\circledR}$ tubes was assessed and compared with the currently used lithium heparin (LH) under the usual working conditions.

METHODS: A total of 77 patients admitted in the ICU were recruited. Blood samples were obtained in duplicate from central lines: one in a Barricor® tube, and the other one in LH. Samples were brought to the laboratory and immediately centrifuged for $5 \mathrm{~min}$ at $1400 \mathrm{~g}$. Up to 20 ISO-accredited biochemical parameters were measured on the Architect c16200 platform (Abbott Diagnostics), as well as the hemolysis index (HI), which represents one of the most used preanalytical quality indicators in laboratory medicine. The Kolmogorov-Smirnov test was used to assess normality, and comparisons were performed using the Student's t-test or the Mann Whitney's U-test. Statistical significance was set at 0.05. The SPSS v.24 software was used for all statistical analyses.

RESULTS: Magnitudes with statistically significant differences between both tubes were HI $(\mathrm{p}<0.001)$ and Potassium ion $(<0.001)$.

CONCLUSIONS: Under the working conditions in our laboratory, the new BD Barricor ${ }^{\circledR}$ tube shows a higher red blood cell lysis than the current plasma lithium heparin tubes, thus releasing the intra-erythrocyte components and yielding abnormal results of hemolysis-related parameters. However, the clinical impact of such difference needs further study.

Key Words: blood collection tubes 


\title{
PREANALYTICAL CONSIDERATIONS OF URINE TROPONIN I ANALYSIS - A CASE REPORT
}

\author{
L. Culej ${ }^{1}$, M. Božović ${ }^{1}$, A. Vrtarić ${ }^{1}$, A. Topić ${ }^{1}$, L. Milevoj Kopčinović ${ }^{1}$, N. Nikolac Gabaj ${ }^{1}$ \\ ${ }^{1}$ Department of clinical chemistry, Sestre milosrdnice University Hospital Center, Zagreb, Croatia
}

\begin{abstract}
BACKGROUND-AIM: Serum high sensitivity troponin I is measured in serum. However, some studies have assessed its potential in first morning urine sample. Despite intriguing results, preanalytical factors should be considered before any clinical use. It was also noticed that troponin levels may be increased after high endurance exercise. The aim of our study was to investigate hsTnI level in urine sample during 7 day period which included wide range of physical activity.

METHODS: Urine hsTnI and creatinine levels have been monitored in 35 years old female recreational runner during 7 day period (Tuesday to Monday). hsTnI and creatinine levels were analyzed in first morning urine sample on Abbott i2000 analyzer and Abbott c8000 respectively. Tuesday (day 1): sample without prior physical activity. $5 \mathrm{~km}$ training in the evening. Wednesday (day 2): sample after activity. Thursday (day 3): sample without physical activity. 4,41 km in the evening. Friday (day 4): sample after physical activity. Saturday (day 5): $18 \mathrm{~km}$ trail race. Sample was taken 4 hours after finishing the race. Sunday (day 6): first morning sample stored at $4^{\circ} \mathrm{C}$, hsTnI was measured next day. Monday (day 7): first morning sample.

RESULTS: hsTnI (ng/L) and creatinine (mmol/L) levels on day 1 were $27 \mathrm{ng} / \mathrm{L}$ and 6.3mmol/L; day 2: 8ng/L and 10.4mmol/L; day 3: 9ng/L and 7.9mmol/L; day 4: 13ng/L and 9.5mmol/L ; day 5: 48ng/L and 10.6mmol/l; day 6: 0ng/L and 16.3mmol/L; day 7: 4ng/L and 5.6mmol/L. hsTnI/ creatinine ratio was: $4.29 ; 0.77 ; 1.14 ; 1.37 ; 4.53 ; 0 ; 0.71$ respectively. Urine sediment analysis revealed presence of bacteria in samples after race. CONCLUSIONS: The highest hsTnI level was measured in a sample after race. However, hsTnI/creatinine ratio was similar to the ratio on the first day without prior increase in physical activity, suggesting effect of hydration on troponin level in urine. hsTnI was measurable in every sample except for sample 6, analyzed the next day suggesting possible degradation of hsTnI in urine, possibly due to presence of bacteria.
\end{abstract}

Key Words: urine, troponin I, physical activity

\section{THE IMPLEMENTATION OF THE BD BARRICOR AND THE EFFECTS ON TAT AND LABORATORY RESULTS: THE EXPERIENCE OF AN ONCOLOGY WARD}

\author{
A. Padoan ${ }^{1}$, E. Piva ${ }^{1}$, M. Zaninotto ${ }^{1}$, L. Sciacovelli ${ }^{1}$, A. Tasinato ${ }^{1}$, M. Plebani ${ }^{1}$ \\ ${ }^{1}$ Department of Laboratory Medicine, University-Hospital of Padova, Italy
}

BACKGROUND-AIM: Clinical care pathways to manage oncologic patients therapies require blood sampling check to avoid adverse outcomes. The process requires timeliness and short turnaround times (TAT) for benefit patients with respect to therapeutic needs. The aim of this study was to assess the impact of reducing the preanalytical centrifugation time, by implementing the BD Vacutainer® Barricor ${ }^{\text {TM }}$ Plasma collection tube (Barricor) with respect to BD PSTII plasma tube.

METHODS: Centrifugation was made using 3000gx5 min with BD PSTII tubes and 4000gx3 min with Barricor. Creatinine TAT was evaluated. Paired patients' results for ALT, Calcium $\left(\mathrm{Ca}^{2+}\right)$, Potassium $\left(\mathrm{K}^{+}\right)$, Sodium $\left(\mathrm{Na}^{+}\right)$, Creatinine (Crea), total bilirubin (tBil) and Urea values were also evaluated seven months before (Jan to July 2017) and after (Jan to July 2018) the implementation of Barricor tubes (Sept 2017). Bland Altman and Log-rank tests were performed using MedCalc Software version 18.2.1.

RESULTS: A total of 4804 patients' results were evaluated. Median/90 th centile TAT values were reduced from $26 \mathrm{~min} / 45 \mathrm{~min}$ before, to $25 \mathrm{~min} / 38 \mathrm{~min}$ after Barricor tubes implementation (log-rank test $\mathrm{p}<0.001$ ). For each patient, a biochemical monitoring has been carried out, showing a mean of 6.4 determinations using PSTII and 6.1 using Barricor tubes and the median values of tests results has been calculated. After the implementation of BD Barricor, the mean bias $\%$ on obtaining results were: ALT: $-6.3(\mathrm{p}<0.01)$; $\mathrm{Ca}^{2+}:-0.22(\mathrm{p}=0.218), \mathrm{K}^{+}: 1.0$ $(\mathrm{p}<0.01), \mathrm{Na}^{+}:-0.10(\mathrm{p}<0.147)$, Crea: $1.22(\mathrm{p}<0.01)$, tBil: $8.7(\mathrm{p}<0.01)$ and Urea: $1.8(\mathrm{p}=0.114)$. The statistically significant biases were all clinically acceptable, being smaller than the desirable bias on the basis of biological variation (https://www.westgard.com/biodatabase1.htm for ALT, EuBIVAS study, EFLM for the others).

CONCLUSIONS: At oncology care setting, Barricor tubes positively impact laboratory TAT, improving the management of patients therapy.

Key Words: turnaround time, blood collection tubes, barricor plasma tube, oncology ward

\section{FREQUENCY OF LATENT FIBRIN IN CENTRIFUGED SERUM SPECIMENS.}

$\underline{\text { W. Sharif }}^{1}$, A. Stretton ${ }^{2}$, M. Cornes ${ }^{1}$, C. Shetty ${ }^{1}$

$\left.{ }^{1} 1\right)$ Biochemistry Department, Worcestershire Acute Hospitals NHS Trust, Worcester, UK

22) Becton, Dickinson and Company, Winnersh, UK

BACKGROUND-AIM: Fibrin clots are formed in serum specimens when not allowed to adequately clot prior to centrifugation resulting in latent fibrin formation in the serum. Fibrin clots may cause erroneous results via short samples, assay interference, and can block analyser probes, leading 
to subsequent mis-sampling or analyser downtime. Fibrin artefacts may occur as small fibrous structures, as well as larger visible clots. All erroneous fibrin structures have the potential to cause analytical issues, but the smaller fibrin structures are much harder to detect in routine diagnostic testing. Inadequate clotting pre-processing may be due to insufficient time to clot, anticoagulant interference or inadequate specimen mixing.

METHODS: We audited 57 blood collections from 4 different locations in the Worcestershire Royal Hospital for the frequency of latent fibrin. Phlebotomy observations were measured using a Preanalytical Quality Check (PAQC) software tool (Becton, Dickinson and Company) including time of sampling, transport method, time of receipt in the laboratory, time of centrifugation and visual inspection of the specimen for the presence of fibrin after centrifugation.

RESULTS: $2 \%$ of samples had no mixing of specimens. Specimens received via pneumatic tube were on average processed and reported 40 minutes faster than hand-delivered samples. $12 \%$ of serum specimens had clots or other signs of latent fibrin. All samples with latent fibrin were from the Intensive Care Unit (ICU) and the majority were analysed in the morning.

CONCLUSIONS: Samples centrifuged prior to completion of manufacturers' clotting time recommendations are more prone to form latent fibrin. Here we have shown that samples that are from a prioritised location, samples from patients more likely to be on anticoagulants and those analysed at a low throughput time of day are more likely to have latent fibrin. Latent fibrin has an impact on the quality and costefficiency of laboratory results.

Key Words: fibrin, pre-analytical, serum, centrifugation

\title{
COMPARISON OF HAEMOLYSIS RATES BETWEEN SAFETY BLOOD COLLECTION SETS AND SAFETY TUBE HOLDERS IN A BUSY HOSPITAL PHLEBOTOMY SERVICE
}

\author{
$\underline{\text { N. Andrade }}{ }^{2}$, N. Rico Rios ${ }^{1}$, K. Mccarthy ${ }^{1}$, R. Mulcahy ${ }^{2}$, D. Forde ${ }^{2}$, H. Morton ${ }^{2}$, J. Safaric ${ }^{2}$, S. Creagh ${ }^{1}$, L. Heeney ${ }^{2}$, S. Costelloe ${ }^{1}$ \\ ${ }^{1}$ Department of Clinical Biochemistry, Cork University Hospital, Wilton, Cork, Republic of Ireland \\ ${ }^{2}$ Phlebotomy Department, Cork University Hospital, Wilton, Cork, Republic of Ireland
}

BACKGROUND-AIM: Experience at the Phlebotomy Department of Cork University Hospital (CUH) suggested that use of Greiner Bio-One Safety Blood Collection Sets + Holder ((SBC), needle bore of 23G) during phlebotomy lead to a higher haemolysis rate (HR) than when the VACUETTE® QUICKSHIELD Safety Tube Holder ((STH), needle bore of 21G) was used. Literature is lacking on this comparison and data on the effects of needle bore on HR are conflicting. This study compares the HR observed when using SBC and STH.

METHODS: This consecutive observational study analysed data for specimens taken by fully trained phlebotomists on renal, surgery, and medical wards between 3rd and 28th September 2018. SBC was used when patients had difficult access or were dehydrated. For all other patients, STH was used. Specimens were transported by porter to the laboratory and analysed on a Beckman AU5800 analyser with Haemolysis indices ((HI), graded 0-6) assessed by spectrophotometry. HR data were described and Chi-square analysis $(\mathrm{P}<0.05)$ used to determine if HR between the devices were significantly different.

RESULTS: From 128 patients, 317 samples were considered, with 130 (41\%) collected using SBC and 187 (59\%) collected by STH. Haemolysis data were missing in 2 cases. Eleven specimens (3.5\%) had HI $>0$ ( 8 with HI1, 3 with HI2). The difference in HR between devices was not statistically significant $(\mathrm{SBC}=3.8 \%, \mathrm{STH}=3.2 \%$, Chi-square $=0.083, \mathrm{P}=0.77)$ and HI1 was more common than HI2 for both devices. For the 34 patients (27\%) who had more than 1 specimens taken by each device: 29 showed HIO regardless of device; 2 had HI >0 with STH only; 2 showed $\mathrm{HI}>0$ regardless of device; and 1 had had $\mathrm{HI}>0$ with $\mathrm{SBC}$ only.

CONCLUSIONS: There was no difference between HR, or degree of haemolysis, when using SBC or STH. Neither met the American Society for Clinical Pathology benchmark of $\mathrm{HR} \leq 2 \%$. A larger sample size is required to assess the effect of user, patients' clinical status, or demographics on HR.

Key Words: haemolysis, blood collection, needle bore

\section{COMPARISON OF TWO SERUM COLLECTION TUBES FOR THYROID AUTOANTIBODIES DETERMINATION}

\author{
$\underline{\text { D. Stošić Đurić }}^{1}$, S. Božičević ${ }^{1}$, M. Krhač ${ }^{1}$, M. Vučić Lovrenčić ${ }^{1}$ \\ ${ }^{1}$ Clinical Department of Medical Biochemistry and Laboratorv Medicine, Merkur University Hospital, Zagreb, Croatia
}

BACKGROUND-AIM: Selection and verification of blood collection tubes is an important preanalytical issue in clinical laboratories. Our aim was to compare thyroid antibodies concentration in sera collected in BD CAT and SST II Advanced tubes and evaluate the equivalence of results for the clinical use.

METHODS: Venous blood from 42 subjects who came for thyroid testing was collected using both BD CAT and SST tubes in sequence. Sera from both tubes, obtained after clotting and centrifugation (15 min, 1000xg) were assayed for anti-Tg and anti-TPO autoantibodies with an automated chemiluminescent immunoassay (Centaur XP, Siemens, USA). The results were analyzed using MedCalc statistical software.

RESULTS: Serum anti-Tg levels ranged from 15 to $500 \mathrm{kU} / \mathrm{L}$. Passing \& Bablok regression analysis revealed no statistically significant systematic or proportional differences between the two compared collection tubes (regression equation: $y=-0,993941+1,017722 x$; Intercept 
$A=-0,9939 ; 95 \% C I=-2,4801$ to 1,9735; Slope $B=1,0177 ; 95 \% C I=0,9961$ to 1,0722$)$. Cusum test for linearity showed no significant deviation from linearity $(\mathrm{P}=0,56)$. Weighted kappa coefficient was 0,94 ( $95 \% \mathrm{CI}=0,825$ to 1,000). Serum anti-TPO levels ranged from 28 to $1300 \mathrm{kU} / \mathrm{L}$. Passing \& Bablok regression analysis showed statistically significant systematic, but not proportional differences between the two compared collection tubes (regression equation: $\mathrm{y}=-7,006731+1,000158 x$; Intercept $\mathrm{A}=-7,0067 ; 95 \% \mathrm{CI}=-9,7218$ to $-0,6252 ; \mathrm{Slope} \mathrm{B}=1,0002 ; 95 \%$ $\mathrm{CI}=0,9108$ to 1,0112$)$. Cusum test for linearity showed no significant deviation from linearity $(\mathrm{P}=0,33)$. Weighted kappa coefficient was 0,943 $(95 \% \mathrm{CI}=0,833$ to 1,000$)$.

CONCLUSIONS: Study results support the use of both serum collection tubes for thyroid antibodies determination as they give clinically equivalent results.

Key Words: thyroid autoantibodies, serum, clot activator tube, gel separator tube

\title{
EDTA CONTAMINATION: OLD FOE BUT STILL NOT RESOLVED IN THE LABORATORY
}

\author{
$\underline{\text { R. Marrington }}^{1}$, S. Whitehead ${ }^{2}$, J. French ${ }^{1}$, M. Anderson ${ }^{1}$, F. Mackenzie ${ }^{1}$ \\ ${ }^{1}$ Birmingham Quality [UK NEQAS], University Hospitals Birmingham NHS Foundation Trust, Birmingham \\ ${ }^{2}$ Department of Blood Sciences, New Cross Hospital, Wolverhampton
}

BACKGROUND-AIM: Potassium EDTA (Ethylenediaminetetracetic acid) K2EDTA is an anticoagulant used in blood collection tubes for many laboratory tests. Gross EDTA contamination, from blood sampling collection errors, is easily recognised with marked hyperkalaemia and hypocalcaemia. More subtle contamination is not so easily identified, but can still affect patient management. A case is described where potassium is elevated above the phone limit at $6.51 \mathrm{mmol} / \mathrm{L}$. Adjusted calcium, magnesium and ALP were normal. Repeat bloods showed potassium was $4.2 \mathrm{mmol} / \mathrm{L}$. The first sample contained $>0.5 \mathrm{mmol} / \mathrm{L}$ EDTA. The impact of subtle EDTA contamination on routine clinical chemistry assays has been assessed through the UK NEQAS for Clinical Chemistry EQA Scheme, as well as an audit of the handling of suspected EDTA contamination.

METHODS: Female non-clinical issue donations from NHSBT were pooled, and spiked with 0, 0.25 and 0.5 mmol/L K2EDTA. These three specimens were distributed to 628 participants in the UK NEQAS for Clinical Chemistry scheme at Distribution 1033 (February 2018).

RESULTS: Results were returned by 614 participants, as expected serum potassium was elevated by 0.5 and $1.0 \mathrm{mmol} / \mathrm{L}$, whilst the calcium and magnesium levels were affected by differing amounts dependent on method. Iron, copper and zinc showed method specific issues; however, no significant impact was seen on other analytes within the EQA Scheme including ALP. The participant audit showed a wide range in laboratory practices for identifying EDTA contamination, with 1.2\% participants offering an EDTA service. 32 different protocols were given for which analytes are not reported if EDTA contamination is suspected (from 402 responses).

CONCLUSIONS: EDTA contamination is not a new concept, but despite evidence of a high prevalence in routine clinical laboratories, there is a wide range in practice on handling suspected EDTA contamination, with only a few laboratories routinely measuring EDTA concentrations.

Key Words: EDTA, contamination, EQA, potassium

\section{COMPARISON OF BLOOD COLLECTION TUBES FOR ROUTINE CHEMISTRY ANALYTES}

\author{
$\underline{\text { S. Marevic }}^{1}$, B. Sokolic ${ }^{1}$, R. Laskaj ${ }^{1}$ \\ ${ }^{1}$ University Hospital for Infectious Diseases “Dr. Fran Mihaljevic”, Zagreb, Croatia
}

BACKGROUND-AIM: Serum separator tubes (SST) are not routinely used in our laboratory for venous blood collection. Therefore, the aim of our study was to compare routine chemistry analytes in different serum tubes.

METHODS: Whole blood was taken from 10 volunteers in evacuated tubes: VACUETTE® serum (red cap), VACUETTE® SST (yellow cap) (Greiner Bio-One, Austria) and BD VACUTAINER® trace element tubes (dark blue cap) (Becton-Dickinson, USA). Chemistry analytes were measured on Beckman Coulter (AU680 and AU640). Trace elements cooper and zink were done with atomic absorption (Aanalyst200, Perkin Elmer, USA). Bland Altman analysis was done in MedCalc. The results are shown as difference mean and limits of agreement.

RESULTS: The results for comparison of red and yellow capped tubes in units are as follows: glucose 0,01 $(-0,19-0,21) \mathrm{mmol} / \mathrm{L}$; urea 0,02 $(-0,10-0,14) \mathrm{mmol} / \mathrm{L}$; creatinine $0,10(-1,35-1,55)$ ( mol/L; sodium -0,60 (-2,49-1,29) mmol/L; pottasium -0,02 (-0,39-0,35) $\mathrm{mmol} / \mathrm{L}$; chloride -0,90 $(-3,06-1,26) \mathrm{mmol} / \mathrm{L}$; calcium -0,01 (-0,05-0,03) mmol/L; phosphorus -0,02 (-0,05-0,01) mmol/L; magnesium -0,02 (-0,05-0,00) mmol/L; total bilirubin 0,50 (-0,89-1,89) (mol/L; AST -0,7 (-3,5-2,1) U/L; ALT -0,1 (-2,3-2,1) U/L; GGT 0,3 (-1,0-1,6) U/L; ALP -0,6 (-2,49-1,29)U/L; CK -0,3 (-4,2-3,6) $\mathrm{U} / \mathrm{L}$; LD -9,4 (-34,7-15,9) U/L; AMS -0,5 (-3,3-2,3) U/L; total proteins -0,4 (-2,7-2,0) g/L; albumin -0,81 (-2,21-0,59) g/L; CRP 0,01 (-0,14-0,16) mg/L. Results for tubes capped red and yellow; red and blue; blue and yellow are as follows: iron 0,70 (-0,25-1,65); 0,60 (-0,77-1,97); 0,1 (-1,01-1,21 (mol/L; UIBC 1,00 (-0,85-2,85); 1,20 (-0,82-3,22); -0,20 (-2,00-1,60) \mol/L; cooper 0,22 (-0,37-0,81); 0,33 (-0,25-0,91); -0,11 (-0,73-0,51) (mol/L; zink - $0,70(-2,25-0,85) ;-0,49(-2,55-1,57) ;-0,21(-2,18-1,76) \int \mathrm{mol} / \mathrm{L}$

CONCLUSIONS: SST tubes showed no clinically relevant difference for the measured analytes in normal range and, therefore, can be used in our laboratory for routine chemistry.

Key Words: serum separator tubes, chemistry analytes, trace elements 


\title{
A CHANGE IN COAGULATION TUBES: IMPACT IN SAMPLE QUALITY
}

\author{
$\underline{\text { S. Carrasco }}{ }^{1}$, C. Fernandez ${ }^{1}$, X. Clotet $^{1}$, Y. Rico ${ }^{1}$, L. Diaz ${ }^{1}$, M. Montagut ${ }^{1}$, M. Regue ${ }^{1}$, M. Ruestes ${ }^{1}$, S. Pico ${ }^{1}$, M. Bernal ${ }^{1}$, A. Munoz ${ }^{1}$, A. Criado ${ }^{1}$, \\ M. Ibarz ${ }^{1}$ \\ ${ }^{1}$ Laboratory Medicine Department, University Hospital Arnau de Vilanova, IRBLleida, Lleida, Spain
}

BACKGROUND-AIM: Between December 2017 and March 2018, it was carried out in our phlebotomy centers the replacement of the Vacuette ${ }^{\circledR}$ sodium citrate tube (TV) with the one of Becton Dickinson ${ }^{\circledR}$ (TBD) used for the coagulation tests. One of the most frequent errors in these samples was the rejection of them due to "Insufficient sample" (IS). Therefore, the impact of the tube change was evaluated by means of a comparative study between both, assessing the IS error in each of them as a preanalytic indicator.

METHODS: A comparative study between the two types of tubes was performed. To this end, all the extraction centers with a number of coagulation samples $\geq 50$ were taken into account in the period between 1st of March and 31th of May of the years 2017 (TV) and 2018 (TBD). The preanalytical indicator used was IS which is related to the appropriate blood-anticoagulant ratio. A nonparametric statistical analysis was carried out performing the Kruskal-Wallis $\mathrm{H}$ test using the PSPP software.

RESULTS: The number of total coagulation samples in TV and TBD were 16191 and 16895, respectively. Regarding those samples, the number of IS errors in TV and TBD was 203 and 1, respectively. Attending to the statistical analysis, a p-value of 0.000 was obtained.

CONCLUSIONS: The indicator IS suggest a decrease in the percentage of errors that was verified

with the significance of the statistical study, resulting in a positive change to the TBD. This could be related to the predefined vacuum of each type of tube to achieve the exact collection volume. Therefore the incorrect filling of the tubes affects the correct blood-anticoagulant ratio.

Key Words: preanalytical quality indicators, citrate tube, anticoagulant ratio

\section{VERIFICATION OF MINICOLLECT® Z SERUM TUBES WITH AND WITHOUT GEL}

\author{
$\underline{\text { I. Herceg }}^{2}$, I. Celap ${ }^{3}$, N. Nikolac Gabaj ${ }^{3}$, M. Bozovic ${ }^{3}$, A. Vrtaric ${ }^{3}$, A. Topic ${ }^{3}$, S. Krtanjek ${ }^{1}$ \\ ${ }^{1}$ Department of Oncology and Nuclear Medicine, Medical School University Hospital Sestre Milosrdnice, Zagreb, Croatia \\ ${ }^{2}$ Medical biochemistry laboratory, General hospital "Dr. Tomislav Bardek", Koprivnica, Croatia \\ ${ }^{3}$ University Department of Chemistry, Medical School University Hospital Sestre Milosrdnice, Zagreb, Croatia
}

BACKGROUND-AIM: Minicollect ${ }^{\circledR}$ Z tubes manufacturer has developed a new tube containing same composition, different shape and better features compared to the old one. Therefore, they need to be verified before introduction into routine work.

METHODS: Our study included comparison as follows: 1. qualitative comparison of serum separation ease; 2 . hemolysis degree comparison; 3. comparison of 14 biochemical parameters (ALT, AMY, AST, direct bilirubin, total bilirubin, Ca, CK, Na, K, Cl, CRP, Glc, enzymatic creatinine, LD) and serum indices (H-hemolysis, I-icteria, L-lipemia). First comparison was done between old and new Minicollect ${ }^{\circledR} \mathrm{Z}$ Serum tube (Greiner Bio-One, Kremmsmunster, Austria) $(\mathrm{N}=30)$ and second between new Minicollect ${ }^{\circledR} \mathrm{Z}$ Serum and a new Minicollect ${ }^{\circledR}$ $\mathrm{Z}$ Serum (Separator-with gel) tube $(\mathrm{N}=40)$. Samples were centrifuged at $12100 \mathrm{~g} / 5 \mathrm{~min}$ and analyzed on Abbott Architect c8000 (Abbott Laboratories, Abbott Park, Illinois, USA). Sample was considered hemolysed if H index was $>0.5$. Bland-Altman plots were calculated using Medcalc ${ }^{\circledR}$ v18.10.2 (Ostend, Belgium). Bland-Altman plots derived biases were compared to Westgard's minimum specification for allowable total error.

RESULTS: New tube has an integrated collection scoop and is wider compared to the old one, resulting in easier sampling but lower serum column which makes aliquoting more difficult. However, it's easier to separate serum in gel tube than from the one without gel. Hemolysed samples proportion didn't differ between old (7/30) and new (10/30) tubes without gel, $\mathrm{P}=0.748$, as well between tubes without (9/40) and with gel (16/40), P = 0.148. Proportional bias for AMY (26.3\%; agreement range:-62.8\% to $115.3 \%)$ exceeds Westgard's criteria (21.9\%).

CONCLUSIONS: Study showed that old tube and gel tube are better for serum separation compared to the new tube and tube without gel. AMY activity is higher in old tubes and possible cause should be tested in further investigations. Finally, gel tube is more appropriate for our routine laboratory work.

Key Words: Minicollect ${ }^{\circledR}$ tube, new model, verification

\section{LOCAL VALIDATION OF VACUTEST KIMA (VK) BLOOD COLLECTION TUBES VERSUS BECTON DICKINSON (BD) TUBES}

M.B. Badal Cogul ${ }^{1}$, M.A. Ballesteros Vizoso ${ }^{1}$, P. Argente Del Castillo Rodríguez ${ }^{1}$, L. Valiña Amado ${ }^{1}$, M.I. Pastor García ${ }^{2}$, A.R. Pons Mas ${ }^{2}$, J.M. Bauçà ${ }^{1}$

${ }^{1}$ Department of Laboratory Medicine, Hospital Universitari Son Espases, Palma de Mallorca, Spain

${ }^{2}$ Preanalytical Area of Laboratory Medicine, Hospital Universitari Son Espases, Palma de Mallorca, Spain 
BACKGROUND-AIM: The use of high quality blood collection devices is an aspect of utmost importance where different sample containers may be a source of preanalytical bias, which can impact tests results. The aim of this study was to validate VK blood tubes versus BD tubes following the EFLM WG- PRE recommendations and ISO 15189:2012 preanalytical procedure requirements.

METHODS: We performed single venipunctions to 213 volunteers using BD collection devices (VK compatibility was previously validated by the manufacturer) after a signed informed consent. We validated BD (Gel and clot activator serum $(8.5 \mathrm{~mL}, \mathrm{n}=121), \mathrm{K}_{3} \mathrm{EDTA}$ ( $2 \mathrm{~mL}, 3.6 \mathrm{mg}, \mathrm{n}=121)$, Sodium Citrate $(2.7 \mathrm{~mL}, 0.109 \mathrm{M}, \mathrm{n}=79)$, Trace Elements $(6 \mathrm{~mL}, \mathrm{n}=56)$ ) and VK (Gel and clot activator serum ( $8 \mathrm{~mL}$, $\mathrm{n}=121), \mathrm{K}_{3}$ EDTA (3mL, 5.4mg, $\left.\mathrm{n}=121\right)$, Sodium Citrate $(3.5 \mathrm{~mL}, 0.129 \mathrm{M}, \mathrm{n}=79)$, trace elements $(6 \mathrm{~mL}, \mathrm{n}=56)$ ) tubes. The indicators for the technical validation were evaluated and the acceptable difference for each one was $<1 \%$. For the clinical validation we studied 17 serum, $14 \mathrm{~K}_{3}$ EDTA, 4 sodium citrate and 3 trace element parameters. Validation criteria were: Regression line, Passing Bablok and Difference Plot test.

RESULTS: The difference between VK and BD serum tubes that presented blood contamination was $20.61 \%$ and the difference in trace element tubes that fail to form a vacuum was $1.8 \%$. There were clinical differences in sodium, potassium, chloride, calcium, glucose, lactate dehidrogenase and transferrin (serum), prothrombin time, APTT, fibrinogen (citrate), basophil and monocyte count (EDTA) and zinc (trace elements).

CONCLUSIONS: Tube manufacturers and the pertinent regulatory agency should be informed about the blood contamination of the collection device. It is necessary to modify local reference ranges for parameters in which there is a clinical significant difference or keep using the same ones. Laboratory professionals should check that tubes fulfill the recommended expectations.

Key Words: preanalytical procedure, technical validation, blood contamination, clinical validation

\title{
CITRATE PLASMA QUALITY AS AN ESSENTIAL PART OF CLINICAL LABORATORY HEMOSTASIS TESTING
}

\author{
$\underline{\text { V. Samoylenko }}^{1}$, A. Andreeva ${ }^{2}$, I. Goncharova ${ }^{2}$, A. Amosova ${ }^{2}$, E. Kutueva ${ }^{1}$ \\ ${ }^{1}$ Moscow Region Scientific Institute named after Vladimirsky, Moscow, Russian Federation \\ ${ }^{2}$ Galen company LTD, Moscow, Russian Federation \\ ${ }^{3}$ Company Laboratory project LTD, Moscow, Russian Federation
}

BACKGROUND-AIM: The elapsed time between blood sampling and sample processing has dramatically prolonged due to increasing transportation distance of blood samples after collection and quality of primary citrate tubes may potentially invalidate analysis results. The aim of the present study was to investigate the potential impact of the storage time between blood collection and analysis and identifying the effect of different primary tubes on routine coagulation test results: Activated Partial Thromboplastin Time (aPTT), Prothrombin Time (PT), Fibrinogen (Fb), D-Dimer (DD) and Antithrombin (AT).

METHODS: 4 types of evacuated polymer tubes containing 0,109 M tri-Na citrate were collected from 118 patients including healthy outpatients, pregnant women, and patients on Warfarin sampled in 2 centers. All coagulation tests were performed on ACL TOP analyzer using reagent of the same manufacturer. 4 storage times were investigated: $2 \mathrm{~h}, 4 \mathrm{~h}, 6 \mathrm{~h}$ and $24 \mathrm{~h}$. Statistical analysis was performed by linear regression, the linear correlation coefficient of r-Pearson was calculated for each pair of values at the same storage time. All the tubes were compared with BD Vacutainer ${ }^{\circledR}$ Plus Citrate tube at 0 time.

RESULTS: For all the cases Pearson coefficient demonstrated strong correlation ( $r>0.72)$. PT, Fb, AT and DD $r$ demonstrated very strong correlation for up to 24 hours $(r>0.89)$ in all types of the tubes. Potentially clinically significant time-dependent increase in aPTT could be demonstrated in samples stored more than 4 hours for BD and Sarstedt and 0 hours in other tubes limiting the utilization of these tubes for centralization.

CONCLUSIONS: Obviously introducing of new type of sample collection tubes requires validation process, despite presence of local regulatory certificates.

Key Words: citrate tubes, hemostasis, preanalytics, quality, storage period

\section{DETERMINATION OF PROLACTIN LEVELS IN PSYCHIATRIC PATIENTS -REAL CHALLENGE?}

\author{
$\underline{\text { A. Bakliza }}^{3}$, N. Ruljancic ${ }^{3}$, S. Vuk Pisk ${ }^{1}$, E. Ivezic ${ }^{1}$, K. Matic ${ }^{1}$, N. Lucev $^{1}$, N. Geres ${ }^{1}$, V. Grosic ${ }^{1}$, I. Filipcic ${ }^{2}$ \\ ${ }^{1}$ Department of Integrative Psychiatry, Psychiatric Hospital 'Sveti Ivan' Zagreb, Croatia \\ ${ }^{2}$ Department of Integrative Psychiatry, Psychiatric Hospital 'Sveti Ivan' Zagreb, Croatia, Faculty of Medicine, JJ Strossmayer University of Osijek, \\ Osijek, Croatia \\ ${ }^{3}$ Department of Laboratory Diagnostics, Psychiatric Hospital 'Sveti Ivan' Zagreb, Croatia
}

BACKGROUND-AIM: Blood sampling for determination of prolactin levels in psychiatric patients is challenging job. For fulfilling preanalitical requirements it has to pay attention for patient's preparation and psychiatric therapy. In our study we wanted to investigate association of stress, type of psychiatric therapy and stage of psychiatric disorder with prolactin levels. 
METHODS: 22 patients (16 women, 6 men) were admitted to Psychiatric Hospital 'Sveti Ivan' with psychotic disorders (F20-F29). The mean age was 32,64 with range of 18-55 years. Patient data of psychopharamcotherapy (risperidone, clozapine, olanzapine) and questionnaires scales: DASS21-stress symptoms and PANSS-stage of disease were collected. Blood samples were taken in the morning after 30 min rest. Prolactin, prolactin after precipitation with PEG 6000 and cortisol were measured using clia assay on Beckmann Coulter Access. Statistical analysis was performed with Mann Whitney U test and correlation analysis with Pearson's and Spearman's coefficients using SPSS software and $\mathrm{p} \leq 0.05$ was considered as statically significant.

RESULTS: Our results showed that 16/22 patients had hyperprolactinemia (mean $1100 \mathrm{mIU} / \mathrm{L}$ range =121-3702) and after PEG precipitation prolactin recovery was $<40 \%$ (mean $=8,62 \%$ range $=2,77-33,72)$. Cortisol levels were: mean $336 \mathrm{nmol} / \mathrm{L}$ range $=181,61-457,04)$. There is a significant correlation between PANSS scale (expressed positive, rho $=0,511 \mathrm{p}=0,015$ or negative symptoms, rho $=0,486 \mathrm{p}=0,022$ ) and prolactin. Also there is significant correlation between dose of risperidone( $r h o=0.611 ; \mathrm{p}=0,002)$ and prolactin $(\mathrm{mean} 1370 \mathrm{mIU} / \mathrm{L} \mathrm{range}=725-1798)$. There is no correlation between stress (DASS21) and prolactin, neither between cortisol and prolactin levels $(r=0,241 \mathrm{p}=0,336)$.

CONCLUSIONS: When there is demand for determination prolactin in psychiatric patients it can be expected true hyperprolactinemia specially if risperidone is in therapy and patient has severe stage of psychiatric disorder.

Key Words: prolactin, stress, psychiatric disorder, psychiatric therapy

\title{
DOES THE USE OF VIRAL WITH VACUUM GRIP REDUCE RATES OF CONTAMINATION OF URINARY ROLLECTIONS?: COMPARISON BETWEEN THE USE OF SIMPLE COLLECTING BOTTLES AND COLLECTING BOTTLES WITH VACUUM GRIP
}

\author{
N. Rahmani ${ }^{1,2}$, E. Sebbar ${ }^{1,2}$, S. Rifai ${ }^{1,2}$, S. Lamrabat ${ }^{1,2}$, M. Bensaleh ${ }^{1,2}$, A. Maleb ${ }^{1,2}$ \\ ${ }^{1}$ Microbiology laboratory, the Mohammed VI University Hospital, Oujda, Morocco \\ ${ }^{2}$ Faculty of Medicine and Pharmacy of Oujda, the Mohammed First University, Oujda, Morocco
}

BACKGROUND-AIM: Cytobacteriological examination of the urine (CBEU) is the more often requested examination in the bacteriology laboratory. CBEU remain the key examination of a sure diagnosis of urinary infection. The aim of our work is the view of the uses of collecting bottles with vacuum grip reduces rates of contamination of urinary infections in the microbiology laboratory of Mohamed VI teaching hospital in Oujda.

METHODS: It was a perspective study from the 15/02/2018 to the 15/06/2018 including all the requested cytobacteriological examination of the urine (CBEU) of the ex and inpatients in the Mohammed VI University Hospital of Oujda, during the period from 15/05/2018 to 14/04/2018, the sampling of CBEU from mid-stream urine sample was realized on simple collection bottle. During the period coming from the 15/04/2018 to the 15/06/2018 the sampling of CBEU was realized in collection bottles with vacuum grip. We compared the prevalence of contamination during the two periods.

RESULTS: During the first period of our study we've collected $1126 \mathrm{CBEU}$, the number of contaminated samples was 375, with prevalence of $33 \%$. Within the 2nd period of our study we've collected $1035 \mathrm{CBEU}$; the number of contaminated samples was 362 with a prevalence of $34 \%$. The results of our study prove that there is no significative difference between the use of virals with vacuum grip and simple collecting bottles. In order to ensure the overall reliability of results, the urine collection needs good conditions. If those conditions were not full filled. The CBEU subprimes the conclude to a polymorphic flora.

CONCLUSIONS: The results of our study stressed on the importance of respect of the sampling conditions which are: Careful perineal grooming and seasoning, A mid-stream urine sample, sample collected if possible on morning when waking up analysis on the two hours after collecting. Those conditions don't depend on the nature of collecting bottle (simple or with vacuum grip) according results of our study.

Key Words: cyto-bacteriological urine examination, preanalytical, comparison, contamination, collecting bottle

\section{IMPLEMENTATION OF THE OH/1H HS-CTNT ALGORITHM USING BD VACUTAINER@ BARRICOR TUBES: SIGNIFICANT REDUCTION IN TURNAROUND TIME}

\author{
$\underline{\text { S. Mezger }}^{3}$, Y. Van Cauteren ${ }^{1}$, B. Bekkers ${ }^{2}$, O. Bekers ${ }^{3}$, S. Meex ${ }^{3}$, A. Mingels ${ }^{3}$ \\ ${ }^{1}$ Department of Cardiology and Department of Radiology and Nuclear Medicine, Maastricht University Medical Center, Maastricht, The \\ Netherlands \\ ${ }^{2}$ Department of Cardiology, Maastricht University Medical Center, Maastricht, The Netherlands \\ ${ }^{3}$ Department of Clinical Chemistry, Central Diagnostic Laboratory, Maastricht University Medical Center, The Netherlands
}

BACKGROUND-AIM: To rule-out myocardial infarction, high-sensitivity troponin (hs-cTn) assays are currently recommended by the ESC 2015 guideline together with the $0 \mathrm{~h} / 1 \mathrm{~h}$ algorithm. This requires a very efficient workflow from cardiac emergency (CE) to clinical lab and the use of plasma seems hereby essential. Here, we investigate the implementation of the $0 \mathrm{~h} / 1 \mathrm{~h} \mathrm{hs}$-cTnT algorithm using the new BD Barricor tubes. 
METHODS: Serum (BD Vacutainer ${ }^{\circledR}$ SSTTM, $5 \mathrm{~mL}$ ) and lithium-heparin plasma (BD Vacutainer ${ }^{\circledR}$ BarricorTM, $3.5 \mathrm{~mL}$ ) leftover samples were collected from 49 CE patients at the Maastricht University Medical Center, Maastricht, the Netherlands. Second, all hs-cTnT requests were collected before (07-2014 until 07-2016, $n=9702$, CE $n=1029)$ and after (09-2016 until 09-2018, $n=7866$, CE $n=7601)$ the implementation of the new algorithm. Hs-cTnT (Troponin T hs STAT, Roche Diagnostics) was measured on the Cobas 8000 analyzer (Roche Diagnostics). Turnaround time was estimated as the time between sample registration in the laboratory and release of the result, where all blood samples were send to the lab using the tubing system.

RESULTS: Mean (range) hs-cTnT concentrations were 6.2 (0.5-57.3) ng/L and 6.0 (0.4-57.8) ng/L in serum and plasma, respectively (R 0.995, bias $-1.9 \%, \mathrm{n}=49$ ). In total, hs-cTnT results were released $<60 \mathrm{~min}$ for $77 \%$ of serum versus $94 \%$ of plasma samples, while for CE samples this was $83 \%$ versus $94 \%$. This was true $<45$ min for $53 \%$ versus $78 \%$ and $63 \%$ versus $79 \%$, respectively. Also, median (IQR) turnaround time for hs-cTnT was 43 (35-58) min for serum and 37 (33-43) min for plasma $(\mathrm{P}<0.0001)$. For CE samples, this was 40 (33-52) min and 37 (33-43) min, respectively $(\mathrm{P}<0.0001)$.

CONCLUSIONS: Hs-cTnT concentrations were strongly correlated between serum and lithium-heparin BD Barricor plasma samples. Moreover, the transition from serum to heparin plasma resulted in a significant reduction of hs-cTnT turnaround time with almost all ( $94 \%$ ) results released within 1 hour, thereby fully supporting the $0 \mathrm{~h} / \mathrm{h}$ algorithm.

Key Words: Barricor, troponin, 0/1h ESC algorithm, NSTEMI, turnaround time

\title{
SUITABILITY OF BD VACUTAINER® BARRICORTM BLOOD COLLECTION TUBES FOR ROUTINE ANALYSIS OF 28 CHEMISTRY, 16 IMMUNOASSAY AND 5 HAEMATINICS ASSAYS
}

\author{
$\underline{\text { Z. Maunsell }}^{1}$, E. Siggs ${ }^{1}$, A. Dean ${ }^{1}$, T. James ${ }^{1}$ \\ ${ }^{1}$ Department of Clinical Biochemistry, Oxford University Hospitals NHS Trust, UK
}

BACKGROUND-AIM: The BD Vacutainer ${ }^{\circledR}$ Barricor ${ }^{\mathrm{TM}}$ blood collection tube may provide improved sample quality and could allow consolidation of tests into a single tube, thereby reducing total blood volume collected, total tube numbers, and improving analysis times. This study evaluates the suitability of Barricor ${ }^{\mathrm{TM}}$ for an extended repertoire of Biochemical tests.

METHODS: Blood from 33 healthy volunteers was collected into Barricor, PST II and SST II Advance tubes. An additional Barricor tube was collected from 91 hospital patients. Samples were analysed for 28 chemistry, 16 immunoassay and 5 haematinic assays on the Abbott c16000 and i2000 analysers.

RESULTS: 18/28 Chemistry analytes showed no statistically significant difference between PST II and Barricor. 9/28 had a statistical (p<0.05) but clinically insignificant difference (e.g. creatinine $\mathrm{p}=0.0056, \mathrm{y}=0.99 \mathrm{x}+0.32)$. LDH was the only assay to show both statistical ( $\mathrm{p}=0.0132)$ and potentially clinically significant difference and notable scatter. 8/16 immunoassay analytes showed statistically significant differences between SST II Advance and Barricor. For vitamin D and c-peptide, a small intercept was present. Testosterone and DHEAS showed a $<5 \%$ bias vs serum; FSH, progesterone and insulin biases were all around 10\%. An 8\% negative bias was observed for PTH in K2EDTA vs Barricor. 5/5 haematinics assays showed statistically significant differences between SST II and Barricor. However the bias for all analytes was clinically acceptable at $<5 \%$. CONCLUSIONS: With the exception of LDH, all chemistry analytes showed no clinically significant difference between Barricor and PST II. Haematinics and immunoassays showed more variation between Barricor and SST II, but differences were clinically acceptable. Barricor offers significant advantages over gel tubes and is suitable for routine biochemical testing, including analytes measured by immunoassay for which serum is traditionally the preferred specimen.

Key Words: Barricor consolidation serum

\section{COMPARISON OF BD VACUTAINER® BARRICORTM TUBE WITH THE BD VACUTAINER® PSTTM II TUBE AND BD VACUTAINER® K2EDTA TUBE FOR GENERAL AND SELECTED SPECIAL CHEMISTRY ANALYTES}

$\underline{\text { L. Olivier }}^{1}$, Z. Maunsell ${ }^{1}$, A. Dean ${ }^{1}$, T. James ${ }^{1}$

${ }^{1}$ Department of Clinical Biochemistry, Oxford University Hospitals NHS Trust, UK

BACKGROUND-AIM: BD offers a novel form of blood collection tube, the BD Vacutainer ${ }^{\circledR}$ Barricor ${ }^{\mathrm{TM}}$ tube incorporates a mechanical separator to yield a plasma sample of higher quality and improved stability. The aim of this study is to evaluate how this new tube compares to those already in use (BD PST ${ }^{\text {TM }}$ II or BD K $\mathrm{EDTA}_{2}$ ) for general and selected special chemistry analytes, as well as comparing the stability of analytes measured traditionally in serum.

METHODS: Samples were collected from 20 healthy adult participants into BD Barricor ${ }^{\mathrm{TM}}$ and either BD PST ${ }^{\mathrm{TM}}$ II or BD K_EDTA tubes. For each participant one set of tubes (one Barricor ${ }^{\mathrm{TM}}$ and one PST ${ }^{\mathrm{TM}}{ }_{\mathrm{II}}$ or K $\mathrm{K}_{2} \mathrm{EDTA}$ ) were centrifuged immediately (representing $0 \mathrm{~h}$ ). Further samples were spun at $4 \mathrm{~h}, 8 \mathrm{~h}, 12 \mathrm{~h}$ and $24 \mathrm{~h}$ following collection. Paired samples were tested for the following analytes using Abbott Architect c16000 and i2000 analys-

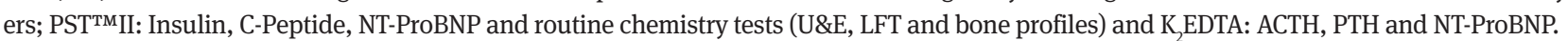


RESULTS: Analytes in each tube type, for each time point were compared against the baseline, and the \% difference calculated. No significant differences were observed between tube types with the exception of: lactate increased by $>50 \%$ for both Barricor ${ }^{\mathrm{TM}}$ and PST ${ }^{\mathrm{TM}} \mathrm{II}$ at $8 \mathrm{~h}$; potassium increased by $>30 \%$ for both Barricor and PST ${ }^{\mathrm{TM}}$ II at $8 \mathrm{~h}$; ACTH increased by $>10 \%$ in Barricor ${ }^{\mathrm{TM}}$ only at $12 \mathrm{~h}$; LDH increased by $>20 \%$ in Barricor $^{\mathrm{TM}}$ at $4 \mathrm{~h}$ and by $>10 \%$ in PST ${ }^{\mathrm{TM}} \mathrm{II}$ at $8 \mathrm{~h}$ and AST increased by $>10 \%$ in Barricor $^{\mathrm{TM}}$ and PST ${ }^{\mathrm{TM}} \mathrm{II}$ at $24 \mathrm{~h}$.

CONCLUSIONS: The stability of the analytes examined is equivalent in Barricor ${ }^{\mathrm{TM}}$ and PST ${ }^{\mathrm{TM}} \mathrm{II}$, with the exception of LDH and AST. ACTH appears to be marginally more stable in $\mathrm{K}_{2}$ EDTA compared to Barricor ${ }^{\mathrm{TM}}$ from 12 hours onwards. Consolidation of tests Barricor ${ }^{\mathrm{TM}}$ is possible for these assays and could have significant advantages for cost, turnaround time and laboratory workflow if adopted.

Key Words: Barricor, stability

\title{
RESPONSIBLE CHOICE: HOW THE SELECTION OF PRE-ANALYTICAL SYSTEMS CAN AFFECT THE FINAL RESULTS OF LABORATORY ANALYSIS. ON THE EXAMPLE OF THE COMPARISON OF DIFFERENT VACUUM SYSTEMS FOR COAGULATION TESTING
}

\author{
$\underline{\text { L. Stankevich }}^{1}$, B. Gorodeckiy ${ }^{1}$ \\ ${ }^{1}$ Gontard \& Cie Group, Moscow, Russian Federation
}

BACKGROUND-AIM: To demonstrate how the pre-analytical system selection can affect efficiency of laboratory diagnostics we compare the performance of sodium citrate evacuated tubes $(2.7 \mathrm{ml}, 3.2 \%)$ of different manufactures on selected coagulation analytes

METHODS: Four different evacuated tubes (BD Vacutainer ${ }^{(B D}$, USA), Univac $\left(\right.$ (Eiliton, Russia), Lind-Vac $\left(\right.$ (Improvacuter ${ }^{\circledR}$, China) and Vacumine ${ }^{(}$(CPMPP Co., China) have been compared using pre-analytical quality criteria such as: 1) fill volume, 2) hemolysis rate, 3) clots and other interference. In addition routine coagulation tests have been performed and compared: 1. thrombin time (TT), 2 . fibrinogen (F), 3. activated partial thromboplastin time (aPTT) and 4. International normalized ratio (INR)

RESULTS: In the first study evacuated blood collection systems demonstrated different performance on fill volume criteria, resulted in rejection rate of $1 \%$ (BD Vacutainer ${ }^{\circledR}$ ), 9\% (Univac ${ }^{\circledR}$ ) and $5 \%$ (Vacumine ${ }^{\circledR}$ ). In the second study, the rejection rate for fill volume comprised of $10 \%$ for Univac $\AA$, 35\% for Lind-Vac and 0\% for BD Vacutainer ${ }^{\circledR}$. Coagulation results of the first study revealed 5,89\% differences between BD and Univac ${ }^{\circledR}$ for aPTT and 6,25\% differences between BD and Vacumine ${ }^{\circledR}$ for INR. Results of the second study demonstrated 7,78\% differences between BD and Univac $\AA$ and 13,09\% differences between BD and Lind-Vac $®$ for TT

CONCLUSIONS: The obtained results show that the accuracy and reproducibility of coagulation results depend not only on pre-analytical practices and standardization of blood sampling procedures, but also on the type and quality of evacuated tubes used.

Key Words: pre-analytical systems, sodium citrate tubes, coagulation, laboratory efficiency

\section{COMPARISON OF URINE DRUG ABUSE TESTING ANALYZED IN GLASS AND PLASTIC TUBES}

\author{
$\underline{\text { R. Nar }}{ }^{1}$, K. Akpınar ${ }^{1}$, S. Demir ${ }^{1}$, T. Toker Uğurlu ${ }^{2}$, E. Avc1 ${ }^{1}$ \\ ${ }^{1}$ Pamukkale University, Faculty of Medicine, Department of Biochemistry, Denizli, Turkey \\ ${ }^{2}$ Pamukkale University, Faculty of Medicine, Department of Psychiatry, Denizli, Turkey
}

BACKGROUND-AIM: Drug abuse tests are applied to determine drug use for the purpose of combating disease, crime, or substance. Laboratory test results are used in medical decisions such as diagnosis, treatment, or follow-up finding. The aim of this study is to investigate whether the plastic tubes can be an alternative to the glass tubes for drugs of abuse analysis in urine.

METHODS: The urine analysis of 40 drug abusers, between 18-65 years old and applied to Psychiatry Clinic of Pamukkale University Hospital, were included in this study. The fresh urine samples were collected in containers and separated in glass and plastic tubes at the same time. The level of amphetamines (amp), benzodiazepines (bnz), cocaine or metabolites (coc), opiates (opi) and cannabinoids (thc) in urine detected on otoanalyzer (Roche Cobas 6000 c702 Modular Analyzer, Mannheim Germany) with photometric method. SPSS22 program was used for statistical analysis.

RESULTS: The medians and minimum-maximum values of amp, bnz, coc, opi and thc in plastic tubes were 66.6 (0-345), 56.5 (0-3460), 16 (0-1332), 51 (0-2103), 4.5 (0-350) respectively. The medians and minimum-maximum values of amp, bnz, coc, opi and thc in glass tubes were 62.5 (0-381), 50.5 (0-3472), 17 (0-1301), 60 (0-2168), 5 (0-350), respectively. There was a significant high correlation between all results for the same analytes measurement in both tubes $(\mathrm{p}=0.0001)$. When the results were evulated as positive and negative according to cut-offs (for positivity: amp $>500 \mu \mathrm{g} / \mathrm{L}, \mathrm{bnz}>300 \mu \mathrm{g} / \mathrm{L}, \mathrm{coc}>150 \mu \mathrm{g} / \mathrm{L}$, opi $>2000 \mu \mathrm{g} / \mathrm{L}$ and thc $>50 \mu \mathrm{g} / \mathrm{L}$ ), the negative results for amp, bnz, coc, opi and thc were 40 (\%100), 37 (\% 92.5), 39 (\%97.5), 39 (\%97.5), 37 (\%92.5), respectively in both tubes.

CONCLUSIONS: According to our study, plastic tubes can be used instead of glass tubes in the analysis of drugs of abuse but more comprehensive studies should be performed with more patients in this subject.

Key Words: urine, collecting tubes, drug abuse testing, substance abuse 


\title{
IMPLEMENTATION OF THE JOINT EFLM-COLABIOCLI RECOMMENDATION FOR VENOUS BLOOD SAMPLING IN CLINICAL HOSPITAL AND LABORATORY - POINT TO TUBE VERIFICATION FOR COAGULATION ANALYSES
}

\author{
$\underline{\text { N. Chokrevska Zografska }}^{1}$, V. Dirjanska-Filipche, D. Stevanoska, S. Rajovski, K. Stojanovska, E. Jovchevska, A. Stojkovski ${ }^{2}$ \\ ${ }^{1} \mathrm{CH}$ Acibadem Sistina, Diagnostic laboratories, Skopje, Macedonia \\ ${ }^{2} \mathrm{CH}$ Acibadem Sistina, Quality department, Skopje, Macedonia
}

BACKGROUND-AIM: Introduction: In a hospital accredited by the JCI standard, and a laboratory accredited by ISO 15189 standard, the center of attention is safety. As soon as the Joint EFLM-COLABIOCLI Recommendation for venous blood sampling were declared, in July 2018, they were implemented it in everyday routine. The recommendations were similar to the existing protocols that were already followed by all the personnel in the hospital and laboratory. A thorough education of all personnel that is involved in venous blood sampling was organized, with the recommended knowledge test. A comparison of two different products for blood collection was performed, to prove that the safety for the patients is not being questioned. The aim is to present the process of tube validation for coagulation analysis as part of implementation of the recommendations for the pre-analytical phase.

METHODS: Methods: The tubes for comparison were BD Lot. No.8130590, date 02-2019 and Greiner, Lot No. A18073RC, date 07.10.2019. The tested analyses were aPTT, PT, Thrombin time, Fibrinogen and D-Dimer. 60 patients were included in the study. Both sample tubes were filled upon collection from the same patient, and the same requested tests were performed on the analyzer, consecutively. The analysis were performed on coagulation analyzer SYSMEX CA 600, reagents from Siemens, QC results were within defined ranges of uncertainty of measurement. RESULTS: Results: After Student's t-test was performed, the results for all analyses showed no statistical difference, which proved that the quality of blood collection tubes had no influence on precision and accuracy of the measured parameters.

CONCLUSIONS: Conclusion: Following of the recommendations for venous blood sampling, as well as following the quality indicators in the pre-analytical phase, is a must in the upgrading of the whole quality control processes in the laboratory.

Key Words: pre-analytical phase, tube validation, coagulation, quality indicators

\section{PILOT STUDY FOR GLUCOSE MEASUREMENT: PLASMA OR SERUM?}

\author{
B. Sánchez - Jacinto ${ }^{1}$, G. Lima - Oliveira ${ }^{2}$, G.C. Guidi ${ }^{2}$ \\ ${ }^{1}$ Section of Blood Bank, Department of Clinical Pathology, Cayetano Heredia Hospital, Lima, Perú \\ ${ }^{2}$ Section of Clinical Biochemistry, Department of Neurosciences, Biomedicine and Movement Sciences, University of Verona, Verona, Italy
}

BACKGROUND-AIM: The measure of blood glucose assay is one of the most frequent laboratory assays, employed for diabetes diagnosis and treatment. Yet, ADA and WHO recommend plasma glucose as specimen of choice since the concentration of glucose in plasma is higher than in whole blood due its higher water content. Several factors influence the stability of glucose values after collection, being in vitro glycolysis the main cause of reduction at a rate of $5 \%$ to $7 \%$ per hour. Accelerated decrease can be observed with increased ambient temperature and in samples with high white blood cell, platelet and erythrocyte counts. The aim of this study was to compare the fasting glucose levels either in plasma or in serum under identical conditions.

METHODS: A cross sectional, retrospective study, included a group of 21 fasting volunteers (14 male and 7 female, mean age $30.14 \pm 9.16$ y.). The blood was collected by a single phlebotomist into two vacuum tubes, as follows: K2EDTA tubes and tubes with clot activator. Both tubes were maintained in upright position for $30 \mathrm{~min}$ at room temperature to allow clot formation. Then, all the tubes were centrifuged at $3500 \mathrm{rpm}$ for $10 \mathrm{~min}$ at room temperature. Glucose was measured in a CONTEC BC 300 semi-auto analyzer with glucose oxidase method. Data were expressed as mean \pm standard deviation and linear regression and Bland Altman plots were performed using STATA 14 software.

RESULTS: The mean glucose concentration was $91.45 \pm 10.38 \mathrm{mg} / \mathrm{dl}$ for plasma and $89.88 \pm 13.20 \mathrm{mg} / \mathrm{dl}$ for serum. Correlation coefficient between plasma glucose and serum glucose was $0.76(\mathrm{R} 2=0.58)$. The mean relative difference between plasma glucose and serum glucose was $+1.74 \%$. Based on linear regression, $1 \mathrm{mg} / \mathrm{dl}$ of mean plasma glucose corresponded to $0.98 \mathrm{mg} / \mathrm{dl}$ mean serum glucose CONCLUSIONS: The results of our research show that the bias between plasma glucose and serum glucose in people with normal cell blood count is small and not significant.

Key Words: glucose, serum, plasma, blood collection tube

\section{SAMPLES FROM DIALYZED PATIENTS: A CHALLENGE FOR THE PROTEIN LABORATORY}

$\underline{\text { C. Quiñones-Torrelo }}^{1}$, M. Díaz-Giménez ${ }^{1}$, Á. Corchón-Peyrallo ${ }^{1}$, A. Pozo-Giráldez ${ }^{1}$, E. Rodríguez-Borja ${ }^{1}$, A. Carratalá-Calvo ${ }^{1}$

${ }^{1}$ Hospital Clínico Universitario de Valencia, Spain

BACKGROUND-AIM: The detection of gammopathies of renal significance can be specially complicated in dialyzed patients due to intrinsic preanalytic interferences mainly related to disorders in coagulation. In this study, we value the impact that this type of samples has in the daily work of the laboratory as well as propose a protocol to avoid unnecessary analysis. 
METHODS: A total of 217 serum samples were analyzed. When abnormalities in the electropherogram were observed, the immunofixation was carried out. If negative, then we repeat the study after sample ethanol treatment to rule out fibrinogen interference. For method validation, the same protocol was applied to a group of lithium heparin plasma samples to calculate the percentage of removed fibrinogen.

RESULTS: Electropherogram was normal for 168 of the samples analyzed. A potentially pathological proteinogram was obtained for 49 of them. From these ones, immunofixation resulted negative in 29 and showed a clear monoclonal protein in gamma area, no related to fibrinogen, for 10 cases. In the electrophoresis of the remaining 10 samples it was found a peak at the fibrinogen location which could be interpreted as a gammopathy. This fact was confirmed by the immunofixation analysis. The gamma globulin/IgG ratio was a reliable tool to improve the screening, mainly in those cases in which proteinogram was not clear even after ethanol precipitation.

Lithium heparin plasma samples treatment showed good quantitative removal of fibrinogen of 92.1\% (9.1), which validates the proposed method.

CONCLUSIONS: According to the results, about $20 \%$ of the serum samples from dialyzed patients can provide abnormal proteinogram suggesting malignancy. However, in $60 \%$ of them no monoclonal bands are detected in the immunofixation, which means an unnecessary waste of time and resources. This can be avoided by means of a previous and effective treatment of the sample that allows discarding the presence of interferences as we reflect in this work.

Key Words: preanalytical phase

\title{
INVESTIGATION OF POTENTIAL TURNAROUND TIME AND QUALITY IMPROVEMENTS WHEN USING BD VACUTAINER@ BARRICOR ${ }^{T M}$ AND CENTRIFUGATION AT SOURCE IN A BUSY EMERGENCY DEPARTMENT.
}

\author{
$\underline{\text { G. Mckeeman }^{1}}{ }^{1}$, L. Mcadam ${ }^{1}$, L. Reilly ${ }^{1}$, F. O'neill ${ }^{1}$, S. Lavery ${ }^{1}$, R. Loughlin ${ }^{1}$, S. Coward ${ }^{1}$ \\ ${ }^{1}$ Royal Victoria Hospital, Belfast Health \& Social Care Trust, Belfast, Northern Ireland
}

BACKGROUND-AIM: Our automated clinical biochemistry laboratory handles approx. 7000 samples per day from primary \& secondary care, including high priority samples from five separate emergency departments. Delivering a faster result turnaround time for acute wards is an on-going challenge. Barricor ${ }^{\mathrm{TM}}$ tubes allow for faster sample separation and a cleaner sample with a mechanical barrier between the plasma and cells. We aimed to examine the use of Barricor ${ }^{\mathrm{TM}}$ tubes and sample preparation within ED before transport to the laboratory to assess if this allowed for faster result reporting.

METHODS: A lab staff member was embedded within the Emergency Department for selected time periods (8am - 8pm; 4pm - midnight) over a 3 week period. Samples were processed at source (LIMS entry and centrifugation at $4000 \mathrm{~g}$ for 3 mins) and sent via the pneumatic chute system to the laboratory. On arrival in the laboratory, samples were taken directly to the analysers (Roche c701) by-passing the busy specimen reception.

RESULTS: Result turnaround times (TAT) were reviewed and compared to similar times when samples were transported (via pneumatic tube) after collection and processed in the laboratory. Overall average total TAT remained similar, with a median of 70 min. However, TAT improvements of up to 15 min were evident on particular days when samples were processed in ED. In addition, there was faster identification of haemolysed and small volume samples, leading to quicker re-bleed times, and a reduction in the amount of urgent samples handled by the lab specimen reception.

CONCLUSIONS: Embedding a lab staff member in ED and processing samples at source has the potential to improve result TAT for general biochemistry testing. Improved communication was possible between ED and the laboratory when the lab member was present, which was welcomed by the ED team. Pre-analytical sample errors were detected faster and handled more efficiently when samples were processed at source.

Key Words: BD Barricor, emergency department, quality, turnaround

\section{HEPARIN AND CITRATE CARRYOVER DURING BLOOD COLLECTION AND THE ORDER OF DRAW.}

\author{
$\underline{\text { M.H. Keppel }}{ }^{1}$, S. Auer ${ }^{1}$, T.K. Felder ${ }^{1}$, H. Oberkofler ${ }^{1}$, C. Mrazek ${ }^{1}$, E. Haschke-Becher ${ }^{1}$, J. Cadamuro ${ }^{1}$ \\ ${ }^{1}$ University Institute for Medical and Chemical Laboratory Diagnostics, Paracelsus Medical University, Salzburg, Austria
}

BACKGROUND-AIM: Evidence on additive carryover risk and carryover volumes in phlebotomy is sparse. We aimed to quantify potential carryover of citrate and heparin and volumes needed to alter clinical chemistry and coagulation parameters.

METHODS: We simulated standardized and non-standardized phlebotomies to quantify the risk of carryover of sodium-citrate and lithiumheparin additive in distilled water using sodium and lithium measurements as surrogates. Then, we investigated effects of increasing volumes of pooled citrated-heparinized blood on sodium, potassium, chloride, magnesium, total and ionized calcium, phosphate and LDH in pooled heparinized-only blood samples. Likewise, we investigated effects of increasing volumes of pure (tri-sodium) citrate on parameters in pooled 
heparinized-only blood samples. Also, we investigated effects of increasing volumes of pooled citrated-heparinized-whole blood on heparin (anti-Xa) activity, lithium concentrations, activated partial thromboplastin time (aPTT), prothrombin time (PT), and thrombin time (TT) in pooled citrated-only-whole blood samples. We interpreted significance according to reference change values (RCV) in Welch-Satterthwaite t-tests. Clinical chemistry and coagulation parameters were measured on a Roche COBAS 8000 and a Siemens BCS XP System.

RESULTS: Standardized and non-standardized phlebotomy simulations revealed no significant differences in concentrations of surrogate markers. Except for ionized calcium, which was significantly altered at $50 \mu \mathrm{L}$, we found no significant inferences on clinical chemistry parameters up to $100 \mu \mathrm{L}$ citrated blood carryover. Our investigations of pure citrate carryover revealed similar results. Heparinized blood carryover showed no significant interference on aPTT and TT up to $5 \mu \mathrm{L}$ and on PT up to $50 \mu \mathrm{L}$

CONCLUSIONS: Our results suggest that during a standardized phlebotomy, heparin or citrate contamination is highly unlikely. Furthermore, actual carryover volumes are likely beyond adverse carryover volumes

Key Words: order of draw, carryover, citrate, heparin, reference change values

\title{
COMPLIANCE WITH VENIPUNCTURE STANDARDS: AN EXAMPLE.
}

\author{
I. Garcia Del Pino $^{1}$, P. Rodriguez Vazquez ${ }^{2}$ \\ ${ }^{1}$ Area Laboratory / University Hospital Complex A Coruna, A Coruna, Spain \\ ${ }^{2}$ Area Laboratory, University Hospital Complex A Coruna, A Coruna, Spain
}

BACKGROUND-AIM: The blood testing quality depends to a large extent on sample quality and the blood sample depends mainly on phlebotomy procedure. This process occurs sometimes outside the laboratory's direct supervision and it is performed by nursing staff whose training or skills are unknown.The aim of this study is to verify the weaknesses of the phlebotomy procedure in several facilities that send their samples to our laboratory. As ultimate goal, this will allow us to know in which issues a better training of the collector staff should be implemented.

METHODS: An observational study was conducted in May-June 2016 in 18 facilities attending outpatients. A checklist of 29 questions was used. Ten of them that focused on key steps of phlebotomy process were selected. The associated risks were evaluated according to the study carried out by the EFLM Working Group on the Preanalytical Phase (Clin Chem Lab Med 2015; 53(9):1321-3).

RESULTS: The incorrect performance percentages and the corresponding associated risk rate were: properly identified request: $0 \%$ with associated risk 12; patient identification according to guidelines: $67 \%$, risk 15; the collector has sanitized his hands correctly: $83 \%$, risk 8 ; verification of the patient correct preparation: $78 \%$, risk 12; cleaning of venopunction site: $61 \%$, risk 9; respect the correct order of draw: $50 \%$ risk 4; correct mixing of tubes: $83 \%$ risk 12; immediate activation of the needle safety system: $0 \%$ risk 8 ; labelling of tubes after venopunction: $28 \%$ risk 20 ; labelling of tubes in front of the patient: $28 \%$ risk 20.

CONCLUSIONS: Observing the level of incorrect performance and the associated risk rate, it is a priority to train the collectors on the correct patient identification and labelling of samples. Next, the collector staff needs training in correct mixing of samples after draw and verification of the patient's preparation. Overall, a better training of collector staff on phlebotomy process is required.

Key Words: phlebotomy, blood testing, standards compliance

\section{FILLING ACCURACY AND PRECISION OF NA-CITRATE COAGULATION TUBES FOR THREE MAJOR BLOOD TUBE MANUFACTURERS}

\author{
A. Kralj ${ }^{2}$, G.L. Salvagno ${ }^{1}$, I. Kuktić ${ }^{2}$, M. Gelati ${ }^{1}$, V. Radišić Biljak ${ }^{2}$, G. Lippi ${ }^{1}$, AM. Šimundić ${ }^{2}$ \\ ${ }^{1}$ Section of Clinical Biochemistry, University of Verona, Verona, Italy \\ ${ }^{2}$ University Hospital „Sveti Duh“, Department of Medical Laboratory Diagnostics, Zagreb, Croatia
}

BACKGROUND-AIM: CLSI H21-A5 guideline (Collection, Transport, and Processing of Blood Specimens for Testing Plasma-Based Coagulation Assays and Molecular Hemostasis Assays) recommends $90 \%$ fill volume for Na-citrate tubes. Tube underfilling or overfilling, greater than $\pm 10 \%$, may lead to false results for some coagulation assays. The aim of this study was to examine the filling accuracy and precision for Greiner, Kima and BD, 3.2\% Na-citrate coagulation tubes.

METHODS: To asses between-lot variations, 3 different lots (20 tubes per lot) of citrate blood tubes from three major manufacturers (Becton, Dickinson and Company (BD), USA; Greiner Bio-One GmbH,Germany; Vacutest KIMA, Italy) were examined. One additional lot from each manufacturer was measured in triplicate (three series of 20 tubes), to assess within-lot variations. All tubes were first weighed empty and then filled with water using $50 \mathrm{~mL}$ syringe and 19G straight needle (ideal filling conditions). Filled tubes were weighed again, in duplicate. For each series of 20 tubes, mean bias (deviation from the ideal tube filling volume) and CV(\%) was calculated.

RESULTS: All biases were within $\pm 10 \%$. Within-lot and between-lots variations in filling volume for all three tube manufacturers was acceptable $(0.4$ to $2.4 \%)$. Greiner tubes were most accurate with biases ranging from -1.0 to $-2.4 \%$. KIMA tubes were least accurate, with a negative bias across all tested lots, ranging from -6.0 to $-7.9 \%$. 
CONCLUSIONS: Although the coagulation test tubes filling accuracy was within $\pm 10 \%$, for all three tested manufacturers, it should be noted that tube filling accuracy may differ between tube manufacturers. Our experiment shows that tube filling may not be ideal even under ideal filling conditions. This leaves little room for patient related tube filling problems.

Key Words: preanalytical phase, coagulation, blood tubes, filling accuracy

\title{
PREANALYTICAL ERRORS IN PERYPHERAL BLOOD COLLECTION FOR HEMATOLOGY TESTS
}

\author{
$\underline{\text { C. Lemos }}^{1}$, I. Gonçalves ${ }^{1}$, V. Muzyka ${ }^{1}$, C. Vaz Carneiro ${ }^{1}$
}

${ }^{1}$ Clinical Pathology Department, CHLN, Lisbon, Portugal

BACKGROUND-AIM: Preanalytical errors (PE) account for 60\%-70\% of errors occurring in the laboratory, most of them occurring during sample collection. When it is made without laboratory supervision, compliance is usually low, owing to high personnel turnover rates, lack of understanding about good laboratory practices, and inadequate training. The aim of this study was to evaluate PE in peripheral blood collection (PBC) when it is performed by laboratory phlebotomists (LP) in comparison with other healthcare professionals (OHP) - doctors and nurses.

METHODS: We verified the number of blood samples collected for hematology tests in our hospital wards between October 2016 and September 2018. Until October 2017, blood samples were collected by LP. From October 2017 to September 2018, PBSC was performed by OHP (doctors and nurses). We compared the number of rejected samples during both periods and the PE involved. We evaluated those results through comparison of proportions and Chi-square.

RESULTS: From October 2016 to September 2017, LP performed 128.981 PBC. From October 2017 to September 2018,113.461 samples were collected by OHP. Rejection rate during the two periods was 1,5\% vs. 2,1\% ( $\mathrm{p}<0.001)$. The most common PE was sample clotting: 1461 (1.1\%) vs. $1801(1,6 \%)(p<0.001)$. Less frequent PE were: excessive or insufficient sample volume, innapropriate collection tube, duplicated requests, hemodiluted sample and incorrect identification.

CONCLUSIONS: Perypheral blood samples collected by LP for hematology tests presented a lower rejection rate in comparison to OHP. Implementation of regular education programs and training could contribute to PE reduction, in order to increase sample quality.

Key Words: preanalytical phase, phlebotomy, errors in laboratory medicine, hematology tests

\section{COLORS OF BLOOD COLLECTION TUBE CLOSURES: A ROOT CAUSE OF INAPPROPRIATE SAMPLE COLLECTION}

\author{
L.M. Brennan-Bourdon $^{2,8}$, E. Aranda ${ }^{5,8}$, M.E. Arredondo ${ }^{1,8}$, S. Flores ${ }^{7,8}$, P. Ochoa ${ }^{4,8}$, B. Varela ${ }^{6,8}$, G. Lima-Oliveira ${ }^{3,8}$ \\ ${ }^{1}$ BIONET S.A., Santiago, Chile \\ ${ }^{2}$ Clinical Laboratory Network from the state of Jalisco, Public Health State Laboratory, Mexico \\ ${ }^{3}$ Department of Neurosciences, Biomedicine and Movement Sciences, University of Verona, Italy \\ ${ }^{4}$ Facultad de Medicina Universidad Católica de Cuenca, Ecuador \\ ${ }^{5}$ Laboratory of Thrombosis and Hemostasis, Pontificia Universidad Católica de Chile, Chile \\ ${ }^{6}$ LAC, Montevideo, Uruguay \\ ${ }^{7}$ Universidad Peruana Cayetano Heredia, Lima, Perú \\ ${ }^{8}$ Latin American Working Group for Preanalytical Phase (WG-PRE-LATAM) of the Latin America Confederation of Clinical Biochemistry (COLA- \\ BIOCLI), Montevideo, Uruguay
}

BACKGROUND-AIM: This case report describes a preanalytical nonconformity due to primary blood tube misclassification by the color of tube closures.

METHODS: Case report: Blood collections from 25 inpatients were performed by two nurses (Nurse A, and Nurse B). Samples were processed using the Cobas $\mathrm{p} 512 \AA$ (Roche), a fully automated preanalytical system based on a computer-controlled stand-alone device for sorting barcoded tubes with the following components: i) input sorter with sorting unit, ii) tube transport, iii) camera module with integrated barcode scanner for sample recognition, iv) decapping module, v) output sorter with sorting unit, vi) control unit, and vii) cap recognition for tubetype identification by a camera system. In our laboratory, this system is connected to an automatic centrifuge unit (Cobas p471, Roche). After centrifugation and automated decapping, all primary blood tubes were placed in dedicated-racks and loaded onto the Cobas 6000 (Roche) for testing.

RESULTS: 16 samples were properly collected by Nurse A, whereas Nurse B collected 9 samples for clinical chemistry testing using evacuated blood tubes containing buffered sodium citrate - tube closure color: translucent light blue - instead of lithium heparin and gel separator - tube closure color: light green. Laboratory staff detected this nonconformity during the results validation process; then, Nurse B was immediately requested to re-collect blood samples from all 9 patients using properly evacuated tubes for clinical chemistry testing. 
CONCLUSIONS: Nurse B recognized primary tubes by the color of the tube closures, not by the additives properly described at tube-label. This daily practice represents a preanalytical nonconformity. Moreover, the preanalytical automation system was not able to distinguish colors (translucent light blue vs. light green). It is time for harmonization of color coding for blood collection tube closures in order to reduce the risk of preanalytical nonconformities and improve patient safety.

Key Words: blood specimen collection, medical errors, patient safety, phlebotomy, specimen handling

\title{
EVALUATION OF VACUETTE® CAT SERUM FAST SEPARATOR BLOOD COLLECTION TUBE FOR ROUTINE CHEMISTRY ASSAYS
}

\author{
$\underline{\text { S. Griebenow }}^{1}$ \\ ${ }^{1}$ Greiner Bio-One Kremsmünster, Austria
}

BACKGROUND-AIM: Clotting time is an important factor in the work flow of blood collection tubes for routine chemistry testing from serum. The key target of VACUETTE $®$ CAT Serum Fast tubes consists in the faster processing time from blood collection to result availability. The faster clotting time provides a clotted sample by the time the sample reaches the laboratory and allows for immediate testing.

METHODS: The study was conducted to show the performance of VACUETTE CAT Serum Fast tubes for routine chemistry analysis up to $48 \mathrm{~h}$ in comparison to VACUETTE Serum Separator tubes. Venous blood was drawn from 50 donors after giving IC into two tubes. Tubes without thrombin were spun after $30 \mathrm{~min}$, and the tubes with thrombin were spun after 5 min clotting time for $10 \mathrm{~min}$ at $1800 \mathrm{~g}$. Initial values were determined on an AU680 and DxI800 (Beckman Coulter). All samples were stored upright at 4-8 ${ }^{\circ} \mathrm{C}$ for replicate testing after $24 \mathrm{~h}$ and $48 \mathrm{~h}$. Comparison analysis was performed at all time points. Clinical evaluation was based on CLIA (Allowable Total Error Table by Data Innovations).

RESULTS: Equivalency for CAT Serum Fast tubes to Serum Separator tubes was shown for routine chemistry assays on Beckman Coulter for healthy donors. Provided a completely clotted sample and clear serum specimens, no significant deviations were found for initial values as well as at $48 \mathrm{~h}$ for 37 biochemical assays tested in both tubes. In agreement to literature, slight systematical deviations in the thrombin tubes were found for some assays such as $\mathrm{Na}, \mathrm{K}, \mathrm{Cl}$, and Gluc due to the faster coagulation process. Stability over $48 \mathrm{~h}$ was shown for all assays except Troponin I (6h).

CONCLUSIONS: The thrombin tube gave comparable test results to current serum separator tubes for most common biochemical assays in clinical laboratories. The tube containing thrombin provides rapid turnaround times in the laboratory by shortening the clotting time, providing accurate testing results and being suitable for emergency testing.

Key Words: fast TAT, biochemistry, emergency testing

\section{TEST ASSESSMENT OF VITROS ECIQ IMMUNODIAGNOSTIC ANALYSER IN DETECTION OF MACROPROLACTIN}

\author{
$\underline{\text { E. Arnaut }}^{1}$ \\ ${ }^{1}$ Department of medical-biochemical and immunological diagnostics Cantonal Hospital Zenica, Bosnia and Hercegovina
}

BACKGROUND-AIM: To investigate whether or not additional pretreatment in samples of serum with poly ethylene glycol(PEG) could improve accuracy of PRL(prolactin) laboratory results in Vitros ECiQ analyser in compare with symptoms,condition associated with high PRL level and MRI imaging.The presence of M-PRL(makroprolaktin) is a significant cause of elevated PRL in all automated systems. The current convention is to report results as a percentage of total PRL recovered after PEG treatment $>40 \%$ macroprolactinemia is rule out.

METHODS: The study included samples of 35 women and one sample of external quality control with high or previous history of high level serum PRL.The analysis of PRL were performed on Vitros ECiQ Immunodiagnostic analyser according to accepted protocols of the International Federation of Clinical Chemistry and Laboratory Medicine (IFCC).In order to precipitate M-PRL was used 25\% PEG.

RESULTS: Serum PRL level after direct measurement was considerably higher 566,5mIU/L (412-946,75) than after PEG treatment 413 mIU/ $\mathrm{L}(331,25-659,75)$ with statistical significance $\mathrm{p}<0,001$. Analysing the comparative difference between the directly and PEG treated serum PRL was not statistically significant difference between the patients stratified by the state / diagnosis $(\mathrm{p}=0.336)$.

CONCLUSIONS: $88,9 \%$ of PRL results were recovery $>40 \%$ in conditions associated with high level serum PRL.11,1\% of PRL results were recovery $<40 \%$ in cases where the expected cause or symptoms of high PRL is rule out. PEG precipitation serum samples should be done in order to avoid unnecessary diagnostic,misdiagnosis and inappropriate treatment especially in asymptomatic patients with high level serum PRL results unknown causes.

Key Words: hiperprolactinemia, macroprolactin, PEG, recovery 


\title{
HEPARIN ANTICOAGULATION AS A PREANALYTICAL INFLUENCING FACTOR ON THE ASSESSMENT OF BLOOD CELL-DERIVED BIOMARKERS
}

\author{
$\underline{\text { R. Huber }}{ }^{1}$, D. Küper ${ }^{1}$, R. Attili ${ }^{1}$, B. Lüns ${ }^{1}$, K. Brand ${ }^{1}$, K. Weissenborn ${ }^{2}$, R. Lichtinghagen ${ }^{1}$ \\ ${ }^{1}$ Hannover Medical School, Institute of Clinical Chemistry, Hannover, Germany \\ ${ }^{2}$ Hannover Medical School, Department of Neurology, Hannover, Germany
}

BACKGROUND-AIM: During blood sampling anticoagulants alter biomarker expression differentially and thus may represent an influencing factor in the preanalytical phase for the validity of distinct measurants. We aimed to evaluate the effects of different anticoagulants on biomarker regulation using matrix metalloproteinase (MMP)-9 as an example for cytokine-regulated gene expression in blood cells.

METHODS: MMP-9 and cytokine expression was determined (ELISA, qPCR, Proteome Profiler Array) in dose-/time-dependent experiments. Anticoagulants (HMWH, LMWH, EDTA, citrate) and known contaminants (over sulfated chondroitin sulfate (OSCS)) were applied in individual, double, and triple co-culture experiments using monocytic THP-1, Jurkat T-, and HT B-cells ( $\mathrm{n}=3$ each).

RESULTS: Anticoagulant incubation has no direct influence on MMP-9 expression of monocytic, T-, or B-cell lines. Treatment with HMWH up to $24 \mathrm{~h}$ significantly increased MMP-9 expression in co-culture experiments of all three cell lines (mRNA: 7.1-fold, $\mathrm{p} \leq 0.01$; protein: 3.3-fold, $\mathrm{p} \leq 0.05$ ) or monocytes/T-cells only (mRNA: 7.9-fold, $\mathrm{p} \leq 0.05$; protein: 3.3-fold, $\mathrm{p} \leq 0.05$ ) whereas LMWH, citrate, and EDTA did not. Supernatant of T-cells incubated with HMWH also significantly induced MMP-9 in monocytes (mRNA: 8.8-fold, $p \leq 0.05$ ). T-cell cytokine profiling revealed that IL 16/Serpin E1 are specifically secreted in response to HMWH. Monocytes stimulated with IL 16/Serpin E1 showed elevated IL-8 expression (mRNA: 5.6-fold, $\mathrm{p} \leq 0.05$ ). T-cell derived IL-16, Serpin E1, and (autocrine) IL 8 induced monocytic MMP-9 expression (mRNA: 7.0-fold, $\mathrm{p} \leq 0.05$ ). In addition, the known HMWH contaminant OSCS massively increased MMP-9 expression by monocytes co-cultured with T-cells (mRNA: approx. 700-fold, $\mathrm{p} \leq 0.05$ ).

CONCLUSIONS: Our results indicate that monocytes show increased production of biomarkers in response to T-cell-derived soluble mediators, which are secreted in the presence of HMWH and known contaminants during blood sampling.

Key Words: blood sampling, anticoagulants, biomarkers, OCSC, cytokines

\section{FOLLICULAR PHASE SAMPLING FOR ANTI-MÜLLERIAN HORMONE (AMH) MEASUREMENTS - A REAL NECESSITY?}

\author{
$\underline{\text { A. Dojder }}^{1}$, M. Tomas ${ }^{1}$, V. Radišić Biljak ${ }^{1}$, AM. Šimundić ${ }^{1}$ \\ ${ }^{1}$ Department of Medical Laboratory Diagnostics, University Hospital “Sveti Duh”, Zagreb, Croatia
}

BACKGROUND-AIM: According to the manufacturer (Beckman Coulter, USA), blood sampling for AMH measurement should be performed between the 2nd and the 4th day of the menstrual cycle. The aim of this study was to assess the possible differences in AMH concentration, relative to the phase of menstrual cycle.

METHODS: Blood samples were collected from 12 healthy volunteers at three phases of regular menstrual cycle (i.e. 3 samples per each subject): 1) between the 2nd and 4th day (follicular phase), 2) on 14th (ovulation) and 3) on 21st day (luteal phase) of menstrual cycle. The samples were analyzed by chemiluminescent method on DXI 600 (Beckman Coulter, USA) analyzer. The concentrations of AMH were compared by non-parametric Friedman test using MedCalc Statistical Software version 16.2.0 (MedCalc Software bvba, Ostend, Belgium), wherein P-value $<0.05$ was considered as significant. Method CV(\%) was assessed in accordance to CLSI EP15-A3 guideline. Mean bias of all subjects for luteal phase and ovulation compared with follicular phase were calculated. According to Bungum et al. (Front Endocrinol, 2018) bias was considered acceptable if $<3.9 \mathrm{pmol} / \mathrm{L}$.

RESULTS: The observed precision was below 5\%. Median age of the participants was 37 years (24-45). Median values (in pmol/L) across menstrual phases were as follows: 16.4 (8.9-28.55) for follicular phase, 20.35 (11.75-28.55) and 18.55 (8.9-26.4) for ovulatory and luteal phase, respectively. Friedman test revealed no statistically significant difference between the median AMH values across the different stages of the menstrual cycle $(\mathrm{P}=0.058)$. Mean biases for ovulation $(-1.7,95 \% \mathrm{Cl}$ : -3.1 - - 0.4$)$ and luteal phase $(0.2,95 \% \mathrm{Cl}$ : -3.5 - 3.9) were within acceptance criteria.

CONCLUSIONS: These preliminary results showed no significant difference of AMH concentration analyzed during different phases of menstrual cycle. Further investigations with higher number of samples should be done to confirm our findings.

Key Words: preanalytical phase, anti- Müllerian hormone (AMH), menstrual phases

\section{HEMATOCRIT INTERFERENCE IN COAGULATION TESTS: WHAT ELSE CAN WE DO?}

$\underline{\text { N. Del Amo }}{ }^{1}$, M. Caballero ${ }^{1}$, L.M. Cruz ${ }^{1}$, M. Dolade ${ }^{1}$, M. Duque ${ }^{1}$, S. Fornies ${ }^{1}$, I. Llovet $^{1}$, M.E. Marquez ${ }^{1}$, F.J. Merida ${ }^{1}$, E.E. Moreno ${ }^{1}$, J. Prieto ${ }^{1}$, M. Ibarz ${ }^{1}$

${ }^{1}$ Spanish Society of Laboratory Medicine (SEQC)-Patient Safety Commission, Spain 
BACKGROUND-AIM: The proper citrate to blood ratio in vacuum tubes should be 1:9, so recommendation for an accurate clot time result is to adjust citrate concentration for blood coagulation specimens with high hematocrit values. High hematocrit is a little considered preanalytical variable and many factors may avoid the correct risk identification. Our objective is to identify the main causes of the failure and the implementation of the corresponding corrective actions

METHODS: Cause and effect analysis (Ishakawa method) was used to identify the main causes of this event.

RESULTS: Ishakawa method identified factors contributing to the effect related to the organization (different people performing or checking out hematological and coagulation tests or time lapsed revision for coagulation and hematological test), to the person (lack of staff education) or the environment (work overload). Corrective actions were guided to: Enhance the detection capability of this risk: A laboratory informatic system aid algorithm was developed and an automatic internal notification is displayed in the informatic laboratory system for the condition hematocrit $>55 \%$ and coagulation test are demanded. And supplement the lab staff training: A specific instruction for the high hematocrit patient management was draw up and informative sessions were programmed for lab staff. The document included details about sample drawing and a citrate correction related to hematocrit chart.

CONCLUSIONS: Successful results have been obtained since no more coagulation test results possible interfered have been released from our lab and patient safety has not been compromised. Staff from different departments are aware of the preanalytical incidence thanks to the internal notification and the instruction has improved staff knowledge and highlighted the importance of the preanalytical incidence.

Key Words: interference, coagulation test, citrate adjustment, risk

\title{
THE POOR QUALITY OF CADAVERIC CORNEA SPECIMENS = A LOT OF FALSELY POSITIVE RESULTS IN SEROLOGICAL TESTING
}

\author{
M. Miletić ${ }^{1}$, D. Grubešic ${ }^{1}$ \\ ${ }^{1}$ Department for Blood Borne Diseases Diagnosis, Croatian Institute of Transfusion Medicine, Zagreb, Croatia
}

BACKGROUND-AIM: Serological testing of cadaveric cornea donors in Croatia includes HBsAg, anti-HCV, anti-HIV-1/2, anti-TP and anti-HBc (if positive, anti-HBs is performed). Cadaveric specimens are collected at variable times after death, often of poor quality (haemolysed or autolyzed) and frequently yield falsely positive results in serological testing. Although Abbott Architect assays (HBsAg Qualitative II, Anti$\mathrm{HCV}$, HIV Ag/Ab Combo, Syphilis TP and Anti-HBc II) are validated for cadaveric specimens, we found a lot of unspecific reactions and implemented confirmatory testing algorithms.

METHODS: From 2016 till October 2018, we got 643 different cadaveric specimens, from the Eye Bank - University Hospital Centre Zagreb. Initial tests used are named Abbott Architect tests. The following tests were used to decide on false or correct positive results: for HBsAg: Bio-Rad Monolisa HBsAg Ultra, Architect Confirmatory test, Vidas HBsAg ULTRA; for anti-HCV: Vidas Anti-HCV, Bio-Rad Monolisa HCV Ag-Ab Ultra v2 Combo, immunoblot (Mikrogen and/or Fujirebio); for HIV Ag/Ab: Dia.Pro HIV Ab\&Ag, Vidas HIV DUO ULTRA, immunoblot (Mikrogen and/or Fujirebio), Vidas HIV P24; for anti-TP: Dia.Pro Syphilis Ab v. ULTRA, TPHA, RPR, immunoblot ((Mikrogen and/or Fujirebio), Dia.Pro Syphilis IgM; for anti-HBc: Dia.Pro HBcAb and Vidas Anti-HBc Total II.

RESULTS: The total falsely positives of Architect tests were found in 3\% (19/634) of HBsAg, 0.3\% (2/634) of anti-HCV, 4.7\% (30/634) of HIV Ag/ $\mathrm{Ab}, 0.3 \%$ (2/634) of anti-TP and $0.6 \%$ (4/634) of anti-HBc results.

CONCLUSIONS: The observed specificity data of the Architect tests used for the initial cadaveric specimen testing show that the highest proportion of falsely positive results is in HIV Ag/Ab test, then HBsAg, anti-HBc and anti-TP/anti-HCV, respectively. Implemented confirmation testing algorithms saved in given period the total of 57 corneas from needlessly discard and assure the supply and safety of corneas for transplantation.

Key Words: cadaveric cornea donors, serological testing

\section{THE DIET, A PREANALYTICAL FACTOR AFFECTING THE METABOLITE PATTERN OF FIRST AND SECOND MORNING URINE}

\author{
$\underline{\text { R. Lehmann }}^{2}$, X. Liu ${ }^{1}$, G. Xu ${ }^{1}$ \\ ${ }^{1}$ CAS Key Laboratory of Separation Science for Analytical Chemistry, Dalian Institute of Chemical Physics, Chinese Academy of Sciences, 16023 \\ Dalian, China \\ ${ }^{2}$ Division of Clinical Chemistry and Pathobiochemistry (Central Laboratory), University Hospital Tuebingen, D-72076 Tuebingen, Germany
}

BACKGROUND-AIM: Non-targeted metabolomics is one of the current key analytical approaches applied to discover novel diagnostic biomarkers. Although urine is a valuable diagnostic sample material it is less common used in metabolomics-driven biomarker discovery studies and no preanalytical recommendation concerning the urine sample material of choice is available up to know. Since morning urine (MU) is the most common specimen we aimed to figure out if first or second MU is more appropriate and should therefore be recommended.

METHODS: First and second MU samples from 35 healthy young subjects were investigated by UHPLC-MS based non-targeted metabolomics. Additionally, urine samples collected after a short dietary intervention (20h before sample collection) of only fast food (FFD, $\mathrm{n}=17$ ) or only vegetarian food $(\mathrm{VF}, \mathrm{n}=18)$ and the metabolite fingerprints were compared. 
RESULTS: Principle component analysis revealed clear differences in the metabolite fingerprints between 1st and 2nd MU. The diet before sample collection grewatly affects the metabolite profile even in 2nd MU. Organic acids, amino acids as well as various acylcarnitines were affected by the diet.

CONCLUSIONS: We recommend 2nd morning urine as sample material of choice for metabolomics-driven biomarker profiling studies. Care should be taken entitling organic acids, amino acids as well as various acylcarnitines as diagnostic biomarkers in urine, since the diet before sample collection may affect the concentration of these metabolites.

Key Words: first morning urine, second morning urine, meal effects, biomarker studies, misleading results, metabolomics

\title{
EVALUATION OF TURNAROUND TIME OF BARRICORTM VS. LITHIUM HEPARIN TUBES FOR TROPONIN I ASSAY IN THE EMERGENCY CLINIC BEFORE AND AFTER THE IMPLEMENTATION OF THE BARRICOR TUBE USE
}

\author{
$\underline{\text { F. Taneli }}^{1}$, C. Ulman ${ }^{1}$ \\ ${ }^{1}$ Dept. of Medical Biochemistry, Manisa Celal Bayar University, Faculty of Medicine, Manisa, Turkey
}

BACKGROUND-AIM: Obtaining reliable results is crucial for laboratory medicine in the first 30 minutes for the emergency care unit unaffected by preanalytical factors. The aim of the present study is to evaluate the turnaround time (TAT) for troponin I assay for Barricor ${ }^{\mathrm{TM}}$ plasma tubes with mechanical separator vs standard Vacutainer ${ }^{\circledR}$ lithium heparin tubes in the emergency unit.

METHODS: We have been using standard Vacutainer ${ }^{\circledR}$ lithium heparin tubes in the emergency care unit for more than a year. Recently, we implemented the use of Becton Dickonson BD Barricor ${ }^{\mathrm{TM}}$ lityum heparin plasma tubes with mechanical separator to obtain better sample quality for troponin I assay. The use of the new tubes started with educating the emergency unit staff including nurses, phlebotomists and doctors. Barricor tube has a 3 minutes 4000xg centrifugation need instead of 10 minutes 2000xg for the Vacutainer ${ }^{\circledR}$ lithium heparin tubes. We compared the TAT before and after the implementation of the new tube for a period of 7 days. The data was obtained from the Laboratory Automation system. We defined TAT as the time the tube arrives to the lab until the result is reported. The high sensitivity (hs) troponin I test was assessed on Beckman Coulter UniCel DXI800 Access immunoassay analyzer.

RESULTS: The study groups were defined as Group 1: TAT with Vacutainer ${ }^{\circledR}$ lithium heparin tubes $(\mathrm{n}=233)$, Group 2: TAT with Barricor ${ }^{\mathrm{TM}}$ lityum heparin plasma $(n=218)$ tubes. The TAT values with mean \pm SD minutes were $44.13 \pm 23.25$ and $35.49 \pm 15.44$ respectively. The target TAT value of 60 minutes was achieved in $90.56 \%$ reports in the first group whereas $94.5 \%$ reports in the second group.

CONCLUSIONS: We observed decreased TAT and better target TAT values by using Barricor ${ }^{\mathrm{TM}}$ tubes and prompt centrifugation, for the analysis of high sensitivity troponin I tests.

Key Words: plasma, test tube, turn around time, pre-analytical phase, quality improvement

\section{ARE ALL BIOCHEMICAL PARAMETERS AFFECTED BY EDTA CONTAMINATION?}

\author{
$\underline{\text { A. Maresch }}{ }^{1}$, C. Pereira ${ }^{1}$, C. Queiroz ${ }^{1}$, T. Rodrigues ${ }^{1}$, F. Escada ${ }^{1}$, R. Monteiro ${ }^{2}$, L. Araújo ${ }^{1}$, J. Pego ${ }^{1}$, F. Rodrigues ${ }^{1}$ \\ ${ }^{1}$ Centro Hospitalar e Universitário de Coimbra, Coimbra, Portugal \\ ${ }^{2}$ Centro Hospitalar do Baixo Vouga, Aveiro, Portugal
}

BACKGROUND-AIM: Studies state that most laboratory errors are due to pre-analytical factors. Among others, one of these factors is the contamination of lithium heparin tubes with K3-EDTA in the blood collection process, typically recognized for brisk elevation of potassium and calcium chelation resulting in very low calcium levels. The aim of this study was to evaluate the influence of EDTA contamination in several clinical chemistry tests.

METHODS: Twenty-two patient samples were selected, based on EDTA contamination followed by prompt re-evaluation (maximum: 7 hours) of the biochemical parameters: sodium (Na2+), chloride (Cl-), creatinine, aspartate transaminase (AST), alanine transaminase (ALT), alkaline phosphatase (AP), gamma-glutamyltransferase (GGT) and C-reactive protein (CRP). All data was analysed with SPSS 25 (IBM Corp.).

RESULTS: No statistical differences were found between the successive measurements of creatinine, AST, ALT, GGT and CRP ( $p<0.05$ ). Strong linear correlation was achieved in creatinine, Cl-, AST, ALT, GGT and CRP $(\mathrm{R} \geq 0.80)$. Na2+ sustained a moderate correlation amongst the consecutive samples, with significant fluctuations in some patients. AP varied importantly, with markedly lower values measured in the EDTA tube (increasing between $59-86 \%$ in the re-evaluation sample, $95 \%$ confidence interval).

CONCLUSIONS: Based on the results, in cases of EDTA contamination, creatinine, Cl-, AST, ALT, GGT and CRP results can predict the patient's status as well as the lithium heparin without contamination. The correlation in Na2+ results is moderate. This can be due to the EDTA contamination, but one must consider the high variability of these with fluid therapy that could have been instituted between samples. AP varied significantly, which can be explained by AP, a zinc-containing metalloenzyme, suffering irreversible inactivation after exposure to EDTA, probably due to the removal of zinc atoms.

Key Words: EDTA, contamination, correlation 


\title{
SELF-SAMPLING ON FAECAL MATERIALS. PRODUCTION OF DEVICES AND REPORT OF PERFORMANCE. PROPOSAL OF UNIT TO REPORT DATA AND HARMONIZE DEVICES
}

\author{
$\underline{\text { S. Rapi }}{ }^{1}$, A. Bonari ${ }^{3}$, F. Cellai ${ }^{2}$, 1. Chelazzi ${ }^{3}$, S. Ciattini ${ }^{3}$, T. Rubeca ${ }^{2}$ \\ ${ }^{1} \mathrm{AOU}$ Careggi, Florence, Italy \\ ${ }^{2} I S P R O$, Institute for Cancer Reseanch and Network, Florence, Italy \\ ${ }^{3}$ University of Florence, Florence, Italy.
}

BACKGROUND-AIM: Quantitative methods on faeces are based on self-sampling devices produced by manufacturers without technical and metrical indication; in this study we present the steps to produce and evaluate performances of sampling dipsticks and propose the use of correct units to report data.

METHODS: Fifty prototypes of 3 types of dipsticks (D1; D2; D3) with dimples repeated 1, 2 or 3 folds and target volume 5;10;15 mm3 were produced on 3D printer. Volumes of sampling regions was measured on X-ray microtomography. Mean and imprecision of collected mass was measured by gravimetry in 2 different experiments on faecal materials from 2 health volunteers; 8 measurements was performed for D1; D2; D3 to obtain the mean mass of recovered materials. Mean values from the two runs was used to obtain the inter-run imprecision for devices. Ratio between mean mass of collected faeces and X-ray volumes was calculated. Percentage between collected and targeted amount was used as indicator of performance.

RESULTS: Sampling volumes of dipsticks on X-ray results D1 $=5 \pm 02$; D2 $=8.7 \pm 0.2$; D $3=15 \pm 0.2 \mathrm{~mm}$.Intra-assay imprecision (mean $\pm \mathrm{sd}$ ) of dipsticks results D1 $=4.46 \pm 0.55 \mathrm{mg}$; $\mathrm{D} 2=7.7 \pm 0.46 \mathrm{mg}$; $3=12.13 \pm 0.4 \mathrm{mg}$ for run 1 and $4.47 \pm 0.38 \mathrm{mg} ; 8.08 \pm 1.15 \mathrm{mg} ; 12.50 \pm 0.2 \mathrm{mg}$ for run 2 , Inter-assay imprecision results D1 $=4.46 \pm 0.45$; D2 $=7.87 \pm 0.76$; D $3=12.28 \pm 0.36$. Ratio between collected materials and measured sampling volumes results $0.90 \mathrm{mg} / \mathrm{mm} 3$ for D1; $0.93 \mathrm{mg} / \mathrm{mm} 3$ for D2 and $0.82 \mathrm{mg} / \mathrm{mm} 3 \mathrm{D} 3$ whereas \% between collected and targeted results $90 \%$; $81 \%$ and 83\% for D1; D2; D3.

CONCLUSIONS: Design and production of devices with known performances is possible and should be done to harmonize pre analytical on self-sampling based fecal methods. Ratio between collected amount of faeces and sampling volumes was reported close to 1 also on commercial sampling dipsticks, The volume of the sampling regions ( $\mathrm{mm} 3)$ is the correct unit to report data on self-sampling based methods.

Key Words: faecal test sampling, reporting units, sampling devices

\section{UNDERSTANDING NURSING PREANALYTICAL NEEDS TO FIX BLOOD VENOUS SAMPLING PROBLEMS}

\author{
M. Gorbatsevich ${ }^{1}$, G. Lima-Oliveira ${ }^{1}$ \\ ${ }^{1}$ Latin American Working Group for Preanalytical Phase (WG-PRE-LATAM) of the Latin America Confederation of Clinical Biochemistry (COLA- \\ BIOCLI), Montevideo, Uruguay
}

BACKGROUND-AIM: The aim of this work was to evaluate the nurse knowledge of essential preanalytical issues for blood venous sampling in Belarus.

METHODS: A self-report survey regarding blood venous sampling were delivered to all nurses that attended the IV International Summit of medical nurses in Minsk together with Ministry of Health of Belarus and Russia. Briefly, the self-reported survey had 7 questions where multiple answers were allowed: Q1. What devices are used for venous blood collection in your hospital?; Q2. What is the most important characteristic of a vacuum system from your point of view?; Q3. How often vacuum tubes unfilled till the mark?; Q4. What are your actions, if the evacuated tube for coagulation (blue stopper) does not collect the sample up to the mark?; Q5. Do you discard the evacuated tube if it is unfilled till the mark after sampling?; Q6. What brands of evacuated tubes you are using?; Q7. What difficulties have you experienced when you mixed components (needle, holder, butterfly, tubes etc) of evacuated tubes systems from different manufacturers?

RESULTS: 363 nurses answered the survey. The frequency of answers for each question (Q1 to Q7) that could jeopardize patient safety or can be an opportunity to improve the nurse practices are listed: Q1. 19\% use syringe then fill blood into evacuated tubes; Q2. 62\% safety, 32\% sterility, 7\% low price; Q3. 10\% constantly, i.e. more than 3 times per 100 tubes; Q4. 10\% mix blood from two sodium citrated tubes to obtain the nominal volume into one tube; Q5. 21\% never; Q6. 26\% SANLI, 21\% Greiner Bio-One; Q7. 18\% incompatibility of components.

CONCLUSIONS: Nurses evaluated related to perform some "bad" practices during blood venous sampling for laboratory testing. However, we believe that the root cause is the lack of knowledge regarding preanalytical variability sources. In the future, the Joint EFLM-COLABIOCLI Recommendation for venous blood sampling could help to improve the quality of nurse procedures and guarantee patient safety in our countries.

Key Words: blood specimen collection, medical errors, patient safety, phlebotomy, specimen handling 


\title{
ASSESSMENT OF PRE-ANALYTICAL ERRORS IN COLLECTION OF BLOOD SAMPLES FOR MOLECULAR TESTING
}

\author{
W. Tamimi ${ }^{1}$, R. Sarr ${ }^{1}$, M. Alkurdi ${ }^{2}$ \\ ${ }^{1}$ King Abdulaziz Medical City, Riyadh \\ ${ }^{2}$ Pranaltical scinces $B D$
}

BACKGROUND-AIM: There is a lack of guidelines for collection, handling, stabilization and storage of patient's samples in molecular assays. In absence of these guidelines, collection of blood samples are subjected to many errors in particular for molecular assays. In an attempt to improve our collection guidelines and reduce preanalytical errors for molecular testing, we have assessed the collection procedures in many hospital locations that performed by heath care providers.

METHODS: A total of 3258 blood samples were received for molecular testing between years 2012 and 2013. Most samples were collected and transported to the laboratory by nurses. Samples were evaluated for any problems. Samples problems were classified as mislabeled, unlabeled, contaminated, Incorrect tube, wrong patient extracted, sample without request, sample broken in the centrifuge, transport problems, specimen reception problems, accession problems, quantity not sufficient (QNS), clotted, and hemolysed specimen.

RESULTS: Many problems were raised during and after the collection of blood for molecular testing. There were 142 (4.4\%) specimen errors including 4 with transport, 68 with accession, 23 with reception problems, 9 with hemolysed samples, 13 with clotted specimen, 10 with QNS and 15 with sub-optimal DNA/ RNA.

CONCLUSIONS: Establishing guidelines for the preanalytical phase of collecting blood samples used for molecular testing is mandatory as there are lack of standardization and more new lab and testing are required. Survey and EQC program should be considered for pre-analytical molecular assays. Stability of blood samples for molecular lab must be maintained in order to produce a good quality results.

Key Words: preanalytical phase

\section{SAMPLE MATRIX INTERFERENCES - CHOOSING THE PROPER PLASMA SAMPLE TYPE FOR TESTING MARKERS FOR INFECTIOUS DISEASES}

\author{
N. Chokrevska Zografska ${ }^{1}$, V. Dirjanska-Filipche ${ }^{1}$, D. Stevanoska ${ }^{1}$, S. Rajovski ${ }^{1}$, K. Stojanovska ${ }^{1}$ \\ ${ }^{1} \mathrm{CH}$ Acibadem Sistina, Diagnostic laboratories, Biochemistry department, Skopje, Macedonia
}

BACKGROUND-AIM: According to the latest propositions heparin plasma was used as analytical matrix instead of serum. But, we faced difficulties in the analytical phase for the markers for infectious diseases HBsAg, anti HCV and HIV Combi test. The results were discrepant from previous result for the same patient, or were false positive with a small cut-off index value, up to 5.0, for all 3 tests. The aim is to present the importance of the choice of the most suitable plasma sample type for testing markers for infectious diseases.

METHODS: The analysis were performed on immunology analyzer Siemens ADVIA Centaur XPT, CLIA method, and QC results were within defined ranges of uncertainty of measurement. Blood was routinely drawn in sample tubes BD heparin plasma with gel separator, Cat. No. 366567, Lot No. 8116771 and BD EDTA plasma Cat. No. 368841, Lot No. 8050709.

RESULTS: Routine and dialysis patient samples analyzed in heparin plasma with false positives with a small cut-off index value, up to 5.0, were tested in repeats in EDTA plasma. In total of 7837 results for HBsAg, 80 tests, $1.02 \%$ were repeated, out of 7823 results for anti HCV, 24 tests, $0.306 \%$ were repeated, and out of 7779 results for HIV Combi, 26 tests, $0.334 \%$ were repeated and all were negative. The comparison study showed high statistical difference, in this case $100 \%$.

CONCLUSIONS: The quality of plasma sample type has big influence on precision and accuracy of the measured parameters for all patients and was the reason for changing to EDTA plasma for testing these analyses. Choosing the proper sample matrix for analysis, as well as continuous following of the quality indicators in the pre-analytical phase, is a must in the implementation of the recommendations for the preanalytical phase and thus, upgrading of the whole quality control processes in the laboratory.

Key Words: pre-analytical phase, sample quality, sample matrix, heparin plasma, EDTA plasma, immunology tests

\section{IS PLASMA ALWAYS A SUITABLE ALTERNATIVE TO SERUM IN A LARGE TEST PANEL}

\author{
$\underline{\text { S. Muhtaroglu }}^{1}$, D. Barlak Keti ${ }^{1}$ \\ ${ }^{1}$ Department of Medical Biochemistry, Erciyes University Faculty of Medicine, Kayseri, Turkey
}

BACKGROUND-AIM: Serum is the most widely used sample in biochemical analysis, but plasma has some advantages when compared to serum such as reduction in turnaround time, no fibrin and gel-based interference and more accurate reflection of the in vivo situation. The aim of this study was to evaluate whether there is a difference between serum and plasma for 31 analytes. 
METHODS: Total of 50 healthy subjects were included in the study. Blood samples were collected in tubes with gel and containing lithium heparin (Barricor). Samples in tubes with gel were allowed to clot at room temperature. Serum and plasma were obtained by centrifugation at $2000 \mathrm{~g}$ for 10 minutes. Hemolysis index was lower than 30 in serum and plasma samples. 25 biochemical measurements and 6 immunoassay parameters were performed within two hours on Cobas c702 and on Cobas e602, respectively (Roche Diagnostics GmbH, Mannheim, Germany). RESULTS: Lactate dehydrogenase (LDH), creatine kinase (CK-MB) activities, folate, and total protein levels were significantly higher $(\mathrm{p}<0.001)$ although potassium and phosphate were significantly lower $(\mathrm{p}<0.001)$ in plasma when compared to serum. LDH, CK-MB, folate, total protein, potassium and phosphate had $12.3 \%, 16.6 \%, 10.6 \%, 3.3 \%,-7.8 \%$ and $-10.3 \%$, and bias in plasma, respectively relative to the serum.

CONCLUSIONS: The use of serum reference ranges is not suitable for plasma LDH, CK-MB, potassium, phosphate, folate and total protein measurements. Plasma is a better quality sample because it is independent of fibrin and gel interference. However, the stability of analytes dependent on time and temperature in plasma should also be taken into consideration. Each laboratory may prefer serum or plasma according to their own conditions.

Key Words: serum, plasma, biochemical analysis

\title{
CAN SERUM AND EDTA-PLASMA SAMPLES BE USED INTERCHANGEABLY FOR HIGH SENSITIVE TROPONIN I MEASUREMENT?
}

\author{
E. Jurčić-Karlović ${ }^{1}$, S. Fanda ${ }^{1}$, M. Tomas ${ }^{1}$, I. Kuktić ${ }^{1}, \underline{\text { A. Dojder }}^{1}$, V. Radišić Biljak ${ }^{1}$, A. Šimundić ${ }^{1}$ \\ ${ }^{1}$ Department of Medical Laboratory Diagnostics, University Hospital “Sveti Duh”, Zagreb, Croatia
}

BACKGROUND-AIM: Manufacturer (Beckman Coulter, USA) recommends serum, EDTA and Li-heparin plasma for high sensitive troponin I (hsTnI) measurement, with a conversion factor for serum values of 0.90 for 99 th percentile upper reference limit (URL) of a healthy population when EDTA plasma samples are used. The aim of the study is to verify the manufacturer's claims for measurement of hsTnI in EDTA plasma samples.

METHODS: High sensitive TnI was measured in both serum and EDTA-plasma samples of 31 patients with suspected AMI admitted to our Emergency Department. Measurements were done by chemiluminescent method on DXI 600 (Beckman Coulter, USA) analyzer. The 99th percentile URL defined by manufacturer is 17.5 (12.6-20.7) $\mathrm{ng} / \mathrm{L}$. Method CV(\%) at a lower reference limit of 99th percentile (12.2 ng/L) was estimated in accordance to CLSI EP15-A3 guideline. According to IFCC Committee on Clinical Applications of Cardiac Bio-Markers our acceptance criterion for method precision was $\leq 10 \%$ for 99th percentile URL of a healthy population. The hsTnI concentrations were compared by nonparametric Wilcoxon test. P-value $<0.05$ was considered significant. Also, the comparability of results was assessed by linear regression. Statistical analysis was done in MedCalc Statistical Software v16.2.0 (MedCalc Software bvba, Ostend, Belgium).

RESULTS: Method precision at $12.2 \mathrm{ng} / \mathrm{L}$ was $8.55 \%$. There was a statistically significant difference in median values (in ng/L) of serum [15.20 (9.53-32.49)] and EDTA-plasma samples [12.30 (7.63-26.89)], $\mathrm{P}<0.001$. Median bias was $2.6 \mathrm{ng} / \mathrm{L}$ (95\% Cl: 1.50-4.68). The regression coefficients were: 0.75 (95\% CI:-1.80-3.29, P=0.554) for intercept, and 0.89 (95\% CI: 0.8941-0.8952, P < 0.0001) for slope.

CONCLUSIONS: Our study confirmed the manufacturer's conversion factor of 0.90 for measurement of hsTnI in EDTA plasma samples. The serum and EDTA plasma samples for hsTnI measurement should not be used interchangeably.

Key Words: hsTnI, sample, preanalytical phase

\section{Hemolysis, icterus, lipemia}

\section{EFFECTS OF HAEMOLYSIS, ICTERUS AND LIPAEMIA ON THE ABBOTT ARCHITECT TOTAL BILE ACIDS ASSAY}

\author{
$\underline{\text { C.K.M. Ho }}^{1}$, K.Z.J. He ${ }^{2}$, S.P. Lim $^{2}$, J.W.S. Setoh ${ }^{1}$ \\ ${ }^{1} \mathrm{KK}$ Women's and Children's Hospital, Singapore, Singapore \\ ${ }^{2}$ Ngee Ann Polytechnic, Singapore, Singapore
}

BACKGROUND-AIM: Serum total bile acids levels are useful for the assessment of hepatobiliary diseases including obstetric cholestasis. This study aimed to investigate potential interference of the Abbott Architect total bile acids assay by haemolysis, icterus and lipaemia (HIL).

METHODS: Serum total bile acids levels in residual samples were measured using an automated, enzymatic-colorimetric assay (Architect c8000, Abbott Laboratories). Pooled serum samples with either 10 or 40 ( mol/L were spiked with haemolysate, unconjugated bilirubin or Lipofundin to achieve up to $5250 \mathrm{mg} / \mathrm{L}$ haemoglobin, 855 (mol/L bilirubin or $11 \mathrm{mmol} / \mathrm{L}$ triglycerides, respectively. Semi-quantitative indices for HIL were measured on the same instrument according to the manufacturer's protocol. Deviations of total bile acids levels in spiked samples more than $10 \%$ from their target values were considered clinically significant. 
RESULTS: In serum samples with target bile acids levels of 10 ( mol/L, mild haemolysis (haemoglobin $\geq 500 \mathrm{mg} / \mathrm{L} ; \mathrm{H}$ index +1 or higher) increased, whereas mild lipaemia (triglycerides $\geq 0.68 \mathrm{mmol} / \mathrm{L}$; L index +1 or higher) decreased the measured bile acids levels. In comparison, in samples with target bile acids levels of $40 \mathrm{fmol} / \mathrm{L}$, clinically significant assay interference was observed in the presence of severe haemolysis (haemoglobin $5250 \mathrm{mg} / \mathrm{L}$; H index $4+$ ) or moderate lipaemia (triglycerides $\geq 1.36 \mathrm{mmol} / \mathrm{L}$; L index +2 or higher). Icterus only slightly affected the measured bile acids levels (target: $10(\mathrm{~mol} / \mathrm{L})$ at very high concentrations of bilirubin (855 $\int \mathrm{mol} / \mathrm{L}$; I index 4+).

CONCLUSIONS: Clinically significant interference of the total bile acids assay was observed in the presence of even mild haemolysis or mild lipaemia. The effect of bilirubin on the total bile acids assay was minimal. Appropriate pre-analytical procedures and preparation of patients are of paramount importance for the accurate measurement of total bile acids using the Abbott Architect assay.

Key Words: hemolysis, icterus, lipaemia, bile acids

\title{
HANDLING IRON REQUESTS ON HAEMOLYSED SAMPLES
}

\author{
$\underline{\text { R. Hawkins }}^{1}$ \\ ${ }^{1}$ Laboratory Medicine, Tan Tock Seng Hospital, Singapore
}

BACKGROUND-AIM: As sample haemolysis invalidates iron measurement due to positive interference from free Hb, it is good practice to reject such samples. We follow this approach but leave it up to the requesting clinician to decide on the need for retesting. This study describes the pattern of iron requests in a 1200 bed general hospital, the prevalence of haemolysis, the pattern of subsequent retesting and the effect of simultaneous ferritin requesting. Most requests are for evaluation of anaemia rather than iron overload in our Asian population.

METHODS: Details of iron and ferritin inpatient requests for 3 years were extracted from the laboratory information system for analysis in Excel and Access. Fe and ferritin are measured on 3 Beckman Coulter DxC-800 (Fe) and 2 DxI-800 (ferritin) analysers. All samples undergo haemolysis index (HI) measurement and the Fe result is suppressed if $\mathrm{HI}>=1$.

RESULTS: There were 37631 Fe requests, 94\% with simultaneous ferritin requesting. The rate of suppressed Fe results due to haemolysis and subsequent Fe re-requesting within 7 days was $16.3 \%$ and $42 \%$. The repeat rates were lower with no simultaneous ferritin requested (28.5\%) vs Fe with ferritin together (43.1\%). Repeat testing rates remained high despite clear initial diagnostic ferritin results (e.g. ferritin < 12 ug/L, repeat rate $19 \%$; ferritin $>200 \mathrm{ug} / \mathrm{L}, 32 \%$ ).

CONCLUSIONS: Suppressed Fe results due to haemolysis occur in 16\% of inpatient samples with 40\% recollected. Reporting simultaneous ferritin did not reduce Fe re-requesting rates. Repeat testing occurred in $20 \%$ of cases despite low ferritin results consistent with iron deficiency anaemia. Review of re-requesting procedures and clinician education is needed to improve test utilisation.

Key Words: haemolysis, iron, phlebotomy

\section{EVALUATION OF THE PREANALYTICAL, AUTOMATED IDENTIFICATION OF LIPEMIC SPECIMENS FOR HEMOSTASIS TESTING}

\author{
$\underline{\text { A. Kotanidou }}^{2}$, M. Theocharidou ${ }^{2}$, T. Midioura ${ }^{1}$, P. Aslanoglou ${ }^{2}$, A. Agorasti ${ }^{2}$ \\ ${ }^{1}$ Biochemistry Laboratory, General Hospital of Xanthi, Xanthi, Greece \\ ${ }^{2}$ Haematology Laboratory, General Hospital of Xanthi, Xanthi, Greece
}

BACKGROUND-AIM: Lipemia interferes in hemostasis testing. According to the reference guide (compliance sheet) of the Thromborel ${ }^{\circ} \mathrm{S}$ reagent (SIEMENS) in use on the Sysmex ${ }^{\circledR}$ CS-2100i Hemostasis system, triglycerides concentration below $203 \mathrm{mg} / \mathrm{dL}$ does not interfere in the measurement of Prophtombin Time INR. Sysmex ${ }^{\circledR}$ CS-2100i Hemostasis system detects hemolysis, icterus and lipemia using a preanalytic scan (multi-wavelength Preanalytical Sample Integrity) and the problematic specimen is flagged as "Hem”, "Ict" or "Lip". The aim of this study is the evaluation of the automated identification of lipemic specimens by CS-2100i coagulation analyzer.

METHODS: We recorded retrospectively the samples with INR measurement and triglycerides concentration above the cut-off value (> 203 mg/dL) analyzed on Sysmex® CS-2100i during October 2019. Then, we searched on the analyzer if those samples were flagged as "Lip” (means lipemic specimen).

RESULTS: Twenty-eight samples presented triglycerides concentration that interferes in the measurement of INR. Only three samples with triglycerides concentration 1160, 1090 and $1060 \mathrm{mg} / \mathrm{dL}$ were flagged as unsuitable "Lip" (10,7\%). The remaining samples with triglycerides concentration between $300-858 \mathrm{mg} / \mathrm{dL}$ were not marked as “Lip” (89,3\%).

CONCLUSIONS: Sysmex ${ }^{\circledR}$ CS-2100i identified only the $10,7 \%$ of the lipemic specimens using the automated preanalytic scan. The identification and the management of unsuitable samples due to lipemia seems to be a complex issue and Laboratory should establish a check prior to analysis.

Key Words: hemostasis testing, lipemia, specimen condition 


\title{
THE HILS ARE ALIVE WITH THE SOUND OF INNOVATION
}

\author{
$\underline{\text { R. Marrington }}^{1}$, J. French ${ }^{1}$, F. Mackenzie ${ }^{1}$ \\ ${ }^{1}$ Birmingham Quality [UK NEQAS], University Hospitals Birmingham NHS Foundation Trust, Birmingham
}

BACKGROUND-AIM: The presence of Haemoglobin (Haemolysis), Bilirubin (Icterus) and Lipids (Lipaemia) in serum can affect the measurement and reporting of many Clinical Chemistry laboratory test results. This is not only in the validity of the test results themselves, but also whether a result is, or should be reported. Quality Assurance of Serum Indices is not as rigorous as for other Clinical Chemistry assays.

METHODS: Birmingham Quality has established UK NEQAS for Serum Indices (HIL) which is an EQA scheme that not only looks at Haemolysis, Icterus and Lipaemia as individual analytes but also looks at the impact these serum indices have on a particular specific analyte, which changes from month to month, called 'Analyte X'. Laboratories return whether they would report the measured analyte based on the serum indices.

RESULTS: For a number of analytes, the results returned so far have shown that there are significant differences in practice for the interpretation of serum indices both within-, and between- manufacturers. For example, three specimens were distributed with varying degrees of haemolysis for the analysis of Total Protein. The grossly haemolysed specimen would have had its Total Protein result reported by 57\% of participants. This was not wholly due to between manufacturer differences. Haemolysis did adversely affect the Total Protein result for one manufacturer but there were many participants using this method that did report the result when most other Laboratories/Systems would not have. Since Total Protein may be used in the calculation of Globulins or calculations of amount of monoclonal proteins which may trigger subsequent additional unnecessary testing.

CONCLUSIONS: The UK NEQAS for Serum Indices (HIL) has shown that there are significant variations in practice between participants for the use of serum indices which consequently affects patient care both in terms of repeat testing and validity of test results.

Key Words: serum indices, HIL, EQA

\section{LIPEMIA INTERFERENCE ON NEPHELOMETRY ASSAYS}

\author{
$\underline{\text { M. Njegovan }}^{1}$, A. Bokulić ${ }^{2}$, L. Đerek ${ }^{1}$, A. Tešija Kuna ${ }^{1}$, I. Vukasović ${ }^{1}$, V. Vidranski ${ }^{2}$ \\ ${ }^{1}$ Department of Clinical Chemistry, Sestre milosrdnice University Hospital Center, Zagreb, Croatia \\ ${ }^{2}$ Department of Oncology and Nuclear Medicine, Sestre milosrdnice University Hospital Center, Zagreb, Croatia
}

BACKGROUND-AIM: The aim of study was to verify lipemia interference criteria declared by manufacturer for ceruloplasmin, albumin, immunoglobulin G (IgG), immunoglobulin free light chains (FLC) kappa and lambda assays and to evaluate usefulness of serum lipemic index (L index). Lipemia interference study was performed on the BN II nephelometer System (Siemens Healthcare Diagnostics Inc., USA).

METHODS: Sample preparation of lipemia interference was simulated using SMOFLipid solution 20\% (Fresenius Kabi AB, Uppsala, Sweden) and serum pool. L index and triglycerides concentration were measured on Abbott Architect C8000 (Abbott, Abbott Park, Illinois, USA) . Deviation of expected results higer than the establised measurement uncertainty (MU) for assays was considered as clinicaly significant deviation. For ceruloplasmin MU is 8\%, albumin 13\%, IgG 11\%, FLC kappa 9\% and FLC lambda 12\%.

RESULTS: Measured triglycerides (with corresponding measured L-index) and declared triglycerides interferences were as follows: for ceruloplasmin $3.5 \mathrm{mmol} / \mathrm{L}$ (1.1) and $2.7 \mathrm{mmol} / \mathrm{L}$, albumin $24.1 \mathrm{mmol} / \mathrm{L}$ (9.4) and $22.6 \mathrm{mmol} / \mathrm{L}$, IgG $24.1 \mathrm{mmol} / \mathrm{L}$ (9.4) and $21.5 \mathrm{mmol} / \mathrm{L}, \mathrm{FLC} \mathrm{kappa}$ $24.1 \mathrm{mmol} / \mathrm{L}$ (9.4) and $5.7 \mathrm{mmol} / \mathrm{L}$ and FLC lambda $7.8 \mathrm{mmol} / \mathrm{L}$ (2.3) and $5.7 \mathrm{mmol} / \mathrm{L}$.

CONCLUSIONS: For all assays triglycerides concentrations that interfere with results were found higher than the declared one. Lipemia interference is manifested as a positive bias for ceruloplasmin and IgG and negative bias for albumin and FLC kappa and lambda. Lipemia can cause erroneus results in nephelometry assays and measuring $\mathrm{L}$ index with an automated assay is very usefull for defining pre-analitic acceptance criteria for chosen assays.

Key Words: L-index, interference, nephelometry

\section{HANDLING HEMOLYZED PEDIATRIC SAMPLES: OUR EXPERIENCE}

$\underline{\text { E. Stenner }}^{1}$, M. Ruscio ${ }^{1}$

${ }^{1}$ SC Laboratorio Unico di ASUITs, Burlo, Gorizia e Monfalcone, Dipartimento di Medicina dei Servizi, Azienda Sanitaria Universitaria di Trieste, Italy

BACKGROUND-AIM: Pediatric phlebotomy is an every day challenge: it is invasive and can cause possible long-term negative effects (i.e reduced pain tolerance). Previous papers described that pediatric collections are often severalfold more likely to be hemolyzed both for inpatients and outpatients. Test results suppression in hemolyzed samples is associated with many clinical, economic, and organizational consequences, above all for pediatric populations. The aim of our study was to optimize our pediatric rejection sample rate.

METHODS: Since January 2017, an automated measurement of hemolysis (H-index) was used for all pediatric serum samples (semi-quantitative photometric test - Beckman Coulter - AU analyzers). Manufactureres' H-index cut-offs for flagging, alarming or suppressing test results 
were applied. Algorithms to manage hemolysis-sensitive test results (HSTR) were applaied and specific comments to add to the laboratory reports were defined. Specifically, all HSTR with a bias inferior to reference change value were reported with an associated specific comment (i.e hemolozied sample +1 . Value possible increased). Before implementing algorithms and interpretative comments, a meeting with the emergency department was performed.

RESULTS: Our data (mean number of patients below 10 years: 307 [276-405]) evidenced that there was a substantial percentage of hemolized results $(4.3 \%$ [3.1 - 7.9]). About $40 \%$ of these results were reported in any case but with specific interpretative comments.

CONCLUSIONS: Our experience let us to consider safe the implementation of an automated algorithm based on decision-making rules to manage HSTR. It may reduce the rejection sample rate optimizing, as a consequence, the turn-around-time. Moreover, the use of these algorithm standardize the technicians behavior reducing the risk of errors in this part of the intra-laboratory pre-analytical phase.

Key Words: haemolysis, pediatric, report

\title{
EVALUATION OF A SERUM INDICES QUALITY CONTROL FOR PRE-ANALYTICAL MONITORING
}

\author{
M. Gonzales ${ }^{1}$, H. Onishi ${ }^{1}$, L. Wong ${ }^{1}$ \\ ${ }^{1}$ Bio-Rad Laboratories, Inc., Irvine, USA
}

BACKGROUND-AIM: Pre-analytical monitoring of instrumentation performance is an important step in the assessment of patient specimens. Specimens compromised by improper handling, and other factors can interfere with an assay's performance. Three of the most common specimen interferences that contribute to pre-analytical variation are Hemolysis (H), Icterus (I), and Lipemia (L). For many years, detection of potential specimen interferences was reliant on a visual assessment by laboratory personnel. Automated detection of serum indices is more accurate than visual assessment and the instruments used for detection require periodic calibration. However, until recently, commutable, commercially produced human sera derived quality control materials were not available for routine instrument monitoring. This study evaluates such a product on selected popular instrument platforms.

METHODS: Bio-Rad Liquichek Serum Indices (H, I, L) were tested in replicate on the Abbott ARCHITECT, Beckman AU, Ortho VITROS, Roche COBAS and Siemens VISTA instruments following manufacturer's instruction for patient specimen pre-analytical assessment.

RESULTS: Liquichek Serum Indices produced relevant levels of interference similar to that of a patient sample. Results as follows: Ortho VITROS, Abbott ARCHITECT, Roche COBAS: 100-300 Hemolysis Index, 10-30 Icterus Index, 150-550 Lipemia Index; and Beckman AU, Siemens VISTA: 2-5+ Hemolysis Index, 3-6+ Icterus Index, 3-5+ Lipemia Index.

CONCLUSIONS: Results demonstrate the functionality, commutability and clinical utility of the third-party Liquichek Serum Indices product. Liquichek Serum Indices monitors an instruments' ability to detect potential interferences through the HIL pre-analytical test function, thereby increasing the reliability of test results, and ultimately improving patient care.

Key Words: QC, commutability, interference, hemolysis, lipemia

\section{HEMOLYSIS INDEX - PERFORMANCE ESTIMATION AT TWO CLINICAL CHEMISTRY PLATFORMS}

J. Štanfel ${ }^{1}$, M. Milić ${ }^{1}$, V. Šerić ${ }^{1}$

${ }^{1}$ Department of Clinical Laboratory Diagnostics, Osijek University Hospital, Osijek, Croatia

BACKGROUND-AIM: Automated semi quantitative estimation of hemolysis - hemolysis index (HI) is available on almost all clinical chemistry platforms. The performance differs in specimen dilution, wave length, different measuring range and report. We compared assessment of HI at two platforms to verify if the results lead to same interpretation.

METHODS: HI was performed in 54 patient samples with visually established hemolysis on two platforms, AU680 (Beckman Coulter) and Dimension ExL (Siemens). HI levels are set by the manufacturers as follow: AU680 - N (<0.5 g/L), 1 (0.5-0.99 g/L), 2 (1.0-1.99 g/L), 3 (2.0-2.99 g/L), 4 (3,0-4.99 g/L), 5 (>5.0 g/L); Dimension ExL -1 (<0.25 g/L), 2 (0.25-0.5 g/L), 3 (0.5-2.0 g/L), 4 (2.0-3.0 g7/L), 5 (3.0-5.0 g/L), 6 (5.0-10.0 g/L) RESULTS: HI was determined in 54 samples at both platforms, but for 10 samples (18.52\%) at AU680 abnormal result (ABN) is obtained 7 samples ( $14.28 \%$ ) with concentration lower than $0.5 \mathrm{~g} / \mathrm{L}$ and 3 samples ( $5.55 \%$ ) higher than $5.0 \mathrm{~g} / \mathrm{L}$. Remaining 44 samples (81.48 \%) belong to the HI level with same concentration range. Out of 54 samples, 11 (20.38\%) belong to the level that overlap in the whole concentration range and 30 ( $55.55 \%)$ to the level that overlap partially. Out of 54, 3 samples (5.55\%) belong to different HI level ranges that overlap in one concentration point set on the cut off value.

CONCLUSIONS: Automatic inspection of HI has made hemolysis detection more reliable. Lack of harmonization may lead to different result interpretation, due to method linearity and especially sensitivity. Consequently, samples with very low degree of hemolysis could be rejected. Cut offs and concentration ranges are not equally distributed through the whole measurement range and the reports are not equal. Establishing HI threshold for sample rejection could lead to the same sample being accepted on the one platform and rejected on the other. Need for harmonization of all measurement segments and reporting $\mathrm{HI}$ is necessary.

Key Words: hemolysis index, harmonization, automatisation 


\title{
HAEMOLYSIS IN PREMATURES AND NEONATES: A PREANALYTICAL CHALLENGE
}

\author{
L. Tóth ${ }^{1}$, T. Kovács ${ }^{2}$, E. Kalina ${ }^{1}$, E. Szakács Szilágyi ${ }^{1}$, J. Kappelmayer ${ }^{1}$ \\ ${ }^{1}$ Department of Laboratory Medicine, Faculty of Medicine, University of Debrecen, Debrecen, Hungary \\ ${ }^{2}$ Department of Pediatrics, Faculty of Medicine, University of Debrecen, Debrecen, Hungary
}

BACKGROUND-AIM: Haemolysis is the most frequent cause of test or specimen rejection in clinical laboratories. It is mainly in vitro haemolysis which can be reduced by education and trainings. In case of prematures and neonates, in vivo haemolysis may also exist besides in vitro haemolysis due to the complicated blood sampling. This patient group may have the most severe haemolysis which in some cases can interfere also with CRP measurement which is very important to monitor during perinatal infections.

METHODS: In all serum samples arrived for routine and stat clinical chemical and immunochemical assays in 2017, haemolysis index was measured spectrophotometrically, and the ratio of haemolysed samples and the test rejection rates were analysed. Haemolysed sample was defined as the free haemoglobin concentration of the sample was higher than $0,5 \mathrm{~g} / \mathrm{L}$. Test rejection rate was calculated as the number of tests rejected by haemolysis compared to the total test number. Data of prematures-neonates and other patients were analysed separately. RESULTS: Haemolysis index was measured in case of 285420 serum samples in 2017. 98.5\% of the samples was collected from general patient population and $1.5 \%$ from prematures and neonates. The ratio of haemolysed samples was 3.51\% in general patient population and 50.93\% in prematures-neonates, while the test rejection rates by haemolysis were $0.61 \%$ and $3.56 \%$, respectively. The mean haemolysis index of the samples of prematures and neonates was 70. In 17 cases the CRP test was rejected by severe haemolysis. Repeating the blood sampling, all of these CRP tests became measurable.

CONCLUSIONS: In case of prematures and neonates, the haemolysis is a more important preanalytical problem than in general patient population, and blood collection trainings have a greater impact to reduce severe in vitro haemolysis.

Key Words: haemolysis, test rejection, prematures, neonates

\section{PROLONGED PROTHROMBIN TIME DUE TO POSTPRANDIAL HYPERTRIGLYCERIDEMIA: A CASE REPORT}

\author{
E. Refatllari ${ }^{1}$, H. Lame ${ }^{1}$, A. Coraj ${ }^{1}$, N. Heta ${ }^{1}$, A. Bulo ${ }^{1}$ \\ ${ }^{1}$ Laboratory Department, University Hospital Center "Mother Teresa”, Tirana, Albania
}

BACKGROUND-AIM: The aim of this report is to present a case of falsely prolonged prothrombin time (PT) in a lipemic sample which can directly affect the patient's treatment. The most common preanalytical cause of lipemia is inadequate time of blood sampling. Lipemia interference in optical clot detection methods is due to the turbidity caused from lipoproteins and depend on the reaction's wavelength and direction. Studies report various changes of coagulation tests in lipemic samples but all agree that no reliable results can be obtained on these specimens. METHODS: A 60 years old male was admitted in Emergency Cardiac Care Department due to atrial fibrillation. He didn't refer any other illness and didn't take any drugs. Coagulation tests were ordered as a routine workup prior to anticoagulation therapy. Samples collected at admission, were processed in the laboratory within 30 minutes. PT test was performed in ACL 9000 coagulation analyzer which monitors clot formation by light scatter. TG concentration was measured in Olympus AU640 chemistry analyzer with an enzymatic end point method based on glycerol oxidation. RESULTS: At admission PT was $81.6 \mathrm{sec}(7.48 \%)$, a value discordant to patient's medical history. Plasma looked opalescent in visual inspection, so triglyceride (TG) measurement was ordered. TG level was $314 \mathrm{mg} / \mathrm{dl}$ so we asked the test repetition after 2 hours. The results of the second sample were: PT $12 \mathrm{sec}(116 \%)$ and TG $82 \mathrm{mg} / \mathrm{dl}$. The tests were performed the next morning in fasting samples: PT and TG were within reference ranges.

CONCLUSIONS: Postprandial hypertriglyceridemia is a potential source of preanalytical error in PT test. We suggest the standardized check of lipemic index on coagulation routine tests and the training of emergency nursing staff for preanalytical errors.

Key Words: prothrombin time, postprandial hypertriglyceridemia, preanalytical

\section{HEMOLYSIS INTERFERENCE ON SERUM PROTEINS ZONE CAPILLARY ELECTROPHORESIS}

\author{
V. Vidranski ${ }^{1}$, M. Bušić ${ }^{1}$, M. Njegovan ${ }^{2}$, L. Đerek ${ }^{2}$, I. Vukasović ${ }^{2}$, A. Tešija Kuna ${ }^{2}$ \\ ${ }^{1}$ Department of Oncology and Nuclear Medicine, Sestre milordnice University Hospital Center, Zagreb, Croatia \\ ${ }^{2}$ Department of Clinical Chemistry, Sestre milosrdnice University Hospital Center, Zagreb, Croatia
}

BACKGROUND-AIM: The aim of this study was to evaluate hemolysis interference on serum protein capillary zone electrophoresis performed on analyzer Capillarys2 (Sebia, France). Hemolysis of samples is very common occurrence in laboratory medicine so it is necessary to 
determine interference on electrophoresis testing interpretation because it could decrease $\alpha 2$-fraction and result in appearance of hemoglobin band between $\alpha 1$ and $\alpha 2$ fractions.

METHODS: We used blood from healthy volunteer taken in tube with clot activator and tube with K2EDTA (both Vacuette ${ }^{\circ}$, Greiner Bio-One, Austria). Hemolysis was achieved by subjecting whole blood to half hour freeze-thaw cycle. Ten aliquots of hemolysis-simulated sera prepared by serial dilutions with matched whole blood hemolysis sample were tested. Final serum hemoglobin concentration was within range 0 to $2 \mathrm{~g} / \mathrm{L}(0.01,1.00,1.12,1.24,1.42,1.57,1.60,1.69,1.72$ and 2.00). Hemolysis index (HI) for each aliquot was determined on chemistry analyzer Abbot c8000, (Abbott, Abbott Park, Illinois, USA) and protein fractions were measured on Capillarys2 in duplicate. Acceptance criteria deviation of each fraction was taken from https://www.westgard.com/biodatabase1.htm.

RESULTS: For each electrophoretic fraction, the highest obtained bias in the range of HI up to 2.0 is given in \%, along with acceptable criteria in parenthesis. Biases were as follows: albumin: 2.14 (4.1), alpha1-globulin 3.95 (15.7), alpha2-globulin 11.56 (12.6), beta-globulin 11.58 (11.7) and gamma-globulin 4.36 (16.8).

CONCLUSIONS: Obtained biases did not indicate a clinically significant interference of hemolysis on serum protein capillary electrophoresis up to $\mathrm{HI}$ 2.0, which is also the acceptance limit of $\mathrm{HI}$ for total protein measurement on Abbott c8000 analyzer.

Key Words: pre-analytics, hemolysis, electrophoresis

\title{
HANDLING INDIVIDUALIZED HEMOLYSIS INDICATORS BY MAGNITUDE
}

\author{
$\underline{\text { F. Marques-Garcia }}^{1}$, S. Elena-Perez ${ }^{1}$, D.H. Heredero-Hung ${ }^{1}$ \\ ${ }^{1}$ Clinical Biochemistry Laboratory, Universitary Salamanca Hospital, Salamanca, Spain
}

BACKGROUND-AIM: Interferences are an important source of variability in analytical procedures. One of the most common is the interference produced by hemoglobin, which is mainly related to in vitro processes due to incorrect collection or transport of samples. This interference may cause an increase or decrease in the results of some parameters and this alteration may become clinically relevant. The adequate knowledge of the hemolysis indicators helps us to know better the quality of the extractions with which we work.

METHODS: January-March period 2018, the pre-analytical indicator was evaluated for the follow-up hemolysis in outpatients samples, determined by Hemolyzed Samples / Total Samples`100. Indicator was calculated for total number of samples, setting value of $0.5 \mathrm{~g} / \mathrm{L}$. We had determined the value of clinically significant hemolysis, based on the cut-off point marked by the reference change value (RCV). Above this value, the result was not reported and new extraction is recommended. We selected those parameters whose RCV for hemolysis was less than $0.5 \mathrm{~g} / \mathrm{L}$ (LDH: 56, AST: 265, potassium: 265, folate: 214, and BD: 228), and with them we calculated the indicator itself.

RESULTS: Taking as a cut-off point the value of $0.5 \mathrm{~g} / \mathrm{L}$ in all samples studied, the value of the indicator was $0.1 \%$. When the individual value was calculated for the selected magnitudes when they exceeded the RCV value, the percentages for the indicator were: LDH $2.2 \%$, folate $0.27 \%$, BD $0.97 \%$, AST $0.4 \%$, potassium $0.23 \%$.

CONCLUSIONS: The follow-up of the hemolysis indicators using individual tests offers us a view closer to the real rates of hemolysis that we have in our laboratories, in comparison with the assessment of hemolysis based on a single concentration of hemoglobin for all parameters studied. LDH represents the most appropriate magnitude for the establishment of improvement plans related to the decrease in the number of hemolysed samples.

Key Words: hemolysis, indicator, individualized management

\section{HEMOLYSIS: A PROBLEM THAT EMERGENCY DEPARTMENTS CAN NOT GET RID OF!}

\author{
G. Bozkaya ${ }^{1}$, O. Fenercioglu ${ }^{1}$ \\ ${ }^{1}$ HSU Bozyaka Education and Research Hospital, Department of Medical Biochemistry, Izmir, Turkey
}

BACKGROUND-AIM: Hemolysis is mostly seen in blood samples sent from the emergency department compared to all samples accepted to the laboratory. The tests affected by hemolysis are not analyzed and a new blood sample is requested. The delay in laboratory results causes dissatisfaction both in the physicians and the patients. In this study, it was planned to make a meeting to share knowledge about foremost hemolysis and preanalytical errors with the physicians in emergency department in order to prevent arguments and to give better health care. It was aimed to determine the effect of the meeting by making a survey before and after the meeting.

METHODS: A presentation was made to 40 emergency department doctors in a training meeting in May 2017. Six questions related with these subjects were prepared and 40 doctors were asked to answer them before and after the presentation. The data related to the answers to the questions were evaluated according to the numbers and ratios. Besides, the statistics of hemolyzed specimens were taken from the hospital's information system. 
RESULTS: The question about the causes of hemolysis was fully answered correctly by the $30 \%$ of the participants before the presentation. The ratio increased to $60 \%$ after the presentation. Blood collection with unsuitable devices such as catheters was not known to be a cause of hemolysis by $65 \%$ of the doctors which dropped to $10 \%$ after the presentation. It was determined that hemolysis rate of emergency department compared to all hemolyzed samples were between $47-65 \%$ in the first 7 months period in 2017.

CONCLUSIONS: It is thought that, an awareness about hemolysis was created in emergency department doctors which was defined by some of them as "a problem that emergency departments cannot get rid of" that slow down the patient circulation in emergency department. Similar interactions with clinical departments will help to understand the role of the Medical Biochemists in reliable test results.

Key Words: hemolysis, emergency department, preanalytical factors

\title{
HEMOLYSIS - PREVALENCE AND ECONOMIC BURDEN IN A TERTIARY HOSPITAL
}

\author{
$\underline{\text { C. }}$ Queiroz $^{2}$, Â. Maresch ${ }^{2}$, C. Pereira ${ }^{2}$, T. Rodrigues ${ }^{2}$, R. Monteiro ${ }^{1}$, A. Nabiev ${ }^{2}$, L. Araújo ${ }^{2}$, F. Rodrigues ${ }^{2}$ \\ ${ }^{1}$ Centro Hospitalar do Baixo Vouga, Aveiro, Portugal \\ ${ }^{2}$ Centro Hospitalar e Universitário de Coimbra, Portugal
}

BACKGROUND-AIM: Hemolysis is a leading cause of pre-analytical errors, affecting result reliability and often requiring repeat testing and therefore substantially impacting health care costs and quality. We aimed to evaluate the rate of hemolyzed samples and its economic burden in our hospital.

METHODS: To evaluate hemolysis rates (HR) in 2 settings - wards (W) and emergency department (ED), we calculated the HR in 205,905 samples between 1.12.2017-30.11.2018, from the laboratory information system via assessment of the hemolysis index. The cut-off for the hemolysis categories (mild 60-139, intermediate 140-240 and severe $>240 \mathrm{mg} / \mathrm{dL}$ ) was defined by the manufacturer. Estimates of the economic burden were done via assessment of material costs (needle $€ 0.08$, tube holder $€ 0.08$, collection tube €0.073), personnel costs (value for phlebotomy €0.80) and analytical costs for $\mathrm{K}+€ 1.2$ and LDH€1.3 (chargeable price).

RESULTS: 78450 ED and 127455 W samples were evaluated, with ED rates of 19.9\% $(n=15,650)$ and W rates of 5,3\% (n=6810). There was no statistically significant difference in HR according to gender, age or ED shifts. When calculating the economic burden, we admitted moderate and severely hemolyzed samples as those with criteria to repeat testing of K+ and LDH, totaling 8,134 samples and bringing costs to $€ 28,737.42$. The results of the last HR from our hospital ED in 2013 were of $11.2 \%$ for a total of 85,189 samples.

CONCLUSIONS: In our hospital the nursing staff is in charge of phlebotomy procedures in the W and ED. Still, a considerable difference in HR can be recognized between the 2 settings, denoting additional problems with sampling procedures in the ED that have been aggravated since our 2013 evaluation, requiring better quality practices to be instituted. As for the financial impact, this study despite taking into account only the most frequently ordered analytes in clinical chemistry, potentially underrating the true cost of repeat testing, can elucidate for the cost saving potential of addressing this issue.

Key Words: hemolysis, emergency department, economic burden

\section{TACKLING THE LIPAEMIC SERUM WITH HIGH SPEED CENTRIFUGE: A LABORATORY EXPERIENCE}

$\underline{\text { R. Nanda }}^{1}$, S. Patel ${ }^{1}$, E. Mohapatra ${ }^{1}$

${ }^{1}$ All India Institute of Medical Sciences, Raipur, Chhattisgarh, India

BACKGROUND-AIM: One of the most challenging problem in the preanalytical phase is a lipaemic sample issue.This affects all photometric clinical chemistry and immunoassays that are routinely performed in a laboratory. Ultracentrifugation, the gold standard for lipaemia removal is unavailable in all laboratories.The aim of this study was to assess the utility of high speed cold centrifuge in processing lipaemic samples and reporting them.

METHODS: Analysis of data of all lipaemic samples over a period of last one year wherein a total of five samples were received by the department. Once the samples were received, $1.5 \mathrm{~mL}$ serum was processed in a refrigerated centrifuge, $21130 \times \mathrm{g}$ for 15 minutes (5424 R, Eppendorf). About two-thirds of clear infranatant was transferred into aliquot tubes. This procedure is useful for analytes which are hydrophilic in nature. In our samples, electrolytes were measured by direct ions-selective electrode method. Each sample was run three times and SD and CV\% was calculated. The results of all five patients were matched with the clinical correlation.

RESULTS: The laboratory receives close to 40,000 samples per year out of which five were visibly lipaemic. Of the five patients two were from the paediatric ICU and the rest three from adult ICU. The mean with SD of the electrolytes was calculated. The CV\% for sodium, potassium, and chloride were 2.3, 1.9 and 3.1 respectively. Since each of the samples were from critically ill patients, they were being followed up regularly by the laboratory. The results matched with the clinical findings as well. All patients had an uneventful recovery and were discharged in due course. 
CONCLUSIONS: In the absence of ultracentrifuge, high speed centrifuge can be used for centrifugation of lipaemic serum or plasma, to ensure that a good quality report be given to the patients.

Key Words: lipemia, preanalytical phase, high speed centrifugation

\title{
FACTORS ASSOCIATED WITH HAEMOLYSIS RATES IN ADULT BLOOD SAMPLES IN A UK NATIONAL HEALTH SERVICE TRUST
}

\author{
N.A. Makhumula-Nkhoma ${ }^{2}$, A.M. Batterham ${ }^{1}$, K. Weston ${ }^{1}$, G. Atkinson ${ }^{1}$ \\ ${ }^{1}$ School of Health and Social Care, Teesside University, Middlesbrough, UK \\ ${ }^{2}$ School of Health and Social Care, Teesside University, Middlesbrough, UK; South Tees Hospitals NHS Foundation Trust, Middlesbrough, UK
}

BACKGROUND-AIM: Haemolysed samples may affect patient care, and increase healthcare costs. Awareness of factors that influence haemolysis rates is an important step towards creating quality practices (McCaughey et al., Crit Rev Clin Lab Sci, 2017; 54:59-72). Therefore, we quantified haemolysis rates and explored putative influential factors at three sites; Clinical Decisions Unit (CDU), Acute Assessment Unit (AAU) and Acute Medical Ward (AMU), in a UK National Health Service Trust.

METHODS: Adult biochemistry blood results for 2017 were retrieved from the Trust laboratory information system. A multivariable generalised linear model with a binomial distribution and identity link function was used to explore associations between the proportion (95\% confidence interval, CI) of haemolysed blood samples and clinic location, patient age category, month, day and time of sample collection. Secondary outcomes were duration of transit time from patient to laboratory and laboratory analysis time.

RESULTS: From the 19621 retrieved samples, 359 were excluded due to missing information. The overall haemolysis rate was 11.3 (95\%CI: 10.9-11.8) \%, with the highest site rate of 15 (95\%CI: 13-17) \% in CDU. Rates were particularly high (17-18\%) in CDU in March, April and July, and seemed to peak (24\%) at 15:00 h. Rates were generally highest 17 (95\%CI: 13-20) \% in patients aged $\geq 95 \mathrm{y}$. Differences between month, time of sample collection and transit/analysis times were generally <30 min. The shortest mean transit time of 56 min (95\% CI: $51-62)$ and analysis time of 39 min (95\% CI: 38-40) were in CDU.

CONCLUSIONS: Haemolysis rates were higher for the oldest patients in a UK Health Trust. The large inter-site differences could be explained by differences in staff training between sites. Haemolysis rates of over $20 \%$ were observed at specific sites, and times of year and day. Such information may be useful to help ameliorate these high rates.

Key Words: pre-analytical phase, haemolysis, associations, transit time, analysis time

\section{EVALUATION OF SAMPLE QUALITY FOR COAGULATION ANALYSIS: HIL INDEX}

\author{
$\underline{\text { S. Muhtaroglu }}^{1}$, D. Barlak Keti ${ }^{1}$, M. Karakukcu ${ }^{2}$ \\ ${ }^{1}$ Department of Medical Biochemistry, Erciyes University Faculty of Medicine, Kayseri, Turkey \\ ${ }^{2}$ Department of Pediatric Hematology, Erciyes University Faculty of Medicine, Kayseri, Turkey
}

\begin{abstract}
BACKGROUND-AIM: Haemolysis, icterus and lipaemia (HIL) in specimen may affect the reliability of coagulation test results. This possible interference can be influenced by several factors including the level of interfering substance in plasma, the assay principle and the end-point detection system, that is optical versus mechanical detection. The aim of this study was to determine frequency of HIL in patients' sample for coagulation assays.

METHODS: We assessed 7712 patients' sample over a two month period and determined the incidence of HIL, reling on the manufacturer to document HIL estimates on instrument. Plasma samples were run on CS5100 autoanalyser (Sysmex, Japan), photo-optical clot detection. The instrument identifies HIL specimens with a specific flag. These flags are displayed when the required criteria (for hemoglobin $<40 \mathrm{mg} /$ $\mathrm{dL}$, bilirubin $<2 \mathrm{mg} / \mathrm{dL}$, turbidity $<1300$ formazine turbidity unit) are not met. The quality of the sample (possible existence of hemolysis, and lipemia) is automatically detected with a combination of the multi-wavelength detection method and HIL detector.

RESULTS: The percent of hemolyzed specimens was determined as $1.18 \%$ of all of the samples. We also identified the frequency of lipemia as $0.3 \%$. A total of 533 samples (6.9\%) were icteric. 7 and 8 of samples was rejected due to severe lipemia and hemolysis, respectively.

CONCLUSIONS: The laboratories must monitor and evaluate the quality of the samples and identified problems. Quality results are dependent on quality of specimen. Visual evaluation of the sample is not appropriate because there is significant inaccuracy and inter-individual variation in this type of assessment. HIL may interfere the optical instuments. These interferences can be determined by coagulation analyzer, possessed HIL detection system using multiwavelength light and incorrect results prevented. A test-based interference approach may be useful to avoid unnecessary sample repetition.
\end{abstract}

Key Words: coagulation, hemolysis, icterus, lipemia 


\title{
Sample stability
}

\section{EFFECTS OF DELAYED PROCESSING IN THE SPECIMENS REFERRED TO THE TESTING LABORATORY FROM THE REMOTE CLINICS: IMPACT ANALYSIS OF COST AND PATIENT CARE}

\author{
B. Phiri-Ramongane ${ }^{1}$, A.A. Khine ${ }^{2}$ \\ ${ }^{1}$ Department of Chemical Pathology,Dr George Mukhari Academic Hospital, NHLS \\ ${ }^{2}$ Department of Chemical Pathology,Dr George Mukhari Academic Hospital,NHLS
}

BACKGROUND-AIM: Responding to the shortcomings in the healthcare funding with increasing demand,the national laboratory services in South Africa implemented a rationalised consolidation of laboratory services, which resulted in the remote clinics referring specimens to the testing laboratory in the cities.Clinics serving chronic disease patients were mostly affected due to the high patient numbers,and the impact of test results issued had on medical decisions.The study investigates the impact of logistical delays in the cost of testing and the effects on patient care.

METHODS: A pilot study was performed with thirty-one patients recruited from a local clinic which is 14 km away from the referral laboratory with the requests of serum potassium(K),Alanine Aminotransferase (ALT), and creatinine(CRT).Three specimens were taken from each patient for the study.The first specimen served as a control(no delay in serum separation).The other two samples were delayed for 24 and 48 hours before separation.All specimens were batched and analysed together to avoid inter-run variation.The results of the control and delayed samples were compared for their critical differences,the impact on the patients' management,and the estimation of the cost for testing.

RESULTS: Increased levels of serum K and CRT and low ALT were noted in both the 24 and 48 hour delayed samples.In-vitro haemolysis was more pronounced in the 48 hour delayed samples.Using reference change values significant increases were seen in $96 \%$ of K,3.3\% of ALT and $32.25 \%$ of CRT results at 48 hours.

CONCLUSIONS: The delay in serum separation lead to erroneous results with the following possible consequences repeat testing or drawing fresh sample and retesting;additional tests to confirm the abnormal test;wrong medical decision;delay in medical decision.The findings reveal the hidden cost incurred by the laboratory,clinic and the patient,as well as highlighting the impact on patient care.

Key Words:laboratory services consolidation,delayed processing,patient management,cost of testing.

Key Words: preanalytical phase

\section{PROTOCOL FOR VERIFYING AND DEFINING THE STABILITY LIMIT IN BIOLOGICAL MAGNITUDES OF THE SPANISH SOCIETY OF LABORATORY MEDICINE (SEQC ML)}

\author{
L. Diaz-Garzon $^{1}$, J.M. BauÇa ${ }^{2}$, M. Segovia ${ }^{2}$, D. Martinez ${ }^{2}$, P. Fernandez-Calle ${ }^{1}$, R. Gomez-Rioja ${ }^{2}$ \\ ${ }^{1}$ Analytical Quality Comission, Spanish Society of Laboratory Medicine, Barcelona, Spain \\ ${ }^{2}$ Extra-Analytical Quality Comission, Spanish Society of Laboratory Medicine, Barcelona, Spain
}

BACKGROUND-AIM: The loss of stability of a biological magnitude could be defined as the variation of the property during a defined time period under specific conditions. Whether to consider this variation as significant depends on the specifications for the maximum allowable difference (MAD) set by the laboratory and the stability study design.

METHODS: The Commissions on Analytical and Extraanalytical Quality of the SEQC-ML prepared a guide to evaluate magnitude stability, which includes both a preliminary study to verify a previously defined stability limit (SL), and a more comprehensive study, which allows to calculate the stability equation. Both of them were outlined considering standardized conditions, the use of patient samples instead of healthy volunteers, sample analysis in a single batch (repeatability conditions), and the establishment of MAD according to the models of Milan's consensus. RESULTS: Preliminary study requires to collect 2 samples from at least 3 patients: the basal sample (analyzed in optimal conditions) and the test sample (preserved under defined conditions during a verified defined time). The number of replicates for each sample is calculated with a formula that involves analytical imprecision and MAD. If the relative difference (RD) is lower than the selected MAD in all 3 patients, the SL is considered as verified. Otherwise, the comprehensive study should be performed.

For the comprehensive study, multiple samples from at least 10 patients should be stored under the defined conditions (covering the time range to check) and measured in duplicate. An equation may be outlined by plotting RD over time. SL is assessed by interpolation of the MAD specification, considering confidence intervals.

CONCLUSIONS: We propose a robust and standardized method to verify (preliminary study) or to define (comprehensive study) the stability limit under defined conditions. Harmonization in stability studies could help in the transferability of stability limit among different laboratories. 


\title{
NORWEGIAN STABILITY PROJECT
}

\author{
G.B.B. Kristensen ${ }^{1}$, M.C. Tollånes ${ }^{1}$ \\ ${ }^{1}$ Norwegian Quality Improvement of Laboratory Examinations (Noklus), Norway
}

BACKGROUND-AIM: The Norwegian stability project was established in 2014. Its aims were to prepare a freely available Norwegian stability protocol and to establish a national database for results of stability testing.

METHODS: A working group coordinated by Noklus (Norwegian Quality Improvement of Laboratory Examinations) with representatives from BFI (Institute for Biomedical Laboratory Scientists) and NSMB (Norwegian Society for Medical Biochemistry) was established in 2014. The group prepared a protocol describing two recommended models for conducting stability studies: (i) "the Batch-method", suitable for analytes that are known to be stable under certain storage conditions, and (ii) "the Pants-method", suitable when it is not known whether the material is stable during storage, e.g. hematological samples. A prerequisite for both methods is that the quality requirements for bias and total allowable error are known. Noklus set up a website and a database to share information, published online in 2015.

RESULTS: As of nov-2018, 33 completed stability studies were registered in the database with detailed and standardized information on the conduction of the experiments, results and conclusions, including a link to the raw data. The Batch-method was most commonly used. The protocol and database were evaluated by Norwegian participating laboratories in March 2018. Participants strongly recommended implementation of a process of verification of results prior to publication.

CONCLUSIONS: The stability protocol and database are considered useful to participants. Harmonization, sharing of knowledge and reduction of unnecessary workload and costs are key components. The project is currently expanding to include all Nordic countries.

Key Words: stability, temperature, duration time

\section{EFFECT OF STORAGE TIME AND TEMPERATURE ON URINE CHEMISTRY VALUES}

\author{
E. Zhuravleva $^{1}$, I. Torshina ${ }^{1}$, E. Khasyanova ${ }^{1}$, E. Chashchikhina ${ }^{1}$, N. Igonina ${ }^{1}$, E. Kondrasheva ${ }^{1}$, A. Salikhova ${ }^{1}$ \\ ${ }^{1}$ LLC Independent Laboratory INVITRO, Moscow, Russia
}

BACKGROUND-AIM: Compliance with the storage and transportation requirements ensures analyte stability in the sample. Most samples are delivered to our laboratory within $24 \mathrm{~h}$ to $72 \mathrm{~h}$. Aim of this work was to evaluate of storage time and temperature effects on some urine chemistry values and to compare with manufacturer's claims.

METHODS: The urine samples were collected from 5 healthy volunteers. Magnesium, phosphorus (after acidification), uric acid (after alkalization) and glucose were measured (Architect c8000, Abbott) within $4 \mathrm{~h}$ from sampling (Day 0), after storage at $4{ }^{\circ} \mathrm{C}$ for $24 \mathrm{~h}, 48 \mathrm{~h}$ and $72 \mathrm{~h}$ and at $-20^{\circ} \mathrm{C}$ for $72 \mathrm{~h}$. Average biases from Day 0 results were compared with Westgard acceptable biases for magnesium, phosphorus, uric acid and reference change value (RCV) for glucose (no biological variation data). Statistical significance was estimated by Wilcoxon test (Statistica, StatSoft).

RESULTS: Magnesium remained stable through the whole period (average biases of $0.96 \%, 1.97 \%, 0.60 \%$ at $4{ }^{\circ} \mathrm{C}$ for $24 \mathrm{~h}, 48 \mathrm{~h}$ and $72 \mathrm{~h}$ respectively; $0.09 \%$ at $-20^{\circ} \mathrm{C}$ for $72 \mathrm{~h}, \mathrm{p}>0.05$ ) that consistent with manufacturer's claims. For phosphorus and uric acid, the manufacturer does not recommend urine sample storage at $4{ }^{\circ} \mathrm{C}$ and $-20{ }^{\circ} \mathrm{C}$. However, phosphorus and uric acid results did not change significantly at $4{ }^{\circ} \mathrm{C}$ for $24 \mathrm{~h}$ (average biases of $0.11 \%$ and $0.85 \%$ respectively, $\mathrm{p}>0.05$ ). Significant changes occurred after storage over $24 \mathrm{~h}$ ( $\mathrm{p}=0.043$ for both tests), but within the acceptable limits of biases. Glucose level didn’t change significantly (average biases of $4.70 \%, 1.03 \%, 3.80 \%$ at $4{ }^{\circ} \mathrm{C}$ for $24 \mathrm{~h}, 48 \mathrm{~h}$ and $72 \mathrm{~h}$ respectively; $0.77 \%$ at $-20^{\circ} \mathrm{C}$ for $72 \mathrm{~h}, \mathrm{p}>0.05$ ) but exceeded manufacturer's limits. The observed relative average biases were within the acceptable biases and RCV of these analytes at all conditions (time and temperature).

CONCLUSIONS: The obtained results were used to establish the pre-analytical requirements for urine storage at $4{ }^{\circ} \mathrm{C}$ for 48 hours and at $-20{ }^{\circ} \mathrm{C}$ for 72 hours.

Key Words: sample storage, urine, pre-analytical phase

\section{STABILITY OF REFRIGERATED WHOLE BLOOD SAMPLES FOR OSMOTIC FRAGILITY TEST}

\author{
G.L. Salvagno ${ }^{1}$, D. Demonte ${ }^{1}$, F. Dima ${ }^{1}$, G. Lippi ${ }^{1}$ \\ ${ }^{1}$ Section of Clinical Biochemistry, University Hospital of Verona, Verona, Italy
}

BACKGROUND-AIM: Osmotic fragility test (OFT) is conventionally used to measure erythrocyte resistance to hemolysis, so assisting the diagnosis of thalassemia and hereditary spherocytosis, which are two relatively frequent erythrocytes disorders. This test is manual and time-consuming, and is not performed daily in most clinical laboratories, so that whole blood samples may be stored for days before delayed testing. This study was aimed at defining the stability of whole blood samples used for assessing erythrocyte osmotic resistance.

METHODS: Twenty-one consecutive routine whole blood samples, collected into $5.4 \mathrm{mg}$ K2EDTA tubes (Vacutest Kima, Padova, Italy) and referred to the local laboratory for assessment of erythrocyte osmotic resistance, were tested immediately after collection (day 0 ) and at 
different time intervals afterward (day 1, 2, 3, 4, 7, 10 and 14). During the study period all samples were kept refrigerated at $4{ }^{\circ} \mathrm{C}$. The OFT was performed with Osmored Monotest (1.3\% glycerol; Eurospital, Trieste, Italy), which uses both hyperosmotic (normal value, $>70 \%$ ) and hyposmotic (normal value, $<30 \%$ ) solutions. Results at different time points were compared with those obtained at day 0 .

RESULTS: The mean value of hyperosmolar and hyposmolar resistance ( $\%$ hemolysis) were $73 \pm 34 \%$ and $21 \pm 18 \%$ at day $0,73 \pm 31 \%$ ( $p=0.488$ ) and $23 \pm 18 \%(\mathrm{p}<0.001)$ at day $1,73 \pm 31 \%(\mathrm{p}=0.425)$ and $25 \pm 19 \%(\mathrm{p}<0.001)$ at day $2,72 \pm 30 \%(\mathrm{p}=0.409)$ and $25 \pm 18 \%(\mathrm{p}<0.001)$ at day 3 , $73 \pm 30 \%(\mathrm{p}=0.414)$ and $26 \pm 19 \%(\mathrm{p}<0.001)$ at day $4,79 \pm 28 \%(\mathrm{p}=0.031)$ and $30 \pm 25 \%(\mathrm{p}<0.001)$ at day $7,85 \pm 22 \%(\mathrm{p}=0.002)$ and $45 \pm 19 \%$ $(\mathrm{p}<0.001)$ at day 10, $90 \pm 17 \%(\mathrm{p}<001)$ and $53 \pm 18 \%(\mathrm{p}<0.001)$ at day 14 , respectively. The median relative increase of values was larger than the reference change value (i.e., 33\%) at day 3 for hyposmolar resistance, but this limit was never reached for hyperosmolar resistance. CONCLUSIONS: Sample stability seems critical for OFT. Whole blood specimens should not be stored refrigerated for $>2$ days at $4{ }^{\circ} \mathrm{C}$ before testing.

Key Words: osmotic fragility test, sample stability, preanalytical phase

\title{
INFLUENCE OF PRE-ANALYTICAL ERRORS ON THE DETERMINATION OF TSH CONCENTRATIONS IN NEWBORNS
}

\author{
$\underline{\text { A. Stojnić }}^{1}$ \\ ${ }^{1}$ Institute of Laboratory Diagnostic, University Clinical Centre of the Republic of Srpska, Banja Luka, Bosnia and Hercegovina
}

BACKGROUND-AIM: Determination of the concentration value of Thyroid Stimulating Hormone (TSH) from the sample of dried drops of capillary blood on filter paper taken in all newborn infants, belongs to the neonatal screening program implemented throughout the territory of the Republic of Srpska (RS). As the cards are transported from the maternity wards across RS to the laboratory in which the analysis is carried out, the aim was to verify that the irregular transport or the exposure of the cards during transport to high temperatures could affect the TSH concentration values.

METHODS: Firstly, the values of TSH concentration are determined after the material is received in the laboratory $(\mathrm{n}=100)$, then the samples are heated for 48 hours at a temperature of $30^{\circ} \mathrm{C}$, after which the TSH concentration is again measured, and all the measurements are repeated at the temperatures of $40^{\circ} \mathrm{C}$ and $50^{\circ} \mathrm{C}$. All measurements are made in triplicate.

RESULTS: Variance analysis with one factor of variability is done to estimate the effect of temperature tolerance on the value of TSH concentration using the SPSS 10 program. The differences of the arithmetic averages, for different measurement temperatures, relative to Tukey's statistics, show the level of significance of 0,05. A singlifical difference is determined between the values of TSH concentrations at temperatures of: $24^{\circ} \mathrm{C}$ and $40^{\circ} \mathrm{C}, 24^{\circ} \mathrm{C}$ and $50^{\circ} \mathrm{C}, 30^{\circ} \mathrm{C}$ and $50{ }^{\circ} \mathrm{C}, 40^{\circ} \mathrm{C}$ and $24^{\circ} \mathrm{C}, 40^{\circ} \mathrm{C}$ and $50^{\circ} \mathrm{C}, 50^{\circ} \mathrm{C}$ and $24^{\circ} \mathrm{C}, 50^{\circ} \mathrm{C}$ and $30^{\circ} \mathrm{C}, 50^{\circ} \mathrm{C}$ and $40{ }^{\circ} \mathrm{C}$. CONCLUSIONS: Based on the obtained results, it can be concluded that with the increase in temperature, the TSH concentration values are increased, so that at a temperature of $50{ }^{\circ} \mathrm{C}$ this dependence is established for all three measurements. The indicated dependence can lead to false positive results in borderline cases, as well as an increase in the number of results found in the grey area, and thus to unnecessary re-sampling. As this is about sensitive population, it is necessary to reduce the pre-analytical errors to a minimum.

Key Words: TSH, temperature, preanalytical error

\section{THE IMPACT OF OBSERVATIONAL TIMING ON THE PREANALYTICAL STABILITY OF ADRENOCORTICOTROPIC HORMONE (ACTH)}

\author{
N. Bekaia ${ }^{1}$, M. Akhvlediani ${ }^{1}$, L. Kiphiani ${ }^{1}$ \\ ${ }^{1}$ Research Institute of Clinical Medicine, Tbilisi, Georgia
}

BACKGROUND-AIM: Adrenocorticotropic hormone (ACTH) is sensitive to temperature and observation timing. The pre-analytical stability of ACTH was carried out in two groups of patients; one cohort of patients comprised of healthy volunteers, whilst another cohort included patients with renal failure and Adison disease. The impact of observational timing and the significance of plasma separation from formed elements after centrifugation was assessed.

METHODS: During the three month a total of 24 patients were analyzed using Electrochemiluminescent immune assay (ECL), One cohort of patients included 10 volunteers, whilst another the total of 14 patients: 9 patients with renal failure and 5 patients with Adison disease. Two blood samples were obtained from both cohort of patients. Specimens were immediately centrifuged after blood collection. The pre-cooled sampling tubes were used. The samples were analyzed at two points: immediately and after 2 hours of storage at $22 \mathrm{C}$ temperature. Centrifuged blood samples (12 sample) was kept in K EDTA sampling tubes, whilst some plasma (12 samples) was moved into clean tubes. The content of ACTH was measured in both type of tubes - immediately and after two hours.

RESULTS: The concentration level of ACTH kept in K ETDA plastic tube has decreased between initial measurement and measurement taken $2 \mathrm{~h}$ post centrifugation by $2.5 \%$.On the other hand, neither time nor temperature had any effect on hormone concentration levels kept in clean tubes. The difference in change between these two groups was $15 \%$. 
CONCLUSIONS: Preanalytical stability of ACTH is affected by observational period after centrifugation and separation of aliquots within $\mathrm{K}_{3}$ EDTA plastic tubes post centrifugation.

Key Words: ACTH, stability, centrifugation, $\mathrm{K}_{3}$ EDTA tubes, measuring time

\title{
PRIMARY HEALTHCARE CENTERS: STABILITY POTASSIUM
}

$\underline{\text { C. Gomez Gomez }}{ }^{1}$, M. Dolade Botias ${ }^{1}$, M.A. Huertas Contreras ${ }^{1}$, F. Moreno Flores ${ }^{1}$, J. Minchinela Girona ${ }^{1}$, M.A. Llopis Diaz ${ }^{1}$

${ }^{1}$ Clinical Core Laboratory, Laboratorio Clínico Metropolitana Nord, Badalona, Spain

BACKGROUND-AIM: The stability limit of an analyte in a biological sample can be defined as the time required until a measured property acquires a bias higher than a defined specification. In our laboratory samples are transported from 117 in primary healthcare centers (PHC) to the main laboratory through different routes under controlled temperature and transport time. Blood samples are centrifugated when arriving to the laboratory. The aim of this study was to assess the stability of potassium considering our routine transport conditions.

METHODS: A retrospective analysis was made with data obtained from one month, in samples from 13 PHC of different routes and from the blood collection area of our laboratory. For each center the mean of potassium concentrations was calculated and compared between centers. At the same time, in order to asses if the time until centrifugation affects the potassium concentration, an experiment was performed in the laboratory, collecting two samples from 15 healthy volunteers. One sample was centrifugated and analyzed after 1 hour of collection and the other after 3 hours. Blood collection was made in BD Vacutainer ® SST 5 mL containers with gel and all samples were analyzed in AU5800 (Beckman Coulter $\left.{ }^{\circledR}\right)$.

RESULTS: There were not temperature differences between centers, but a significant increase of potassium concentration mean was observed when time until centrifugation increased $(p<0.05)$. This last fact was also observed in the laboratory experiment $(p<0.0001)$.

CONCLUSIONS: An increase of potassium concentration was observed in the study when centrifugation is delayed. To prevent this increase, probably the centrifugations should be reduced by reducing the transport time or by providing centrifuges to PHC. Moreover, in specifically situations, patients can be derived to the laboratory blood collection area.

Key Words: preanalytical phase

\section{STABILIZATION OF GLUCOSE CONCENTRATION IN THE NEW VACUETTE® FC MIX BLOOD COLLECTION TUBE FOR DIAGNOSIS OF DIABETES}

\author{
$\underline{\text { S. Griebenow }}^{1}$, J. Racek ${ }^{2}$ \\ ${ }^{1}$ Greiner Bio-One GmbH, Kremsmuenster, Austria \\ ${ }^{2}$ Institute of Clinical Biochemistry and Hematology, Pilsen, Czech Republic
}

BACKGROUND-AIM: Reliable glucose test results are required in case of prolonged transport times of blood collection tubes. The use of tubes with an additive composed of citrate, EDTA and NaF to effectively stabilize glucose levels is recommended by German Diabetes Association. The aim was to show the long-term stability of initial glucose concentration in specimens spun directly after collection and compared to whole blood specimens stored at room temperature.

METHODS: A study was done at Faculty Hospital (Pilsen) using VENOSAFE FC Mixture vs. VACUETTE FC Mix tubes. Altogether, 50 healthy subjects and 50 diabetics were recruited after giving IC. Venous blood was drawn into four tubes (two tubes each tube type). One tube of each type was spun directly after collection and the second after whole blood storage for $48 \mathrm{~h}$ at RT. Following collection, plasma was measured immediately after centrifugation to obtain initial values (fasting) and after $48 \mathrm{~h}$ for evaluation of glucose stability on a COBAS c702 (Roche: Hexokinase method). Statistical evaluation was done by STATISTICA.

RESULTS: Evaluation of all clinical results for glucose was done on basis of maximal allowed deviation for a single value (glucose $11 \%$ : guideline of German Association of Laboratory Testing (Rilibäk)). The utilization of both tubes did not reveal any clinically nor statistically significant deviations $(\mathrm{p}<0.05)$. The values resulted in an initial highest deviation of $5.7 \%$ (diabetics), and after $48 \mathrm{~h}$ of $7.3 \%$ (healthy). Comparable highest deviations for initial values in relation to $48 \mathrm{~h}$ values were obtained for VENOSAFE (7.5\%), and VACUETTE tubes (7.7\%). The storage of whole blood specimens for $48 \mathrm{~h}$ showed no significant deviation.

CONCLUSIONS: The FC Mix tube is suitable for reliable determination of blood glucose, one of the most frequently measured analytes and of primary importance in diagnosis, monitoring and diabetes therapy. Stability of glucose in whole blood specimens stored up to $48 \mathrm{~h}$ at RT has been proven.

Key Words: diabetes, glycolysis, glucose stabilization 


\title{
STABILITY OF FAECAL CALPROTECTIN EXTRACTS
}

\author{
$\underline{\text { A. Vrtaric }}^{1}$, M. Bozovic ${ }^{1}$, N. Nikolac Gabaj ${ }^{1}$, J. Culej ${ }^{1}$, L. Milevoj Kopcinovic ${ }^{1}$, I. Herceg ${ }^{2}$ \\ ${ }^{1}$ University Department of Chemistry, Medical School University Hospital Sestre Milosrdnice, Zagreb, Croatia \\ ${ }^{2}$ Medical biochemistry laboratory, General hospital "Dr. Tomislav Bardek”, Koprivnica, Croatia
}

BACKGROUND-AIM: The BÜHLMANN CALEX® Cap (BÜHLMANN Laboratories AG, Switzerland) is a single use tube filled with extraction buffer used for extraction of calprotectin (fCal) from human stool samples. According to manufacturer's specifications, fCal extract is stable 6 days at $2-8^{\circ} \mathrm{C}$. The aim of this study was to verify whether the manufacturer's criteria are met and test if fCal extracts are stable if stored for a longer period (i.e. for 10 days at $2-8^{\circ} \mathrm{C}$ ).

METHODS: Faecal calprotectin was extracted from $\mathrm{N}=10$ stool samples according to the manufacturer's specified protocol. Extracts were then aliquoted in 6 aliquots; initial fCal was measured on the day of extraction, while the remaining aliquots were stored at $2-8{ }^{\circ} \mathrm{C}$ for 1,3 , 5, 7 and 10 days, respectively. Measurements were made on the Abbott Architect c8000 (Abbott Laboratories, Abbott Park, USA) using BÜHLMANN fCAL® turbo reagent (BÜHLMANN Laboratories AG, Switzerland). Bias from the initial fCal concentration was calculated for each individual sample. Mean bias was calculated for each storage period and compared to Instand criteria which is $36 \%$. Statistical analysis was performed using Medcalc (Ostend, Belgium) Friedman ANOVA test. P-value was set at $<0.05$.

RESULTS: The initial fCal concentration expressed as median (interquartile range) was 198.8 (58.7-731.9) mg/kg. Median concentrations measured in extracts stored for 1, 3, 5, 7 and 10 days were 213.5 (62.4-716.3), 204.5 (54.4-690.3), 207.0 (51.2-682.4), 205.0 (53.7-679.5) and 189.3 (56.2-657.6) $\mathrm{mg} / \mathrm{kg}$, respectively. Bias from the initial fCal concentration for extracts stored for 1, 3, 5, 7 and 10 days were $-3.52 ;-4.41 ;-0.26 ;-7.03$ and $-3.41 \%$, respectively. No difference between the initial fCal and fCal measured in extracts stored in the observed time periods was found ( $\mathrm{P}=0.830$ ). CONCLUSIONS: Faecal calprotectin extract stability met the criteria specified by the manufacturer. We confirmed that fCal extracts might be stored for periods up to 10 days at $2-8^{\circ} \mathrm{C}$.

Key Words: faecal calprotectin, extraction, stool

\section{STABILITY OF SAMPLES USED FOR APTT TEST. A PRACTICAL APPROACH.}

\author{
${\underline{\text { C. } \text { Marzo }^{2}}}^{2}$ X. Clotet $^{2}$, X. Gómez-Arbonés ${ }^{1}$, A. Ferrero ${ }^{2}$, M. Casals ${ }^{2}$, M. Bernal ${ }^{2}$, Y. Rico ${ }^{2}$, M. Ibarz ${ }^{2}$ \\ ${ }^{1}$ IRBLleida, Lleida, Spain. \\ ${ }^{2}$ Laboratory Medicine Department, University Hospital Arnau de Vilanova, IRBLleida, Lleida, Spain.
}

BACKGROUND-AIM: Consolidation of laboratories has led to the reception of a large number of samples in a short time. The aim of this study was to assess the stability of samples used for PTTA tests in the real conditions of our laboratory.

METHODS: 244 samples from in and outpatients were analyzed. Blood collection was carried out by the laboratory staff following the current procedure. Becton Dickinson (BD) Vacutainer ${ }^{\mathrm{TM}}$ blood collection tubes were used (ref 363079). Centrifugation complied with BD's recommendations. All samples were maintained at room temperature $\left(22-25^{\circ} \mathrm{C}\right)$ and protected from light. The instrument used was ACL-TOP $700 \mathrm{CTS}$ (ILInstrumentation Laboratory). The results of the initial Activated Partial Thromboplastin Time APTT (s) and Activated Partial Thromboplastin Time ratio ( $\mathrm{r}$-APTT ) were compared with those obtained after 4 hours storage. The differences were compared to the acceptable limits derived from the biological variation. All data were analyzed with the Statistical Package SPSS (SPSS Inc., Chicago, IL, USA).

RESULTS: The mean initial APTT was $31.04 \mathrm{~s}$ and the mean initial APTT was 1.020; the final APTT was $31.25 \mathrm{~s}$ and the final $\mathrm{r}$ - APTT was 1.026. We found an excellent correlation between the initial results and at 4 hours of the APTT ( $R=0,979$, regression line slope 0,943; IC95\% 0,918$0,968)$ and $r$ - APTT $(R=0,978$ regression line slope 0,940; IC95\% 0,914-0,965, although a statistically significant lengthening of both APTT ( $p<$ $0.001)$ and $\mathrm{r}$ - APTT $(\mathrm{p}<0.001)$ was observed. The mean differences between the final and initial APTT values were $0.20 \mathrm{~s}(95 \% \mathrm{CI} 0.05-0.36)$ and those of the r- APTT were 0.064 (95\% CI 0.001-0.012).

CONCLUSIONS: Statistically significant differences were observed between the results obtained at 4 hours vs baseline. Although not clinically significant (lower than the desirable total error based on individual biological variation) it is considered appropriate to continue, whenever possible, with the immediate processing of the samples.

Key Words: stability, coagulation, storage

\section{PREANALYTICAL STABILITY OF CLINICALLY RELEVANT CHEMISTRY ANALYTES IN SEROUS FLUIDS}

\author{
L. Milevoj Kopcinovic ${ }^{1}$, M. Brcic ${ }^{1}$, A. Vrtaric ${ }^{1}$, J. Culej ${ }^{1}$, M. Bozovic ${ }^{1}$, A. Topic ${ }^{1}$, M. Miler ${ }^{1}$, N. Nikolac Gabaj ${ }^{1}$ \\ ${ }^{1}$ Department of Clinical Chemistry, Sestre milosrdnice University Hospital Centre, Zagreb, Croatia
}

BACKGROUND-AIM: Preanalytical stability for analytes in serous fluids (SF) is largely unknown. Our aim was to evaluate stability of clinically relevant analytes in SF (i.e. pleural and peritoneal effusions) stored under different conditions prior to analysis. 
METHODS: Leftovers from 20 consecutive SF samples initially referred to our laboratory for routine analysis were separated. Baseline values for total proteins (TP), albumin (ALB), lactate dehydrogenase (LD), cholesterol (CHOL), triglycerides (TRIG), creatinine (CREA), urea, glucose, amylase (AMY) and C-reactive protein (CRP) were measured within 1 hour from sample receipt. Leftover SF samples were then aliquoted in 5 aliquots and stored for 6 hours at room temperature (RT), and 3, 7, 14 and 30 days at $-20^{\circ} \mathrm{C}$. At the end of each storage period all analytes were measured. Analytes were measured using the Abbott Architect c8000 (Abbott Laboratories, Abbott Park, USA). Mean bias was calculated for each parameter and respective storage period, and compared to stability limits (SL) set according to CROQALM criteria. Statistical analysis was performed using Medcalc (Ostend, Belgium). $\mathrm{P}<0.05$ was considered statistically significant.

RESULTS: Significant differences comparing all storage periods were observed for LD, CREA, glucose and CRP (P <0.001). Calculated biases for TP, ALB, CHOL, TRIG, CREA, urea, AMY and CRP in all storage periods tested were below the SL. Glucose and LD were stable for 6 hours at RT, with biases of -3.8 and $0.2 \%$, respectively. Biases exceeding the SL were observed for glucose and LD in SF when stored for 3, 7, 14 and 30 days at $-20{ }^{\circ} \mathrm{C}$ (i.e. $-7.6,-11.5,-17.5$ and $-27.5 \%$ for glucose, and $-17.1,-25.5,-30.1$ and $-40.3 \%$ for $\mathrm{LD}$, respectively).

CONCLUSIONS: Our results indicate that TP, ALB, CHOL, TRIG, CREA, urea, AMY and CRP may be safely analysed when SF samples are stored up to 6 hours at RT and/or 30 days at $-20^{\circ} \mathrm{C}$. However, serous fluid glucose and LD are stable only up to 6 hours stored at RT.

Key Words: preanalytical phase, stability, pleural fluid, peritoneal fluid

\title{
EFFECT OF A CHEMICAL PRESERVATIVE ON URINE SAMPLES FOR URINALYSIS
}

\author{
L.M. Vargas Morales ${ }^{1}$, P. Hernández Morales ${ }^{1}$, A. Bárcenas Morales ${ }^{1}$, B. Jurado Manzano ${ }^{1}$, A. Rodríguez Rodríguez ${ }^{1}$ \\ ${ }^{1}$ Facultad de Ciencias Químicas, Universidad Autónoma de San Luis Potosí, México
}

BACKGROUND-AIM: Errors in the preanalytic phase of urinalysis can alter the analytes of interest and even destroy them, the use or not of a chemical preservative could influence the analytical phase. The aim of the study is to analyze if the preanalytical factor "use of chemical preservative" significantly influences the chemical study of urine.

METHODS: Thirteen samples of urine samples (first morning urine) were included in the analytical study. The BD Vacutainer® Urinalysis Cup Kit was used to collect the sample, which includes a cup to obtain the sample and a conical tube with sodium propionate $94 \%$, ethyl paraben $5.6 \%$ and chlorhexidine $0.4 \%$ as preservative. Patients were asked to transfer urine to the tube with a preservative after collecting the urine sample, and the remaining sample from the cup was also delivered to the laboratory, which was considered as a urine sample without conservator. The standardized chemical analysis was performed on the urine samples with Multistix® 10 SG test strips and the automated reading system CLINITEK Advantus, both from SIEMENS, was used; the maximum analysis time of two hours after the collection of the urine sample was considered. For the statistical analysis the Wilcoxon signed rank test was used, the statistical package used is the software SPSS Statistics ${ }^{\circledR} 20$, the established statistical significance was a value of $\mathrm{p} \leq 0.05$.

RESULTS: A significant difference was observed in specific gravity $(Z=-2.121, p=0.034)$ and no significant differences were observed in the rest of the parameters of the chemical study of the urine.

CONCLUSIONS: The decreased specific gravity could accelerate the lysis of some microscopic elements such as cells and microorganisms after collection; the use of a chemical preservative prevents changes in the specific gravity of urine samples analyzed in less than two hours.

Key Words: preanalytic phase, urinalysis, chemical preservative

\section{ASSESSMENT OF THE STABILITY OF TWO CONTROVERSIAL MAGNITUDES: TOTAL BILIRUBIN AND ETHANOL}

\author{
P. Argente Del Castillo Rodríguez ${ }^{1}$, M.A. Ballesteros Vizoso ${ }^{1}$, M.M. Parera Roselló ${ }^{1}$, M. Pastor García ${ }^{1}$, A.R. Pons Mas ${ }^{1}$, J.M. Bauça ${ }^{1}$ \\ ${ }^{1}$ Department of Laboratory Medicine, Hospital Universitari Son Espases, Palma de Mallorca, Spain
}

BACKGROUND-AIM: The stability of a magnitude depends on several parameters, including storage temperature, humidity, exposure to light or contact with air. Its evaluation may improve patient's safety and comfort, as further blood drawings may not be required. Our aim was to evaluate the stability of two magnitudes with a high request number in the clinical laboratory: total bilirubin (TB) and ethanol.

METHODS: Prospective observational study. We randomly collected 93 samples with TB $>1.2 \mathrm{mg} / \mathrm{dL}$ and classified them into: low (1,2-5mg/dL) and high $(>5 \mathrm{mg} / \mathrm{dL})$. TB was quantified in the same sample at different intervals: $0,2,8$ and $24 \mathrm{~h}$. We also took 36 samples with ethanol $>1 \mathrm{~g} / \mathrm{L}$ and 4 samples with values $<1 \mathrm{~g} / \mathrm{L}$. Determinations were made at 0, 2, 4 and $8 \mathrm{~h}$.To reproduce the actual working conditions, all samples were stored at room temperature, uncapped and unprotected from light. All determinations were made on the Architect c16200 platform (Abbott). For the establishment of the stability limit (SL), average and StDev of the values in the different times were calculated and the following total error specifications were considered: TB 13.5\% (from SEQC-ML) and ethanol 17\% (from CLIA).

RESULTS: For low TB values, $(1.2-5 \mathrm{mg} / \mathrm{dL}, \mathrm{N}=74)$, an average variation of $0.01 \%,-2.02 \%$ and $-3.91 \%$ at 2,8 and $24 \mathrm{~h}$ was observed. For high TB values $(>5 \mathrm{mg} / \mathrm{dL}, \mathrm{N}=18$ ) we observed an average variation of $-0.10 \%,-1.91 \%$ and $-3.46 \%$ at 2,8 and $24 \mathrm{~h}$. No significant differences were seen between them. SL was set at $24 \mathrm{~h}$ regardless of TB concentration. Ethanol is stable at both low and high levels for $8 \mathrm{~h}$ (variation $-12.3 \%$ ) since the moment of sample uncapping. Samples with low ethanol are slightly more stable than higher concentrations. 
CONCLUSIONS: The demonstrated stability of TB (24h) and ethanol ( $8 \mathrm{~h})$ lead to an improvement in patient safety, mainly by reducing the samples needed to be collected. This highlights the relevance of the participation of the laboratory in the knowledge of the stability of each parameter.

Key Words: stability, ethanol, bilirubin

\title{
A COMPARISON OF STABILITY OF CHEMICAL ANALYTES IN PLASMA FROM THE BD VACUTAINER B BARRICORTM TUBE WITH MECHANICAL SEPARATOR VERSUS TUBES CONTAINING GEL SEPARATOR
}

\author{
G. Gawria ${ }^{1}$, L. Tillmar ${ }^{1}$, E. Landberg ${ }^{1}$ \\ ${ }_{1}^{1}$ Department of Clinical Chemistry and Department of Clinical and Experimental Medicine, Linköping University, Linköping, Sweden
}

BACKGROUND-AIM: There is a need of prolonged stability of certain chemical analytes in lithium heparin tubes with separators. A new tube with a mechanical separator has recently been launched (Barricor ${ }^{\mathrm{TM}}$ ). According to the manufacturer, the cell count in plasma is reduced after centrifugation of this tube, which therefore may decrease leakage and affect stability of substances with high intracellular concentration.

METHODS: Samples from 122 patients were analysed after storage at $4^{\circ} \mathrm{C}$ for 3 to 80 hours. Tubes were transported manually ( $\mathrm{n}=20$ ) or by car $(n=102)$. Blood was collected in vacuum tubes containing lithium heparin with two different types of separators; gel separator (Vacutainer ${ }^{\circledR}$ PST ${ }^{\mathrm{TM}}$, Becton Dickinson and Vacuette ${ }^{\circledR}$, Greiner bio-one) and mechanical separator (Vacutainer ${ }^{\circledR}$ Barricor ${ }^{\mathrm{TM}}$, Becton Dickinson). All tubes had similar aspiration volume and were centrifuged at similar time and force. Seven analytes were measured by Cobas c701 (Roche).

RESULTS: The Barricor tube showed increased stability of phosphate and potassium and similar stability of aspartate aminotransferase, glucose, homocysteine, lactate dehydrogenase and magnesium compared with gel tubes. Maximal allowable bias for phosphate was exceeded after $70 \mathrm{~h}$ for Barricor tubes compared with $29 \mathrm{~h}$ for gel tubes and for potassium after $38 \mathrm{~h}$ for Barricor tubes versus $8 \mathrm{~h}$ for gel tubes. Transportation did not affect stability. Haemolysis index was slightly lower in Barricor tubes than in gel tubes $(\mathrm{p}=0.01)$.

CONCLUSIONS: Implementing the new Barricor tube will improve stability of potassium and phosphate in plasma. Blood sampling facilities far from the laboratory may benefit from using these tubes, thus diminishing preanalytical errors.

Key Words: stability, tube, separator, potassium, phosphate

\section{EVALUATION OF THE EFFECT OF LONG-TERM STORAGE AT -20 $0^{\circ}$ ON SAMPLE STABILITY IN BD VACUTAINER® BARRICORTM BLOOD COLLECTION TUBES}

\author{
$\underline{\text { K. Ford }}^{1}$, S. Church ${ }^{1}$ \\ ${ }^{1}$ Becton Dickinson UK Ltd.
}

BACKGROUND-AIM: BD Vacutainer ${ }^{\circledR}$ Barricor ${ }^{\mathrm{TM}}$ was introduced to the market in March 2016. As part of the post-market studies to provide data to end-users, analyte stability in plasma collected in BD Barricor ${ }^{\mathrm{TM}}$ tubes following prolonged storage (1 or 12 months) in the primary tube at $-20{ }^{\circ} \mathrm{C}$ was evaluated. Visual observations of the samples were recorded and barrier integrity assessed. A plasma gel separator tube (BD Vacutainer ${ }^{\circledR}$ PST ${ }^{\mathrm{TM}} \mathrm{II}$ ) was stored under the same conditions to identify differences in analyte stability due to the plasma matrix rather than temperature effects on the primary tube.

METHODS: This study compared plasma analytes for equivalence following storage at $-20{ }^{\circ} \mathrm{C}$ in primary tubes for up to $12 \mathrm{months}$. Six tubes were collected from each of 40 subjects, 3 each of BD Barricor ${ }^{\mathrm{TM}} 3.0 \mathrm{~mL}$ and BD PST ${ }^{\mathrm{TM}} \mathrm{II}$ 3.0mL. Tubes were mixed and centrifuged under recommended conditions before visual assessment prior to analysis for a range of chemistry and immunochemistry analytes. Tubes were frozen at $-20^{\circ} \mathrm{C}$ and defrosted and visually assessed after 1 and 12 months before testing for the same analytes.

RESULTS: No significant difference in analyte stability was seen between PST ${ }^{\mathrm{TM}}$ II and BD Barricor ${ }^{\mathrm{TM}}$ primary tubes. In some cases the bias was less marked in the BD Barricor ${ }^{\mathrm{TM}}$ tube compared against BD PST ${ }^{\mathrm{TM}} \mathrm{II}$ (e.g. Lactate BD Barricor ${ }^{\mathrm{TM}}$ at $12 \mathrm{months}(2.13 \pm 0.34 \mathrm{mmol} / \mathrm{L}) \mathrm{vs}$. Initial time $(2.04 \pm 0.38 \mathrm{mmol} / \mathrm{L}), \mathrm{p}=0.326$; BD PST ${ }^{\mathrm{TM}} \mathrm{II}$ at $12 \mathrm{months}(2.26 \pm 0.38 \mathrm{mmol} / \mathrm{L}) \mathrm{vs}$. Initial time $(2.11 \pm 0.40 \mathrm{mmol} / \mathrm{L}, \mathrm{p}=0.000 . \mathrm{LDH}$ BD Barricor ${ }^{\mathrm{TM}}$ at 12 months $(271 \pm 49 \mathrm{U} / \mathrm{L})$ vs. Initial time $(162 \pm 25 \mathrm{U} / \mathrm{L}), \mathrm{p}=0.002$; BD PST ${ }^{\mathrm{TM}}$ II at 12 months (328 $\left.\pm 58 \mathrm{U} / \mathrm{L}\right)$ vs. Initial time $(160 \pm 28 \mathrm{U} / \mathrm{L}, \mathrm{p}=0.000))$.

CONCLUSIONS: Plasma frozen in the BD Barricor ${ }^{\mathrm{TM}}$ primary tube has comparable or sometimes better stability to plasma frozen in the BD PST $^{\text {TM }}$ II tube up to 12 months.

Key Words: preanalytical phase 


\title{
STABILITY TESTING OF HEPARIN ANTI XA ACTIVITIY IN PLASMA
}

\author{
L. Omazić ${ }^{2}$, M. Milić ${ }^{1}$, V. Šerić ${ }^{1}$ \\ ${ }^{1}$ Department of clinical laboratory diagnostics, Osijek University Hospital and Faculty of Medicine, Josip Juraj Strossmayer University of Osijek, \\ Osijek, Croatia \\ ${ }^{2}$ Department of laboratory and transfusion medicine, County General Hospital Vukovar and Croatian Veterans' Hospital and Faculty of Medicine, \\ Josip Juraj Strossmayer University of Osijek, Osijek, Croatia
}

BACKGROUND-AIM: Heparins are anticoagulants used to treat or prevent thromboembolic diseases. For monitoring patients on LMWHs or $\mathrm{UFH}$, the plasma anti-Xa assay is used. Prompt sample processing within 1 hour is required to avoid heparin neutralization by platelet factor 4. Sample processing after 1 hour can lead to underestimation of anti-Xa i.e. heparin. The aim of this study is to determine the effect of storage duration on anti-Xa activity using plasma left on cells.

METHODS: Blood samples were taken from 10 patients receiving LMWH therapy 4 hours after the last injection. Samples were collected in 3.2\% trisodium citrate anticoagulant BD Vacutainer tubes and centrifuged at 2500g for 20 minutes. Colorimetric assay Sta-Liquid anti-Xa on STA-R Evolution, Stago Diagnostica was used to measure anti-Xa activity. Anti-Xa activity was determined in plasma samples left on cells 1st, 2nd and 3rd hour after blood was drawn. Statistical analysis was performed using MedCalc 12.4.0.0 software (MedCalc, Mariakerke, Belgium). Friedman test was done to determine level of significance between every time point. Wilcoxon singed-rank test was used to determine level of significance between 1st hour and 2nd or 3rd hour. Level of significance was set at $\mathrm{P}<0.05$.

RESULTS: Friedman test for paired samples showed that there are no statistical differences between the levels of anti-Xa activity in 1st, 2nd and 3rd hour after blood samples were collected $(\mathrm{P}=0,731)$. Wilcoxon test showed that there are no statistical differences between 1 st and $2 \mathrm{nd}$ hour $(\mathrm{P}=0,375)$ and 1st and 3rd hour $(\mathrm{P}=0,301)$.

CONCLUSIONS: Despite general recommendation to test plasma samples for anti Xa activity within 1 hour, this study showed that plasma left on cells can be tested for anti-Xa activity up to 3 hours.

Key Words: heparin, anti-Xa, heparin monitoring, thromboembolic diseases

\section{INFLUENCE OF STORAGE TIME TO THE TESTING OF BLOOD GASES AND ACID BASE STATUS IN PREANALYTICAL PHASE}

\author{
$\underline{\text { I. Zhilenkova }}^{1}$, P. Borisova ${ }^{1}$, D. Dalinkina ${ }^{1}$, V.V. Slepysheva ${ }^{1}$, A.V. Kozlov ${ }^{1}$ \\ ${ }^{1}$ The North-West State Medical University n. a. I. I. Mechnikov, Saint-Petersburg
}

BACKGROUND-AIM: The aim of present study was to estimate influence of time storage of Blood Gases and Acid Base status results during preanalytical phase.

METHODS: Arterial blood samples of 20 patients from intensive care were taken into heparinized syringes and measured in ABL800 FLEX (Radiometer, Denmark) after collection, than after $10 \mathrm{~min}, 20 \mathrm{~min}, 30 \mathrm{~min}$ and $40 \mathrm{~min}$. Samples were stored at room temperature. Results of Blood Gases and Acid Base status were compared with estimation of relative difference of the values between initial and each subsequent points. RESULTS: $\mathrm{pH}$ changed no more than $0.15 \%$ in all patients ( $\mathrm{p}>0.05)$ during $40 \mathrm{~min}$ of storage. Relative difference of p02 values after 10 min became 4.5\% ( $p>0.05)$, after $20 \mathrm{~min}-9.5 \%$ ( $\mathrm{p}<0.05)$, after $30 \mathrm{~min}-11 \%(\mathrm{p}<0.05)$ and after $40 \mathrm{~min}-9.8 \%(\mathrm{p}<0.05)$. The bigger differences of p02 values were observed in patients with initially high levels of this parameter. Relative difference of pCO2 values during storage time was not significant ( $p>0.05)$ in period of 10 and 20 min after sample collection $(1.3 \%$ and $1.7 \%$ ), and results became significantly different $(\mathrm{p}<0.05)$ after 30 and $40 \mathrm{~min}(2.7 \%$ and $3.8 \%)$. The Glucose concentration was decreased in all samples during of time storage with nonsignificant difference after $10 \mathrm{~min}-1.1 \%$ ( $\mathrm{p}>0.05$ ), and became significantly ( $\mathrm{p}<0.05$ ) after $20 \mathrm{~min}-2.5 \%, 30 \mathrm{~min}-3.5 \%$ and $40 \mathrm{~min}-4 \%$. The Lactate values were increased in all samples. Relative difference of Lactate results compared with initial values were $10.8 \%$ after 10 min ( $\mathrm{p}<0.05$ ), 16.1\% after $20 \mathrm{~min}$ ( $\mathrm{p}<0.05), 24.9 \%$ after $30 \mathrm{~min}(\mathrm{p}<0.05), 33.3 \%$ after $40 \mathrm{~min}(\mathrm{p}<0.05)$.

CONCLUSIONS: Performing the analysis of Blood Gases and Acid Base Status no more than 10 minutes after sample collection allows obtaining the results that has no influence to test interpretation. Influence of storage time especially for p02, pCO2, Glucose and Lactate could be explained by the continued metabolism in cells after sample collection.

Key Words: blood gases, acid base, preanalytical phase

\section{STABILTY OF METHOTREXATE IN BD SSTII BLOOD COLLECTION TUBES WITH SERUM SEPERATOR}

M.F. Schaffer ${ }^{1}$, P. Neyer ${ }^{1}$, A. Hammerer-Lercher ${ }^{1}$, L. Bernasconi ${ }^{1}$

${ }^{1}$ Institute of Laboratory Medicine, Kantonsspital Aarau, Aarau, Switzerland 
BACKGROUND-AIM: Methotrexate is widely used as chemotherapeutic and immunosuppressive agent. Accurate measurement of concentration in serum is important for Therapeutic Drug Monitoring (TDM). Many laboratories use serum gel separator blood collection tubes which are often stored at $4^{\circ} \mathrm{C}$ until analysis. The stability of 14 monitored drugs after storage in serum separator tubes SST II from Becton-Dickinson was previously reported [1], however the effect of storage on blood samples containing Methotrexate as not been verified.

[1] V. Bush, J. Blennerhasset, A. Wells, A. Dasgupta, Ther Drug Monit. 23:259-262 (2001)

METHODS: In this study the long-term stability (24 hours) of Methotrexate after storage at $4{ }^{\circ} \mathrm{C}$ in Serum Separator tubes SST II (Becton-Dickinson) was investigated. The separator gel is based on acrylic resin. Methotrexate measurements (Architect i2000, Abbott) were performed on consecutive routine serum samples with different drug concentrations. Pearson's coefficient of determination, Passing-Bablok regression and Bland-Altman difference plot were used to compare the results.

RESULTS: The comparison of the Methotrexate concentration in serum before and after storage resulted in a coefficient (Pearson) of r2 $=0.9866$ and relative Bland-Altman bias of $0.4 \%$. The corresponding Passing-Bablok regression slope was 1.00.

CONCLUSIONS: The results indicate that the gel barrier had no significant effect on the concentration of Methotrexate. This suggests that serum separator tubes SST II of Becton-Dickinson are suitable for therapeutic drug monitoring of Methotrexate even after long-term storage (24 hours).

Key Words: gel separator tubes, therapeutic drug monitoring, stability, blood specimen collection

\title{
OPTIMISING CENTRIFUGATION OF SERUM SAMPLES ON 39 CLINICAL CHEMISTRY ANALYTES
}

\author{
M. Bratuša ${ }^{1}$, R. Kogovšek ${ }^{1}$, S. Mikec ${ }^{1}$, P. Meško Brguljan ${ }^{1}$ \\ ${ }^{1}$ Clinical Chemistry Department, University Clinic Golnik, Golnik, Slovenia
}

BACKGROUND-AIM: Centrifugation is an important preanalytical step, which crucially influence TAT. Recommendations for centrifugation procedures are not uniform. Aim of this study was to verify the suitability of optimized centrifugation procedure with the goal to reduce TAT. METHODS: Paired 89 venous blood samples were collected into serum separator tubes BD Vacutainer® SST ${ }^{\mathrm{TM}}$ II Advance Tubes. Currently used (2300xg, $15 \mathrm{~min}, 25^{\circ} \mathrm{C}$, acceleration 9, breaking 9) and new optimised (3000xg, $5 \mathrm{~min}, 25^{\circ} \mathrm{C}$, acceleration 7, breaking 9) centrifugation procedure were used (Heraeus Megafuge 16R, Thermo Scientific). Samples were analysed on a Cobas ${ }^{\circledR}$ c6000 (Roche Diagnostics). Measured data were statistically evaluated using Medcalc software (V.18.2.1). The Spearman's rank correlation coefficients were calculated and the Wilcoxon paired samples test was performed. Additionally, the parameters with statistically significant differences were plotted on the BlandAltman plot. Maximum allowed difference between both methods was determined according to biological variation criteria.

RESULTS: Non-normal distribution of parameter groups was shown with the Shapiro-Wilk's test (63/78). Spearman's rank correlation coefficients displayed a very good association between the variables (rho 0,90-1,00). Statistically significant differences $(\mathrm{P}<0,05)$ were found for: (-Amylase, Chloride, CRP, (C)-GT and Glucose, with the Wilcoxon test. Differences were evaluated based on Bland-Altman plot for clinical relevance. Obtained differences $(0,27 \% ;-0,38 \% ; 0,29 \% ; 0,42 \% ; 0,93 \%)$ were compared against Bias from desirable Ricos criteria on biological variation $(7,4 \% ; 0,5 \% ; 21,8 \% ; 11,1 \% ; 2,3 \%)$. All five results from Bland-Altman plot agreed with selected criteria.

CONCLUSIONS: Based on our results we can conclude, that proposed centrifugation procedure does not affect patient results and is suitable for routine use. With the use of optimised centrifugation procedure TAT could be reduced for at least 10 minutes.

Key Words: centrifugation, preanalytics, TAT

\section{INVESTIGATION OF THE STABILITY AND ACTIVITY OF $\alpha$-AMYLASE IN WATER DILUTED HUMAN SALIVARY SAMPLES IN FORENSIC MEDICINE}

\author{
N. Portnova ${ }^{2}$, V. Sidorov ${ }^{2}$, L. Khorovskaya ${ }^{1}$, I. Loban ${ }^{1}$ \\ ${ }^{1}$ North-Western State Medical University named after I.I. Mechnikov, Saint-Petersburg, Russia. \\ ${ }^{2}$ Saint-Petersburg State Healthcare Institution Bureau of Forensic Medical Examination, Saint-Petersburg, Russia
}

BACKGROUND-AIM: Saliva identification in spots of material evidence is widely used for diagnostic purpose in Forensic Medicine. The aim of this study was to investigate stability and activity of $\alpha$-amylase in water diluted salivary samples depending on time storage.

METHODS: Human saliva taken from 2 volunteers into special sterile container was diluted using deionized water with $\mathrm{pH}=7.4 \mathrm{in}$ low concentration 1:10, middle concentration 1:50, high concentration 1:80. Concentration of $\alpha$-amylase was measured 5 times during 5 hours ( 1 workday) in 5 replicates per each series by kinetic colorimetric method on the microplate reader «SUNRISE» (Tecan, Austria). Samples were stores in room temperature $\left(+18-20^{\circ} \mathrm{C}\right)$. The average, within- and between series imprecision and percentage of lost $\alpha$-amylase activity were calculated.

RESULTS: The within series CV\% was in about 1.07-3.41\% for high, $0.30-1.48 \%$ for middle and $0.87-1.22 \%$ for low concentrations of $\alpha$-amylase. Between series imprecision during 1 workday was 3.9\% for high, $7.5 \%$ for middle and $7.9 \%$ for low concentrations. By the end of the workday 
activity of $\alpha$-amylase decreased to $81.6 \%$ in low, $82.8 \%$ in middle and $90.8 \%$ in high concentrations that demonstrated activity lost up to $9.2 \%$ $17.2 \%-18.4 \%$ respectively.

CONCLUSIONS: $\alpha$-amylase activity in water solutions of human salivary samples was significantly decreased during 5 hours of storage time for low and middle concentrations of saliva $(\mathrm{p}<0.05)$ that could affect test interpretation and give erroneous results. Thus investigation of $\alpha$-amylase in different concentrations of water solutions demonstrated accepted stability for 1 workday for correct forensic investigation on material evidence.

Key Words: stability, saliva, material evidence, $\alpha$-amylase

\title{
INVESTIGATING THE EFFECT OF PNEUMATIC TUBE TRANSPORT AFTER SAMPLE CENTRIFUGATION ON GENERAL BIOCHEMISTRY SAMPLES COLLECTED IN BARRICORTM TUBES
}

\author{
$\underline{\text { S. Coward }}^{1}$, L. Mcadam ${ }^{1}$, L. Reilly ${ }^{1}$, F. O'neill ${ }^{1}$, S. Lavery ${ }^{1}$, R. Loughlin ${ }^{1}$, G. Mckeeman ${ }^{1}$ \\ ${ }^{1}$ Clinical Biochemistry, Royal Victoria Hospital, Belfast, Northern Ireland
}

BACKGROUND-AIM: Barricor ${ }^{\mathrm{TM}}$ tubes have demonstrated quick sample separation, whilst also providing a clean plasma sample with a mechanical barrier between the plasma and the cells. We examined the beneficial and detrimental effects of centrifuging Barricor ${ }^{\mathrm{TM}}$ samples at source, prior to sending them to the laboratory via a pneumatic chute.

METHODS: A lab staff member was embedded within the Emergency Department of the Royal Victoria Hospital, Belfast. 1. Barricor ${ }^{\mathrm{TM}}$ samples were processed at source (LIMS entry and centrifugation at $4000 \mathrm{~g}$ for $3 \mathrm{mins}$ ). Samples were then sent via the pneumatic chute system to the laboratory, and analysed for the requested tests (Roche c701 analysers). The following day, plasma from these samples was aliquoted, re-spun at 4000g ( 3 mins) and re-analysed for the same tests. Data were then compared. 2. Paired Barricor ${ }^{\mathrm{TM}}$ samples were taken. One was processed at source, as in (1), and then sent to the laboratory along with the second (uncentrifuged) sample. The second sample was then centrifuged in the laboratory by the same method ( $4000 \mathrm{~g}, 3 \mathrm{mins}$ ) and both samples processed for the same (requested) tests. Data were then compared.

RESULTS: 1 . Significantly (but not clinically) different results $(\mathrm{p}<0.05)$ were seen for 10 of 20 analytes, though study design may have contributed to some of these. Significant changes to all three indices values $(p<0.05)$ were also seen, with the lipaemic (turbidity) index showing the greatest changes. 2. Significantly (but not clinically) different results $(\mathrm{p}<0.05)$ were seen for 2 of 10 analytes. Significant changes to all three indices values $(p<0.05)$ were also seen. Similar changes to the lipaemic (turbidity) index were seen, as in $(1)$, but changes to the haemolytic index were greater than (1).

CONCLUSIONS: There was no significant negative clinical impact on biochemistry results when samples were transported after centrifugation. This approach improves sample quality, and hence result quality, with respect to haemolysis.

Key Words: pre-analytics, centrifugation, pneumatic transport system, haemolysis

\section{EVALUATION OF SEVEN ROUTINE BIOCHEMICAL ANALYTES STABILITY IN BD BARRICOR\& BD SST TUBE \& VACUSERA SST TUBE}

\author{
$\underline{\text { E. Avci }}^{1}$, R. Nar ${ }^{1}$, K. Akpinar ${ }^{1}$, S. Demir ${ }^{1}$ \\ ${ }^{1}$ Medical Biochemistry Department, Faculty of Medicine, Pamukkale University, Denizli, Turkey
}

BACKGROUND-AIM: Clinical laboratories mostly used serum separating tubes for analyzing routine biochemistry analytes.Relatively inert gel used in these tubes is affecting analyte concentrations.Becton, Dickinson Company (BD) has recently developed the BD Barricor ${ }^{\mathrm{TM}}$ plasma blood collection tube, which has a mechanical separator.According to the manufacturer, the quality of plasma is improved, the centrifugation time is shorter and post centrifugation analyte concentrations are more stable in samples that have been stored in the primary tube.In present study,we compared seven routine analyte stability by using BD Barricor, SST tube and Vacusera SST Tube

METHODS: Sixty volunteers venous blood samples were drawn into three tubes.Randomization, mixing tubes, filling volume, 30-minute clotting time for serum tubes, and centrifuge conditions were considered during observation. Hemolyzed samples were excluded. Glucose, urea, creatinine, lactate dehydrogenase (LDH), Sodium (Na), Chloride (Cl) and Potassium (K) levels analyzed at 0, 4, 8 and 12 hours on Cobas 8000 (Roche Hitachi)analysers. Internal quality control were performed for biochemistry parameters. Bias results were calculated and revealed as bias\%.All bias results were compared to clinical acceptance limits (CAL) parameters those taken from CLIA sites. SPSS version 24 package and $R$ package program were used for statistical analyses

RESULTS: Analytical CV results for glucose, LDH, creatinine, urea, $\mathrm{Na}, \mathrm{K}$, and $\mathrm{Cl}$ were $3.22 \%, 5.8 \%, 5.28 \%, 3.9 \%, 2 \%, 1.95 \%, 1.37 \%$ respectively.All analytes showed good accordance with each other in terms of stability monitoring. CAL limits for chloride, creatinine, glucose, potassium, lactate dehydrogenase and sodium are $3 \mathrm{mmol} / \mathrm{L}, 0.3 \mathrm{mg} / \mathrm{dl}, 10 \%, 0,5 \mathrm{mmol} / \mathrm{L}, 20 \%$ and $3 \mathrm{mmol} / \mathrm{L}$ respectively. At 12 . hour glucose showed a difference between LH tube-BD SST\&LH Tube-Vacusera SST respectively 10,16\&11,14 
CONCLUSIONS: By using Barricor ${ }^{\mathrm{TM}}$ tubes and prompt centrifugation, analysis for seven analytes could be performed within $12 \mathrm{~h}$ of specimen stability.

Key Words: comparison, tube comparison, sample stability, preanalytical phase

\title{
SHORT (4 HOURS) AND LONG-TERM (4 WEEKS) STABILITY OF PROTHROMBIN TIME (PT), ACTIVATED PARTIAL THROMBOPLASTIN TIME (APTT) AND FIBRINOGEN (FBG) IN CITRATE PLASMA SAMPLES
}

\author{
I. Kuktić ${ }^{1}, \underline{\text { V. Radišić Biljak }}{ }^{1}$, AM. Šimundić ${ }^{1}$ \\ ${ }^{1}$ University Hospital „Sveti Duh“, Department of Medical Laboratory Diagnostics, Zagreb, Croatia
}

BACKGROUND-AIM: Manufacturer (Siemens, Germany) states that PT is stable in citrate plasma up to $24 \mathrm{~h}$ at room temperature (RT), whereas aPTT and Fbg should be determined within $4 \mathrm{~h}$ after sample collection. While long-term storage (for up to 2 weeks) at $\leq-20{ }^{\circ} \mathrm{C}$ is declared for aPTT, data for PT and Fbg are not provided by the manufacturer. The aim of this study was to verify manufacturer's claims for sample stability for PT, aPTT and Fbg.

METHODS: We measured PT (Innovin), aPTT (Actin FS) and Fbg (Multifibren U) in 20 citrate plasma samples (baseline results) on BCS XP coagulation analyzer (Siemens, Germany). Plasma samples were kept in a primary tube at RT and measurements were repeated after $4 \mathrm{~h}$ and $24 \mathrm{~h}$. Two plasma aliquots were stored at $\leq-20^{\circ} \mathrm{C}$ and measurements were repeated after 2 and 4 weeks. Statistical analysis was performed using the MedCalc Statistical Software version 16.2.0 (MedCalc Software bvba, Ostend, Belgium). The results were tested with Friedman test. $\mathrm{P}<0.5$ was considered significant. CROQALM (Croatian Quality Assessment in Laboratory Medicine) criteria were used as acceptable bias: $\pm 15 \%$ for PT, $\pm 7 \%$ for aPTT and $\pm 14 \%$ for Fbg.

RESULTS: Bias for PT at $4 \mathrm{~h}$ and $24 \mathrm{~h}$ at RT was not statistically significant $(\mathrm{P}=0.382)$. Clinically significant bias for $\mathrm{PT}$ was observed in $5 / 20$ samples stored for $24 \mathrm{~h}$. During $24 \mathrm{~h}$ storage at RT, aPTT and Fbg values differed statistically $(\mathrm{P}<0.001)$, but not clinically. There was no clinically significant difference for PT and Fbg in samples stored up to 4 weeks at $-20^{\circ} \mathrm{C}$. However, we observed both clinically and statistically significant difference in aPTT values $(\mathrm{P}<0.001)$ stored for 2 and 4 weeks at $-20^{\circ} \mathrm{C}$

CONCLUSIONS: We have confirmed manufacturer's claims for PT, aPTT and Fbg short-term stability (up to 4h) at RT in primary tubes. Our findings for long-term stability of aPTT contradict manufacturer's claims. While not declared by the manufacturer, long-term stability at $-20{ }^{\circ} \mathrm{C}$ for PT and Fbg was acceptable up to 4 weeks.

Key Words: preanalytical phase, plasma stability, manufacturer's claims, coagulation

\section{LEAKING NASOPHARYNGEAL ASPIRATES DURING TRANSIT: THE IMPACT OF A SAFETY ALERT AND IMPORTANCE OF PROPER LABORATORY SPECIMEN TRANSPORTATION}

\author{
W. Musabaike $^{1}$, T. Rockenbach ${ }^{1}$, Y.C. Wu ${ }^{1}$, M. Nakrani $^{1}$, C. Morris ${ }^{1}$ \\ ${ }^{1}$ Great Ormond Street Hospital for Children, London, UK
}

BACKGROUND-AIM: Nasopharyngeal aspirates (NPA) are commonly collected for prompt diagnosis of viral respiratory infections. At Great Ormond Street Hospital for Children, NPA samples were either hand-delivered to the laboratory or transported via the pneumatic tube system (or chute). In 2016, there was a high number of leaking NPA in the chute system due to the use of containers unapproved for the chute. This caused diagnostic delays not only only for the patients affected but to other patients because the chute system had to be shut down for decontamination. On 24 March 2017, a safety alert was issued and communicated which stated that all NPA samples must be delivered by hand only. This study examined the impact of the safety alert, which was part of a wider Quality Improvement project aimed at improving the pre-analytical process.

METHODS: Data for NPA samples received between 01/03/2016 - 30/04/2018 were extracted from the Laboratory Information System. The number of leaking NPA samples and the sample transport time before and after the release of the safety alert were compared.

RESULTS: There was a 65\% drop in the number of leaking NPA samples after the issue of the safety alert. There was no significant change in the average transport time before and after the the issuance of the safety alert.

CONCLUSIONS: The safety alert was effective in reducing the number of leaking NPA samples received in the laboratory with minimal impact on sample transport time. Until a new sample container that is approved to be used in the chute system can be found, the safety alert will remain in effect. This study illustrates the importance of proper sample transport as failings can affect not only patient outcomes but also the healthcare and portering teams that are involved in the sample processing pathway.

Key Words: transport, leakage, pneumatic 


\title{
SHORT-TERM STABILITY OF INSULIN IN SAMPLES FROM DIFFERENT BLOOD COLLECTION TUBES
}

\author{
M. Krhač ${ }^{1}$, K. Grdiša ${ }^{2}$, M. Vučić Lovrenčić ${ }^{1}$ \\ ${ }^{1}$ Clinical department of medical biochemistry and laboratory medicine, Merkur University Hospital, Zagreb \\ ${ }^{2}$ Department of laboratory diagnostics, General County Hospital Požega, Požega
}

BACKGROUND-AIM: Measurement of the levels of blood insulin, a hormone responsible for the blood glucose regulation, may be a useful tool for the assessment of insulin resistance in type 2 diabetes and metabolic syndrome management. However, sample type together with overall stability is of great importance because insulin is easily degraded by circulating peptidases. Previous reports indicate that concentration remains stable in EDTA plasma, but not serum samples for 24 hours. The aim of our study was to compare insulin levels in two sample types, recommended plasma from EDTA-containing tubes (BD ${ }^{\mathrm{TM}}$ VacutainerTM K3EDTA Tubes,USA) and a serum from tubes with a new generation of gel separator (BD Vacutainer ${ }^{\mathrm{TM}} \mathrm{SST}^{\mathrm{TM}}, \mathrm{USA}$ ). Additionally, we assessed insulin stability in both sample types within 24 hour storage at room temperature and $+4^{\circ} \mathrm{C}$.

METHODS: Venus blood was sampled from 18 volunteers parallel in SST-serum tubes and EDTA-containing tubes. After centrifugation, analysis was done immediately and after 24 hour storage in different temperature conditions (room temperature and $+4^{\circ} \mathrm{C}$ ). Plasma samples were transferred in secondary tubes while the serum separation was obtained by gel in SST-tubes. Insulin was measured on ADVIA Centaur-XP analytical system (Siemens Healthineers, USA).

RESULTS: Passing Bablok regression analysis revealed proportional differences for insulin concentration in fresh samples from SST-serum compared to EDTA-plasma tubes ( $\mathrm{y}=-3,1115(95 \% \mathrm{CI}=-18,4947$ to 3,5230$)+1,1531(95 \% \mathrm{CI}=1,0228$ to 1,3646$) \mathrm{x})$. Regarding sample stability, insulin levels in SST-serum samples were found to be significantly lower in both samples stored at room temperature and $+4^{\circ} \mathrm{C}(\mathrm{P}=0,0235)$, while EDTA-plasma samples obtained satisfactory stability ( $\mathrm{P}=0,6599$;Kruskall Wallis ANOVA).

CONCLUSIONS: SST-serum tubes could not preserve insulin from degradation for 24 hour period regardless of the storage temperature.

Key Words: insulin stability, EDTA-plasma, SST-serum gel separator tubes

\section{SERUM OSMOLALITY: SHORT (8 HOURS) AND LONG TERM (1 MONTH) SAMPLE STABILITY}

\author{
$\underline{\text { A. Đuras }}^{1}$, V. Radišić Biljak ${ }^{2}$, I. Ostroški ${ }^{1}$, AM. Šimundić ${ }^{2}$ \\ ${ }^{1}$ General Hospital "Varaždin”, Department of Medical Biochemistry, Varaždin, Croatia \\ ${ }^{2}$ University Hospital "Sveti Duh”, Department of Medical Laboratory Diagnostics, Zagreb, Croatia
}

BACKGROUND-AIM: Changes in osmolality (hyper- and hypo-osmolality) are associated with increased morbidity and mortality. However, stability of serum for measurement of this critical analyte is unknown. Our aim was to investigate the short- (8 hours) and long-term (1 month) stability of serum osmolality.

METHODS: Blood samples from 40 inpatients were collected in clot-activator tubes (Becton, Dickinson and Company, Franklin Lakes, USA) and centrifuged at $3220 \mathrm{~g}$ for $10 \mathrm{~min}$ (Hettich, Kirchlengern, Germany). Serum osmolality was measured in triplicate on OSMOMAT 3000 (Gonotech, Berlin, Germany) analyzer and mean values of the repeated measurements were used in the analysis. Primary tubes were left at room temperature (RT) for $8 \mathrm{~h}$, and aliquots were stored at $-20^{\circ} \mathrm{C}$ for 1 month. Statistical analysis was performed using the MedCalc Statistical Software version 16.2.0 (MedCalc Software bvba, Ostend, Belgium). The results were tested with Friedman test. P value $<0.5$ was considered significant. Deviations were assessed against reference change value (RCV) of 5.13\%, as acceptance criterion.

RESULTS: Median osmolalities (and interquartile range, IQR) were as follows: 295 (292.5-301) for baseline values, 294 (290-297.5) and 292 (288.5-296) for $8 \mathrm{~h}$ at RT and $1 \mathrm{month}$ at $-20^{\circ} \mathrm{C}$, (in mOsmol $/ \mathrm{kg}$ ) respectively. Method CV was $1.1 \%$. There was a statistically significant difference in osmolality concentration measured after 8 hours at RT and after one month at $-20{ }^{\circ} \mathrm{C}$, compared to baseline results $(\mathrm{P}<0.001)$. Deviations from baseline osmolality were within acceptance criteria, as follows: $0.6 \%$ (95\% CI: 0.3-0.8) and 1.4\% (95\% CI: 0.8-2.1), for 8h at RT and after 1 month at $-20^{\circ} \mathrm{C}$.

CONCLUSIONS: Serum samples for osmolality measurement are stable if kept in primary tubes at RT up to 8h, or if stored aliquoted up to 1 month at $-20^{\circ} \mathrm{C}$. This allows that osmolality is requested as add-on or reflex test during the same day, or even within 1 month (e.g. to be shipped to a reference laboratory).

Key Words: preanalytical phase, osmolality, sample stability

\section{STABILITY OF EXTERNAL QUALITY (EQ) SAMPLES FOR FAECAL OCCULT BLOOD - COMPARISON OF IMMUNOCHROMATOGRAFIC AND GUAIAC TESTS}

\author{
$\underline{\text { I. Taradi }}^{1}$, E. Matišić ${ }^{1}$, S. Perkov ${ }^{1}$, M.M. Kardum Paro ${ }^{1}$ \\ ${ }^{1}$ Department of Medical Biochemistry and Laboratory Medicine, Merkur University Hospital, Zagreb, Croatia
}


BACKGROUND-AIM: Immunochromatografic and guaiac-based faecal occult blood tests are used worldwide in population screening for colorectal cancer. Guaiac-based tests reveal hem component of hemoglobin whilst immunochromatografic tests reveal globin component of hemoglobin. Hem component is more stable than globin. However, immunochromatografic tests have higher sensitivity. The aim of this study was to examine EQ samples stability for faecal occult blood of both tests.

METHODS: Three EQ samples (two liquid artificials S001 and S002 and a solid one S003) for faecal occult blood (immunochromatografic and guaiac- based tests) were examined every other day during 10 days. Samples were handled just as patients samples following the organizers and test instructions and analysis was done immediately after their arrival to the laboratory. Appearance of the blue color in guaiac test and pink line in immunochromatografic test field revealed positive result.

RESULTS: Liquid artificials S001 and S003 were positive and a solid S002 was negative for faecal occult blood of both tests. Blue color in quaiac- based test as well as pink line in immunochromatografic test field was of the same intensity during the 10 days of examination for samples S001 and S003.

CONCLUSIONS: External quality samples for faecal occult blood were stable after opening during 10 days of the examination and gave reliable results regardless of the used test. Although immunochromatografic test has better sensitivity it also has satisfying stability compared to guaiac test.

Key Words: EQA, stability, faecal occult blood, guaiac test

\title{
INTERMEDIATE STORAGE PARTIALLY AFFECTS STABILITY OF ROUTINE ANALYTES FOR CLINICAL STUDIES AND BIOBANKING
}

\author{
$\underline{\text { N. Sédille-Mostafaie }}^{2}$, C. Schild ${ }^{2}$, D. Aebischer ${ }^{1}$, E. Florence ${ }^{1}$, A. Leichtle ${ }^{2}$ \\ ${ }^{1}$ Center of Laboratory Medicine, Inselspital, Bern University Hospital, and University of Bern, Bern, Switzerland \\ ${ }^{2}$ University Institute of Clinical Chemistry, Inselspital, Bern University Hospital, and University of Bern, Bern, Switzerland
}

BACKGROUND-AIM: Specimen collection, processing, receiving, and retrieval are key processes for biobank sample quality that have recently been implemented in the latest ISBER guidelines. We investigated whether high-throughput processing and intermediate storage in a fully automated clinical chemistry lab are sufficient to maintain the quality of routine analytes for clinical studies and biobanking.

METHODS: We randomly collected 20 patients' leftover sera after routine analysis. From each sample, three aliquots from the top to the bottom layer of a tube were collected on days 1, 3, and 5. A Roche ${ }^{\mathrm{TM}}$ Cobas 8000 analyser was used to measure the serum concentration of 18 selected routine analytes. During these 5 days, the samples were refrigerated at $4{ }^{\circ} \mathrm{C}$. We used generalised linear mixed-effects modelling with analyte serum concentrations as dependent, storage days and layers and their interaction as fixed, and patient pseudo-ID as random effects.

RESULTS: We found small effects of storage day for potassium and protein, lipase, HDL, and sodium. After multiple testing correction, only potassium and protein remained significant. The levels of all other tested analytes remain stable when automatically refrigerated for up to 5 days in clinical routine. Even for sedimentation-prone analytes (e.g. lipids, protein), analyte levels did not significantly differ between layers. CONCLUSIONS: We conclude that processing and up to 5 days intermediate storage on a total lab automation system is sufficient to maintain the quality of the aforementioned routine analytes for clinical studies.

Key Words: preanalytics, biobanking, stablity, refrigeration

\section{ERYTHROCITE SEDIMENTATION RATE SAMPLE STABILITY}

\author{
$\underline{\text { R. Mihaylov }}^{2}$, P. Blagovesta ${ }^{1}$, D. Stoeva ${ }^{1}$, R. Petkova ${ }^{1}$, N. Marinova ${ }^{1}$ \\ "SMDL - Ramus" Ltd., Sofia, Bulgaria \\ 2“SMDL - Ramus” Ltd., MC „Y. Filaretova“, Sofia, Bulgaria
}

BACKGROUND-AIM: The ESR test is one of the most commonly used tests in the clinical laboratory practice. Despite the low diagnostic specificity, this parameter is widely used. The available Guidelines, including the Clinical Laboratory Standards, provide extremely contradictory guidance on the permissible storage period before analysis for this test, namely between 2 and 72 hours. The aim of the study was to investigate the stability of the ESR samples. For that reason, a test on 100 patients was conducted.

METHODS: The investigation was conducted with adult ambulatory patients. Three samples were taken from each subject at the same time point. Those samples were stored under appropriate storage conditions. The samples were stored for a different period of time and then analysed in the same analytical runs using the same validated clinical laboratory method. In order to minimize the chance of errors, at every chosen time point, the three samples were analysed in parallel. The further statistical analyses were performed using the mean value of the three results.

RESULTS: For every patient, several mean values for ESR were obtained - one for every time point. The results were compared with regards to the statistical differences and the clinically significant difference as well. 
CONCLUSIONS: The data processing concluded that there is no statistically significant deviation between the mean values obtained from the ESR measurement of different time points. The implication from the performed investigation is that there are no significant deviations of the results and the overall stability of the samples is approximately 24 hours.

Key Words: ESR, stability

\title{
EVALUATION OF THE INFLUENCE OF THE STORAGE CONDITIONS AND THE TYPE OF THE TUBE IN THE ESR RESULT MEASURED USING DIFFERENT METHODOLOGY
}

\author{
$\underline{\text { R. Mihaylov }}^{2}$, B. Pencheva ${ }^{1}$, D. Stoeva ${ }^{1}$, R. Petkova ${ }^{1}$, N. Marinova ${ }^{1}$ \\ "'SMDL - Ramus" Ltd., Sofia, Bulgaria \\ ${ }^{2} \mathrm{MC}$, ,Y. Filaretova“, Sofia, Bulgaria
}

BACKGROUND-AIM: The ESR test is known with its low diagnostic specificity, but is still widely used. Aim: a comparison of the results for ESR obtained by iSED analyser for automatic analysis from K2-EDTA tubes with the results, obtained by the micro-ESR method (modified Panchenkov micro method) and with BD Seditainer® tubes, containing Sodium Citrate.

METHODS: The investigation was conducted with seventy adult ambulatory patients, whose blood samples were taken with the following test tubes and tested by the described methods: a) Vacuum test tube containing K2-EDTA, 3 ml, iSED automatic analyser, Alcor Scientific, USA. The analyser methodology is photometric rheology used to measure the aggregation of the red blood cells. The result is given after 20 seconds; b) BD Seditainer ${ }^{\circledR}$ test tubes with Sodium Citrate 0.105M, $5 \mathrm{ml}$, BD (Becton Dickinson), USA. The result is provided after 60 seconds, evaluated on a scale of the rack; c) Sodium Citrate 3.8\%, $150 \mu \mathrm{l}$ micro test tubes, and dosing capillary tube, Kabe Labortechnik, Germany, for ESR result provided after 60 minutes, evaluated on a scale of the rack.

RESULTS: All patient samples were analysed using the three methods. The obtained results covered the entire reference range and outside of the reference range, between 5 and $135 \mathrm{~mm} / \mathrm{h}$. The comparison of the results obtained by iSED automatic analyser with the results obtained with the manual method with BD Seditainer ${ }^{\circledR}$ test tubes provided the following results: a) Intercept $\mathrm{A} y=3.3088$ is within the $95 \% \mathrm{CI}$; $\mathrm{b}$ ) Slope $\mathrm{B}=0.8629$ is within the $95 \% \mathrm{CI}$; RSD $=19.1941$ is within the provided interval. The comparison of the results obtained by iSED automatic analyser with the results obtained with micro-ESR method provided the following results: a) Intercept A y=-6.6000 is within the $95 \%$ CI; b) Slope $\mathrm{B}=1.2000$ is within the $95 \% \mathrm{CI}$; $\mathrm{RSD}=14.2267$ is within the provided interval.

CONCLUSIONS: The results observed demonstrate that there are no significant differences between the three investigated methods. The significant advantages of the iSED automatic system - high stability of the samples, minimal volume of the blood sample, the provided option for QC and implementation of LIS, makes this option highly suggested for usage in the routine CL practice

Key Words: ESR, test tubes

\section{THE EFFECT OF HEMOLYSIS AND STORAGE CONDITIONS ON INSULIN STABILITY}

\author{
$\underline{\text { S. Muhtaroglu }}^{1}$, D. Barlak Keti ${ }^{1}$ \\ ${ }^{1}$ Department of Medical Biochemistry, Erciyes University Faculty of Medicine, Kayseri, Turkey
}

BACKGROUND-AIM: Biochemical or spectrophotometric measurements are known to be more affected by hemolysis when compared to immunochemical analysis. This condition can lead to less consideration on immunochemical assays. Threshold values at which haemolysis affects immunochemical tests are indicated in the our kit inserts, but there is no value related to insulin. The aim of this study is to examine the effect of hemolysis and storage conditions on serum insulin stability.

METHODS: Serum pools were formed from the samples reaching the laboratory. The serum samples was transferred to seven tubes in equal volume. In the first tube, only the serum; the second tube, serum + assay diluent and the 3-7 tubes; serum + hemosilate were placed thus samples with haemolysis indexes of 50, 100, 200, 400 and 800 were obtained. In addition, insulin levels were measured in the patient samples with hemolysis index $<20(n=10), 20-50(n=10)$ and 50-100 $(n=10)$ immediately and after 8 hours at room temperature. In our laboratory, immunoassay analysis were done between 8 am and 5 pm. Insulin levels were detected on Cobas e602 (Roche Diagnostics GmbH, Mannheim, Germany). RESULTS: Insulin was found to have 10\% negative bias value below the hemolysis index of $200 \mathrm{mg} / \mathrm{dL}$, if samples were analyzed immediately after centrifugation. Negative bias was found $\leq 10 \%, 27.6 \%$ and $29.5 \%$, in the samples with hemolysis index $<20,20-50$ and $50-100$ respectively that waiting at room temperature for 8 hours.

CONCLUSIONS: Conclusions: Even if there is very little hemolysis (20-50), insulin analysis is not appropriate in waiting samples 8 hours. The hemolysis index should be considered when insulin levels are reported.

Key Words: hemolysis, insulin, stability 


\title{
Demand management
}

\section{ROLE OF ROUTINE ANALYTICAL STUDIES IN THE PREPARATORY OF PEDIATRIC SURGERY. LESS IS MORE}

\author{
M.A. Cuadrado-Cenzual ${ }^{4}$, C. Bailen ${ }^{1}$, A. Garcia ${ }^{1}$, L. Collado Yurrita ${ }^{3}$, C. Soto Beauregard ${ }^{2}$ \\ ${ }^{1}$ Hospital Clínico San Carlos. Analisis Clínicos, Madrid, Spain \\ ${ }^{2}$ Hospital Clínico San Carlos. Pediatrics Surgery, Madrid, Spain \\ ${ }^{3}$ Hospital Clínico San Carlos. UCM, Madrid, Spain \\ ${ }^{4}$ HOSPITAL CLINICO SAN CARLOS. UCM, Madrid, Spain
}

BACKGROUND-AIM: Preoperative Pediatric examinations in most cases, do not respond to the clinical needs. Most of the altered results obtained can be previously known and even predictable from the patient's clinical history and they rarely lead to changes in the therapeutic attitude.The elimination of preoperative tests not indicated, increases the quality of the process by not subjecting the patient to a possible surgery delays. AIM: To determine the value of routine tests in the pediatric surgical preoperative in scheduled surgeries that undergo a general anesthesia procedure.

METHODS: 1st semester of 2016, the requirements of the analytical tests were established in relation to age, surgical risk and type of surgery. For the evaluation of compliance of the consensual procedure, we have reviewed the preoperative analytical studies performed on 846 surgical pediatric patients from June 2016 to January 2018.

RESULTS: 846 surgical operations of scheduled minor surgery were performed. A total of 1185 analytics were carried out. In $8.6 \%$ of the cases, abnormal results were found.19 children presented hemoglobins less than $8.7 \mathrm{mg} / \mathrm{dL}$. 58 children presented platelet numbers below 50,000, which in $97 \%$ of the cases were false decreases. In 92 cases, hyponatremia occurred, which in $78 \%$ of the cases were due to inadequate extraction of the route. All these alterations were not a reason to suspend the surgery. Regarding postsurgical complications: there was no relationship with the preoperative laboratory alterations nor could they have been prevented by results.

CONCLUSIONS: Pediatric patients undergoing surgical procedures of minor surgery, the analytical tests performed routinely in the preoperative period have a very limited value in the management of the patient in pediatric surgery.

Key Words: preanalytical phase

\section{DEMAND MANAGEMENT IN SEROLOGICAL DIAGNOSIS OF RUBEOLA}

L. Arribas Garcia ${ }^{1}$, A. Betrán Escartín ${ }^{1}$, M. Mairal Claver ${ }^{1}$, A. Bellés Bellés ${ }^{2}$, A. Milagro Beamonte ${ }^{3}$, E. Alós Coronas ${ }^{1}$, A. Arcos Galeote ${ }^{1}$, C. Pinilla Mainar ${ }^{1}$, A. Ubieto Roldán ${ }^{1}$, L. Torres Sopena ${ }^{1}$

${ }^{1}$ Microbiology and Parasitology Laboratory, Hospital General San Jorge, Huesca, Spain

${ }^{2}$ Microbiology and Parasitology Service, Hospital Universitari Arnau de Vilanova, Lleida, Spain

${ }^{3}$ Microbiology and Parasitology Service, Hospital Universitario Miguel Servet, Zaragoza, Spain

BACKGROUND-AIM: The detection of IgG against Rubella is a test that is requested for various purposes, mainly for the screening of the first trimester of pregnancy and, in recent years, for the control of patients who are going to be subjected to biological treatments. However, in many cases, this test has already been requested previously and what is done is a duplication of the requests. The objective of this work is to know the economic savings that this non-performance of this test entails in patients who already have a positive result and, therefore, would not require its determination.

METHODS: From January 2016 to December 31 of the same year, a total of 1306 IgG tests against Rubella were requested. All them were reviewed and those with previous positive results were rejected.

RESULTS: Finally, 322 out of a total 1306 Rubella IgG test (24.65\%) were not done because they already had a positive previous result. Those requested from the Obstetrics and Gynecology Service were 611 and 202 (33.87\%) were not performed. In Primary Healt Care a total of 293 requests were made in pregnant women and 78 were rejected (26.62\%). The exact economic cost is difficult to calculate since the times of technicians are not taken into account nor of facultative or other aspects that also could be relevant at the time of knowing the exact amount. If we take into account that the expense of consumables is approximately $4 € /$ determination, $1,288 €$ have been saved, without taking into account the previous mentioned data.

CONCLUSIONS: Health resources are limited, so demand management will be a highly cost-effective action that should be carried out routinely. It is important to reach a consensus with the requesting doctors to avoid unnecessary requests and, in this way, contribute to the decrease in health expenditure.

Key Words: demand management, rubeola, serology 


\title{
DEMAND MANAGEMENT FOR HELICOBACTER PYLORI ANTIGEN DETECTION IN FECAL SAMPLES
}

\author{
$\underline{\text { A. Bellés }}{ }^{1}$, A. Bernet ${ }^{1}$, J. Aramburu ${ }^{1}$, I. París ${ }^{1}$, M. Cruellas ${ }^{1}$, M. García-González ${ }^{1}$, M. Ibarz ${ }^{1}$ \\ ${ }^{1}$ Sección Microbiología, laboratorio Clínico ICS. IRB Lleida, Spain
}

BACKGROUND-AIM: Helicobacter pylori (HP) is responsible for most of gastritis and stomach or small intestinal ulcers. Symptoms may include abdominal pain, nausea or anorexia. HP test is excessively requested in Microbiology labs, even in patients without gastrointestinal symptoms. The aim of this study was to assess the improvement of the fulfillment of the clinical suspicion of patient by requesting doctors, after HP test demand managing implantation.

METHODS: HP antigen detection solicitudes from April to September 2018 were included in the study. Data was obtained from informatics lab system (Modulab, Werfen). The accomplishment of clinical suspicion or diagnosis was analyzed. These solicitudes were revised and separated according clinical information about diagnosis of patient in two groups: "Related diagnosis (RD)" includes those with related information. "Unrelated diagnosis (UD)" includes those with unrelated information. Demand managing for HP test began on July 2018: samples requested for HP test without related diagnosis were rejected and requesting doctors were informed. Statistical analysis (Chi-square test) was performed with the statistical program PSPP to compare results obtained 3 months before (Period 1) and 3 months after (Period 2) implantation of this pre-analytical action. Differences were statistically significant when $\mathrm{p}<0.05$.

RESULTS: We studied 3175 requests. 1880 of them were received in Period 1, and 911 (48.46\%) were included in RD group. In the period 2, 1295 solicitudes were received and $800(61.77 \%)$ were included in RD group. Statistically significant differences $(p<0.05)$ were detected between requests accomplished before and after implantation of demand management for HP test.

CONCLUSIONS: Information related to HP infection increased, therefore this data suggest that this pre-analytical action has improved the fulfillment of the clinical suspicion of patient by requesting doctors.

Key Words: Helicobacter, antigen detection, demand management

\section{DEVELOPMENT AND VALIDATION OF AN IT-BASED ALGORITHM FOR APPROPRIATE TUMOR MARKER RETESTING}

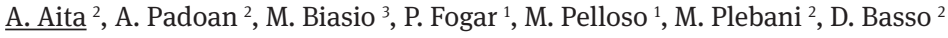 \\ ${ }^{1}$ Department of Laboratory Medicine, University-Hospital, Padova, Italy \\ ${ }^{2}$ Department of Medicine-DIMED, University of Padova, Italy and Department of Laboratory Medicine, University-Hospital, Padova, Italy \\ ${ }^{3}$ Information Technology University-Hospital, Padova, Italy
}

BACKGROUND-AIM: The use of tumor markers (TM) is appropriate mainly for monitoring. The minimal retesting intervals (MRI) vary and depend on TM, tumor type and monitoring time (early/late follow-up). Our aim was to develop and validate an informatic algorithm (IT-TM) supporting appropriate TM retesting.

METHODS: IT-TM was developed for CEA, CA 15-3 and PSA and applied to the University-Hospital of Padova (UH-Pd) IT system for requiring clinicians. Different MRIs were identified (guidelines and sharing with clinicians): 1, 3 and 6 months for post-surgery, early and late follow-up respectively. When MRI is less than 1 year, IT-TM requires the choice (drop-down menu) of the clinical reason for retesting including the possibility to overcome any limitation by selecting "suspect of new tumor". TM requests from external hospitals (Ext-H) were not IT-TM guided. TM requests trend before (2 years) and after (2 years) IT-TM application (October 2016) was evaluated. For comparison AFP, CA 19-9 and CA 125 were included. RESULTS: In UH-Pd, PSA requests declined $(\mid 2: \mathrm{p}<0.001$, ptrend $<0.001)$, while they increased in Ext-H $(\mid 2: \mathrm{p}<0.0001$, ptrend $<0.0001)$. CEA did not vary in UH-Pd nor in Ext-H (p:ns). CA 15-3 was reduced in UH-Pd only $(\mid 2: \mathrm{p}=0.2167$, ptrend $=0.0433)$. AFP, CA 19-9 and CA 125 did not vary over the 4-years study in UH-Pd, while in Ext-H AFP $(\mid 2: \mathrm{p}=0,016$, ptrend $=0.042)$ and CA 19-9 $(\mid 2: \mathrm{p}=0.0028$, ptrend $=0.0003)$ increased. 1425 retesting were registered in IT-TM: 63\% CEA, 19\% PSA and 18\% CA 15-3. Retesting was higher in medical area (67\%), surgery (12\%) and gastroenterology (9\%), and lower in oncology (3\%) and urology (0.4\%). For CEA, 57\% retesting reason was "suspected new tumor".

CONCLUSIONS: IT-TM guided tool for appropriate TM retesting was demonstrated to reduce the overall number of PSA and CA 15-3, not of CEA requests. The success of the IT-TM was mainly observed in specialized areas, its failure in general medical area. Our data indicates that TM retesting, CEA in particular, is often inappropriate.

Key Words: minimum retesting interval, tumor markers, appropriatness

\section{THE MANAGEMENT OF URGENT BIOLOGICAL EXAMINATIONS: EXPERIENCE OF THE BIOCHEMISTRY LABORATORY OF THE MOHAMMED VI UNIVERSITY HOSPITAL OF OUJDA}

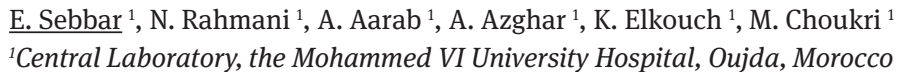


BACKGROUND-AIM: The results of the urgent biological examinations are made within a time that responds to the emergency situation. This delay is by definition the time between the taking of the sample and the communication to the prescriber of the result validated by the medical biologist. The objective of our work is to present the experience of the Mohammed VI University Hospital of Oujda in the management of urgent biological examinations.

METHODS: The Mohammed VI University Hospital of Oujda has a hospital management and information system "Hosix" which covers all areas of activity of the health environment and directly connects and integrates them. The circuit of analysis in medical biology is completely computerized from the medical prescription to the performance of the results. Our laboratory has set up a specific circuit for the urgent biological examinations by the realization of the specific procedures and the mode of management of these examinations, to optimize the duration of realization of the examination and the yield of the results in the short time.

RESULTS: Preanalytical steps prior to receipt of the urgent biological sample in the laboratory are not directly controlled by the laboratory of medical biology. In this case, the mastery of this step must be obtained by an efficient organization, communication and contracting. The laboratory of medical biology specifies in its clinicobiological agreement that the respect of the delays of routing conditions the respect of the global commitments of delay of rendering.

CONCLUSIONS: The standards NF EN ISO 15189 and NF EN ISO / CEI 17025 define the general requirements concerning the quality and the competence of the laboratories of medical biology. The objective of good management of urgent biological examinations is to allow optimal patient management and to better meet the needs of clinicians.

Key Words: management, urgent biological examinations, medical biology

\title{
IMPORTANCE OF EVALUATION OF THE RISK FACTORS OF VITAMIN D DEFICIENCY: STUDY IN THE FRAMEWORK OF THE EVALUATION OF THE VITAMIN D STATUS IN THE POPULATION OF EASTERN MOROCCO
}

\author{
$\underline{\text { E. Sebbar }}^{1}$, N. Rahmani ${ }^{1}$, A. Aarab ${ }^{1}$, A. Azghar ${ }^{1}$, K. Elkouche ${ }^{1}$, M. Choukri ${ }^{1}$ \\ ${ }^{1}$ Central Laboratory, the Mohammed VI University Hospital, Oujda, Morocco
}

BACKGROUND-AIM: Because of the importance of the prevalence of hypovitaminosis D in the general population, the identification of individuals who are at high risk of vitamin D deficiency is a major public health task. The objective of our study is to evaluate pre-analytical factors that influence the status of vitamin D in the population of eastern Morocco.

METHODS: A questionnaire for assessing factors influencing the status of vitamin D was tested on a sample of 1,000 healthy subjects. These factors include age, sex, skin phototype, clothing habits, sun exposure, type of dwelling, and dietary vitamin D intakes.

RESULTS: The study population consisted of 606 women (60.6\%), 394 men (39.4\%), and the average age of participants was $37.33 \pm 11.42$ years. $71 \%$ of the participants had Light brown skin or brown skin, against $29 \%$ dark brown or black skin. About $78 \%$ of the participants had a sun exposure of less than 30 minutes a day. In addition, $66 \%$ of the participants had homes that were not sunny. The average daily vitamin $\mathrm{D}$ consumption of the study population is $3.4 \int \mathrm{g}$ per day, which was well below the recommended daily intake. Females and subjects aged 60 and older are the most affected by this vitamin D malnutrition.

CONCLUSIONS: The results of our study are worrisome; hence the need for awareness and prevention measures that should be conducted in the general population to eliminate the risk factors for hypovitaminosis D.

Key Words: Vitamin D, deficiency, risk factors, public health

\section{INVESTIGATION OF UNNECESSARY LABORATORY TESTING FOR FREE-PSA TEST}

\author{
E. Paydas Hataysal $^{2}$, B. Saraçlıgil ${ }^{1}$, H. Vatansev ${ }^{2}$ \\ ${ }^{1}$ KARATAY UNIVERSITY, FACULTY OF MEDICINE,Department of Biochemistry,Konya,Turkey \\ ${ }^{2}$ SELCUK UNIVERSITY, FACULTY OF MEDICINE,Department of Biochemistry, Konya,Turkey
}

BACKGROUND-AIM: Laboratory tests are important for the confirmation of clinical preliminary diagnoses and appropriate treatment protocols for patients.Free prostate specific antigen (fPSA) consists of circulating PSA unbound to serum proteins. Prostate cancer is the most commonly diagnosed cancer among elderly men. The most common used biomarker to detect prostate cancer in the general population is measurement of circulating PSA. However, PSA values increase also in benign conditions.The percentage of measured circulating PSA existing in free form (free:total PSA ratio) is useful in determination of risk of prostate cancer in patients with borderline or moderately increased total PSA (4.0-10.0 ng/mL) and has been used to help distinguishing prostate cancer from benign prostatic conditions.Our aim was to evaluate the unnecessary laboratory testing for fPSA tests by examining the TPSA and fPSA tests ordered simultaneously.

METHODS: Simultaneously serum total PSA and free PSA test results of patients who admitted to Selçuk University Medical School Hospital between Nov,2016 and Nov,2018 were investigated, retrospectively. Our study includes 2278 Subjects with total PSA measurements and simultaneously free PSA measurements. Total and free PSA levels were measured by Roche Cobas e170 with ECLIA method. 
RESULTS: Overall, $74.5 \%$ of fPSA tests were performed when the total PSA was outside the range of 4 to $10.0 \mathrm{ng} / \mathrm{mL}$, and $59.5 \%$ were performed when the total PSA was lower than $4 \mathrm{ng} / \mathrm{mL}$ and $15 \%$ were greater than $10 \mathrm{ng} / \mathrm{mL}$.

CONCLUSIONS: We found a substantial proportion of free PSA testing occurring outside of the indicated total PSA range. The results of our study on unnecessary test requests indicate the importance of using the guidelines and algorithms during the test request. Unnecessary test requests increase labor and costs. Sharing the information of increase in laboratory costs with clinicians, increasing clinician education programs and applying reflex testing can be effective in reducing unnecessary laboratory testing.

Key Words: free PSA, unnecessary test requesting, preanalytical phase

\title{
INTEREST OF BIG DATA IN THE PREDICTION OF THE URINARY INFECTION: EXPERIENCE OF THE MICROBIOLOGY LABORATORY OF THE MOHAMMED VI UNIVERSITY HOSPITAL OF OUJDA
}

\author{
$\underline{\text { N. Rahmani }}{ }^{2}$, E. Sebbar ${ }^{2}$, C. Lotfi $^{1}$, S. Rifai ${ }^{2}$, S. Lamrabat $^{2}$, M. Bensaleh ${ }^{2}$, A. Maleb ${ }^{2}$ \\ ${ }^{1}$ Computer service, the Mohammed VI University Hospital, Oujda, Morocco \\ ${ }^{2}$ Microbiology laboratory, the Mohammed VI University Hospital, Oujda, Morocco
}

BACKGROUND-AIM: The objective of our study was to explore the utility of the massive data analysis of the Sysmex UF-1000i automaton as a rapid screening tool for urinary tract infections and the predictive ability of the bacterial form (Cocci or Bacillus) to accelerate the return on results, especially in emergencies.

METHODS: A Big Data production cluster has been set up which allows the personalization of the requests for the cytobacteriological examination of the urine (CBEU). This system is based on the processing of large amounts of data from all CBEUs carried out in our laboratory from 01 January 2018 to 30 June 2018. The CBEUs were carried out in accordance with REMIC-2015. Cytological examination of the urine was carried out on the Sysmex UF 1000i automaton which allows the evaluation of the dimensional parameters of the bacteria: the anticipated diffusion of the bacteria (B_FSC) and the diffusion of the fluorescent light (B_FLH).

RESULTS: Our prediction system appears useful for a rapid diagnosis of urinary tract infections. Indeed, the analysis of the UF1000i data is able to provide reliable results on the forms of urinary bacteria (Cocci or Bacillus), which will be available in a few minutes and fully automated, without additional equipment. We note that our system becomes more reliable and more efficient as time goes by, and is being verified and validated.

The proper management of urinary tract infections depends mainly on the precocity of the initiation of antibiotic treatment, in practice the microbiological diagnosis requires a duration of incubation which can happen up to $48 \mathrm{~h}$ or $72 \mathrm{~h}$ for the confirmation of the germ in question and the study of the antibiogram.

CONCLUSIONS: In our study, Big Data can provide results that can be returned to the clinician as initial findings and intervene in therapeutic management, especially in the case of emergencies, before the performance of the final results. Our system requires more time to approve its efficiency and reliability.

Key Words: preanalytical phase

\section{APPLICATION OF A SCREENING ALGORITHM FOR URINE CULTURES FROM THE PRIMARY HEALTH TO IMPROVE THE MICROBIOLOGICAL DIAGNOSIS OF URINARY TRACT INFECTIONS}

\author{
$\underline{\text { P. Salas Gómez-Pablos }}^{2}$, E. Jimenez Morgades ${ }^{1}$, S. Martinez Martinez ${ }^{2}$, A. Blanco Suarez ${ }^{1}$, J. Perez Jové ${ }^{1}$ \\ ${ }^{1}$ Microbiology departament, Catlab laboratory, Barcelona, Spain \\ ${ }^{2}$ Preanalytical Departament, Catlab Laboratory, Barcelona, Spain
}

BACKGROUND-AIM: Until 2016, primary care physicians could request urine cultures with no requisites. For those urines with no culture request, no culture was generated in the laboratory even case of pathological screening results. We designed an algorithm with 3 objectives: Improve the positive predictive value (PPV) of primary health centers urine cultures, avoiding unnecessary antimicrobial therapies and antimicrobial resistances emergence. Achieve greater efficiency in technical workloads by decreasing the number of urine culture processed. Decrease the cost effectiveness of urine culture

METHODS: In order to design an algorithm, we retrospectively analyzed the positive and negative urine cultures together with the screening parameters.We selected the screening parameters in strip test and urinary sediment in order to avoid losing any positive cultures. The final algorithm defines urine culture if at least one of next conditions is true: Test strip: Esterases $\geq++$, positive nitrites, urinary sediment $>10$ leukocytes/field. Exceptions to algorithm are: Pregnant women, programed urological manipulation, recurrent UTI and less 1 year old. In these 4 cases, urine culture is always processed. To compare the total number of urine cultures and the positive, contaminated and negative percentages, we retrospectively analyzed urine cultures results 2 years before and 2 years after the algorithm implementation.

RESULTS: After the implantation of the algorithm a decrease of more than $25 \%$ of the number of urine cultures was observed. Due to this, the percentage of positive urine cultures increased around $10 \%$ and the percentage of negative urine cultures decreased also by $10 \%$. The percentage of contaminated urine cultures maintained stable during the 4 years of the study. 
CONCLUSIONS: After the algorithm implementation, we reached a higher PPV and a more profitable cost effectiveness level. The decrease in more than $25 \%$ of the total number of urine cultures implied a decrease in the technical workload.

Key Words: urine cultures, screening, microbiology

\title{
DEMAND MANAGEMENT OF CREATINE KINASE AND VALIDATION OF KEY PERFORMANCE INDICATORS FOR MONITORING
}

\author{
$\underline{\text { A.M. Rodrigo Valero }}^{1}$, M. Milano Molina ${ }^{1}$, B. Blas López ${ }^{1}$, A. Martín Núñez ${ }^{1}$, A. Magai Barallobre ${ }^{1}$, A. Baldominos Cordón ${ }^{1}$, J. Domínguez \\ López ${ }^{1}$ \\ ${ }^{1}$ Clinical Analysis Department, University Hospital of Guadalajara, Guadalajara, Spain
}

BACKGROUND-AIM: The Spanish Ministry of Health promoted a project with the aim of reducing the use of unnecessary interventions that have not proved efficacy, have little effectiveness and were not cost-effective. One of the recommendations was not to use total Creatine Kinase(CKt) for the diagnosis of myocardial infarction(MI) when more sensitive-specific markers were available. The objective of the study was to evaluate the impact of the demand management plan(DMP) and to validate the use of key performance indicators(KPI) for its monitoring in the Emergency Department, based on the criterion of the non-performance of CKt when requested together with hs-cTnI for the diagnosis of MI.

METHODS: The DMP was implemented in September 2017. Requests for CKt and hs-cTnI between January and June 2017 and 2018 were extracted from the Laboratory Information System. The impact of the plan on practitioners' requests was measured with the Demand Management Indicator, DMI $=\mathrm{n}$ hs-cTnI requests without $\mathrm{CKt}{ }^{\star} 100 / \mathrm{n}$ total hs-cTnI. The KPI used for management were: Management Criterion Application, $\mathrm{MCA}=\mathrm{n}$ tests not performed of CKt ${ }^{\star} 100 / \mathrm{n}$ patients with suspected IM, and Tests Requested by Patient, TRP $=\mathrm{n}$ tests requested CKt ${ }^{\star} 100 /$ total number of patients attended. We used MedCalc ${ }^{\circledR}$ for the comparison of proportions.

RESULTS: The DMI had a notable increase after the establishment of the criterion going from $4.31 \%$ to $89.8 \%$, very close to the ideal $100 \%$. With regard to KPI, we obtained for MCA a value of 74.92\% after the implementation of DMP and for TRP a significant decrease from $85.80 \%$ to $58.64 \%$. All inter-annual differences were statistically significant $(\mathrm{p}=0.000)$.

CONCLUSIONS: The DMP has been satisfactory, as a significant reduction is observed in the number of CKt performed, minimising the use of unnecessary and not cost-effective tests. The use of the proposed KPI is appropriate for the monitoring of the DMP as they reflect the decrease in the request of CKt.

Key Words: myocardial infarction,total CK,high sensitivity cardiac troponin

\section{MANAGEMENT OF CREATINE KINASE TEST REQUESTS IN PATIENTS WITH ACUTE CORONARY SYNDROME}

$\underline{\text { P. Salas Gómez-Pablos }}^{2}$, S. Martinez Martinez ${ }^{2}$, V. Camacho Guillén ${ }^{2}$, J.M. Bauça ${ }^{1}$

${ }^{1}$ Laboratory Medicine, Hospital Universitari Son Espases, Mallorca, Spain

${ }^{2}$ Preanalytical, Catlab Laboratory, Barcelona, Spain

BACKGROUND-AIM: Troponin-T (hsTnT) and I are currently the most useful biochemical markers for suspicion of acute coronary syndrome (ACS). According to both international and Spanish national recommendations, creatine kinase (CK) and CKMB should neither be used for diagnosis, nor for follow-up of myocardial infarction. In our hospital, before 2016, the testing protocol for ACS included troponin T and CK. A working group was created in 2016 to improve laboratory requests and it was decided to exclude CK from the profile, although giving the physician's freedom to request CK additionally. Goals: 1 . To verify the physicians' adherence to the recommendation of not doing CK or CK-MB for diagnosis of ACS. 2. To study the behavior of CK requested in cases in which there was suspicion of ACS and Troponin T is below the upper reference value.

METHODS: The total number of hsTnT and CK requests were extracted for years 2015 to 2018 and compared among years. For results with negative hsTnT and a positive CK, the medical records of the patient were examined for the final diagnosis.

RESULTS: In 2015, 6555 hsTnT determinations were performed, 82\% of them were accompanied by a CK analysis request. In 2016, there were $7708 \mathrm{hsTnT}$ requests, $75 \%$ of which had also a CK request. In 2017, after the implementation of the new profile, 7787 hsTnT were requested, and only $16.8 \%$ had a CK in the same request. Up to November 2018, the percentage of troponins together with a CK request was $15 \%$. From 1314 hsTnT + CK requests in 2017, up to 23 patients showed positive CK result while hsTnT was below the upper limit of reference. None of them was diagnosed with an ACS.

CONCLUSIONS: The professionals have adhered correctly to the recommendation of not requesting CK-MB or CK in the study of myocardial injury, resulting in 78\% fewer determinations. Patients medical records show that for those cases in which both tests are requested, CK does not provide extra information to troponin to rule out ACS.

Key Words: troponin, creatine kinase, coronary syndrome 


\title{
SENSIBLE USE OF CARDIAC MARKERS BY RESTRUCTURING LABORATORY PROFILES
}

\author{
$\underline{\text { M.H. Keppel }}{ }^{2}$, U.C. Hoppe ${ }^{1}$, T. Kolbitsch ${ }^{1}$, S. Auer ${ }^{2}$, T.K. Felder ${ }^{2}$, H. Oberkofler ${ }^{2}$, C. Mrazek ${ }^{2}$, E. Haschke-Becher ${ }^{2}$, J. Cadamuro ${ }^{2}$ \\ ${ }^{1}$ Department of Cardiology, Clinic of Internal Medicine II, Paracelsus Medical University, Salzburg, Austria \\ ${ }^{2}$ University Institute for Medical and Chemical Laboratory Diagnostics, Paracelsus Medical University, Salzburg, Austria
}

BACKGROUND-AIM: Laboratory test panels are increasingly ordered non-specifically which leads to reduced pretest probability and increased numbers of false-positives followed by increased inter-disciplinary consultations, costs and increased adverse risk for patients. In collaboration with our cardiology department, we aimed to reduce non-specific ordering of cardiac markers N-terminal pro-brain natriuretic peptide (NTpBNP), and high-sensitive cardiac troponin T (hsTNT).

METHODS: Exclusion of NTpBNP and hsTNT from panels was implemented in several steps at different wards at the Department of Cardiology of the University Hospital Salzburg, a tertiary care center. In June 2017, the staff of a single ward was reminded to actively order NTpBNP only according to current guidelines. From January to February 2018, NTpBNP and hsTNT were removed from panels for all three wards. Endpoints of the current study were absolute number of orderings, length of stay, 30-day re-admission rates of patients with primary and secondary diagnoses of ICD-10 codes I50.x (heart failure) for NTpBNP and codes I20.x-I25.x (ischemic heart diseases) for hsTNT. Data were obtained from local LIS, all analyses were performed in R.

RESULTS: Between January 2017 and September 2018, for all three wards we found a mean reduction of test per month of -300 (-75.8\%) for NTpBNP and of -267 (-64.9\%) for hsTNT. No significant increase in lengths of stay or 30-day re-admission rates could be observed. For NTpBNP, the reduction of multiple tests was more pronounced than for single tests for diagnose codes I50.x. For hsTNT, the proportion of tests for diagnose codes I20.x-I25.x increased.

CONCLUSIONS: In this study, we found great potential for reducing overutilization of cardiac markers by removing them from laboratory panels while not observing adverse increases in patients' length of stay or 30-day re-admission rates.

Key Words: NT-proBNP, high-sensitive cardiac troponin T, laboratori test panel, overutilization, demand management

\section{DEMAND MANAGEMENT ON GENETIC TESTS THROUGH ELECTRONIC ORDERING SYSTEMS: "PREVIOUS RESULTS, ONGOING TESTS, FUTURE REQUESTS"}

\author{
$\underline{\text { A. Corchón-Peyrallo }}^{1}$, M. Díaz-Giménez ${ }^{1}$, A. Pozo-Giráldez ${ }^{1}$, E. Rodríguez-Borja ${ }^{1}$, A. Carratalá-Calvo ${ }^{1}$ \\ ${ }^{1}$ Hospital Clínico Universitario de Valencia, Spain
}

BACKGROUND-AIM: Most of the Laboratory Information Systems (LIMS) provide internal support rules that could cancel genetic tests performance if there is a previous (and invariable) result. Unfortunately, only some evolved systems allow this cancellation if there is a pending result (due to a long turnaround time) or even if there is a previous order, but for the future test. These softwares prevent unnecessary requests at source prior to phlebotomy, avoiding inconvenience for patients and help to manage test adequacy. We have implemented a new feature in our Electronic Request System (ERS) that checks all these possibilities in order to cancel some genetic tests.

METHODS: Prospective study performed between October 2017 - October 2018. A Clinical decision support rule (CDSR) was implemented for the following genetic tests: HLA - B27, HLA - DQ, Factor V Leiden, Prothrombin G20210, HFE, HLA - B5701, HLA - B51 y Lactose gene. CDSR would cancel test ordering (and it would explain the cause to the provider) if a previous result existed; an ongoing test existed; a future request with the test existed in our request "mailbox". Cancellation implied an internal alert mark for that request with statistical purposes. Total number of cancelled tests, their cause, $\%$ from the total activity and cost savings were calculated.

RESULTS: From 2386 genetic tests ordered, 205 were finally cancelled (8,6\%). From these 205, 151 (74\%) due to a previous result, 23 (11\%) due to an ongoing test and $31(15 \%)$ due to a future request in our "mailbox". The cancelled test represented a cost savings of $12164,6 €$. The most rejected tests were B27 (59), Factor V (57) and G20210 (56).

CONCLUSIONS: We strongly recommend that CDSRs for genetic tests take into account not only previous results in their algorithms but ongoing tests and future requests that include a particular test, given their relevance (26\%) and cost impact.

Key Words: preanalytical phase

\section{“SEND \& HOLD” CLINICAL DECISION SUPPORT RULES (CDSR) FOR TSH IN INPATIENTS AND 1,25 - VITAMIN D: “1. ASK OR SUGGEST, 2. HOLD AND REVIEW Y 3. DECIDE”}

\author{
M. Díaz-Giménez ${ }^{1}$, A. Pozo-Giráldez ${ }^{1}$, A. Corchón-Peyrallo ${ }^{1}$, E. Rodríguez-Borja ${ }^{1}$, A. Carratalá-Calvo ${ }^{1}$ \\ ${ }^{1}$ Hospital Clínico Universitario de Valencia, Spain
}

BACKGROUND-AIM: In electronic requesting systems (ERS) a "send-and-hold" funcionality allows providers to make their tests orders by answering some questions (external restriction), but puts on hold those tests until laboratory professionals review the reasons given and 
decide if they are appropriate or not (internal restriction). Our main aim was to implement two "send-and-hold" rules in order to manage the requesting of inpatiens TSH (where its usefulness is limited) and 1,25-Vitamin D (frequently mistaken with 25-Vitamin D) and evaluate their impact.

METHODS: Prospective study April 2017 - 2018. First step TSH: If a TSH in an inpatient is ordered, our ERS will cancel the test offering a brief explanation unless provider overrides it and specifies an indication in a pop-up window. Endocrinology and Pediatrics services are excluded from this rule. First sept 1,25VitD: If a 1,25 VitD is ordered, our ERS will change it for 25VitD offering a brief explanation unless provider overrides it and justifies its decision in a pop-up window. Nephrology is excluded from this rule. If rules are not overridden, an internal tracer test is created for statistical purposes. Second step (LIMS): The tests are put on hold by our LIMS until review by lab professionals who read the explanations given by providers and decide if the test have to be performed or not.

RESULTS: 1034 TSH and 282 1,25VitD were ordered using our ERS. Rules were not overriden for 561 (54,3\%) and 241 (85,5\%) tests respectively. From the rest of the tests, Lab professionals also cancelled 164 TSH and 24 1,25VitD due to unjustified or inappropriate reasons. Only 309 TSH and 17 1,25VitD were finally performed. "Send-and-hold" system finally rejected 70,2\% and 94\% from the total TSH and 1,25 VitD ordered respectively.

CONCLUSIONS: "Send \& hold" CDSRs (or two steps rules) show an extraordinary efficacy as a test management strategy allowing a proper management of clinical exceptions without loss in their automatism.

Key Words: demand management, hormones test, cost savings, “Send \& Hold” IT Systems, laboratory medicine

\title{
NUMBER OF FOLATE AND VITAMIN B12 TEST REQUESTS IN A 6 MONTH PERIOD IN TWO LARGE CAPACITY HOSPITALS OF ISTANBUL: DOES IT SHOW INDISCRIMINATE TEST REQUESTING?
}

\author{
$\underline{\text { E. Serin }}^{2}$, C. Kazezoglu ${ }^{1}$, S. Cigerli ${ }^{2}$, N. Dolu ${ }^{2}$, K. Dogan ${ }^{2}$ \\ ${ }^{1}$ Medical Biochemistry Lab, Kanuni Sultan Suleyman Training\&Research Hospital, Istanbul, Turkey \\ ${ }^{2}$ Medical Biochemistry Lab, Sisli Etfal Training\&Research Hospital, Istanbul, Turkey
}

BACKGROUND-AIM: Drugs used by the patients are not questioned most of the time before blood draw in the pre-analytical phase. Especially vitamin complexes are used routinely by the elderly patients and vitamin B12 (VB12) and folate tests are nearly routinely requested from the same group of patients. Our aim was to analyze the results of requests of these two vitamins to evaluate inappropriate and indiscriminate test requesting.

METHODS: We examined the data of the last six months on two hospitals' laboratory information systems to find the total number of analyzed VB12 and folate tests. Then we searched for the number of tests that were lower than, in between and higher than the reference ranges of the tests. We also searched for the ones that were over the detection limits of our analyzers; i.e. the total number of results yielded as "higher than".

RESULTS: Total number of VB12 and folate tests in Kanuni and Sisli Hospitals were 79096, 44137 and 82668, 56390, respectively. Total number of folate tests lower than, in between and higher than reference ranges in Sisli were 475, 55635 and 2680, respectively. The results for the same parameter in Kanuni were 6276, 36013 and 872, respectively. Total number of VB12 tests lower than, in between and higher than reference ranges in Sisli were 5419, 76415 and 7171; and 4962, 70643 and 5506 in Kanuni, respectively. The number of VB12 and folate tests over detection limits in Kanuni and Sisli were 491, 1848 and 834, 280 for VB12 and folate, respectively. Number of re-requested folate tests in 15 days in Sisli and Kanuni were 2 and 35, and the number of re-requested VB12 tests in Sisli and Kanuni were 25 and 20, respectively.

CONCLUSIONS: Besides the extra costs paid for the analyzed tests which gave no results, the time spent, the tubes wasted and most importantly the patients' blood drawn to no purpose should also be of concern in healthcare management.

Key Words: drug utilization, pre-analytical error, test re-request

\section{NT-PROBNP DEMAND MANAGEMENT EVOLUTION}

\author{
$\underline{\text { A. Rakovac Tisdall }}^{1}$, A. Leonard ${ }^{1}$, R. Srinivasan ${ }^{1}$, G. Boran ${ }^{1}$ \\ ${ }^{1}$ Clinical Chemistry, Laboratory Medicine Department, Tallaght University Hospital, Dublin 24, Ireland
}

BACKGROUND-AIM: The plasma concentration of N-terminal pro-B type natriuretic peptide (NT-proBNP) is used as a primary diagnostic test in heart failure, not recommended for treatment effect monitoring. Due to significant assay costs, we introduced a demand management process in 2013, capping the number of analyses to 2 per patient/admission, at diagnosis and pre-discharge. Following substantial cost increases, we audited the requesting process in 2018.

METHODS: All NT-proBNP requests in September 2018 were extracted through the laboratory information system and reviewed by the laboratory medical team. The number of repeat analyses, time interval between repeats, the day of the week of sample request, sample origin and clinical subspecialty of the requesting team were analysed. 
RESULTS: From the total of 581 samples on 478 patients, 180 samples on 77 patients were repeats. Three-quarters of patients with repeat analyses had 1 repeat, 14 patients had 2 repeats, 2 patients three, and 2 patients four repeats. Nearly half of all repeats (48\%) were requested within 72 hours of the initial sample, $90 \%$ of them on inpatients. We expected this deviation from the protocol to be explained by samples arriving on patients admitted over the weekend as NT-proBNP is not processed outside regular working hours. Surprisingly, most repeat requests came in Monday-Friday (89\%). Furthermore, as it is clinically unlikely for a patient with acute heart failure to be fit for discharge within 72 hours, it is most probable that nearly half of all repeated samples were clinically questionable. This amounts to an unnecessary spend of $€ 750$ in one month on reagents only, approximating €9000 a year.

CONCLUSIONS: A more stringent protocol with additional checkpoints in the total testing process has been implemented as a result of this audit. Taking into account a $6.8 \%$ increase in testing workload a year, we expect a saving of at least €10000 in 2019, while providing a clinically streamlined heart failure diagnosis support service.

Key Words: NT-proBNP, demand management, heart failure biomarker

\title{
Technology solutions
}

\section{THE PATIENT IDENTIFICATION ERRORS IMPACT IN THE HOSPITAL AND THE ORGANIZATION}

\author{
M.A. Cuadrado-Cenzual ${ }^{3}$, C. Bailen ${ }^{1}$, M.J. Ciudad ${ }^{4}$, A. Garcia ${ }^{2}$, C. Luis ${ }^{4}$ \\ ${ }^{1}$ Hospital Clínico San Carlos. Analisis Clínicos, Madrid, Spain \\ ${ }^{2}$ Hospital Clínico San Carlos. Analisis Clinicos, Madrid, Spain \\ ${ }^{3}$ Hospital Clínico San Carlos. Analisis Clinicos, Madrid, Spain \\ ${ }^{4}$ Hospital Clínico San Carlos. UCM, Madrid, Spain
}

BACKGROUND-AIM: Patient Safety has emerged as a key dimension of quality in hospital worldwide. Failures in Identification Patient causes patient adverse events and a economical consequences in the hospital organization. The objectives of this study were analyze patient identification errors (PIE) in analytical requests and determine patient consequences and the costs associated with such adverse events.

METHODS: Analysis of PIE occurred on a total of 414.052 analytical requests performed in emergency and hospitalized patients. The results were statistically analyzed using the chi-square test and the SPSS v15.0. The costs analysis were carried out based on redundant tests, repeated sampling, time spent by health workers and the impact on average patient stay.

RESULTS: A total of 688 PIE were detected. 11.854 additional tests were requests, and 681 redundant blood sampling extractions were realized. The costs in this area was 14.420 euros. Regarding the Staff costs: the time spend by Professional Staff (Doctor, Nurse and laboratory staff) represent 21.347 Euros. Finally, in relation with the impact on patient stay at the hospital, it was observed that PIE caused a delay in the reporting of results. (Average 3.8 hours, range 1.2-5.3 hours) in emergency and hospitalized patients. In 63\% hospitalized patients, this delay caused an increase of average stay. The delay costs was 78.987 Euros.

CONCLUSIONS: Economical impact of PIE associated with analytical requests are specially relevant in order to implement strategies to reduce errors and to improve health risk management.

Key Words: patient security, patient identification, accessibility, pediatric surgery

\section{CELL FREE DNA ISOLATION FROM PERIPHERAL BLOOD: IMPACT OF PRE-ANALYTICAL VARIABLES}

D. Bozzato ${ }^{3}$, C.F. Zambon ${ }^{1}$, A. Padoan ${ }^{3}$, M. Montagnana ${ }^{4}$, M. Benati ${ }^{4}$, E. Danese ${ }^{4}$, A. Aita ${ }^{3}$, S. Moz ${ }^{3}$, F. Navaglia ${ }^{2}$, V. Aneloni ${ }^{5}$, M.G. Epifani 2, D. Basso ${ }^{3}$, G. Lippi ${ }^{4}$, M. Plebani ${ }^{3}$

${ }^{1}$ Department of Biomedical Sciences and Department of Laboratory Medicine, University of Padova, Italy

${ }^{2}$ Department of Laboratory Medicine, University of Padova, Italy

${ }^{3}$ Department of Medicine and Department of Laboratory Medicine, University of Padova, Italy

${ }^{4}$ Section of Clinical Biochemistry, University of Verona, Verona, Italy

${ }^{5}$ Transfusion Medicine and Immune-Hematology, University of Padova, Italy

BACKGROUND-AIM: Pre-analytical phase standardization is a key step in translation of ccfDNA analyses to clinic. We aimed to verify the effect on ccfDNA isolation of collection tube, processing time and method of extraction.

METHODS: Blood from 10 donors was collected in $\mathrm{K}_{2}$ EDTA tubes (BD) and PAXgene Blood ccfDNA tubes (Preanalytix), kept refrigerated (4 ${ }^{\circ} \mathrm{C}$ ), centrifuged once after $2 \mathrm{~h}$ or $72 \mathrm{~h}$. ccfDNA extraction from plasma was performed by four commercial kits: Quick-cfDNA Serum \& Plasma 
Kit (Zymo Research) (A), Maxwell RSC ccfDNA Plasma Kit (Promega) (B), QIAamp MinElute ccfDNA Midi Kit (QIAGEN) (C), Helix Circulating Nucleic Acid (Diatech Pharmacogenetics) (D). Starting plasma volume (4mL) and ccfDNA elution volume (80 (L) were standardized for all kits. Extracted DNA quantity was determined by fluorimetric assay (Qubit ${ }^{\circledR}$ dsDNA HS Assay Kit, Thermofisher). Leukocyte gDNA contamination was evaluated as DNA integrity index by RealTime Alu PCR. ccfDNA recovery was measured by RealTime PCR, targeting exogenous DNA spiked in blood samples (TATAA Universal DNA Spike 166 bp kit, TATAA Biocenter AB).

RESULTS: Extracted DNA concentration was $0.40 \pm 0.45 \mathrm{ng} /$ ( L (mean \pm SD) being significantly higher only in K2EDTA tubes processed at $72 \mathrm{~h}\left(0.94 \pm 0.58 \mathrm{ng} / \int \mathrm{L}\right)(\mathrm{p}<0.001)$. DNA integrity index was $0.43 \pm 0.26$ (mean $\pm \mathrm{SD}$ ) being gDNA contamination significantly increased only in K $\mathrm{E}_{2}$ DTA tubes processed at $72 \mathrm{~h}(0.83 \pm 0.18)(\mathrm{p}<0.001)$. Mean ccfDNA recovery was $56.43 \pm 31.55 \%$ being significantly associated with the extraction kit $(A=38.14 \pm 26.80 \% ; B=67.92 \pm 31.27 \% ; C=67.85 \pm 31.98 \%$ and $D=48.60 \pm 25.96 \%)(p<0.001)$, independently from type of blood collection tube $(\mathrm{p}=0.151)$ and processing time $(\mathrm{p}=0.468)$.

CONCLUSIONS: ccfDNA signals are stable ex vivo up to $72 \mathrm{~h}$ before processing, independently from type of blood collection tube. ccfDNA contamination by leukocyte gDNA can be avoided by dedicated collection tubes. The protocol adopted for ccfDNA extraction influences its recovery.

Key Words: ccfDNA, collection tube, processing time, method of extraction

\title{
POINT-OF-CARE MEASUREMENT OF BLOOD BETA-HYDROXYBUTYRATE OF CHILDREN WITH DRUG-RESISTANT EPILEPSY
}

\author{
A. Ivanov ${ }^{1}, \mathrm{U}$. Vaher $^{2}$ \\ ${ }^{1}$ Clinical Chemistry and Laboratory Hematology Department, United Laboratories, Tartu University Hospital, Estonia \\ ${ }^{2}$ Department of General Pediatrics and Neurology, Children’s Clinic, Tartu University Hospital, Estonia
}

BACKGROUND-AIM: Incidence of childhood epilepsy in Estonia is 80:100 000. Up to 70\% of patients have their seizures controlled with antiepileptic drugs. For some children who continue to have seizures the ketogenic diet (KD) may help to reduce the number of severity of seizures. The KD is a special high-fat, low-carbohydrate diet that helps to control seizures. It is prescribed by a physician and carefully monitored by a $\mathrm{KD}$ team. Guidelines advocate measurement of ketones for the management of KD. Aim: To compare ketone measurement in urine and blood and estimate the effect to the management of KD.

METHODS: Case histories were retrospectively reviewed all of the patients who had KD. Epilepsy course, etiology and outcome and laboratory data were collected and analyzed. Four children with drug-resistant epilepsy were introduced the KD, in two of them ketones were measured from urine (group1, before POCT BHB) and in two of them from blood (group2). Ketones measurement was performed regularly 1- 2 times per day.

RESULTS: Mean glucose concentration, PH and BE of the patients group 1 were significant lower then group 2: 2,76; 7,34 and -8,51 vs 3,35; 7,38 and $-5,71$, respectively. We compared the mean, minimum and maximum values of glucose, $\mathrm{PH}$ and $\mathrm{BE}$ with reference ranges. $90 \%$ of values from group 1 were outside from the reference ranges (except max value of PH 7,39: reference range is 7,35-7,45). From group 2 only 40\% of values (mean and min of $\mathrm{BE}$ and min of glucose and $\mathrm{PH}$ ) were outside from reference ranges.

CONCLUSIONS: Measuring ketones from blood gives more reliable data about the patient's condition and level of the ketosis, which allows take precise actions to manage the diet.

Key Words: ketones, ketogenic diet, glucose

\section{EVALUATION OF CAP-PIERCING TECHNOLOGY FOR COAGULATION TESTING ON SYSMEX® CS-2500 SYSTEM}

$\underline{\text { S. Komljenović }}^{1}$, R. Zrinski Topić ${ }^{1}$, J. Leniček-Krleža ${ }^{1}$

${ }^{1}$ Children's Hospital Zagreb, Institute for laboratory diagnostics, Department of medical biochemistry and hematology, Zagreb, Croatia

BACKGROUND-AIM: Cap-piercing technology is a mechanism that has emerged in order to improve the laboratory staff safety by reducing exposure to the biological specimen. The aim of the study was to examine whether cap-piercing technology is reliable for routine use since nonreproducible results were previously noticed for coagulation tests, noticeably fibrinogen concentration that was measured by Sysmex ${ }^{\circledR}$ CS-2500 System coagulation analyzer.

METHODS: Our study collectively used 58 blood samples from pediatric population standardized for coagulation testing which had their Prothrombin time (PT) measured using coagulometric assay (Dade Innovin, Siemens), fibrinogen concentration measured using coagulometric assay (Dade Thrombin, Siemens), D-dimer concentration measured using immunoturbidimetric assay (Innovance D-dimer, Siemens) and/ 
or Protein C activity measured using spectrophotometric assay (Berichrom Protein C, Siemens) from the capped tube and then the decapped tube. Bland-Altman analysis and Passing-Bablok regression analysis have been used to compare results.

RESULTS: No significant difference has been observed for any of the previously mentioned tests when Bland-Altman analysis (with Westgard's QC rules) has been used: PT had average BIAS $=0.7 \%$ (desirable BIAS $=2.0 \%$ ), fibrinogen had average BIAS $=0.4 \%$ (desirable BIAS $=4.8 \%$ ), Protein $\mathrm{C}$ had average BIAS $=0.2 \%$ (desirable BIAS $=13.9 \%)$ and D-dimer had average BIAS $=0.2 \%($ desirable BIAS $=8.82 \%)$. Passing-Bablok regression analysis showed no significant difference for PT $(\mathrm{y}=[-0.0305-0.0000]+[1.0000-1.0411] \mathrm{x})$, Protein C $(\mathrm{y}=[-2.2598-1.1058]+$ $[0.9843-1.0217] \mathrm{x})$ and $\mathrm{D}$-dimer $(\mathrm{y}=[-0.0249-0.0175]+[0.9688-1.0235] \mathrm{x})$. Passing-Bablok regression analysis for fibrinogen showed systematic and proportional differences $(\mathrm{y}=[0.0087-0.0997]+[0.9547-0.9935] \mathrm{x})$.

CONCLUSIONS: We have concluded that cap-piercing technology is acceptable for routine coagulation testing on Sysmex ${ }^{\circledR}$ CS-2500 System.

Key Words: cap-piercing technology, coagulation, method comparison

\title{
THE USAGE OF POINT-OF-CARE GLUCOSE SCREENING TESTS BEFORE ORAL GLUCOSE TOLERANCE TEST IN MEDICAL OFFICES OF CENTRALIZED LABORATORY
}

\author{
A. Salikhova ${ }^{1}$, I. Torshina ${ }^{1}$, E. Khasyanova ${ }^{1}$, N. Igonina ${ }^{1}$, E. Chashchikhina ${ }^{1}$, E. Kondrasheva ${ }^{1}$, E. Zhuravleva ${ }^{1}$ \\ ${ }^{1}$ LLC Independent Laboratory INVITRO, Moscow, Russia
}

BACKGROUND-AIM: As a centralized laboratory, we use a preliminary glucometer test to solve the safety issue in our own distant offices when individual patients order an oral glucose tolerance test (OGTT). The aim of the study was a retrospective analysis of parallel glucose level measurement using a glucometer for fasting capillary blood glucose (FCBG) testing before OGTT and a biochemical analyzer for the plasma glucose (PG) testing at point 0 (fasting) for the OGTT in non-pregnant and pregnant females. In parallel, we demonstrate applicability of "Quality control-Results management system" program (RMS) to control the quality of glucometer results in the medical offices of a centralized laboratory. METHODS: FCBG were checked on FreeStyle Optium (Abbott) glucometer, PG levels were measured by hexokinase method using Architect c8000, c16000 (Abbott) analyzers. Total sample size was 1800 ( $n=559$ pregnant and $n=1250$ non-pregnant). The significance of the differences between samples was assessed by Mann-Whitney U test (Medcalc).

RESULTS: Correlation analysis showed statistically significant differences between two methods in both groups: $\mathrm{y}=1.06 \mathrm{x}+0.088$ (nonpregnant, $\mathrm{p}<0.0001$ ), $\mathrm{y}=1.37 \mathrm{x}-2.30$ (pregnant, $\mathrm{p}<0.0001$ ). Average biases, percentage in both groups were out of the acceptable ranges of Westgard acceptable bias, but less than $15 \%$. These are acceptable limits for difference between the measurements by glucometer and in the laboratory according to ISO 15197:2013. The results of patient samples monitoring in the RMS demonstrated the absence of abnormally high results in both capillary and venous blood.

CONCLUSIONS: The usage of preliminary point-of-care FCBG allows make a decision regarding the safety of glucose tolerance test. The RMS program helps to monitor glucometer performance in the medical offices network of centralized laboratory.

Key Words: capillary blood glucose, plasma glucose, oral glucose tolerance test (OGTT), POC-screening analysis

\section{COMBINATION OF A NEW INFORMATIC TOOL FOR BLOOD SAMPLE TRACEABILITY AND NURSE EDUCATION IN A FRENCH HOSPITAL}

\author{
$\mathrm{L}$. Tonini $^{2}$, C. Chirica ${ }^{2}$, S. Kowalski ${ }^{1}$, A. Michoud ${ }^{3}$ \\ ${ }^{1}$ Nursing Care Management Unit \& Clinico-biological commission, Grenoble-Alpes Universitary Hospital, Grenoble, France \\ ${ }^{2}$ Pre-analytical Unit of the laboratory, Biological and Pathological Institute, Grenoble-Alpes Universitary Hospital, Grenoble, France \\ ${ }^{3}$ Quality insurance referent of the laboratory, Grenoble-Alpes Universitary Hospital, Grenoble, France
}

BACKGROUND-AIM: Informatic system for test ordering to perform venous blood sample allow traceability. Even though our informatic system provide the option, the validation of the action of blood sampling by phlebotomists was poorly reported. At our initial laboratory accreditation visit in 2014 , only $35 \%$ of samples were validated by nurses, leading to inacurate sampling hours in $75 \%$ of our test ordering. We therefore decided to 1 / improve informatic tool for traceability of blood sampling and 2/ educate phlebotimists.

METHODS: Members of the clinico-biological group: medical staff in charge of pre-analytical phase of the laboratory and nurses from different medical units (intensive care, emergency, pediatric, in house patients units...) defined evolutions of the blood sample tracking informatic module.After 2 months of validation in pilot units, the whole units were equiped progressively, representing 440 bar code scanners installed between september 2015 and december 2016. Each medical units benefit of educationnal of the nurse staff during installation.

RESULTS: Follow up of blood samples traceability was continuously performed and data reported to the clinico-biological group, nursing care and medical management units. For each of the 12 biggest test ordering units, an annual restitution is performed. Since 2014, blood samples traceability constantly improves: 65,2 \% in 2015, 91,5 \% in 2016, and remains above 80\%: 81,2 \% in 2017 and 85,2 \% in 2018. During 
audit period, in 2015, $58 \%$ of blood samples were traced compared to only $35 \%$ of the whole samples during the same period. In $2017,85 \%$ of the blood samples are traced by nurses when audit performed, compared to $82 \%$ for the whole samples of the same period.

CONCLUSIONS: The informatic tool combined to phlebotomists education lead to improvement of blood sample tracking in an audit independent way. This allows to obtain in more than $80 \%$ of blood samples the traceability of phlebotomy steps.

Key Words: traceability, blood sample, time of day, nurse education

\title{
CAPILLARY BLOOD SAMPLING FOR POCT ANALYSIS: LEFT VS. RIGHT HAND
}

\author{
M. Bozovic ${ }^{1}$, L. Milevoj Kopcinovic ${ }^{1}$, J. Culej ${ }^{1}$, A. Vrtaric $^{1}$, A. Topic ${ }^{1}$, M. Miler ${ }^{1}$, N. Nikolac Gabaj ${ }^{1}$ \\ ${ }^{1}$ Department of Clinical Chemistry, Sestre milosrdnice University Hospital Centre, Zagreb, Croatia
}

BACKGROUND-AIM: Point-of-care testing (POCT) comprises diagnostic testing usually performed by non-laboratory personnel at the site of the patient (i.e. hospital wards or at home). The comparability of results obtained from left hand (L) vs. right hand (R) capillary sampling samples using POCT is implied. Our aim was to test this hypothesis, i.e. to test if results obtained from $\mathrm{L}$ and $\mathrm{R}$ hand capillary samples by using a POCT device were comparable.

METHODS: Paired capillary samples were taken simultaneously from L and R middle fingers of 21 healthy participants using Greiner bio-one miniCollect safety lancets with puncture depth of $2.00 \mathrm{~mm}$. A volume of $200 \mu \mathrm{L}$ of capillary blood from each finger was collected using lithium-heparinized Minivette POCT (Sarstedt, Nümbrecht, Germany). Testing was performed using Abbott i-STAT Alinity (Abbott, Abbott Park, USA) POCT analyser and $\mathrm{Na}, \mathrm{K}, \mathrm{Cl}$, iCa, glucose, urea, creatinine, haematocrit, haemoglobin, pH, PCO2, PO2, TCO2, HCO3-, base excess (BE), sO2, lactate were measured. Statistical analysis was performed using MedCalc statistical software (Ostend, Belgium). Mean biases obtained from L vs. R were compared to CROQALM criteria for respective parameters.

RESULTS: No statistical or clinical difference in results obtained after sampling from L and R was found. Calculated biases for each parameter analysed were lower than the criteria previously defined. The biases expressed as mean bias (corresponding criteria) were as follows: $\mathrm{pH}$ 0.04\% (1\%), pCO2 - 0.24\% (8\%), pO2 2.00\% (8\%), lactate -4.35\% (16\%), Na 0.30\% (3\%), K 1.65\% (6\%), Cl - 0.15\% (4\%), urea 0.88\% (8\%), creatinine $1.19 \%$ (9\%), glucose - 0.87\% (7\%), iCa 1.79\% (4\%), Hct 1.25\% (5\%), Hb 1.22 (5\%).

CONCLUSIONS: Results obtained from L and R hand capillary samples using a POCT device were comparable. Thus, capillary sampling might be performed from either hand, interchangeably.

Key Words: capillary blood sampling, POCT, left hand, right hand

\section{INADEQUATE SAMPLE QUALITY AS PREANALYTICAL ERROR INFLUENCES THE INTERPRETATION OF CLINICAL FLOW CYTOMETRY RESULTS}

\author{
$\underline{\text { Z. Hevessy }}{ }^{1}$, M. Száraz-Széles ${ }^{1}$, B. Kárai ${ }^{1}$, G. Ivády ${ }^{1}$, S. Baráth ${ }^{1}$, E. Szánthó ${ }^{1}$, J. Kappelmayer ${ }^{1}$ \\ ${ }^{1}$ Department of Laboratory Medicine, Faculty of Medicine, University of Debrecen, Debrecen, Hungary
}

BACKGROUND-AIM: Several recommendations in the standardization and harmonization of the analytical phase of clinical flow cytometry procedures has been published in the last decade. Recently automatic gating and data analysis has been introduced, which will help to minimize postanalytical errors - the interpretation of data. However, preanalytical errors in the flow labs are neither well documented nor fully elucidated. B-cell precursor acute lymphoblastic leukemia (BCP-ALL) is the most common malignancy of childhood. Day15 bone marrow (BM) testing for minimal residual disease (MRD) by flow cytometry is mandatory for risk stratification and therapy planning. However, bone marrow aplasia and the presence of cell debris makes this specimen difficult to interpret. We aimed to explore the ratio of inadequate samples as sources of preanalytical error and evaluated, how it influenced result interpretation. Another valuable sample, cerebrospinal fluid (CSF) quality was also assessed with or without fixation.

METHODS: Day 15 BM samples of 113 children (97 B-ALL, 22 T-ALL) between 2011 and 2018 and 22 CSF samples without fixative compared to 30 CSF samples with fixative were examined.

RESULTS: Peripheral blood contamination (erythroblast <2\%) was found in 12 out of 91 (13\%) B-ALL and 2 out of 22 (10\%) T-ALL day15 BM samples, thus day 15 MRD could not be reported according to the ALL-IC 2009 flow cytometry protocol. Without fixative 10/22 (45\%) CSF displayed viable cells $<30 \%$ and results could not be reported, while CSF samples with fixative were more adequate and the results could not be interpreted only in 5/30 (17\%) cases.

CONCLUSIONS: MRD results of day15 BM samples in childhood BCP-ALL cases could not be reported in 10.6\% as a result of preanalytical errors, so they could not be used in the prognostic stratification and therapy planning. In case of another unique sample type the application of a special tube with fixative considerably decreased the ratio of inadequate CSF samples.

Key Words: flow cytometry, preanalytical error, sample quality, bone marrow, cerebrospinal fluid 


\title{
POINT-OF-CARE DETECTION OF HEMOLYSIS IN EMERGENCY CARE
}

\author{
$\underline{\text { H. Duhalde }}^{2}$, B. Eriksson ${ }^{2}$, A. Brolinson ${ }^{2}$, L. Hansson ${ }^{1}$ \\ ${ }^{1}$ Department of clinical chemistry, Central Hospital Karlstad, Sweden. \\ ${ }^{2}$ Hemcheck, Karlstad, Sweden
}

BACKGROUND-AIM: The preanalytical phase is accountable for a vast majority of laboratory test errors. Among preanalytical errors, hemolysis is the most frequent error of sample rejection. A new method, Helge (Hemcheck, Karlstad, Sweden), was evaluated at an emergency department in Sweden.

METHODS: 1671 patients (control group $n=774$, intervention group $n=897$ ) attending the emergency department were included and randomized during a period of six months. Helge was utilized in the intervention group. Hemolyzed blood samples ( $>0,5 \mathrm{~g} / \mathrm{l})$ (VACUETTE® Tube $3 \mathrm{ml}$. LH) according to the new method were discarded until new, negative, samples was collected and sent for analysis at central laboratory. Information associated with the blood sampling procedure was collected by nurses in both groups. Hemolysis index collected from Vitros 5.1 (Ortho Diagnostics Inc.) was extracted for all samples in both groups.

RESULTS: Samples hemolyzed $>0.5 \mathrm{~g} / 1:$ 12,3\% (control group). 7,9\% (intervention group). Samples hemolyzed $>1.0 \mathrm{~g} / \mathrm{l}: 4,4 \%$ (control group), 1,1\% (intervention group). Blood collection material and incidence of hemolysis: Peripheral venous catheter: 21,3\%. Butterfly needle: 2,4\%. Straight needle 1,6\%. Risk of hemolysis assessed by nurses during blood sampling correlated to observed blood flow: Slow flow: 35,9\%, fast flow $15,7 \%$, normal flow $8 \%$. Nurses positive prediction value: $26 \%$. Nurses hemolysis rate varied between $2,7 \%$ and $18,6 \%$.

CONCLUSIONS: There are a large number of identified causes for in vitro hemolysis which makes it challenging to reduce. Here we present a new method for point-of-care identification of hemolysis. In this study, the difference between the intervention group and the control group was $75 \%$ lower at $1.0 \mathrm{~g} / \mathrm{l}$ and $36 \%$ lower at $0.5 \mathrm{~g} / \mathrm{l}$.

Key Words: hemolysis; point-of-care, preanalytical

\section{THE IMPACT ON PEPTIDOMIC PROFILING OF BD BARRICOR TUBE WITH RESPECT TO PST II TUBE}

\author{
$\underline{\text { A. Padoan }}^{1}$, D. Basso ${ }^{1}$, N. Contran ${ }^{1}$, M. Zaninotto ${ }^{1}$, L. Sciacovelli ${ }^{1}$, E. Piva ${ }^{1}$, G. Arrigoni ${ }^{2}$, M. Plebani ${ }^{1}$ \\ ${ }^{1}$ Department of Laboratory Medicine, University-Hospital of Padova, Italy \\ ${ }^{2}$ Proteomic Center, University of Padova, Padova, Italy
}

BACKGROUND-AIM: Mass spectrometry (MS) analysis of plasma represents a relevant tool for biomarkers discovery. However, suboptimal centrifugation conditions of blood collection tubes, and the presence of gel separator may reduce the quality MS analyses. The aim of this study was to compare by MALDI-TOF/MS analyses the peptidomic profiles of BD Vacutainer ${ }^{\circledR}$ Barricor ${ }^{\mathrm{TM}}$ Plasma collection tube (Barricor) centrifuged at different conditions, with respect to BD PSTII plasma tube (PSTII).

METHODS: One PSTII, centrifuged at 1300gx10min, two Barricor centrifuged at 4000gx3 min and $4000 \mathrm{gx} 10 \mathrm{~min}$ and one plasma sample ultracentrifuged at $12000 \mathrm{gx} 5 \mathrm{~min}$ (Purified plasma) were collected from 29 subjects at fasting conditions. High abundant proteins were precipitated by adding to plasma acetonitrile $(1: 1, \mathrm{v} / \mathrm{v})$. Peptides were evaluated by MALDI-TOF/MS, in a mass to charge $(\mathrm{m} / \mathrm{z}) \mathrm{range}$ from 1000 to 4000. After signals median normalization, differences in features intensities were evaluated by random effect.

RESULTS: Out of 77 total MALDI-TOF/MS features identified in all plasma types, 35 features behaved differently among the studied conditions. 11/35 features intensities varied in the different plasma types with respect to the Purified Plasma condition $(p<0.05)$. Independently from centrifugation protocol, in Barricor's plasma features intensities at $m / z 1626,1638,1739,1740,1896$ varied with respect to PSTII (p<0.05). Differences in Barricor's plasma associated with the centrifugation protocol were found for features at $m / z$ 2037, 2054, 2109, 2544, 2842, 2858 $(\mathrm{p}<0.05)$. With respect to Purified plasma, the features intensities at $m / z 1598,1767,2166$ and 2228 increased progressively in Barricor centrifuged 4000gx10min, 4000gx3 min and in PSTII $(\mathrm{p}<0.05)$.

CONCLUSIONS: Barricor peptidomic profiles were more comparable with ultracentrifuged plasma than PSTII. This improvement could potentially benefit the proteomic study for the discovery of disease-associated biomarkers.

Key Words: proteomics, blood collection tubes, mass spectrometry, sample quality

\section{PRE-ANALYTICAL TRANSPORTING TIME OF INPATIENT SAMPLES: TAT OR NOT TAT? INCREASING INTRA-HOSPITAL TRACEABILITY USING THE MINI-INDEXOR® SYSTEM}

\author{
E. Costa $^{1}$, J.G. Frade ${ }^{1}$, L. Araújo ${ }^{1}$, F. Rodrigues ${ }^{1}$ \\ ${ }^{1}$ Centro Hospitalar e Universitário de Coimbra, Coimbra, Portugal
}

BACKGROUND-AIM: The pre-analytic phase is the most sensitive in the clinical laboratory activity where most errors occur, tasks are time consuming and process control is distressed by the existence of multiple procedures outside the laboratory. This complexity is increased in hospitals with inpatient units (IU) where sample traceability often begins after the arrival at the laboratory rather than in the collection areas, 
transforming the measuring of the turnaround times (TAT) in a defective task unavailable to take into account the time elapsed since the request, blood draw and transport. The authors describe how the Mini-Indexor® system (MIS, Maksense) enhances the sample traceability from de IU allowing a more rigorous assessment of the TAT, able to include the transporting time.

METHODS: Wireless MIS were incorporated into the mobile transport units (MTU) that pick routine samples from all the IU in the hospital's 10 floors. All samples were read before the transport to the laboratory and the request as the sample information sent to the LIS. A check point for the start of transport was created. Emergency areas were not considered in this 6 month evaluation. For the TAT measurement only the samples completed in the same day were evaluated.

RESULTS: A total of 60813 routine samples were read in the MTU. About 95\% were completed on the same day, with a mean transport time of 47 minutes and an overall TAT of 137 minutes.

CONCLUSIONS: Without considering the transport time the TAT is 90 minutes, however, the laboratory activity begins with the medical request and the time beyond the laboratory is waiting time and so, TAT.

The use of the MIS increased the traceability of the samples in the Hospital, allowed transport to be included in the TAT and significant gains were achieved allowing a data-supported improvement intervention in regard with sample quality and patient safety.

Key Words: traceability, Mini-Indexor, turn around time, pre-analytical phase, sample quality

\title{
ALGORITHM FOR ESTIMATION OF HEMOLYSIS INDEX IN SERUM SAMPLES.
}

$\underline{\text { O. Klimenkova }}^{2}$, T. Ivashikina ${ }^{2}$, V. Pashkova ${ }^{2}$, T. Vavilova ${ }^{1}$, V. Berestovskaya ${ }^{1}$

${ }^{1}$ Federal State Budgetary Institution «V.A. Almazov National Medical Research Center» of the Ministry of Health of the Russian Federation

${ }^{2}$ Saint Petersburg State Budgetary Institution “Advisory and Diagnostic Center for Children”

BACKGROUND-AIM: It is believed that with HI above the permissible for the analyte, which was established by the manufacturer of the analytical system, the laboratory should request a new sample. We evaluated the effect of hemolysis on the accuracy of the result of aspartate aminotransferase (AST) using the value of the critical difference RCV (Reference Change Value).

METHODS: AST results were analyzed in 41 serum samples with $\mathrm{HI}>40$. The same patients underwent repeated AST measurement at a value of HI less than 40. All studies were performed on the analyzer cobas 6000, Roche.

RESULTS: In accordance with the reference intervals (RI) in samples with hemolysis (primary) and in samples without hemolysis (after re-take) samples, the data were divided into three groups: 1- AST results in samples with hemolysis above RI, and second results within the RI;2- results of AST were higher RI in samples with and without hemolysis;3-the results of AST were within the RI in in both cases.

Clinical significance is group 1, in which HI in the sample led to false-elevated AST results (16,2\%). The number of samples assigned to groups 2 and 3 was $8.8 \%$ and $75.0 \%$, respectively. The significance of differences between between first and second measurement of AST in the case of an unacceptable HI was assessed using value RCV, which amounted to $34.2 \%$. It was found that repeated measurements of AST level in group 1 have significant differences from the primary result of $54.5 \%$, but do not go beyond RI. In the 2 group there was a significant increase of AST level in $16.7 \%$, which confirms the presence of a pathological process.

CONCLUSIONS: We believe that obtaining the AST result within RI even in the presence of hemolysis above the permissible level does not require repeated measurement. In cases where the result of the AST study in a sample with hemolysis exceeds RI, it is necessary to notify the doctor about possible interference and the need to re-take blood.

Key Words: hemolysis index (HI), reference change value, aspartate aminotransferase

\section{THE IMPACT OF DELAYED SAMPLE HANDLING ON THE INTERPRETATION OF FLOW CYTOMETRIC EXAMINATION RESULTS}

\author{
$\underline{\text { B. Kárai }}^{1}$, M. Száraz-Széles ${ }^{1}$, Z. Hevessy ${ }^{1}$, J. Kappelmayer ${ }^{1}$ \\ ${ }^{1}$ Department of Laboratory Medicine, Faculty of Medicine, University of Debrecen, Debrecen, Hungary
}

BACKGROUND-AIM: In a previous study we found that pre-analytical factors had a significant impact on the immunophenotype of cells, which can lead to post-analytical errors. By presenting 6 cases, we will demonstrate the types of effects that delayed sample handling may have on the interpretation of results. The examination of 3 of these cases focused on the evaluation of dysplastic signs, while the other 3 cases were examined in terms of minimal residual diseases (MRD).

METHODS: We performed our experiments on residual samples collected in $\mathrm{K}_{3}$-EDTA that were left over after diagnostic tests. Bone marrow samples were labelled and analyzed immediately after aspiration and up to 72 hours after sampling.

RESULTS: The fresh sample of the first patient with iron deficiency showed no dysplastic signs but it did produce ten dysplastic signs by day 2. The second patient sample indicated low-grade MDS on day 0 , and the number of dysplastic signs similarly increased by the subsequent days (dysplastic signs: $\mathrm{n}=4$ on day $0, \mathrm{n}=9$ on day 1 , and $\mathrm{n}=12$ on day 2 ). In the case of the third patient with AML, the residual normal cells showed 
dysplastic signs on day 2. The percentage of residual myeloma cells decreased by $90 \%$ each day, thus after 72 hours the samples were inadequate for the evaluation of MRD. In the case of the patient with T-ALL, the percentage of residual blasts grew from 15 to $38 \%$ after 72 hours due to the drop in the ratio of the myeloid population. In the case of the patient with B-ALL, no significant alterations could be detected. CONCLUSIONS: We observed that 5 out of the 6 cases examined demonstrate that delayed sample handling can significantly influence the interpretation of the results of flow cytometric examination. Further comprehensive studies are required to determine the exact directions of changes potentially induced by delayed sample handling.

Key Words: delayed sample handling, flow cytometry, myelodysplastic signs, minimal residual diseases

\title{
IMPLEMENTATION OF THREE KEY PERFORMANCE INDICATORS FOR THE PRE-ANALYTICAL PHASE AT OUR LABORATORY INFORMATION SYSTEM
}

\author{
M. Pastor García ${ }^{1}$, A.R. Pons Mas ${ }^{1}$, A. Ballesteros Vizoso ${ }^{1}$, P. Argente Del Castillo Rodriguez ${ }^{1}$, J. Delgado Rodriguez ${ }^{1}$, A. Rubio Alaejos ${ }^{1}$ \\ ${ }^{1}$ Hospital Universitario Son Espases, Palma de Mallorca, Spain
}

BACKGROUND-AIM: The analytical phase depends on the rapid management of the samples in the preanalytical area. In a tertiary care hospital, where a large number of samples with high complexity are processed, it is essential to have computer tools that allow us to monitor these processes. The aim of this study was to develop three key performance indicators (KPIs) to monitor the delivery time of serum samples from the preanalytical area to the core laboratory and the prompt pre-analytical detection of non-received serum samples.

METHODS: Three KPIs were computerized through a SQL query to our laboratory information system (LIS, GestLab) database to obtain the following data: serum samples with a "not received" status, sample registration trace by a sorter (AutoMate 2500, A 2500) and sample track entry trace in middleware.

RESULTS: The implementation consisted of: KPI1. Serum samples registered before $12 \mathrm{pm}$ by A 2500: monitors the delivery of primary care samples to the core laboratory. It returns the number of samples that the sorter has assigned a "received" status on LIS before this time. To optimize the work at the analytical area, we established a threshold of 600 samples. KPI2. Time from the registration of serum samples by A 2500 until its arrival at the core laboratory: It returns the time elapsed from the registration of the sample by the sorter, until its registration by the core laboratory track middleware (FlexLab). The optimal turnaround time should be 30 minutes. KPI3. Serum samples pending to be received by A 2500. It returns the serum sample identification with "not received" status on the LIS.

CONCLUSIONS: These KPI optimize laboratory workflows and avoid delays in result generation. They are especially useful to ensure compliance with the turnaround time agreed with hospital departments. It is necessary to monitor their use and establish optimized cut-offs in terms of number of samples and delivery times to the core laboratory for continuous improvement.

Key Words: blood tubes, local validation, blood collection, venipuncture, errors

\section{DEVELOPMENT AND IMPLEMENTATION OF AN ALERT SYSTEM TO ASSURE A CORRECT TUBE TRANSPORT TEMPERATURE USING THE AUTOMATED PROTUBE DEVICE}

\author{
$\underline{\text { M. Pelloso }}^{1}$, E. Piva ${ }^{1}$, F. Tosato ${ }^{1}$, M. Marinova ${ }^{1}$, A. Aita ${ }^{1}$, L. Sciacovelli ${ }^{1}$, A. Padoan ${ }^{1}$, M. Plebani ${ }^{1}$ \\ ${ }^{1}$ Department of Laboratory Medicine, University-Hospital of Padova, Italy
}

BACKGROUND-AIM: Some of the preanalytical errors are attributable to incorrect temperature conditions during tube transport, particularly in laboratory testing for cryoglobulin or ammonium. To obviate these errors during phlebotomy, an automated approach such as pop-up or alert for specific samples requirements should be used. We have implemented an automated alert system in the ProTube device to remind the phlebotomist of the particular transportation conditions needed for special samples.

METHODS: In the phlebotomy process, the ProTube device (Inpeco, Lugano, Switzerland) is used for operating and labeling blood test-tubes and other biological material containers, providing automation in test order examination, patient identification, tubes and/or container specimen selection and labeling. In an outpatient setting, before and after implementation of the ProTube alert system, the rate of preanalytical errors due to incorrect transport temperature was evaluated in all samples along with laboratory testing for cryoglobulin or ammonium. RESULTS: The ProTube alerts were evaluated for 12 months in 764 outpatients undergoing phlebotomy, of which 446 required cryoglobulin determination, while 318 required ammonium testing. Results are compared against those of the 12 months before (539 and 280 respectively). Due to incorrect transportation temperature the rate of unsuitable samples was for samples at $37^{\circ} \mathrm{C}: 0,037(20 / 539)$ without alert system and $0,002(1 / 446)$ after alert system implementation $(\mathrm{P}=0,0002)$, while for samples at $4{ }^{\circ} \mathrm{C}+2{ }^{\circ} \mathrm{C}: 0,036(10 / 280)$ without alert system and 0,0 $(0 / 318)$ after alert system implementation $(\mathrm{P}=0,0007)$.

CONCLUSIONS: The results obtained in this study attest that the introduction of automated alerts using the ProTube improves the reduction of some preanalytical errors related to maintaining the testing temperature condition. By decreasing these types of preanalytical errors we avoid the need to recall outpatients, thus significantly improving the preanalytical process in our phlebotomy center.

Key Words: phlebotomy, automation, automated alerts 


\title{
WHEN LESS IS MORE ON SAMPLE TRANSPORT - THE CASE FOR A SMART CONTAINER
}

\author{
V. Lopes Dos Santos ${ }^{1}$, I. Cachapuz Guerra ${ }^{1}$ \\ ${ }^{1}$ CoreLab, Clinical Pathology Service, Pedro Hispano Hospital, Local Health Unit of Matosinhos, Matosinhos, Portugal
}

BACKGROUND-AIM: Centralization of Labs is a reality, and the samples transportation also became increasingly important. The control of transport temperature and packing must be very thorough, in accordance with international standards. (UN3373). The current solutions in temperature and impact control require the use of several components (cooler, data loggers, etc.). This amount of material might hamper the process. With this new Radio-Frequency-Identification technology (RFID), placed in coolers, we intend to provide monitorization of the transport of samples and prevent any incidents.

METHODS: iLogger Case 4A® (IC4A) was built to end with all this diversity, complying with the demanded standards (UN3373), and complementing the Indexor ${ }^{\circledR}$ solution which is already installed in the Service of Clinical Pathology (SCP). IC4A registers the temperature conditions and impacts directly into the transportation racks RFID and this information is then sent to Indexor®, which produces an automatic report of the transport conditions and alerts users for any incidents. Thus, information will remain linked to the sample it belongs to. We carried out a study for nine months, having controlled a grand total of 26248 samples.

RESULTS: IC4A allows for better monetization of professionals while at work, so they can dedicate their time to report analysis and performance of corrective actions, as they have live information upon arrival of samples. Before, professionals spent a huge amount of time manually producing reports with nothing but the temperature conditions upon transportation and no possibility of including any specifics attached to the sample itself.

CONCLUSIONS: Thanks to IC4, SCP now has an adequate temperature and impact control system, which allow corrective actions to be taken in real time, much faster and efficiently and very considerate to the SCP quality continuous improvement, focusing on the patient's safety.

Key Words: control, temperature, impact, UN3373, patient safety

\section{SIMPLIFYING WHILE STANDARDIZING - IMPLEMENTATION OF AN AUTOMATIC CHECK-IN METHOD}

\author{
V. Lopes Dos Santos ${ }^{1}$, V. Cachapuz Guerra ${ }^{1}$ \\ ${ }^{1}$ CoreLab, Clinical Pathology Service, Pedro Hispano Hospital, Local Health Unit of Matosinhos, Matosinhos, Portugal
}

BACKGROUND-AIM: Preanalytical phase is complex, with many players involved, a lot of manual handling and no standardized processes, making it more prone to errors - accounting for $70 \%$ of errors of the Total Testing Process. In spite of all automatic solutions, barcode reading tube-by-tube is still very common on sample arrival at the Central Laboratory (CL). We aim to evaluate the use of RFID technology and barcode reading upon sample collection, to allow for automatic Check-in (CI).

METHODS: A mini Indexor ${ }^{\circledR}$ at collection stations registers the sample information (collection time and date, position, Phlebotomist) into a rack RFID. Transport container iLogger Case A4 (LC4A) adds information on temperature conditions and impacts, case identification and courier identification. An Indexor ${ }^{\circledR}$ system at the Core Lab, enabling all the previous data to be automatically read from the RFID and sent to the Laboratory's Information System (LIS). All samples are automatically checked-in at the LIS and urgent samples are immediately identified and prioritized. 232.660 samples were checked-in following this method for 9 months.

RESULTS: After 9 months, standardization of the process was notable as the time for Check-in took noticeably less. The new method took only 8 minutes (on average) to fully process and release samples for testing, with inter and intra $\mathrm{CV}=0,9$. Furthermore, all urgent samples were released only about 5 minutes after $\mathrm{Cl}$, with a $\mathrm{CV}$ of 1,5. The Indexor ${ }^{\circledR}$ solution allowed for automatization and standardization upon arrival of sample to Central Laboratory (CI), using data obtained in collection. Before, CI process was highly variable, both in time and user work methodology, ranging from 1 to 2 hours.

CONCLUSIONS: Implementation of automatic Cl proved possible the simplification of this critical step in the PA phase, dramatically reducing time and introducing standardization with proper urgent samples prioritization.

Key Words: check-in, preanalytic, standardization, methodology

\section{FORMALIN FIXED PARAFFIN EMBEDDED (FFPE) TISSUE SAMPLES - ARE THEY GOOD ENOUGH FOR ROUTINE MOLECULAR CLONALITY TESTING?}

\author{
M.M. Kardum Paro ${ }^{1}$, Z. Šiftar ${ }^{1}$, I. Bašić ${ }^{1}$, A. Škrtić ${ }^{2}$, S. Gašparov ${ }^{2}$ \\ ${ }^{1}$ Department of Medical Biochemistry and Laboratory Medicine, Merkur University Hospital, Zagreb, Croatia \\ ${ }^{2}$ Department of Pathology, Merkur University Hospital, School of Medicine University of Zagreb, Zagreb, Croatia
}

BACKGROUND-AIM: The diagnostic material for hematopathology, where the special tool is molecular clonality testing by polymerase chain reaction (PCR), almost exclusively consists of formalin fixed paraffin embedded (FFPE) tissue samples. Although there is better DNA quality 
in fresh tissues, getting DNA from FFPE tissues is still a challenge. The aim was the quality assessment of DNAs extracted from FFPE tissue samples for molecular clonality testing.

METHODS: DNAs were extracted from 66 FFPE tissue samples by proteinase K digestion followed by alcohol precipitation. The quality assessment of extracted DNAs was performed by the BIOMED-2 multiplex PCR method resulting in a ladder of PCR fragments (100, 200, 300, 400 and 600) in base pairs (bp). The extracted undiluted and diluted DNAs (1:5) were amplified with Control Size Ladder (CSL) master mix and analyzed by agarose gel electrophoresis. The size of PCR fragment was an ultimate parameter for the correct interpretation of the results and for final conclusion. DNA of adequate quality was considered when PCR fragment size $\geq 200$ bp was obtained and heavily degradated DNA when PCR fragment size was below $200 \mathrm{bp}$.

RESULTS: During two years 66 DNAs were extracted from FFPE tissue samples. Most of them were of adequate quality with PCR fragment size $\geq 200$ bp (61/66, 92,4\%). Heavily degradated DNAs were only few (5/66, 7,6\%). Amplified PCR fragments of max 400 bp was the upper limit for DNAs from FFPE tissue samples (21/61, 34,4\%), those of $300 \mathrm{bp}$ were considered as very good quality DNAs (26/61, 42,6\%) and amplified PCR fragments of $200 \mathrm{bp}$ as good quality DNAs $(12 / 61,19,7 \%)$ that could be used for further molecular clonality testing.

CONCLUSIONS: The protocol for DNA extraction from FFPE tissue samples was used appropriately and DNAs extracted from FFPE tissue samples were succesfully amplified. BIOMED-2 control gene PCR method showed as an objective and reliable tool for the quality assessment, but also essential for routine molecular clonality testing.

Key Words: FFPE tissue samples, DNA extraction, molecular clonality testing

\title{
REAL TIME PREANALYTICAL INCIDENCES RESOLUTION THROUGH LIMS LOGBOOK: NO MORE "SAMPLES NOT RECEIVED".
}

\author{
$\underline{\text { A. Pozo-Giráldez }}{ }^{1}$, A. Corchón-Peyrallo ${ }^{1}$, M. Díaz-Giménez ${ }^{1}$, C. Quiñones-Torrelo ${ }^{1}$, E. Rodríguez-Borja ${ }^{1}$ \\ ${ }^{1}$ Hospital Clínico Universitario de Valencia, Spain
}

BACKGROUND-AIM: Historically, registration of preanalytical incidences was limited to the existence of some automatic comments in the final report with the aim of notifying the incidence through a simple default text. Infrequently, all these data were employed in order to enhance preanalytical processes by means of a continuous improvement cycle or even to solve incidences in a minimum time. Our main goal was to implement in our LIMS a funcionality that allow us to: 1) Receive and coordinate all the preanalytical incidences coming from phlebotomy centers and/or intralaboratory areas. 2) Release of personalised corrective actions according to incidencés type. 3) Claim of new samples via electronic means to phlebotomy centers. 4) Incidence resolution and final report.

METHODS: An internal tool for incidences register, monitoring and resolution was developed in our LIMS (Gestlab 8.0 (c) Cointec) in July 2011 based on incidences/corrective actions generation system for each request. Incidences and actions would be monitored in a new logbook. Incidences could be created by intralab areas and phlebotomy centers users (e.g. sample not received). These incidences were centralized by Laboratory Quality Area (QA) professionals who were in charge of their resolution through corrective actions attached to incidences (e.g. claim a new sample through an email to phlebotomy center and hold internally related tests for worklists). Incidences were resolved and reported within a maximum period of 15 days by QA. An statistical quality control has been performed twice a year since its implementation regarding samples claimed/recovered rate.

RESULTS: The number of samples claimed have been progressively decreasing since $20112^{\text {nd }}$ semester to $20181^{\text {st }}$ semester (1553 vs. 238 ). Additionally, the rate of samples recovered has experienced an outstanding increase since $20112^{\text {nd }}$ semester (33,7\%) vs. 2018 $1^{\text {st }}$ semester (79,4\%).

CONCLUSIONS: Software implementation has led to a notorious improvement in preanalytical quality regarding samples not received.

Key Words: preanalytical quality control, preanalytical reports, primary care centers, continuous improvement circuit

\section{STANDARDISATION OF TOTAL RNA EXTRACTION MANUALLY FROM HUMAN VISCERAL ADIPOSE TISSUE - MODIFYING TRIZOL-TO-TISSUE RATIO IS KEY IN ELIMINATING INTERFERENCES}

\author{
D. Roy ${ }^{1}$, A. Modi ${ }^{1}$, S. Tomo ${ }^{1}$, P. Purohit ${ }^{1}$, P. Sharma ${ }^{1}$ \\ ${ }^{1}$ Department of Biochemistry, All India Institute of Medical Sciences (AIIMS), Jodhpur
}

BACKGROUND-AIM: Extraction of RNA from human adipose tissue poses a multitude of challenges such as scanty tissue sample, high lipid content, difficulty in phase separation, lower yield and existing impurities. To our current knowledge, there are few human studies giving satisfactory data on manual RNA isolation from human adipose tissue. The primary aim of this study was to assess the role of a varying trizol ratio and an additional chloroform wash in the existing extraction protocol.

METHODS: We subjected a total of 14 human visceral adipose tissue samples for RNA extraction using HiMedia RNA-XPress Reagent. 
RESULTS: By varying the amount of trizol in three different groups (Trizol [in (L] to Tissue [in mg] in ratios of 4:1, 2:1 and 2:3 respectively), the absorbance ratio improved gradually in the latter two groups compared to the first for both 260/280 (Mean \pm SD 1.77 $\pm 0.24,1.87 \pm 0.33$ and $1.93 \pm 0.13$ respectively) and 260/230 (Mean \pm SD $0.23 \pm 0.12,0.63 \pm 0.42$ and $1.01 \pm 0.52$ respectively) and the improvement of 260/230 ratio with decreased trizol was statistically significant between the first and the third groups $(\mathrm{p}=0.0223)$. Again, on adding a second step of chloroform wash to the RNA extraction protocol, we found that there was a significant decrease in the RNA yield (in ng/ ( L) (24.68 \pm 7.75 compared to $87.62 \pm 15.11$ with a single wash; $p=0.0038)$; also, the absorbance ratio of $260 / 230$ deteriorated significantly $(0.32 \pm 0.19$ compared to $1.23 \pm 0.22$; $\mathrm{p}=0.0001)$ while there was a non-significant decrease in $260 / 280(p=0.86)$. Increasing the quantity of tissue sample as well as overnight incubation before the chloroform step didn't show any noticeable changes in the absorbance ratios.

CONCLUSIONS: Our study showed that on modification of the manual method of RNA extraction by decreasing the amount of trizol used (in (l) per mg of tissue (in a 2:3 ratio) and a single chloroform wash, there was a significant improvement in the quantity of RNA and absorbance ratios.

Key Words: total RNA extraction, adipose tissue, Trizol method, RNA purity

\title{
ROBUSTNESS OF BLOOD COLLECTION TUBES WIH RNA STABILIZERS
}

\author{
G. Hamot ${ }^{1}$, C. Stellino ${ }^{1}$, C. Bellora ${ }^{1}$, J. Trouet ${ }^{1}$, F. Betsou ${ }^{1}$ \\ ${ }^{1}$ Integrated BioBank of Luxembourg, Luxembourg
}

BACKGROUND-AIM: RNA gene expression profiles change quickly after blood collection, efficient stabilization of the blood specimen is therefore key to obtain reliable and comparable RNA analysis data. PAXgene (Qiagen - Beckton Dickinson) and Tempus (Applied Biosystems - Life Technologies) Blood collection tubes with RNA stabilisers avoid both preanalytical degradation of mRNA by endogenous nucleases and preanalytical modifications in specific mRNA concentrations by unintentional up- or down-regulation of gene expression.

METHODS: A total of 16 different preanalytical conditions were tested in blood samples from 7 donors. Blood was collected in PAXgene and Tempus tubes, and subjected to the following preanalytical variations: different mixing after collection, different fill volumes, different $24 \mathrm{hr}$ transport temperature conditions after collection and different RNA extraction methods. The quality attributes of the extracted RNA were assessed by spectrophotometric quantification, A260/A280 purity ratio, RNA Integrity Number (Agilent BioAnalyser), miRNA qRT-PCR on two target miRNAs (RNU-24 and miR-16), mRNA Quality Index by RT-qPCR on the 3' and 5' region of the GAPDH gene, and PBMC Preanalytical Score, based on the relative expression levels of the IL8 and EDEM3 coding genes.

RESULTS: When Paxgene RNA and Tempus blood collection tubes were used following the manufacturers' instructions, there was no statistically significant difference in the output RNA quality attributes. However, the Tempus collection tubes were less robust, with the total RNA integrity being significantly affected by preanalytical variations that were deliberately applied. The PAXgene system showed susceptibility to fewer preanalytical variations than Tempus, but when an impact occurred, it was of greater magnitude.

CONCLUSIONS: Blood collection tubes with different RNA stabilisers present different robustness to common preanalytical variations. This information on differential robustness should be included in the validation of the method.

Key Words: preanayltical phase

\section{IS SAMPLE MIXING NECESSARY PRIOR TO HBA1C MEASUREMENT ON THE BIORAD D-10 ANALYZER?}

\author{
$\underline{\text { I. Brkić }}^{1}$, A. Kranjčina ${ }^{1}$, J. Starčić ${ }^{1}$, M. Žarak ${ }^{1}$, S. Škorvaga ${ }^{1}$, N. Stančin ${ }^{1}$, M. Živković ${ }^{1}$ \\ ${ }^{1}$ Clinical Department for Laboratory Diagnostics, Clinical Hospital Dubrava, Zagreb, Croatia
}

BACKGROUND-AIM: HbA1c values are an important indicator of patient's glycemic control and diagnosis of diabetes mellitus. Our HPLC analyzer BioRad D-10 measures HbA1c in whole blood, a maximum of 10 samples in series. Aim of this study was to determine whether sample mixing affects HbA1c measurement, since the analyzer does not mix the samples and the analysis of a full series takes 30 minutes.

METHODS: HbA1c values of 30 samples (K EDTA anticoagulant, BD Vacutainer) were mixed and measured routinely (reference measurement, R) on the analyzer BioRad D-10 (BioRad Laboratories, Hercules, USA). HbA1c was then measured after 30 and 60 minutes (first, M1, and second, M2 measurement) of sedimentation, without mixing prior to analysis. The data was tested for normality with Kolmogorov-Smirnov test and differences were tested with t-test for paired samples.

RESULTS: Measured values of HbA1c ranged from 4,3\% to $11,2 \%$. All three measurement groups were normally distributed, with arithmetic means and standard deviations as follows, R: 6,3\% $\pm 1,2 \%$; M1: 6,2\% $\pm 1,2 \%$ and M2: 6,2\% $\pm 1,2 \%$. Obtained P-values were found statistically significant between the reference and first measurement $(\mathrm{P}=0,009)$ and reference and second measurement $(\mathrm{P}<0,001)$. Also, since M1 and M2 measurements are compared to the initial, reference measurement, relative bias values were calculated between R and M1 and R and M2. The mean bias values were 1,5\% for R and M1, and 1,3\% for R and M2 measurements. 
CONCLUSIONS: There was a statistically significant difference between the reference measurement and the measurements without sample mixing. However, obtained bias values are within the desirable biological variation criteria for bias (1,5\%). In conclusion, sample mixing prior to $\mathrm{HbA} 1 \mathrm{c}$ measurement on the BioRad D-10 analyzer is necessary to obtain an accurate and clinically relevant HbA1c value.

Key Words: HbA1c measurement, diabetes mellitus, sample mixing, HPLC

\title{
IMPLEMENTATION OF A NEW MONITORING SYSTEM FOR TRANSPORT TEMPERATURE
}

\author{
M.d.S. López Velez ${ }^{2}$, M.T. González Cejudo ${ }^{2}$, L. Papay Ramírez ${ }^{1}, \underline{T}^{\text {. De Haro Muñoz }}{ }^{2}$ \\ ${ }^{1} B D$ Life Sciences, PAS \\ ${ }^{2}$ Hospital Universitario San Cecilio, Granada, Spain
}

BACKGROUND-AIM: All laboratory activities may be subject to errors, but a large proportion occurs in the pre-analytical phase where human participation is maximal. The ISO15189 says that laboratory is the responsible for ensuring the transport of samples in optimal conditions of time and temperature. The aim in this study is to evaluate the implementation of a new system for monitoring the temperature of biological samples transportation.

METHODS: A study is carried about processes involved in the samples road from the different extraction points to the reception laboratory. We establish rejection criteria to ensure the quality of samples after transportation. A new monitoring system with RFID developed by BD is installed in the reception area from the laboratory. A sensor is placed in each fridge container to measure the temperature every 10 min. A specific software collects automatically the data for 15 days without user intervention. We evaluate the correct identification, download data and optimal transport conditions of each container (objective criteria). We make a questionnaire to know the speed and easy collect data and satisfaction for user (subjective criteria).

RESULTS: Laboratory receives a total of 6000 samples daily from 104 extraction points. They arrive in 52 transport containers. For the rejection criteria established, no sample is rejected and the containers identification is $100 \%$. In $98 \%$ of cases data download is correct. The remaining $2 \%$ corresponds to identified and resolved causes. The questionnaire is done to 25 carries, 5 laboratory technicians and 2 clinicians. The satisfaction degree is $100 \%$.

CONCLUSIONS: To reach the highest quality standards is necessary to carry out a strict control of the transport conditions of the samples. The automation of a system for measuring and downloading data without user intervention minimizes the errors that occur in this process while facilitating the work of carriers and laboratory staff.

Key Words: transport temperature, monitoring, ISO 15189, preanalytical variables

\section{IMPROVING SAMPLE TRACEABILITY IMPROVES SAMPLE DELIVERY - A HOSPITAL CASE}

\author{
J.G. Frade ${ }^{1}$, E. Costa $^{1}$, L. Araújo ${ }^{1}$, F. Rodrigues ${ }^{1}$ \\ ${ }^{1}$ Clinical Pathology Department, Centro Hospitalar e Universitário de Coimbra, Portugal
}

BACKGROUND-AIM: Sample traceability is key in Clinical Laboratories. At Coimbra University Hospital Centre, sample delivery is achieved by 1) a pneumatic tube system (PTS), requiring permanent technician intervention to collect emergency/priority samples from delivery capsules; or 2) manual pick-up (MP-U) at predefined time periods, for routine samples sent by Wards (with batch delivery to the Laboratory). As despite previous improvements an intensive manual work was still being required to collect samples from PTS capsules, we used a newly implemented Mini-Indexor ${ }^{\circledR}$ tracking system (MIS, Maksense, Portugal) to determine if sample delivery could be improved even further.

METHODS: Samples delivered to the Laboratory were recorded in MIS barcode readers connected wirelessly to LIS, placed 1) at the pneumatic tube system station (P.-1) or 2) in MP-U crew cars, allowing samples to be checked-in automatically without further human intervention. PTS and MP-U deliveries were characterized after one month and compared in order to determine optimization opportunities.

RESULTS: During April/2018, hourly distribution of 8847 samples revealed a peak delivery by PTS between 7-8am, corresponding to 2-3 times more the number of samples delivered at the second-highest time period (4602, 9-11am). No additional peaks of operation were observed for PTS. Regarding MP-U, recordings showed a uniform recollection of biological samples according to pick-up schedule. Samples from the Intensive Care Unit, Surgical Intermediate Care Unit and Hematology, Nephrology, Cardiology and Hepatic Transplantation Wards were responsible for $67.8 \%$ of PTS deliveries (6005 samples). This was determined to arise for convenience according to ward routine and not patient status and was observed in subsequent months.

CONCLUSIONS: Improving sample traceability at the pre-analytical phase revealed hidden opportunities for improvement, potentially leading to the optimized sample delivery and processing.

Key Words: sample delivery, pneumatic tube system, sample traceability 


\title{
Preanalytical quality management
}

\section{IS IT TRUE HYPOPARATHYROIDISM? AN INVESTIGATION OF PRE-ANALYTICAL ISSUES IN THE MEASUREMENT OF INTACT PARATHYROID HORMONE FOR CONTINUOUS QUALITY IMPROVEMENT}

\author{
$\underline{\text { S. Ahmed }}^{1}$, L. Jafri ${ }^{1}$, I. Siddiqui ${ }^{1}$ \\ ${ }^{1}$ Section of Clinical Chemistry, Department of Pathology and Laboratory Medicine,The Aga Khan University Karachi, Pakistan
}

BACKGROUND-AIM: Intact Parathyroid hormone (iPTH) has a short half-life i.e. 5 mins therefore the sampling regimen has to pass through a stringent pre analytical process control. Noncompliance may lead to falsely low results. While signing out Lab reports the Clinical Chemistry faculty was encountering an increasing number of iPTH results that were below the reference interval and not correlating with other laboratory parameters and clinical details. The aim of this audit was to identify the causes of apparently falsely low iPTH from July to December 2017.

METHODS: This study was conducted at the section of Clinical Chemistry, The Aga Khan University Hospital (AKUH) Karachi Pakistan. AKUH Clinical lab has more than 10 stat labs and 264 phlebotomy stations across Pakistan. Audit tool utilized was Plan-Do-Check-Act Cycle. After correlating with available clinical details and lab parameters, telephonic history was taken for all low iPTH ( $<16 \mathrm{pg} / \mathrm{ml})$. A fresh sample was requested to confirm the results for non-correlating cases. Based on the findings appropriate interventions were undertaken and re audit was done from January to March 2018.

RESULTS: During the study period 2559 iPTH samples were analyzed. 110 (4.3\%) were identified as apparently falsely low. On analysis of fresh sample under instructions of immediate centrifugation and cold chain maintenance, 60 (2.4\%) were found to have normal or elevated levels. The causes identified were noncompliance of staff with pre analytical steps including delayed sample separation and unfavorable temperature chain maintenance. Interventions included online lectures for the staff country wide and circulation of flyers detailing the preanalytical steps via emails and hard copies. Re-audit showed reduction in number of apparently falsely low results to 30(2.1\%), out of a total of 1448 samples. Reduction in rate of change in report to $18(1.2 \%)$ was achieved.

CONCLUSIONS: Stringent pre-analytical process control is vital for quality reporting and patient safety.

Key Words: parathyroid hormone, pre-analytical phase

\section{COORDINATION PRIMARY HEALTH CARE / SPECIALIZED ATTENTION IN THE CLINICAL LABORATORIES OF SAN JORGE GENERAL HOSPITAL (CLSJH) IN HUESCA (SPAIN)}

\author{
J. Arribas Garcia $^{2}$, A. García Claver ${ }^{1}$, A. Betrán Escartín ${ }^{2}$, M. Mairal Claver ${ }^{2}$, A. Bellés Bellés ${ }^{3}$, A. Lopez Villa ${ }^{2}$, A. Luis Gracia ${ }^{2}$, M.T. Serrano \\ Fernandez ${ }^{2}$, A. Milagro Beamonte ${ }^{4}$, L. Torres Sopena ${ }^{2}$ \\ ${ }^{1}$ Clinical Analysis Laboratory, Hospital General San Jorge, Huesca, Spain \\ ${ }^{2}$ Microbiology and Parasitology Laboratory, Hospital General San Jorge, Huesca, Spain \\ ${ }^{3}$ Microbiology and Parasitology Service, Hospital Universitari Arnau de Vilanova, Lleida, Spain \\ ${ }^{4}$ Microbiology and Parasitology Service, Hospital Universitario Miguel Servet, Zaragoza, Spain
}

BACKGROUND-AIM: In the CLSJH a Quality Management System based on ISO 15189 is implemented. This system requires and promotes the continuous improvement of the processes that take place in the CLSJH, most of whom start in Primary Health Care Units (PHCU). Preanalytical phase is the most difficult process to control due to the physical remoteness of the Laboratories' sampling centers. All CLSJH provided diagnostics depend on the quality of the sample received. A mishandling, poorly transported or inadequate storiage conditions, can lead to misdiagnosis and inadequate treatment.

METHODS: The improvement of this phase is based on the coordination between the CLSJH and PHCU, mainly the personnel related to collection and reception of samples. Collaboration is based on two main points: annual PHCU self-evaluation: including a self-report sent to the CL and audits carried out by the Quality Coordinator of Primary Health Care and Quality Managers of the CLSJH, to verify that the PHCU apply the procedures elaborated by the laboratories themselves, and that they have at their disposal all documents published on the intranet of Aragon Public Health Care System. These audits are carried out with a maximum frequency of 3 years in each sampling center.

RESULTS: To improve these needs and having the feedback opportunity, the CLSJH has developed workshops on: preanalytical procedures, selection, identification and labeling of the different sample containers and blood collection tubes to the professionals of PHCU and, additionally, a poster was created, where the containers and tubes necessary for each determination are visually reflected so that it is available in the extraction rooms.

CONCLUSIONS: These actions have facilitated an increase in the exchange of information with Primary Health Care professionals that has allowed identifying opportunities for improvement in the preanalytical phase, especially focusing on topics as samples reception, samples collection and recording of necessary data

Key Words: workshop, primary health care, samples collection 


\title{
RISK MANAGEMENT: AN EXAMPLE OF APPLICATION ON SAMPLE TRANSPORTATION AND RECEPTION PHASES
}

\author{
$\underline{\text { A. Aita }}^{2}$, A. Padoan ${ }^{2}$, G. Antonelli ${ }^{2}$, L. Sciacovelli ${ }^{1}$, M. Plebani ${ }^{3}$ \\ ${ }^{1}$ Department of Laboratory Medicine, University-Hospital, Padova, Italy \\ ${ }^{2}$ Department of Medicine-DIMED, University of Padova, Italy \\ ${ }^{3}$ Department of Medicine-DIMED, University of Padova, Italy and Department of Laboratory Medicine, University-Hospital, Padova, Italy
}

BACKGROUND-AIM: Excessive temperature conditions and transport time affect sample quality. According to ISO 15189:2012, laboratory should monitor these parameters to guarantee sample suitability thus patient safety. Due to the failure to deliver a container with many samples within the defined time (May 2017), the Laboratory of Padua applied the risk management principles to correct and prevent failures occurring in ongoing processes.

METHODS: According to CLSI EP18-A2, Failure Reporting and Corrective Action System (FRACAS) was used to identify and analyse causes of errors occurred in sample transportation and reception so that additional control measures can be designed. Risk index (RI) was calculated, before and after, by multiplying errors occurrence, detectability and severity scores (ISO 14971:2012 scales).

RESULTS: 3266 transports, arrived at laboratory between January and May 2017, were analysed. The total percentage of errors was $8.8 \%$, due to containers: not delivered within the defined time (2), partially emptied (5), erroneously sent to other locations (15) and untraceable because of the lacking activation of the device recording transport data (266). The overall RI associated to the studied processes was equal to 18 . The awareness-raising of staff on the importance of using the available operating instruction to ensure patient safety, the identification of a more comfortable sample reception space, the signature upon receipt and the implementation of an app on lab staff phones improved the capability to intercept failure before they occur. RI calculated six months later was equal to 9.

CONCLUSIONS: The application of risk management principles to correct and prevent failures improved the entire process studied. The systematic application of risk management principles and implementation of evidence-based practice for tackling system failures can be considered the best solution to ensure quality in healthcare and improve patient safety.

Key Words: preanalytical phase

\section{APPROPIATENESS OF TEST REQUEST FOR STOOL PARASITE TESTING. A TOOL IN LABORATORY MANAGEMENT}

\author{
M. Garcia-Gonzalez ${ }^{1}$, A. Bellés ${ }^{1}$, A. Bernet ${ }^{1}$, J. Aramburu ${ }^{1}$, N. Aixalà ${ }^{1}$, M.P. Recuero ${ }^{1}$, A. Manonelles ${ }^{1}$, M. Ibarz ${ }^{1}$ \\ ${ }^{1}$ Sección de Microbiologia. Laboratorio clínico ICS Lleida. IRB Lleida, Spain
}

BACKGROUND-AIM: The microscopic examination is the traditional method for stool parasite testing. This is labour-intensive and requires a high level of skill for optimal examination. At present, there are several PCR-based tests with a high sensibility, but also more expensive. The aim of this study was to assess the clinical appropriateness of the requests to ensure the efficiency of PCR-based tests introduction in our lab. METHODS: We retrospectively revised 2 periods of 6 months (2012 and 2018), from the 1st January to the 30th June in both cases. Data were obtained from informatics lab system (Modulab $₫$, Werfen $\AA$ ). We considered adults $>15$ years old inclusive. We revised if related-clinical information was provided when ordered a parasitic analysis, with information about patients characteristics as healthy or immunocompromised, clinical signs like anorexia, weight loss, exposure risks, elevated IgE, urticaria, proglotids or worms noticed by patient. Between those periods we have continuously informed requesting doctors to improve the preanalytical phase

RESULTS: 2101 (in 2012) and 2849 (in 2018) requests were revised, being 35.4\% increment between the two periods. Adults(\%), Average age (adults/children) and Sex (\%female) were: in 2012: 46\%; 44/6 years old; 53\%; in 2018: 64\%; $47 / 7$ years old; 52\%. Clinical diagnosis provided: 2012, Blank space 465 ; Non-related diagnosis 562; Related diagnosis 1064; Travel history 10. 2018, Blank space 386; Non-related diagnosis 1016; Related diagnosis 1441; Travel history 9.

CONCLUSIONS: No differences in age or sex were detected. Only half of the requests showed related clinical information without changes along years. As we need physician guidance tool to aid in appropriate ordering tests, according to our results we have decided to implement the new diagnostic methods only in case demand managing is done.

Key Words: parasites, parasitic analysis, demand managing

\section{HOW TO REDUCE YOUR SAMPLE HAEMOLYSIS RATE - GUARANTEED}

\author{
R. Hawkins ${ }^{1}$ \\ ${ }^{1}$ Laboratory Medicine, Tan Tock Seng Hospital, Singapore
}

BACKGROUND-AIM: Sample haemolysis assessment by automated spectrophotometry is a routine procedure in many clinical laboratories, allowing easy assessment of haemolysis rates by location and providing data for sample quality improvement monitoring. We 
describe the effect on our perceived sample haemolysis rates by changing from the Beckman Coulter DxC-800 to the AU-5800 as our analytical platform.

METHODS: From 2005 to mid 2018, our main chemistry analytical system was a Beckman Coulter Power Processor system with 3 DxC-800 clinical chemistry analysers. We routinely performed haemolysis index measurement on all specimens and used the results to filter analytical results as well as for phlebotomy quality improvement initiatives. In May 20182018 we changed to a Beckman Coulter Power Express, with 2 AU-5800 clinical chemistry analysers. The effect of the analyser change on reported haemolysis rates was assessed by extracting haemolysis index results from the laboratory information system for 2018 and the 3 earlier years.

RESULTS: The hospital wide haemolysis rates for the months of Q3 (July, Aug, Sept) were 2015: 18.1\%, 18.7\%, 18.9\%; 2016: 18.7\%, 19.9\%, 19.0\%; 2017 18.0\%, 17.6\%, 16.7\%; 2018: 3.2\%, 3.35, 3.4\%. This reduction is seen across all sites - the range of monthly haemolysis rates for Jan-April 2018 vs June-Sept 2018 were: ED ICU 26.6-33.8\% vs 9.1-10.45; ICU 17.3-19.3\% vs 1.2-4.0\%; Inpatients 20.2-22.8\% vs 2.4-3.7\%; Outpatients 7.6-9.8\% vs 0-2.3\%.

CONCLUSIONS: According to Beckman Coulter, the DxC-800 will measure a haemolysis index of 1-2 at 0-75 mg/dL haemoglobin while the AU-5800 will measure haemolysis as absent at $<50 \mathrm{mg} / \mathrm{dL}$ and $1+$ at 50-99 mg/dL. By changing to an analyser with a higher cut-off for haemolysis detection, our perceived haemolysis rates have automatically dropped. Laboratories should be aware of inter-instrument differences in serum index cut-offs, even in instruments from the same manufacturer.

Key Words: haemolysis, serum indices, phlebotomy

\title{
RISK ERRORS ASSESSMENT DURING THE SAMPLES JOURNEY TO THE LAB: THE ROLE OF THE PRE-ANALYTIC STAGE
}

\author{
J. Attias $^{1}$, M. Mekel ${ }^{2}$, M. Kranzler ${ }^{2}$, M. Kaplan ${ }^{1}$ \\ ${ }^{1}$ Laboratory Department,Rambam Health Care Campus, Haifah Israel \\ ${ }^{2}$ Risk Management Unit, Rambam Health Care Campus, Haifa, Israel
}

BACKGROUND-AIM: About 65\% of errors occurs in the pre analytic phase. Cancellation of samples, due to non-compliance to pre analytics requirements, is a major issue. The clinical implications are quite extensive and include a substantial increase in the turnaround time of the test required, a distress for the patients since a new sample is required. Patients identification error is the issue with the higher risk potential toward the patients. Aims: To collect data on the laboratory cancellation resulting from pre-analytics issues in our medical center, focusing on patient identifications errors in order to assess the extent of this issue, to identify the wards with the highest level of patient's identification errors and finally to propose an intervention plan to reduce the risk.

METHODS: Use of failure mode and effect analysis method (FMEA method) to evaluate the risk hidden in every stage of the pre analytic stage during the sample journey and the chance to identify the errors.

RESULTS: Our results demonstrate that the major reasons for samples cancellations are patient's identification problems and hemolysis (30\% and $32.7 \%$ of the cancelled samples, respectively). Use of the FMEA model via an external quality assurance program demonstrate that our risk percentage as a result of identification incidents is significantly higher comparatively to the other participants (14.85\% and 5.09\% respectively) and similar to the results of the group for sample hemolysis. The internals and emergency departments are in the top list of laboratory tests cancellation due to identification problems ( $28 \%$ and $15 \%$ respectively).

CONCLUSIONS: An intervention plan was set up towards internal and emergency departments including data reports to relevant wards directors, standardization of procedures for samples cancellation across the different laboratories, seminars regarding instructions for samples drawing methods and use of quality index to measure the improvement obtained.

Key Words: risk errors, patient identification, cancellation

\section{INTERFERENCE SAMPLE AS A SOURCE OF PREANALYTICAL VARIABILITY: RESULTS OF CROATIAN PREANALYTICAL EXTERNAL QUALITY ASSESSMENT CROQALM PROGRAM}

\author{
N. Nikolac Gabaj ${ }^{3}$, I. Celap ${ }^{2}$, J. Lenicek Krleza ${ }^{1}$ \\ ${ }^{1}$ Department of laboratory diagnostics, Children's hospital; CROQALM, Croatian Society of Medical Biochemistry and Laboratory Medicine, \\ Zagreb, Croatia \\ ${ }^{2}$ University Department of Chemistry, Medical School University Hospital Sestre Milosrdnice; CROQALM, Croatian Society of Medical Biochemis- \\ try and Laboratory Medicine, Zagreb, Croatia \\ ${ }^{3}$ University Department of Chemistry, Medical School University Hospital Sestre Milosrdnice; Working group for preanalytical phase, Croatian \\ Society of Medical Biochemistry and Laboratory Medicine, Zagreb, Croatia
}

BACKGROUND-AIM: The aim of this study is to present results of distributing icteric sample within Croatian preanalytical External Quality Assessment (EQA) provider CROQALM. 
METHODS: All Croatian laboratories ( $\mathrm{N}=194)$ were invited to participate in free-of-charge preanalytical EQA during 2018. In March (Sample 1) and September (Sample 3) serum sample for clinical chemistry, while in June (Sample 2) citrate plasma for coagulation were distributed. Samples were prepared as follows: leftover serum/citrate plasma samples without any visible interference were collected, mixed, immediately frozen at $-20^{\circ} \mathrm{C}$ for 48 hours, thawed and filtered once using filter paper. Pools were spiked with powdered bilirubin (Sigma-Aldrich, Missouri, USA) in order to create icteric samples. Samples were aliquoted and sent out to laboratories within 48 hours. Participants were asked to evaluate the degree of interference. Responses were collected via service SurveyMonkey (California, USA). Variables were compared using Chi-square test in MedCalc (v12.7.2.0, Ostend, Belgium). $\mathrm{P}<0.05$ was considered statistically significant.

RESULTS: Majority of laboratories evaluate icteria visually (108/161, 67.1\%; 140/148, 94.6\%; 101/156, 64.7\% in cycles 1-3, respectively) with lower representation of automatic evaluation in coagulation (cycle 2; $\mathrm{P}<0.001$ ). When asked to visually evaluate the sample, 109/157, 69.4\%; 103/144, 71.5\%; 101/153, 66\% gave the preferred answer ("icteric sample"), no differences between cycles were observed ( $\mathrm{P}=0.450)$. Out of laboratories using automatic evaluation of icteria, significantly more expressed index quantitatively in cycle 3 vs. 1 . (78.8\% vs. 42.2\%; P<0.001). No laboratory provided data on automatic index in coagulation cycle. $2 / 3$ of laboratories express I index in $\mu \mathrm{mol} / \mathrm{L}$.

CONCLUSIONS: Large variability between Croatian laboratories was observed in management of icteric sample, with automatic evaluation being more implemented in clinical chemistry than in coagulation.

Key Words: preanalytical phase, external quality assessment, interference, icteria

\title{
THEORETICAL AND PRACTICAL KNOWLEDGE OF PRE-ANALYTICAL PHASE AND BLOOD SAMPLING AMONGST STUDENTS OF MEDICAL LABORATORY DIAGNOSTICS.
}

\author{
M. Čuljak $^{1}$ \\ ${ }^{1}$ Sestre Milosrdnice University Hospital Center, Zagreb, Croatia
}

BACKGROUND-AIM: The aim of this study was to examine and present theoretical and practical knowledge of the pre-analytical phase and blood sampling amongst students of medical laboratory diagnostics.

METHODS: The examination was conducted through a questionnaire on undergraduate and graduate students of the medical laboratory diagnostic study. Students responded by answering an anonymous questionnaire of six short laboratory questions about the pre-analytical phase and the degree of theoretical and practical knowledge of phlebotomy studies.

RESULTS: On the first question whether they are familiar with the pre-analytical phase of the laboratory process, 134 of 149 students answered yes (90\%) and 15 students answered no. To the question of evaluating pre-analytical phase, $27(18 \%)$ students answered that their knowledge was not well, 93 (62\%) students answered good, and 29 (19\%) of them said that they were excellent. 44 (30\%) students said that they had perfected venous and capillary blood sampling skills during the study program, and $105(70 \%)$ say that they did not. At 72 students (45.6\%), theoretical and practical knowledge of blood sampling was not well, $62(39.2 \%)$ was good, and 15 (15.2\%) of students was excellent.135 (91\%) students during the study program met with tube types and anticoagulants for blood sampling and 14 (9\%) did not. In the end, 144 (97\%) students considered that the pre-analytical phase should become an integral part of the curriculum, medical laboratory diagnostics, and 5 (3\%) students considered it not necessary.

CONCLUSIONS: According to the conducted research, students have learned average theoretical knowledge of pre-analytic, but less than half of them are not satisfied with the practical knowledge of blood sampling. The health professionals of the medical laboratory diagnostics study play an important role in the pre-analytical phase and the pre-analyst should become a separate unit of the curriculum, the medical laboratory diagnostic study.

Key Words: pre-analytic phase, students, blood sampling, medical laboratory diagnostics

\section{BLOOD SAMPLE COLLECTION PRACTICES IN THE EMERGENCY DEPARTMENT: ASSOCIATION WITH SAMPLE HEMOLYSIS}

\author{
I.A. Hashim ${ }^{2}$, A. Jones ${ }^{1}$, J. Klein ${ }^{1}$, S. Remonte ${ }^{1}$, D. Marchetto ${ }^{1}$, J. Cuthbert ${ }^{2}$ \\ ${ }^{1}$ Parkland Memorial Hospital, Dallas, Texas, USA \\ ${ }^{2}$ University of Texas Southwestern Medical Center, Dallas, Texas, USA
}

BACKGROUND-AIM: Hemolysis is the major reason for rendering samples unsuitable for biochemical analysis. Although hemolyzed samples are received into the laboratory from all locations, the majority originate at the Emergency Department (ED). In collaboration with Center for Disease Control (CDC) we examined blood collection practices to evaluate the effectiveness of evidence based recommended practices to reduce hemolysis rate in the ED

METHODS: Baseline hemolysis rates as well as blood samples collection practices in use at a medium-size ( 40 beds) and a large ( 250 bed) EDs were obtained. Practices were recorded via direct observation at both EDs as well as during staff interviews at the large ED following 
identification of hemolyzed samples. Using a data collection tool, the following sample collection variables were collected; use of straight needle or existing intravenous line, needle size, use of saline lock, use of syringe, use of J-loop and direct use of a vacutainer collection device. Practice variables as well as sample quality outcomes were analyzed

RESULTS: Baseline data were collected from a total of 218 patients admitted to both EDs. Hemolysis rate was $4.8 \%$ in the large ED compared with 1.3\% in the medium-size ED. Collections practices were different between the two EDs. The medium-size ED had 18 variations with two practices being predominant, whereas the larger ED had 24 variations with no predominant practice. Among hemolyzed samples $79 \%$ were collected using existing intravenous lines with 9 different combination of practices. Among the non-hemolyzed collections, only $6 \%$ used the existing intravenous lines to collect blood specimens

CONCLUSIONS: There was no standardized blood collection practices in the EDs with various options for needle size, and collection techniques. Although there was no particular practice responsible for hemolysis, the use of intravenous lines was commonly associated with hemolyzed samples.

Key Words: sample hemolysis, emergency department, pre-analytical, intravenous lines

\title{
A SURVEY OF PRACTICE IN THE MANAGEMENT OF HAEMOLYSIS, ICTERUS, AND LIPAEMIA IN BLOOD SPECIMENS IN THE UNITED KINGDOM AND REPUBLIC OF IRELAND
}

\author{
$\underline{\text { S. Costelloe }}{ }^{5}$, N. Goulding ${ }^{5}$, H. Mistry ${ }^{7}$, A. Stretton ${ }^{1}$, B. De La Salle ${ }^{8}$, S. Hepburn ${ }^{6}$, A. Thomas ${ }^{4}$, J. Atherton ${ }^{3}$, M. Cornes ${ }^{2}$ \\ ${ }^{1}$ Becton, Dickinson and Company, 1030 Eskdale Road, Wokingham, RG41 5TS, United Kingdom (UK) \\ ${ }^{2}$ Biochemistry Department, Worcestershire Acute Hospitals NHS Trust, UK \\ ${ }^{3}$ Blood Sciences Department, Liverpool Clinical Laboratories, Aintree University Hospital, UK \\ ${ }^{4}$ Cardiff and Vale University Health Board, UK \\ ${ }^{5}$ Department of Clinical Biochemistry, Cork University Hospital, Wilton, Cork, Republic of Ireland (ROI) \\ ${ }^{6} \mathrm{NE}$ Essex and Suffolk Pathology Services, UK \\ ${ }^{7}$ Serious Hazards of Transfusion Office, Manchester, UK \\ ${ }^{8}$ UK NEQAS Haematology, Watford, UK
}

BACKGROUND-AIM: Haemolysis (H), icterus (I) and lipaemia (L) are frequent interferants in laboratory medicine and result in incomplete reports, delayed patient management, repeated venesection, and increased cost. This survey reviews practice for the management of HIL in medical laboratories in the UK and ROI.

METHODS: A SurveyMonkey link was sent to members of the ACB, the Association of Clinical Biochemists in Ireland, and the Academy of Clinical Science and Laboratory Medicine (ROI).

RESULTS: Responses from 124 laboratories are broken down by country as follows: England (52\%); ROI (36\%); Scotland (7\%); Wales (2\%); and Northern Ireland (2\%). Most respondents were from public hospitals (90\%), with biochemistry services (90\%). Laboratories served primary care (91\%), inpatients (91\%), and outpatients (89\%), and monitored H (98\%), I (88\%), and L (96\%). Laboratories detected HIL using automated indices (51\%), visual inspection (8\%), or a combination approach (42\%). Manufacturer cut-offs for HIL are used by $83 \%$ of respondents. Cut-offs are applied to all general chemistries in 79\%, and all immunoassays in 50\% of laboratories. Where HIL cut-offs are breached, $64 \%$ withhold test results, and $96 \%$ report the interference to users. HIL were defined using numeric scales in $70 \%$, and ordinal scales in $26 \%$ of laboratories. HIL targets exist in 35\% of laboratories and 54\% have made attempts to reduce HIL. Internal Quality Control for HIL has never been performed in 62\% of laboratories and 18\% have never participated in External Quality Assurance. Laboratories agree manufacturers should: standardise HIL reporting (94\%); ensure comparability between platforms (94\%); and provide information on HIL cross-reactivity (99\%). Most respondents (99\%) showed interest in evidence-based, standardised, HIL cut-offs.

CONCLUSIONS: Although almost all respondents monitor HIL, a wide variation in practice may lead to differences in clinical care. Laboratories appear receptive to education and advice on HIL management from experts and industry.

Key Words: hemolysis, icterus, lipemia, serum indices

\section{ONE YEAR OF PRE AND POST-ANALYTICAL PERFORMANCE MONITORING FROM UK NEQAS}

\author{
B. De La Salle $^{2}$, F. Mackenzie ${ }^{1}$, R. Marrington ${ }^{1}$ \\ ${ }^{1}$ Birmingham Quality [UK NEQAS], University Hospitals Birmingham NHS Foundation Trust, Birmingham \\ ${ }^{2}$ UK NEQAS Haematology, West Hertfordshire Hospitals NHS Trust, Watford
}

BACKGROUND-AIM: There have been many initiatives to collect and collate benchmarking data on error rates in the Pre and Post Analytical aspects of Laboratory Medicine. We report here on the findings from the UK where there has been an established pre and post-analytical quality monitoring service (PREPQ) offered by UK NEQAS since 2017, following the Pilot phase from 2014 to 2016 . The service is directed by a multi-disciplinary steering group of advisors that includes international experts in the field of pre and post-analytical variables. 
METHODS: Participating Laboratories submit their error rates for a range of parameters either by Discipline, (e.g. Haematology) or by the whole service (e.g. Blood Sciences). The time periods are Calendar months. To avoid different counting practices Participants may classify their error rates by Request or Specimen.

RESULTS: The data is normalised into the currency of Sigma Scores, in addition to the raw data, since the size of Laboratories varies by over several orders of magnitude. Trend data is presented in a standard UK NEQAS style familiar to participants. Data has been returned from a maximum of 32 Laboratories for eleven quality indicators. In November 2018, for the key indicators of Sample time/temperature critical failures, the median Sigma Score is 3.85 but the spread is from 2.22 to 4.63. For Patient ID failures the data is a healthier 4.83, with a range of 3.3 to 5.64. Some Laboratories can be at opposite ends of the Sigma Score spectrum for different indicators.

CONCLUSIONS: Despite having detailed guidance, there remains confusion as to what data should be collected and how it could be collected in a standardised fashion. The challenge going forward is to review the quality indicators in use against the published literature, to work towards harmonisation of indicators within Europe and to have these built into the specifications for laboratory information management systems.

Key Words: pre-analytical, post-analytical, EQA, error, benchmarking

\title{
HOW TO CONTROL THE SERUM INDICES: THE EXTERNAL QUALITY ASSESSMENT SCHEME ORGANIZED BY THE SPANISH SOCIETY OF LABORATORY MEDICINE (SEQC-ML)
}

\author{
M.A. Llopis ${ }^{1}$, M. Ventura ${ }^{1}$, S. Bullich ${ }^{1}$, M.C. Gonzalez ${ }^{1}$, M. Cortés ${ }^{1}$, M. Ibarz ${ }^{1}$, A. Caballero ${ }^{1}$, J.M. Bauça ${ }^{1}$, I. García Del Pino ${ }^{1}$, C. Gómez ${ }^{1}$, \\ D. Martínez ${ }^{1}$, I. Marzana ${ }^{1}$, J.J. Puente ${ }^{1}$, M. Segovia ${ }^{1}$, R. Gómez-Rioja ${ }^{1}$ \\ ${ }^{1}$ Commission on Quality Assurance in the Extraanalytical Phase of the Spanish Society of Laboratory Medicine (SEQC-ML), Spain
}

BACKGROUND-AIM: The direct spectrophotometric measurement of free hemoglobin, jaundice or turbidity in laboratory samples (serum indices) is included in most clinical chemistry analyzers and it is the recommended way to systematically detect and quantify these three interferences in medical laboratory. One of the main drawbacks of these methods was the lack of internal and external quality controls. In this context the SEQCML decided to organize an EQA scheme to control the measurement of serum indices in medical laboratories.

METHODS: It is an annual program that includes 36 lyophilized control samples (12 for each index, 4 batches). The control material contains different known concentrations of hemoglobin, bilirubin or intralipid. The participating laboratories are grouped according to the analyzer they use and the way indices are reported: quantitative (numerical) or qualitative (interval).

RESULTS: After a pilot period, the program started in 2018 as a definitive program, currently having 165 registrations. Around $70 \%$ of laboratories report quantitative results. Global CV\% ranges from $5 \%$ to $11 \%$ for the hemolytic index, from $9 \%$ to $18 \%$ for the icterus index and from $12 \%$ to $20 \%$ for the lipemia index. The percentage of correct results (qualitative indices) ranges from $89 \%$ to $100 \%$ depending on the index. For the quantitative indices, the most used analyzers are Cobas ${ }^{\circledR}(67 \%)$, Architect ${ }^{\circledR}(15 \%)$ and Advia ${ }^{\circledR}(9 \%)$ and for the qualitative indices, the most used analyzers are Beckman Coulter AUß (44\%) and Dimension ${ }^{\circledR}(31 \%)$.

CONCLUSIONS: Data obtained show less dispersion for the hemolytic index than the icterus and lipemia indices (quantitative results) and similar percentage of correct results (qualitative results) for the three indices. There is a high percentage of laboratories reporting qualitative results. From our point of view, quantitative results should be reported in order to standardize the decisions on the results and to improve patient safety.

Key Words: serum indices, hemolysis, icterus, lipemia, external quality assessment scheme

\section{EVALUATION OF THE KNOWLEDGE AND PRACTICE REGARDING PREANALYTICAL PHASE AMONG MEDICAL STAFF IN THREE REGIONAL HOSPITALS IN TUNISIA}

\author{
$\underline{\text { H. Teyeb }}^{2}$, I. Houass ${ }^{4}$, M. Teyeb ${ }^{3}$, W. Douki ${ }^{1}$, L. Gaha ${ }^{4}$ \\ ${ }^{1}$ Biochemistry and Toxicology Laboratory, University Hospital Fattouma Bourguiba of Monastir, Monastir, Tunisia \\ ${ }^{2}$ Medical Biology Laboratory, Regional Hospital of Ben Guerdane, Ben Guerdane, Tunisia \\ ${ }^{3}$ Private Dialysis Center, Medenine, Tunisia \\ ${ }^{4}$ Research Laboratory “Vulnerability to Psychotic Disorders LR 05 ES 10”, Department of Psychiatry, Faculty of Medicine, University of Monastir, \\ Monastir, Tunisia
}

BACKGROUND-AIM: The preanalytical phase is the most vulnerable and challenging part of the total testing process of medical laboratory. Therefore, the quality of the preanalytical phase determines the reliability of laboratory results. The aim of our study was to assess the level of knowledge and practice on preanalytical phase among medical staff through a cross section survey.

METHODS: Survey was conceived as a questionnaire on preanalytical phase of laboratory testing, covering several aspects such as labeling, and causes of hemolysis. Our study included 95 participants (regardless age and work experience years) from three public regional hospitals in southern Tunisia (35 from Gabes Regional Hospital, 30 from Zarzis Regional Hospital and 30 from Ben Guerdane Regional Hospital). 
RESULTS: Survey results showed that most of prescription sheets didn't contain any clinical information. At the three regional hospitals, medical staff knowledge and awareness regarding sampling process remains insufficient which generates some practical deficiencies such as lack of proper patient's identification (name, age,..), collecting or handing specimens (52\%) and wrong use of syringes (67\%). Most of the participants (91.5\%) were aware that the quality of the preanalytical phase influences the reliability of the results and have been able to identify some causes of errors in laboratory testing process. The survey staff proposed an appropriate training to apply a pre-collection guideline for all medical staff (47.4\%) and adequate equipment improvement (25.3\%).

CONCLUSIONS: In conclusion, implementation of education programs concerning laboratory testing is needed to improve quality of preanalytical phase, specimen and subsequently patient care. Furthermore, the application of Laboratory Informatic System "LIS” (afterwards this study) and the total computerization of the data registration have emerged as valuable tools and potential strategies for decreasing preanalytical errors in medical laboratories.

Key Words: evaluation, medical staff, survey, preanalytical phase

\title{
SPECIMEN ACCEPTABILITY: AN EXAMPLE OF ONE LITHUANIAN LABORATORY EXPERIENCE
}

\author{
$\underline{\text { R. Stonys }}^{1}$, D. Vitkus ${ }^{2}$ \\ ${ }^{1}$ Centre of Laboratory Medicine, Vilnius University Hospital Santaros Klinikos, Vilnius, Lithuania \\ ${ }^{2}$ Institute of Biomedical Sciences of the Faculty of Medicine, Vilnius University, Vilnius, Lithuania
}

BACKGROUND-AIM: Specimen quality is a crucial factor that can affect test results and patient healthcare. The aim of this study was to determine the frequency and reasons of sample rejection in Laboratory of Biochemistry of the Centre of Laboratory Medicine of Vilnius University Hospital Santaros Klinikos.

METHODS: Specimen's rejection data were collected from Laboratory Information System (LIS) from January 1, 2017 to December 31, 2017. Specimen rejection reasons and frequency were evaluated monthly.

RESULTS: 389,446 specimens were received during 2017, and 3927 (1.01\%) were rejected as unsuitable for laboratory testing due to pre-analytical errors and therefore had to be recollected. Sigma metrics analysis showed that the performance was equal to 3.9 sigma ( () ). Main reasons for specimen rejection were: $50.83 \%$ of rejected specimens were haemolysed specimens, $7.38 \%$ - insufficient specimen volume, and $4.53 \%$ - clotted specimens for coagulation tests. Most rare rejection reasons were misidentification ( $0.01 \%$ of all received samples) and improper specimen transportation ( $0.005 \%$ of all received samples). $34.05 \%$ of rejected samples were classified on LIS as “other reasons".

CONCLUSIONS: The evaluation of specimen rejection reasons allowed to identify pre-analytical processes that have to be improved in order to provide best quality healthcare services for the patients. The research showed that main reason for specimen rejection was haemolysis, which means that additional phlebotomists training is required. High percentage of rejected specimens with reasons classified as "other reasons" requires to reconsider current grading to be made in more detailed way. Evaluation of additional training effectiveness will be done by measuring quality indicators on year 2018.

Key Words: pre-analytical phase, specimen rejection, quality indicators, patient satisfaction

\section{IMPACT OF A TRAINING ACTIVITY ON THE SAMPLE QUALITY}

\author{
$\underline{\text { S. Pico }}^{1}$, M. Bernal ${ }^{1}$, A. Munoz ${ }^{1}$, S. Carrasco ${ }^{1}$, A. Criado ${ }^{1}$, M. Ruestes ${ }^{1}$, M. Ibarz ${ }^{1}$ \\ ${ }^{1}$ Laboratory Medicine Department, University Hospital Arnau de Vilanova, IRBLleida, Lleida, Spain.
}

BACKGROUND-AIM: The haemolytic index (HI) has been postulated as a useful quality indicator of the extraction process and the main reason for rejection (RR) of the serum samples. Based on the EFLM-COLABIOCLI'S guideline "Recommendation for venous blood sampling", a training course destiny to our phlebotomy staff was carried out. The aim of this study was to evaluate the effectiveness of the course based on the change produced in the HI and RR indicators before and after the training.

METHODS: The extractor staff was trained and the change observed between the three weeks before and after the training was evaluated. The determination of the HI was performed in AU5800 of Beckman-Coulter analyzer. This index is based in a qualitative expression as whole values between 0 and 6 depending on the concentration of hemoglobin (Hb), with 1 (HI) corresponding to $<0.5 \mathrm{~g} / \mathrm{L}$ and 6 (HI6) $>5 \mathrm{~g} / \mathrm{L}$ of hemoglobin.

RESULTS: The total number of tubes before the training were 3204 and after 3418 . The percentage of hemolysed samples with $\mathrm{HI}>0.5 \mathrm{~g} / \mathrm{L}$ pretraining was $2.15 \%$ and post-training $1.61 \%$. Differences were more evident in HI1 and HI6: $1.19 \%$ vs. $0.82 \%$ and $0.22 \%$ vs. $0.03 \%$, respectively. The HI 1 was the cause of $16 \%$ of the rejections in the pre-training period and less than $1 \%$ at the post-training. For HI6 decreased from $20 \%$ to $4.5 \%$.

CONCLUSIONS: According to our results we conclude that the impact of the training was positive, both in terms of hemolytic index and percentage of rejection of samples. It is necessary to prolong the study time to confirm the results.

Key Words: indicators, haemolysis, training, preanalytical 


\title{
SAMPLING TRAINING BASED ON EFLM-COLABIOCLI’S RECOMMENDATION
}

\author{
M. Bernal ${ }^{1}$, S. Pico ${ }^{1}$, S. Carrasco ${ }^{1}$, A. Munoz ${ }^{1}$, A. Criado ${ }^{1}$, M. Ruestes ${ }^{1}$, C. Marzo ${ }^{1}$, M. Ibarz ${ }^{1}$ \\ ${ }^{1}$ Laboratory Medicine Department, University Hospital Arnau de Vilanova, IRBLleida, Lleida, Spain.
}

BACKGROUND-AIM: It's well known that most of the errors produced in the clinical laboratory occur in the extraanalytic phase, with the preanalytic phase concentrating the highest percentage of them. Therefore, it is very important to develop educational programs and to establish continuous educational courses for phlebotomy staff. In this context, it was offered a venous blood collection procedure training course for our phlebotomists.

METHODS: A sampling training course which included a theoretical and a practical training was given to the phlebotomy staff (20 hours). In accordance with the EFLM-COLABIOCLI'S recommendation for venous blood sampling, the course included the following issues: importance of the preanalytical phase, types of samples (plasma, serum, whole blood, capillary blood, biological fluids, etc.), tubes and containers, as well as the additives used (EDTA, sodium citrate, lithium heparin ...), order of draw, most common preanalytical errors (incorrect identification, haemolysis, coagulated or insufficient samples, contamination, etc.), consider appropriate solutions, error report models.

RESULTS: The contents of the training were evaluated at the end of the course through a knowledge test and an observational audit. The results were given as Approved and Not Approved. $100 \%$ of the staff passed the course and they were awarded a certificate of attendance and achievement.

CONCLUSIONS: The course was very well accepted by the professionals and the results proved its effectiveness in the short term. The impact of the action will be assessed according to the pre and post-course quality indicators.

Key Words: training, preanalytical, phlebotomy, venous blood sampling

\section{INFLUENCE OF A FASTER CENTRIFUGATION ON ROUTINE SERUM AND PLASMA SAMPLES IN CLINICAL CHEMISTRY AND HEMOSTASEOLOGY - MEASURED VALUES AND TURN-AROUND-TIME}

\author{
$\underline{\text { P. Neyer }}^{1}$, M. Hanke ${ }^{1}$, A. Huber ${ }^{1}$, A. Hammerer-Lercher ${ }^{1}$, A. Méndez ${ }^{1}$, L. Bernasconi ${ }^{1}$ \\ ${ }^{1}$ Institute of Laboratory Medicine, Kantonsspital Aarau AG, Switzerland
}

BACKGROUND-AIM: Centrifugation settings have a major impact on serum and plasma quality and TAT. Faster regimens elevate the risk of in vitro hemolysis or imperfect separation of the cell compartment. We investigated the effects of reduced centrifugation time and stronger centrifugal force on the analytical quality and TATs.

METHODS: Consecutive samples sent to our lab with supplementary plasma/serum tubes were included in the validation study. Samples were simultaneously centrifuged at a relative centrifugal force (RCF) of $1900 \mathrm{x} \mathrm{g}$ for 10 minutes and of $3000 \mathrm{x}$ g for 5 min. Selected chemistry, immunological and hematological parameters were studied in serum/plasma (lithium heparinate and citrate) with gel separator. Results were statistically evaluated based on parametric tests and difference plots. Turn-around time was compared for two periods of 30 days, before and after implementation of the new centrifuging settings.

RESULTS: The observed mean biases to the original values were below $10 \%$ for all parameters studied. Turn-around time from registration of the sample until validation by a laboratory technician could be reduced by a mean $4.5 \mathrm{~min}$ over all the evaluated parameters.

CONCLUSIONS: The critical parameters associated with in vitro hemolysis like lactate dehydrogenase (LDH), potassium (K), magnesium (Mg) and hemolysis index (Siemens) did not show a significant rise and TATs were shortened without draw-backs in quality of the results.

Key Words: centrigugation, hemolysis, turn-around time

\section{ELECTRONIC TEST REQUEST SYSTEM CHANGES THE DIRECTION OF PREANALYTICAL ERRORS}

\author{
B. Willman $^{1}$, C. Selenius ${ }^{1}$ \\ ${ }^{1}$ Department of Medical Biosciences, Clinical Chemistry, Umeå University, Sweden
}

BACKGROUND-AIM: Within the County council of Västerbotten, Northern Sweden, 7 million analysis are performed at the Laboratory of Clinical Chemistry with about 4500 samples (blood, urine, faces etc.) passing the laboratory daily. With every sample taken, there is an imminent risk of errors endangering patient safety. The highest error frequency is found in the preanalytical phase, before the sample arrive to the laboratory. In this study, we aim to elucidate the preanalytical error frequency on incoming samples to Clinical Chemistry with focus on consequences of using an electronic test request system.

METHODS: During three days in October 2018, personnel at clinical chemistry laboratories within the Count council of Västerbotten noted preanalytical errors on every incoming sample. This was done with a chart accessible for all staff positioned at arrival point of the laboratory. 
Moreover, statistics of rejected test results recorded in the laboratory data system during the same time interval was also apprehended. Data are presented with descriptive statistics.

RESULTS: In total 13439 samples were registered at the Clinical Chemistry Laboratories. Of these 1,53\% $(n=206)$ had preanalytical errors demanding action from laboratory personnel for test results to be released to client, whereas 1,47\% ( $\mathrm{n}=198)$ of all samples resulted in rejected test result. Errors associated with the "sampling procedure and transport", had the highest error frequency of 1,47\% (n = 198). Runner up with $1,23 \%(\mathrm{n}=165)$ was "test request errors" with "test request not sent" accounting for $79 \%(\mathrm{n}=131)$ of those errors.

CONCLUSIONS: With electronical test request system, the most time-consuming preanalytical error to be handled by laboratory personnel was identified as "test request not sent". This led us to conclude that technical solutions are helpful, however organizational changes are prompted in order to counteract this new direction of preanalytical errors.

Key Words: Preanalytical phase, clinical chemistry, electronic test request

\title{
RISK MANAGEMENT IN THE PREANALYTICAL PHASE OF A NEWBORN SCREENING PROGRAM
}

\author{
$\underline{\text { A. Soler }}^{3}$, L. Guiñón ${ }^{3}$, R. Lopez ${ }^{1}$, J.L. Marín ${ }^{1}$, J. García ${ }^{1}$, W. Jiménez ${ }^{1}$, Á. Mira ${ }^{2}$, L. Alvarez ${ }^{3}$ \\ ${ }^{1}$ Biochemistry and Molecular Genetics, Biomedical Diagnostic Center, Hospital Clinic, Barcelona, Spain \\ ${ }^{2}$ Direction, Biomedical Diagnostic Center, Hospital Clinic, Barcelona, Spain \\ ${ }^{3}$ Quality Unit, Biomedical Diagnostic Center, Hospital Clinic, Barcelona, Spain
}

BACKGROUND-AIM: Most of the errors that can affect patient safety occur in the extra-analytical phase. In the newborn screening programmes many actors from different types of clinical institutions are involved, such as doctors, midwives, messengers. This is the reason why many errors related to the pre-preanalytical phase are out of the laboratory control. Knowing the potential risks of this phase, together with those of the preanalytical phase, may help laboratories to design improvement actions. The aim of this study is to demonstrate the usefulness of risk management in the context of a newborn screening program.

METHODS: Pre-analytical risks of the newborn screening program were evaluated through a Failure Mode and Effects Analysis (FMEA). This study comprise five steps: 1-identification and mapping of the process steps; 2-identification of failure modes; 3 - evaluation and calculation of the Risk Priority Number (RPN); 4-identification of improvement actions and 5-follow-up and re-evaluation of the identified failures modes. RESULTS: Fourteen pre-preanalytical and six preanalytical failures modes were identified. Among the failures belonging to the pre-preanalytical phase, those with the highest RPN values were "sample extraction is not performed" and "erroneous essential data is registered in the neonatal file". Among the failures belonging to the preanalytical phase those with the highest RPN values were "errors during the sample transfer in to the plate" and "errors during the registration of the neonatal data in the SIL". After the implementation of the improvement actions, lowest RPN values were found during the two year follow-up period.

CONCLUSIONS: The introduction of risk management in the newborn screening program was useful to reduce errors of the pre-preanalytical and preanalytical phases. However, improvement actions must be continuously applied to ensure patient safety.

Key Words: risk management, errors, FMEA

\section{REVIEW OF TOTAL TURNAROUND TIME (TTAT) OF PATHOLOGY RESULTS FROM A BUSY EMERGENCY DEPARTMENT}

$\underline{\text { A. Leonard }}^{1}$, G. Boran ${ }^{1}$, D. Conway ${ }^{4}$, S. Hartigan ${ }^{3}$, F. O Donoghue ${ }^{2}$, N. Kyle ${ }^{3}$, B. Malone ${ }^{3}$, R. Cannon ${ }^{2}$, G. Nfila ${ }^{4}$, F. Brady ${ }^{4}$, H. Baker ${ }^{4}$, U. Fox ${ }^{4}$ ${ }^{1}$ Clinical Biochemistry unit, Trinity College Dublin, Ireland

${ }^{2}$ Emergency department, Tallaght University Hospital, Dublin, Ireland

${ }^{3}$ Emergency Department, Tallaght University Hospital, Dublin, Ireland

${ }^{4}$ Laboratory Medicine Department, Tallaght University Hospita, Dublin, Ireland

BACKGROUND-AIM: A significant amount of medical decisions are based on results from the laboratory analysis of specimens. The total turnaround time (TTAT) from sample taken to result available therefore may have a significant impact on patient care. The aim of this project was to improve and streamline the efficiency of taking and sending bloods form a busy emergency department (ED) to the laboratory for the benefit of staff and patients.

METHODS: A laboratory and ED continual improvement working group was established. The SMART aim statement for the project was developed. An audit template was developed and piloted. Following minor adjustments an audit was completed over a ten day period across ED and the laboratory. The audit was completed for the hours of 8am to 8pm Monday to Friday. In addition an 'out of hours' (8pm to 8am) audit was completed in the clinical chemistry department using the label print time as an indication of sampling time.

RESULTS: During 8am to 8pm the average TTAT was 1 hour (hr) and 13 minutes (min) with a standard deviation (SD) of 33 mins from sample taken to result available. The longest TTAT was $5 \mathrm{hrs}$ and $56 \mathrm{mins}$ with numerous samples taking over 2 - 3 hrs. The average time from sample taken to leave ED was 33 mins (SD 22 mins) with an average time of sample transport from ED to the laboratory of 12 mins (SD 24 mins) with one sample taking over $4 \mathrm{hrs}$ to reach the laboratory. TAT in the laboratory department was more consistent with average TAT of 33 mins and 
a SD of 10 mins. On review of the averages for each time period measured, 55\% of the TTAT can be accounted for by traveling through ED and transport to the laboratory and only $45 \%$ of TTAT can be accounted for by time spent in the Laboratory.

CONCLUSIONS: The audit results identified areas that had the potential for significant improvement. To date the team has completed a number of initiatives to improve the transport time for samples from ED to the Laboratory.

Key Words: Total Turnaround Time (TTAT), emergency department, continual improvement

\title{
NATIONAL SURVEY ON PREANALYTICAL ERROR MONITORING IN IRISH CLINICAL LABORATORIES
}

\author{
$\underline{\text { A. Leonard }}^{1}$, A. Kane ${ }^{2}$, G. Boran ${ }^{1}$ \\ ${ }^{1}$ Clinical Biochemistry unit, Trinity College Dublin, Ireland \\ ${ }^{2}$ Irish External Quality Assessment Scheme (IEQAS), Dublin, Ireland
}

BACKGROUND-AIM: Specimen labelling and patient identification is a significant contributor to the rate of error in the preanalytical phase. The aim of this project, was to describe the prevalence and nature of preanalytical quality monitoring practices of patient identification and sample labelling errors in Ireland.

METHODS: A survey was developed at the clinical biochemistry unit, Trinity College Dublin in conjunction with the Irish External Quality Assessment Scheme (IEQAS). The survey was subdivided into seven sections to gather information on specimen labelling and patient identification. The sections included: Labelling requirements, information availability, rejection criteria, error monitoring, error reporting and interest in participation in an external quality assurance scheme. The survey consisted of 39 questions in total. The survey was sent to 64 laboratory/ quality managers at 55 laboratories in the Republic of Ireland

RESULTS: A total of 39 responses were received from a range of departments and disciplines (61\%). Hospitals reported varying practices and requirements for labelling specimens and all accepted hand written request forms. $100 \%$ of respondents had defined rejection criteria both for specimen labelling and request form completion. Unsurprisingly the rejection criteria differs across disciplines in laboratories. Almost all respondents provided information to clinical staff on labelling requirements but just over half provided training on the same. A large percentage (74\%) of laboratories monitor the rate of specimen labelling errors, however only $46 \%$ have defined target limits for acceptable rates of error. CONCLUSIONS: The survey observes a wide variation in collection, recording and monitoring of errors. Data also indicates there is a lot of interest in improving preanalytical monitoring and data collection. Finally $89 \%$ of respondents were interested in participating in a national external quality assessment (EQA) scheme

Key Words: survey, preanalytic, error, laboratory

\section{IFCC AUTOMATED PRE-ANALYTICAL QUALITY INDICATORS - YES WE CAN!}

\author{
A.C. Silva ${ }^{1}$, M.A. Almeida ${ }^{1}$ \\ ${ }^{1}$ Centro Hospitalar de Entre Douro e Vouga - Serviço de Patologia Clínica, Santa Maria de Feira, Portugal
}

BACKGROUND-AIM: Since most laboratory errors (about 70\%) happen during preanalytical (PA) phase, standardization of all steps is quite crucial, as is the implementation of some monitorization tools to provide information on quality indicators (QI), follow all the steps in the process and assess the impact of improvement. This paper aims to explore the adoption of the QI proposal presented by IFCC Working Group in "Laboratory Errors and Patient Safety" (WG-LEPS) to the Indexor solution implemented in Centro Hospitalar Entre Douro e Vouga (CHEDV), as a data obtaining method and procedure standardization.

METHODS: All Mini-Indexor ${ }^{\circledR}$ are connected to each other through a network and track samples since collection until they are trashed. All records are sent to the lab's informatic system and, thus, it can obtain information on the actual collection date and time, as well as an automatic registration of the arrival of the samples, avoiding extra steps on this process. This information is also helping on monitorization and standardization of the preanalytical process, which allows to define a complete set of QI resulting from the use of the Indexor ${ }^{\circledR}$ solution. Selected QI were: Pre-MisR, Pre-MisS, Pre-NotRec, Pre-ExcTIm, Pre-UrgIdle, Pre-TAT, C_in-TAT.

RESULTS: For three months, 22.667 samples from a total of 1.182 transport racks were monitored. This way, the laboratory was able to define the initial quality of the PA processes by using QI and defining the purposes to achieve. It also helped justifying internal processes with procedural errors.

CONCLUSIONS: The implementation of QI in PA phase proved to be critical when it comes to monitorization and improvement. The Indexor ${ }^{\circledR}$ system allows for standardization of data obtaining methods without the need of any additional steps (time-consuming procedures). It is now possible to reduce errors and implement quality improvements based on evidence, thus increasing efficiency.

Key Words: preanalytics, quality indicators, standardization, Indexor®, time-consuming procedures 


\title{
COMPLIANCE OF THE PNAEQ PREANALYTICAL SCHEMES WITH THE ISO 15189:2012
}

\author{
$\underline{\text { A. Cardoso }}^{1}$, C. Ventura ${ }^{1}$, S. Viegas ${ }^{1}$, H. Correia ${ }^{1}$, A. Faria ${ }^{1}$ \\ ${ }^{1}$ Instituto Nacional de Saúde Dr. Ricardo Jorge, Departamento de Epidemiologia, Unidade de Avaliação Externa da Qualidade
}

BACKGROUND-AIM: The aim of participation in EQAs schemes on Preanalytical Phase is to sensitize clinical laboratories for the implement tools to evaluate preanalytical phase. PNAEQ and PNAEQ/Labquality provides schemes that allow the complying with the management and technical requirements of ISO 15189:2012.

METHODS: In 2018 were distributed 6 different surveys. For Indicators, it was sent a worksheet for monitoring 20 indicators for trimester. For Audits, it was sent a checklist for auditing yearly the blood sampling to identify errors, based on observation of 5 draws/technician. For Mystery Client, it was made anonymous phone calls based on a script, repeated in different days/hours, twice a year. For Simulated Cases, were available online cases to errors detection, once/twice a year. For Sample Quality, it was sent two samples for semester to serum interferers determination. For Training Actions, was organized one workshop with case studies presentation.

RESULTS: The follow requirements of ISO 15189:2012 are reflected in the available tools on PNAEQ Preanalytical schemes. For Indicators: 4.14.7. Quality indicators; 5.4.2 j) Information for patients and users; 5.4.3 Request form information; 5.4.4 Primary sample collection \& handling; 5.4.5 Sample transportation; 5.4.6 Sample reception. For Audits: 4.14.6. Risk Management; 5.1 Personnel; 5.4.4 Primary sample collection \& handling. For Mystery Client: 5.1 Personnel; 5.4.2 Information for patients and users. For Simulated Cases: 5.4 Pre-examination procedures. For Sample Quality: 5.4.6 Sample reception. For Training Actions: 5.1 Personnel.

CONCLUSIONS: PNAEQ provides tools that, when properly used in laboratory routine, allow to monitor preanalytical phase, evidence good laboratory practices and comply with regulatory requirements, ensuring correct attendance, collection and handling of the patient and the sample, leading to the correct results.

Key Words: preanalytical phase, regulatory requirements, indicators, audits, mystery client

\section{WITH EYES WIDE SHUT - WHAT AREN'T WE SEEING? MANAGING THE CLINICAL EXPECTATION TIME AT THE PREANALYTICAL PHASE}

\author{
J.G. Frade ${ }^{1}$, E. Costa $^{1}$, L. Araújo ${ }^{1}$, F. Rodrigues ${ }^{1}$ \\ ${ }^{1}$ Clinical Pathology Department, Centro Hospitalar e Universitário de Coimbra, Portugal
}

BACKGROUND-AIM: The preanalytical phase is critical in the daily routine of Clinical Laboratories, frequently occurring outside its influence. Processing begins upon sample arrival and, with it, Turn-Around-Time (TAT) determination; however, for Clinicians the waiting time begins with the request. The Sample-Request-To-Sample-Arrival time (SRSA) means that the Clinician-Expectation-Time (CET, proposed as the time period between request completion in the ER and result's validation) is often significantly higher than TAT. Because of this, the latter is often considered to be miscalculated whenever reported, potentially undermining how the Clinicians perceive Clinical Laboratories' response time. Therefore, we set to evaluate these time periods in the emergency room (ER) of a Portuguese Tertiary Hospital.

METHODS: The SRSA and TAT were determined once monthly in four trimesters, allowing for a one-year time monitoring of ER activity. Data from 39614 samples was collected from the laboratory information system, where all chronological details of the request and results are automatically recorded by the Clinician and Laboratory Staff. CET was calculated by the sum of SRSA and TAT.

RESULTS: On average, mean SRSA time was $44.7 \mathrm{~min}$ and the TAT $53.4 \mathrm{~min}$, with differences depending on time of year. The CET was $98,2 \mathrm{~min}$, roughly matching the Clinicians' perception of TAT (1-2 hours, as obtained after a short survey) and almost doubling Laboratory’s TAT. Blood tube used in the ER was lithium/heparin (eliminating the need for clot formation), theoretically allowing sample processing immediately after phlebotomy.

CONCLUSIONS: Unaware to Laboratory Staff, SRSA depends only on Clinicians' requests and phlebotomy. As CET is almost twice as real TAT, Clinical Laboratories remain with eyes wide shut in setting solutions that prevent complaints and degradation of Laboratory-ER daily workflow, starting from sample traceability improvement, collection and transport.

Key Words: turn around time, clinician expectation, sample traceability

\section{MANAGEMENT OF THE PRE-ANALYTICAL ERRORS IN THE HEMOSTASIS PROCESS}

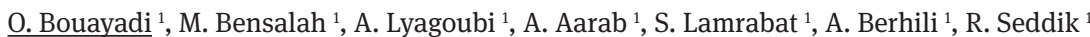

${ }^{1} 1$-Laboratory of Hematology, Mohammed VI UniversityHospital 2-Faculty of Medicine and Pharmacy (University Mohammed the first), Oujda, Morocco

BACKGROUND-AIM: Pre-analytical steps, the major source of mistakes in laboratory diagnostics, arise during patient preparation, sample collection, sample transportation, sample preparation, and sample storage. Among the indicators set up within the laboratory, those 
concerning Pre-analytical errors are particularly interesting. The objective of our work is to analyze and evaluate the pre-analytical erros in hemostasis phase, to identify the failures in order to improve and ensure the quality of this process.

METHODS: This is a cross-sectional study of pre-analytical errors found within the haematology laboratory of Mohammed VI University Hospital, over a period of 3 months.

RESULTS: During the study period 4200 samples for a hemostasis assessment were received at the laboratory. We studied separately the errors related to the specimens, those related to the identification of sampling tubes, those attached to the prescription of the hemostasis assessment and those linked to the conditions of transportation. In fact, of the 4200 samples, 462 specimens were not-conform, which corresponds to a rate of $11 \%$. Errors related to the disrespect of the ratio blood / anticoagulant is the most represented (78\%) followed by coagulated blood (14\%). Furthermore errors of prescriptions were in the order of $91 \%$. Errors related to the identification of the specimen (9\%) and those attached to the sample transportation (4\%). These pre-analytical erros come mainly from the emergency department (18\%), intensive care unit $(13 \%)$ and pediatrics (10\%).

CONCLUSIONS: Critical analysis of the different sources of errors in the pre-analytical phase allowed us to target the areas for improvement and to set up corrective actions based on the requirements of the standard.

Key Words: haemostasis, pre-analytical phase, errors

\title{
TRACKING PREANALYTICAL ERRORS IN CLINICAL LABORATORY
}

\author{
$\underline{\text { K. Krubnerová }}^{1}$, B. Srpová ${ }^{1}$, L. Pospíšilová ${ }^{1}$ \\ ${ }^{1}$ Department of Clinical Biochemistry, Military University Hospital Prague, Prague, Czech Republic
}

BACKGROUND-AIM: Laboratory testing is a complex process which consists of several phases. The most critical phase with the highest amount of errors is the preanalytical phase. It happens because the vast majority of preanalytical phase procedures is performed outside of the laboratory without proper laboratory professionals' control. These mistakes might have a significant effect on the sample and thus the test result could be negatively affected. Aim of this study is to present types of preanalytical errors in our laboratory, how often do they occur and how we handle them.

METHODS: Data were collected from October 2017 to September 2018. Every sample error is monitored by our laboratory information system, hemolytic index (HI) is set as $\mathrm{HI}>100$ (hemoglobin $>100 \mathrm{mg} / \mathrm{dL}$ ). We identified 10 types of preanalytical errors along with their occur frequencies. Furthermore, departments with the highest error rates were detected.

RESULTS: There were 165406 requests received by our laboratory during evaluated period, of which 2970 (1,8\%) were errors. Hemolysis errors have showed as the most frequent. They occured in approximately $72,3 \%$ of all monitored cases (there were overall 2147 hemolysis errors). Emergency department was detected as the most problematic. There were 1173 hemolysis errors monitored which makes about $8,1 \%$ of overall 14457 requests recieved only by this department.

CONCLUSIONS: Hemolysis was identified as the main cause of preanalytical errors in our laboratory. Furthermore, results showed that hemolysis is significantly associated with the emergency department.Preanalytical phase is one of the most important and the most crucial part of the whole laboratory testing process with signifficant effect on the test result hence we are trying to keep medical staff being educated and to improve quality of work with the purpose of decreasing mistakes, that are being made in preanalytical phase. For the purpose of ensuring patient safety, high quality standards are necessary to be kept all the time.

Key Words: preanalytical phase, preanalytical errors, hemolysis

\section{IMPLEMENTING A DYNAMIC DASHBOARD FOR PREANALYTICAL PHASE - RAISING AWARENESS LEADS TO ERROR REDUCTION}

\author{
I. Cachapuz Guerra $^{1}$, V. Lopes Dos Santos ${ }^{1}$ \\ ${ }^{1}$ CoreLab, Clinical Pathology Service, Pedro Hispano Hospital, Local Health Unit of Matosinhos, Matosinhos, Portugal
}

BACKGROUND-AIM: The Pre-analytical (PA) stage accounts for over 70\% of the errors identified in the Total Testing Process. Most of these errors follow from a limited awareness in the Core Lab (CL) to what happens in this stage, which is best explained by the lack of proper data to support its control. With the use of Indexor ${ }^{\circledR}$, a technology for sample traceability, the Pathology Service from the ULSM looked for building a Key Peformance Indicators (KPIs) real-time monitoring tool of the PA stage. The aim is that having visible data about this stage on the CL may allow for an increased awareness.

METHODS: Sample Full traceability data through Indexor ${ }^{\circledR}$ System at the CL for sample reception and Mini-Indexors ${ }^{\circledR}$ at the Collection Stations (CS), a set of KPIs calculated in real-time and displayed in a Dashboard visible to all the CL staff.

RESULTS: A Dashboard with KPIs was implemented on June 2018, presenting, for the current workday: i) number of patients, ii) number of tubes and samples collected, discriminated by CS differentiatiing between collected and received, iii) number of transports, iv) number of errors identified (missing samples, non-identified samples, temperature and time of transport exceeded, number of impacts), v) average idle 
time in minutes after check-in, vi) general Turn Around Time since collection, vii) hourly workload, viii) number of samples by work status (collected, in work, completed testing). Implementation led to raised awareness of CL staff towards PA stage challenges that before were inaccessible and are now presented in a objective, simple and structured graphical view, thanks to the availability of data and automatic calculation of KPIs without adding any steps both to the PA and the CL stages. PA errors decreased in $43 \%$ right in the first month.

CONCLUSIONS: The displayed KPIs allowed for the CL staff to act more proactively in the PA stage cooperating with the PA stage staff for better performance, proving that raised awareness will lead to error reduction.

Key Words: preanalytical phase, performance indicators, quality, continuouos improvement

\title{
TIME PERFORMANCE REPORTS FROM AN EMERGENCY SERVICE
}

\author{
M. Pereira ${ }^{1}$, I. Cachapuz ${ }^{1}$ \\ ${ }^{1}$ Unidade Local de Saúde de Matosinhos, Matosinhos, Portugal
}

BACKGROUND-AIM: Turnaround time (TAT) is one of the most noticeable signs of laboratory service and is often used as a key indicator of laboratory performance. Preanalytic phase is an important component of laboratory quality. A 90\% completion time (from sample registration to result reporting) of less than 60 minutes for common laboratory tests is suggested as an initial goal for an acceptable TAT.

METHODS: This study aimed to evaluate the TAT of the following analytes: potassium, C-reactive protein (CRP), troponin I, pancreatic amylase and B-type natriuretic peptide (BNP) from emergency service patients during 1 month (October 2017), between 8.00 a.m. and 8.00 p.m.

RESULTS: In total, the average t of TAT response was of 00:59:32. Potassium had the lower average TAT (00:34:45) whereas BNP had the highest average TAT (1:35:24). Troponin I had faster "Prescription to collecting sample" $t$ (0:03:09) but had not the best $t$ concerning "Collecting sample to medical validation" (1:07:22), which is held by potassium (0:22:37). Creatinine had the worst "Prescription to collecting sample" $t$ (0:23:42). The maximum "Prescription to collecting sample" $t$ was observed for pancreatic amylase (3:14:03), whereas the minimum was observed for Creatinine and Potassium (both 0:00:50). The maximum "Collecting sample to medical validation" $t$ was observed for Troponin I (4:17:38), whereas the minimum was observed for Potassium (0:18:59). The maximum TAT was observed for Troponin I (6:52:08), whereas the minimum was observed for Creatinine (0:25:18).

CONCLUSIONS: In order to improve clinical and laboratory interface, it is very important to have quick answers, standardized practices and good communication of results

Key Words: TAT, preanalytical phase, quality, emergency service

\section{A QUALITY IMPROVEMENT APPROACH TO REDUCE LABORATORY SAMPLE REJECTIONS DUE TO PRE-ANALYTICAL ERRORS IN A PAEDIATRIC HOSPITAL}

\author{
W. Musabaike ${ }^{1}$, C. Morris ${ }^{1}$, Y.C. Wu ${ }^{1}$, T. Rockenbach ${ }^{1}$, M. Nakrani ${ }^{1}$, S. Heales ${ }^{1}$ \\ ${ }^{1}$ Great Ormond Street Hospital for Children, London, UK
}

BACKGROUND-AIM: Improving the pre-analytical process is complex because of the involvement of several staff groups and stakeholders. Laboratories usually do not have direct control of the process. At Great Ormond Street Hospital, it was acknowledged that there was under reporting of pre-analytical errors (PAE) with no coordinated approach to correct the errors. An organisation wide quality improvement approach was used to reduce the number of PAE. We describe this approach and provide 2 case examples.

METHODS: A member of staff was recruited to specifically develop pre-analytical practice, policy and procedures. Stakeholder mapping identified key internal and external stakeholders who were engaged to surface priority pre-analytical issues. Sponsorship of the organisation's executive was sought and obtained. A driver diagram was used to identify primary and secondary drivers influencing the occurrence of pre-analytical errors. These were 1) sample collection resources; 2) transportation; 3) policies and 4) training. Data collection methods were standardised and dashboards were placed on the intranet.

RESULTS: Case 1: Data showed that 7.3\% of nasopharyngeal aspirates (NPAs) had leaked in transit to the laboratory due to transportation in the chute system. A communication was issued to prohibit use of the chute for NPAs. This resulted in a $65 \%$ drop in the number of leaking samples. Case 2: A campaign was initiated to reduce blood culture transport time from ward to laboratory. Average transport was significantly reduced from before ( $\mathrm{n}=1277,318$ minutes) to after ( $\mathrm{n}=927,268$ minutes) the campaign, ( $\mathrm{p}$ value $<0.001$ ).

CONCLUSIONS: The approach we have adopted has yielded buy-in from key stakeholders. We have improved local data collection. Some of the interventions we have implemented have shown demonstrable quality improvement while others are in progress. We have significantly reduced the number of leaking NPAs. Average blood culture transport time was reduced following a campaign.

Key Words: approach, improvement, paediatric 


\title{
ANALYSIS OF ERRORS IN THE CHEMICAL PATHOLOGICAL ANALYSES OF BLOOD SAMPLES IN LABORATORIES FOUND IN OWERRI IMO STATE.
}

\author{
$\underline{\text { I. Olewuike }}^{1}$ \\ ${ }^{1}$ Department of Medical Laboratory Science of Imo State University Owerri imo state Nigeria
}

BACKGROUND-AIM: The aim of the study is to identify the root and remote causes of analytical errors in laboratory analysis. METHODS: Methods adopted in this study are random sampling of reported cases of errors of analysis of blood samples for chemical Pathological analyses, and testing for any significant relationship between the the errors and the laboratory some listed environmental and mechanical factors.

RESULTS: The result of this investigation showed significant relationship between observed chemical Pathological analyses of blood samples and some environmental and mechanical factors such as, vibrations caused by heavy mechines within the laboratory building, the materials that are used in sampling containers and some environmental factors included secondary infections of the samples and varying temperature of the samples.

CONCLUSIONS: The study thus concluded that vibration causing machines like power generating sets, pokers etc should not be located within the laboratory building. There should also be temperature regulation of the storage facilities and overall analysis room.

Key Words: preanayltical phase

\section{LEAN SIX SIGMA IN CLINICAL LABORATORY: PRE-ANALYTICAL PROCESS MANAGEMENT - FOCUS ON REPEATED HARVESTING AND RESPONSE TIME OF RESULTS}

\author{
$\underline{\text { R. Ribeiro }}^{1}$, M.J. Sousa ${ }^{1}$, M. Albuquerque ${ }^{1}$, J.G. Sousa ${ }^{1}$, G. Sousa ${ }^{1}$ \\ ${ }^{1}$ Centro de Medicina Laboratorial Dr. Germano de Sousa, Lisboa, Portugal
}

BACKGROUND-AIM: The philosophy of Six Sigma quality can be seen as a strategy that achieves perfection in products and processes. This holistic view was a new tool for the Clinical Laboratory, which in the context of business competitiveness concerns the relentless pursuit of perfect products by establishing an organizational advantage in various aspects. From the standpoint of the user, there are two perceived characteristics: accurate and unambiguous results, and the time of delivery. The Six Sigma project developed in Centro Medicina Laboratorial Germano de Sousa can bring great benefits to the pre-analytical and analytical process. Using the impact of using Lean Six Sigma methodologies in the management of the process, focusing on repeated harvesting and response time, maintaining the quality of analytical results. METHODS: Analysis of repetitions per parameter for each equipment, studies of reproducibility and repeatability.The reduction of repeated harvest and repetitions performed per equipment improvement was measured in Sigma metric. The statistically tests performed in Minitab software.

RESULTS: According to the studies of reproducibility and repeatability, along with sigma metric as an index of performance we want to standardize the analytical repetitions performed per parameter to confirm the results, thus reducing repeated harvesting, the process time and achieved higher efficiency, additional expense of reagents. In this project the TAT improvement was $36 \%$ reduction time and repetitions performed per equipment improvement was Sigma 3.50 to 4.00. This contribute to achieved a reduction of repeated harvest in $12.38 \%$. CONCLUSIONS: The use of a Six Sigma strategy in the clinical laboratory, not just being used as a metric for performance analysis, but in the context of case management provides a standardization of a complex and multidisciplinary, aligns the goals of quality and costs, focusing on customers and the health system's financial organization.

Key Words: Lean/Six Sigma, repente harvesting, response time

\section{QUALITY INDICATORS TO DETECT PRE-ANALYTICAL ERRORS: EXPERIENCE IN CENTRO MEDICINA LABORATORIAL GERMANO DE SOUSA}

\author{
M.J. Sousa ${ }^{1}$, R. Ribeiro ${ }^{1}$, M. Albuquerque ${ }^{1}$, J.G. Sousa ${ }^{1}$, G. Sousa ${ }^{1}$ \\ ${ }^{1}$ Centro de Medicina Laboratorial Dr. Germano de Sousa, Lisboa, Portugal
}

BACKGROUND-AIM: The constant technological development and scientific advances in the new equipment and tests, the type of errors and their frequency in the Clinical Laboratory have been changing. Nevertheless, the Preanalytic Phase absorbs the largest number of errors (46\% to $68 \%$ ). The main reason is the difficulty in controlling the pre-analytical variables, such as harvesting, handling, transporting and sample preparation. The use of quality indicators (QI) has been valued in the management of clinical laboratories to optimize the qualification and quantification of failures in the laboratory processes, as well as to assist in the implementation of corrective and preventive measures and 
to indicate the effectiveness of the actions taken. In Centro Medicina Laboratorial Germano de Sousa we use several QI in the pre-analytical phase. The aim of this study is to evaluate the path from QI to error detection in our organization.

METHODS: Sigma analysis of repeated harvesting, wrong demographic data and requested and unrealized analyzes. Use of appropriate statistical tools, namely the T-Test of proportions, using Minitab software.

RESULTS: In 2018 we obtained a mean Sigma of 4,004, 3,632 and 3,627 to QI repeated harvesting, wrong demographic data and requested and unrealized analyzes, respectively. After a critical analysis of the process under review, our organization understand the different aspects of problems and justify any need for additional time and resources. The description of the QI clarifies the meaning of data collected and the actions to be taken to avoid future errors and nonconformities.

CONCLUSIONS: The more frequent errors and non-conformities occur in the preanalytical phase. The development of QI in a laboratory is a fundamental step in providing evidence of quality and in ensuring that continuous improvement activities are undertaken to reduce the risk of errors in clinical practice

Key Words: quality indicators, pre-analytical errors, Sigma levels

\title{
QUALITY INDICATORS TO DETECT PRE-ANALYTICAL ERRORS: USE OF MEDIAN RESULTS AS A PRE-ANALYTICAL INDICATOR
}

\author{
$\underline{\text { R. Ribeiro }}^{1}$, M.J. Sousa ${ }^{1}$, M. Albuquerque ${ }^{1}$, J.G. Sousa ${ }^{1}$, G. Sousa ${ }^{1}$ \\ ${ }^{1}$ Centro de Medicina Laboratorial Dr. Germano de Sousa, Lisboa, Portugal
}

BACKGROUND-AIM: The constant technological development and scientific advances in the new equipment and tests, the type of errors and their frequency in the Clinical Laboratory have been changing. The main reason is the difficulty in controlling the pre-analytical variables, such as harvesting, handling, transporting and sample preparation. The use of quality indicators (QI) has been valued in the management of clinical laboratories to optimize the qualification and quantification of failures in the laboratory processes. In several analytical parameters, the median results value is a robust measure and could be considered as QI according to the specification for the pre-analytical quality. In Centro Medicina Laboratorial Germano de Sousa we used potassium median results as QI in the pre-analytical phase and apply adequate corrective and preventive corrections.

METHODS: The Potassium median results were evaluated, and values have been stratified according to the origin (primary care and hospital laboratory). Use of appropriate statistical tools, namely the Mood Median test, using Minitab software.

RESULTS: The potassium median range was [4.200; 4.700] (95\%IC), and sample origin stratification showed a statistically difference (p-value $<0.001$ ) in potassium medians from different collection points, allow us detected a pre-analytical error in this particular center. After a critical analysis of the process under review, our organization understand the different aspects of problems and justify the need for additional resources and technical training.

CONCLUSIONS: The more frequent errors and non-conformities occur in the pre-analytical phase. The development of QI in a laboratory is a fundamental step in providing evidence of quality and in ensuring that continuous improvement activities are undertaken to reduce the risk of errors in clinical practice. The use of median results of sensitive parameters allows us to detected errors and introduce corrective actions more assertively.

Key Words: quality indicators, pre-analytical errors, median results

\section{THE COST OF POOR BLOOD SPECIMEN QUALITY AND ERRORS IN PREANALYTICAL PROCESSES AT A TERTIARY HOSPITAL SETTING IN R. MACEDONIA}

\author{
$\underline{\text { A. Atanasova Boshku }}^{1}$ \\ ${ }^{1}$ University Clinic of Gynecology and Obstetrics, Medical Faculty, Univ. Snt Cyril and Methody, Skopje, R. Macedonia
}

BACKGROUND-AIM: The complexity of the current health environment has increased the potential for medical errors. Medical errors can occur everywhere in the health system, hospitals, clinics, surgical centers, doctors and the laboratory. The most common causes of laboratory errors are those observed in the pre-analytical phase and affect the clinical and financial outcomes. The most common causes of preanalytical errors are hemolysis, inaccurate patient identification, insufficient sample and clustered samples. Each of these variables has the potential to influence the quality of laboratory tests. The pre-analytical error detected by the laboratory may require re-collection of samples, adding time and time for the processing of the results. The cases in which the error is not timely detected by the laboratory, but is revealed after the result is reported to the physician may adversely affect the diagnosis and / or treatment of the patient.

METHODS: We have made an evaluation of all the wrong samples received in the laboratory for a period 6 months starting from October 2017 to April 2018.

RESULTS: From the total number of samples with identified error in the pre-analytical phase (348, 100\%), the majority of them were coagulated $(226,65 \%)$, with hemolysis $(72,21 \%)$, insufficient volume $(45,13 \%)$ and incorrect patient identification $(5,1 \%)$. A root causes and SWOT 
analysis was performed, to find the most frequent reasons for pre-analytical errors. After defining the weakest phases of the preanalytical process in our hospital setting, we have performed corrective measures.

CONCLUSIONS: Preanalitical errors not only increase redraw cost within the laboratory, but also impact the time and resources required for follow- up or as a result of delays in patient care. The future goals for Macedonia include establishing best practices for blood collection which leads to improving the quality of blood specimens, better public healthcare financing and lowering cost of preanalytical errors.

Key Words: preanalytical errors

\title{
PREANALYTICAL, ANALYTICAL AND POSTANALYTICAL LABORATORY ERRORS: PUBLIC PERCEPTION ANALYZED WITH GOOGLE TRENDS
}

\author{
G.L. Salvagno ${ }^{1}$, G. Lippi ${ }^{1}$ \\ ${ }^{1}$ Section of Clinical Biochemistry, University Hospital of Verona, Verona, Italy
}

BACKGROUND-AIM: Although firm evidence now attest that most laboratory errors emerge from manually intensive activities of the preanalytical phase, anecdotal evidence seemingly suggest that analytical errors are perceived as more frequent. Google Trends (Google Inc. Mountain View, CA, US) has been recently proposed as a reliable tool for estimating the popularity of certain topics, including important healthcare issues, among Internet users. This freely available Web-based software enables analyzing the popularity of specific search queries in Google Search around the world.

METHODS: In order to define the worldwide popularity among Internet users of "preanalytical errors", "analytical errors” and postanalytical errors", an electronic search with no geographical restriction was carried out in Google Trends to identify the number of weekly Google searches (i.e., weekly Google Trends score) for these three keywords between January 2004 and December 2018.

RESULTS: The Google Trends scores (mean and standard deviation) of the three search terms "preanalytical errors", "analytical errors" and postanalytical errors" were $7 \pm 10$ ( $29 \%$ of total Google searches), $17 \pm 12$ ( $71 \%$ of total Google searches) and $0 \pm 0$ ( $0 \%$ of total Google searches). The Google trends scores of analytical errors was significantly higher than that of both preanalytical and postanalytical errors (both $\mathrm{p}<0.001)$, whilst that of preanalytical errors was also higher than that of postanalytical errors $(p<0.001)$. The Google Trends score of both preanalytical $(\mathrm{p}=0.293)$ and analytical $(\mathrm{p}=0.240)$ errors remained identical in the past 5 years compared to the previous period.

CONCLUSIONS: Unlike currently available evidence-based data, the results of this analysis attest that the popularity of analytical errors among Internet users remains more than twice higher than that of preanalytical errors, whilst the general public seems almost unaware of the important burden of postanalytical errors.

Key Words: Google Trends, laboratory errors, preanalytical phase

\section{PATIENT SAFETY AND ASSESSMENT OF PREANALYTIC PROCESS IN CLINICAL LABORATORIES: QUALITY INDICATORS}

\author{
$\underline{\text { d. Iren Emekli }}^{1}$ \\ ${ }^{1}$ ozel izmir hastanesi, Turkey
}

BACKGROUND-AIM: In clinical laboratories total testing process occur from 3 sub-process. Preanalytic process which is the interval between clinical request and beginning of analyze effects test safety on account of patient safety considerably ( $46 \%-68.2 \%)$. The variables that effect preanalytic process must be kept under control. Variables are sources of errors and preanalytic process errors like incorrect request, improperly labeling, insufficient clinical information, hemolyzed samples, improper sample transport, duplicate order, inappropriate container, insufficient sample and damaged sample are errors that cause waste of time and money. Based on these errors quality indicators are determined (IFCC-WG-LEPS). Process performance is evaluated by matching determined quality indicators with target values. In this study, our aim is to evaluate common errors in preanalytical process as quality indicators.

METHODS: Preanalytical process error data between January 2017 and June 2018 were obtained from the laboratory information system. For every type of error monthly percentages have calculated and matched with target levels determined by IFCC-WG-LEPS.

RESULTS: According to quality targets "hemolysed sample” and "clotted sample" had error percentages under "acceptable performance” respectively. Duplicate request, damaged sample, improperly labeling, lipemic samples, sample not received, incorrect sample volume, insufficient sample, improper sample transport, incorrect collection time, inappropriate container, incorrect request, incorrect sample achieved the target. CONCLUSIONS: Our results showed that quality indicators may be useful for evaluation of preanalytical process. According to the quality indicators that not achieved the target, the origin of the errors can be determined and corrective and preventive actions can be carried out. Also monthly trends can be evaluated and precautions can be taken for the prevention from the errors that mostly effect the patient safety (educations, development of instrument, etc.).

Key Words: preanayltical phase 


\title{
INTEGRATED PREANALYTICAL EQA (EQA3) AS PART OF BLOOD GAS SCHEME
}

\author{
L. Pelanti $^{2}$, A. Vanhanen ${ }^{2}$, A. Rauhio ${ }^{1}$, H. Berghäll ${ }^{2}$ \\ ${ }^{1}$ Fimlab Laboratories, Tampere, Finland \\ ${ }^{2}$ Labquality Ltd, Helsinki Finland
}

BACKGROUND-AIM: Labquality's integrated external quality assessment (EQA3) service is a completely new approach to fulfil the requirements of the ISO 15189 standard stating that all steps of the laboratory testing process should be monitored. In addition to the separate preanalytical schemes launched in 2014, Labquality offers several schemes where preanalytical cases have been added to the traditional samples. For the blood gas scheme, EQA3 was introduced in 2017 with preanalytical cases added to two out of four schemes per year.

METHODS: Scenarios from real life are used as case studies in the integrated preanalytical EQA. The results are grouped by the profession of the participants. For this study, we gathered results from all four EQA3 rounds of the blood gas schemes so far. The largest group to report answers were biomedical laboratory scientists (BLS) followed by laboratory assistants, chemists and medical doctors. Finding the expected preanalytical error(s) and the success of determining the expected corrective action defined by the scheme expert was assessed for all cases. RESULTS: $60-75 \%$ of the participants in the schemes responded to the so far voluntary preanalytical question. The number of participants was 2-2,5 times the number of participants in the separate chemistry preanalytical round. $60 \%$ of the largest group (BLS) found the expected action in 2017 and $50 \%$ of all the others grouped together. The success will be compared against the success in 2018.

CONCLUSIONS: According to the results, quite a large portion of the participants would accept samples with clear preanalytical errors and this raises the importance of EQA focus on this step of the laboratory process. Integrated preanalytical EQA is beneficial for the laboratories in that it raises the awareness of the preanalytical factors among all staff members and offers the laboratories a convenient tool for measuring the preanalytical processes.

Key Words: integrated EQA, PT, preanalytical error

\section{AUDIT OVERVIEW OF THE PHLEBOTOMY PRACTICES IN THE NORTH ESTONIA MEDICAL CENTRE}

\author{
$\underline{\text { A. Lellep }}^{1}$, I. Univer ${ }^{1}$, J. Kurm ${ }^{1}$ \\ ${ }^{1}$ Laboratory, North Estonia Medical Centre (NEMC), Tallinn
}

BACKGROUND-AIM: Phlebotomy is the most common procedure and source of preanalytical errors, which go often unnoticed. The aim of our study was to get an overview of the practice in phlebotomy in our hospital, define the current situation and assess the level of compliance with the our hospital phlebotomy guidelines.

METHODS: An audit has been conducted in the NEMC ( 7 clinics, 32 centres, 1,200 beds). Phlebotomy is mostly performed by nurses. There are approximately 2000 nurses in the hospital. Data collection period was from July to December 2017. All observations were performed using a standardized questionnaire (28 questions), which was compiled on the basis of a structured checklist of EFLM WG-PRE (Simundic A-M., et al. 2015). Possible replies were „yes“, „no“ and „not applicable“. Results are presented as counts and percentages.

RESULTS: The audit took place in 15 departments and included observation of 41 phlebotomy procedures carried out by 25 nurses. The error rate for the total phlebotomy procedure was $38.6 \%$ (min 5 errors per nurse and max 17 errors per nurse). Patient identification, disinfection and test tube handling were identified as the key issues. 59\% of the nurses made correct patient identification procedure. $39 \%$ of the nurses sanitized hand appropriately. $46 \%$ of the nurses left the venipuncture site dry. $59 \%$ of the nurses released tourniquet $\leq 60$ s. $37 \%$ of the nurses mixed sample tubes appropriately according to manufacturers specifications.

CONCLUSIONS: The level of skills and compliance with the phlebotomy guidelines was found to be low. This procedure needs immediate attention and improvement. It is necessary to organize mandatory workshops and training courses on phlebotomy procedure issues in the hospital and start mentor programs in the workplaces. Large knowledge base of correct phlebotomy processes is available, but continuous education is a pre-requisite for reliable implementation in daily routine patient care.

Key Words: phlebotomy, guidelines, audit, level of skills

\section{PREANALYTICAL ERRORS IN THE HAEMOSTASIS LABORATORY: CAN WE DO BETTER?}

\author{
$\underline{\text { T. Rodrigues }}{ }^{2}$, Â. Maresch ${ }^{2}$, C. Queiróz ${ }^{2}$, C. Pereira ${ }^{2}$, R. Monteiro ${ }^{1}$, J. Mariano Pego ${ }^{2}$, N. Costa E Silva ${ }^{2}$, F. Rodrigues ${ }^{2}$ \\ ${ }^{1}$ Centro Hospitalar do Baixo Vouga - Clinical Pathology Department, Portugal \\ ${ }^{2}$ Centro Hospitalar e Universitário de Coimbra, Portugal - Clinical Pathology Department, Portugal
}

BACKGROUND-AIM: The Clinical Pathology Department of the Centro Hospitalar e Universitário de Coimbra receives almost 5000 test samples a day, from very different origins like our department collection centre, wards and emergency departments. Despite advanced automation 
in diagnostic labs, there are still considerable errors affecting laboratory results. Most errors occur in the preanalytical phase. Difficulty in achieving standardized procedures for sample collection results in haemostasis laboratory samples being frequently rejected for presenting conditions that may compromise the validity of the tests results.

The objective of this study was to evaluate the leading causes of preanalytical errors that lead to the rejection of haemostasis laboratory samples and assess if an increased awareness for these errors could translate in an inferior occurrence rate.

METHODS: A retrospective analysis of one month of haemostasis tests requests were obtained and the data regarding the number and the causes for sample rejection was analysed, as well as the sample origin. The samples' origin was divided in 2 groups: samples collected in our collection centre, where professionals are expected to be more aware of correct preanalytical procedures, and samples collected in other departments.

RESULTS: 14990 samples were received and 861 of these were rejected (6\%). Rejections were due to insufficient volume (593), clot presence (245), haemolysis (14) and other less frequent reasons. As for the samples' rejection rates, $9 \%$ of the samples that came from outside the lab and $2 \%$ of the ones collected in-house were rejected. The main errors were the same, regardless the origin.

CONCLUSIONS: The overall rejection percentage was 6\%, being the leading cause the collection of a sample with insufficient volume (69\%). An increased awareness and training can be a key factor for the reduction of frequency of such errors.

Key Words: preanalytical errors, coagulation, rejected samples, incorrect volume, awareness

\section{HARMONIZATION OF PRE-ANALYTICAL PROCEDURES AND CROSS-AUDITS WITHIN THE AUSTRIAN BIOBANK NETWORK BBMRI.AT}

$\underline{\text { H. Helmuth }}^{7}$, M. Bayer ${ }^{2}$, F. Heidelinde ${ }^{4}$, M. Gerner ${ }^{7}$, P. Hofer ${ }^{8}$, M. Korb ${ }^{9}$, S. Neururer ${ }^{3}$, T. Perkmann ${ }^{7}$, K. Plattner ${ }^{2}$, K. Sargsyan ${ }^{2}$, E. Steiner ${ }^{5}$, C. Stumptner ${ }^{6}$, I. Walter ${ }^{9}$, M. Wieser ${ }^{9}$, A. Wutte ${ }^{1}$, O. Wagner ${ }^{7}$, K. Zatloukal ${ }^{6}$

${ }^{1} B B M R I-E R I C$, Graz, Austria

${ }^{2}$ Biobank Graz, Medical University of Graz, Graz, Austria

${ }^{3}$ Department of Medical Statistics, Informatics \& Health Economics, Medical University of Innsbruck, Innsbruck, Austria

${ }^{4}$ Department of Obstetrics \& Gynaecology, Medical University of Innsbruck, Innsbruck, Austria

${ }^{5}$ Division of Experimental Urology, Department of Urology, Medical University of Innsbruck, Innsbruck, Austria

${ }^{6}$ Institute of Pathology, Medical University of Graz, Graz, Austria

${ }^{7}$ MedUni Wien Biobank, Department of Laboratory Medicine, Medical University of Vienna, Austria

${ }^{8}$ MedUni Wien Biobank, Department of Pathology, Medical University of Vienna, Vienna, Austria

${ }^{9}$ VetCore Facility for Research, University of Veterinary Medicine Vienna, Vienna, Austria

BACKGROUND-AIM: Non-reproducibility of biomedical research results is a major economic and ethical issue and is frequently produced by poor biomaterial. Standardization of pre-analytical procedures, e.g., in the framework of professional biobank facilities, might help to overcome this problem. All Austrian biobanks related to BBMRI.at (www.bbmri.at) therefore decided to harmonize their pre-analytical procedures by internationally valid documents (CEN/TS on the pre-examination phase) and to mutually examine their quality management (QM) efforts by intra-consortial cross audits.

METHODS: Nine multi-dimensional (secondary material derived from primary material for a particular purpose) CEN/TS were available. CEN/TS were operationalized for biobanking purposes, and process charts were drawn. Self-assessment tools were generated in cooperation with BBMRI-ERIC (www.bbmri-eric.eu). QM trained biobanking experts performed cross-audits according to ISO 9001.

RESULTS: After five years, all BBMRI.at partner biobanks could successfully implement at least one CEN/TS-based pre-analytical workflow. Hence, as of December 2018, 1/4 million samples were labeled by BBMRI-ERIC as CEN/TS compliant after remote audits. Moreover, the first round of intra-consortial QM cross-audits was successfully completed, and results in the form of audit reports were disseminated within the consortium.

CONCLUSIONS: The biobanks of the Austrian biobanking consortium BBMRI.at were highly cooperative in the implementation of common standards. Moreover, they aimed to improve their quality management system by mutual cross-audits, which also increased their collective knowledge. All of these measures might contribute to containing risks to the reproducibility of biomedical results.

Key Words: biobank, BBMRI, pre-analytical phase, technical specifications, harmonization 